Supporting Information

\title{
Diastereo- and Enantioselective Synthesis of Borylated 3- hydroxyoxindoles by Addition of Gem-diborylalkanes to Isatins
}

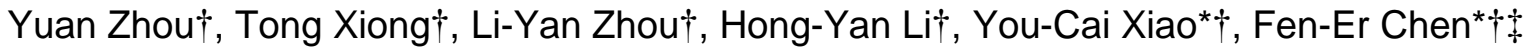

\begin{abstract}
†Key Laboratory of Drug-Targeting and Drug Delivery System of the Ministry of Education and Sichuan Research Center for Drug Precision Industrial Technology, West China School of Pharmacy, Sichuan University, Chengdu 610041, China

Fngineering Center of Catalysis and Synthesis for Chiral Molecules, Department of Chemistry, Fudan University, Shanghai 200433, China
\end{abstract}

E-mail: xiaoliguo1987@scu.edu.cn; rfchen@ fudan.edu.cn 
Table of Contents

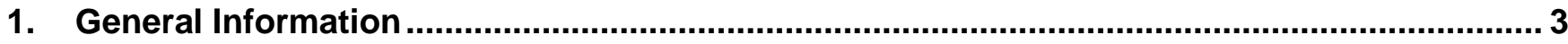

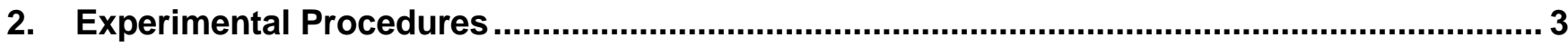

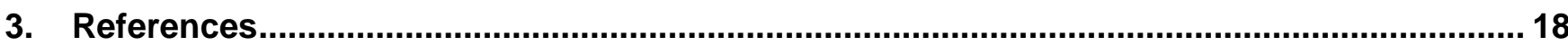

4. Spectral Data

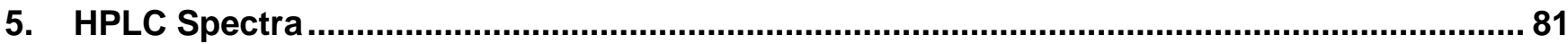

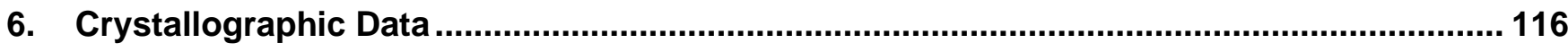




\section{General Information}

All reactions and manipulations which are sensitive to moisture or air were performed under inert atmosphere of argon. Oil bath served as the heat source. All chemicals were purchased from Adamas, Innochem and Aladdin and were used as received. Chromatography was conducted by using 300-400 mesh silica gel. Anhydrous THF was purchased from innochem $(99.9 \%$, extra dry with molecular sieves, water $\leq 50 \mathrm{ppm}$,stabilized with $\mathrm{BHT}$,in resealable bottle). NMR spectra were recorded on a Bruker Ascend spectrometer at $400 \mathrm{MHz}\left({ }^{1} \mathrm{H} \mathrm{NMR}\right), 101 \mathrm{MHz}$ or $151 \mathrm{MHz}\left({ }^{13} \mathrm{C} \mathrm{NMR}\right), 193 \mathrm{MHz}\left({ }^{11} \mathrm{~B} \mathrm{NMR}\right)$, and $376 \mathrm{MHz}\left({ }^{19} \mathrm{~F}\right.$ NMR). NMR spectra were recorded in deuterated chloroform $\left(\mathrm{CDCl}_{3}\right)$ or DMSO- $d 6$ as a solvent, with residual chloroform $\left(\delta 7.26 \mathrm{ppm}\right.$. for ${ }^{1} \mathrm{H}$ NMR and $\delta$ 77.0 ppm. for ${ }^{13} \mathrm{C}$ NMR) or tetramethylsilane (TMS, $\delta 0.00$ ppm. for ${ }^{1} \mathrm{H}$ NMR) or residual DMSO ( $\delta 2.50 \mathrm{ppm}$. for ${ }^{1} \mathrm{H}$ NMR and $\delta 39.5$ ppm. for ${ }^{13} \mathrm{C} N \mathrm{NR}$ ) taken as the inert standard and were reported in ppm. Abbreviations for signal coupling are as follows: $\mathrm{s}=$ singlet, $\mathrm{d}=$ doublet, $\mathrm{t}=$ triplet, $\mathrm{q}=$ quartet, $\mathrm{m}=$ multiplet, $\mathrm{dd}=$ double doublet, and $\mathrm{t}$ = double triplet. Coupling constants were taken from the spectra directly and are uncorrected. Optical rotations were determined using a Rudolph Research Analytical Autopol VI automatic polarimeter. High-resolution mass spectra (HRMS) were recorded on Bruker micrOTOF-Q III, by the ESI method. HPLC analyses were performed using Agilent Technologies 1260 Infinity II with DAICEL Chiralcel AD-H and Chiralpak IC column. Single-Crystal XRay diffraction was recorded at Bruker APEX-II CCD diffractometer.

\section{Experimental Procedures}

\subsection{General synthesis of isatin reagents $(1 a-10)^{1}$}

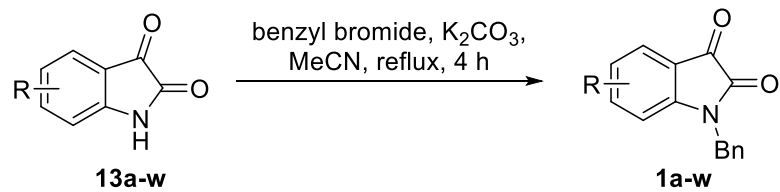

To an MeCN solution $(0.10 \mathrm{M})$ of isatin $\left(1.0\right.$ equiv., $3.0 \mathrm{mmol}$ ) was added $\mathrm{K}_{2} \mathrm{CO}_{3}$ (3.0 equiv., $9.0 \mathrm{mmol}$ ) and benzyl bromide (4.5mmol, 1.50 equiv.) at room temperature. The mixture was heated at reflux for 4 hours. The mixture was cooled, filtered and concentrated in vacuo. The crude reaction mixture was purified by silica gel column chromatography to give the desired benzylation of isatin derivatives. The products matched the known ${ }^{1} \mathrm{H}$ NMR spectra.

\subsection{General synthesis of isatin reagents $(1 \mathrm{u}-1 \mathrm{w})^{2}$}

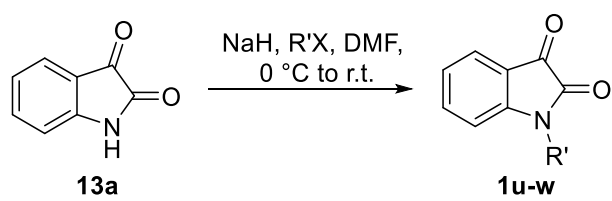

13a (1.0 equiv., $3.0 \mathrm{mmol}$,) was dissolved in anhydrous DMF (15 mL), and the resultant solution was cooled to $0{ }^{\circ} \mathrm{C}$, whereupon sodium hydride ( $60 \%$ dispersion in mineral oil, 1.2 equiv., $3.6 \mathrm{mmol}$ ) was added in one portion and stirred for 5-10 minutes. Alkyl or aryl halide (1.1 equiv., $3.3 \mathrm{mmol}$ ) was added and the reaction was stirred at $0{ }^{\circ} \mathrm{C}$ for 30 minutes, and the reaction was allowed to warm up to room temperature. The reaction was monitored by TLC until 13a was fully consumed. The reaction mixture was then poured into saturated aqueous $\mathrm{NH}_{4} \mathrm{Cl}$ and extracted with ethyl acetate. The combined organic portions were washed with water and brine, dried $\left(\mathrm{Na}_{2} \mathrm{SO}_{4}\right)$, filtered, and concentrated. The crude reaction mixture was purified by silica gel column chromatography to give $\mathrm{N}$-substituted isatins. The products matched the known ${ }^{1} \mathrm{H}$ NMR spectra. 


\subsection{General synthesis of substituted diboryl reagents $(5 \mathrm{a}-5 \mathrm{~g})^{3}$}

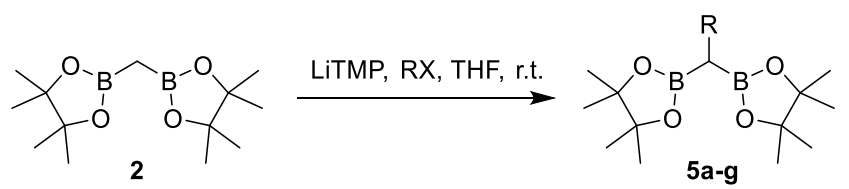

A $50 \mathrm{~mL}$ oven-dried Schlenk flask equipped with a magnetic stir bar and a septum was evacuated and backfilled with argon three times. TMPH (2,2,6,6-tetramethylpiperidine, $0.7 \mathrm{~mL}, 4.0 \mathrm{mmol})$ was added via syringe, followed by anhydrous THF to give approximately $15.0 \mathrm{~mL}$ of solution. The mixture was cooled to $-78{ }^{\circ} \mathrm{C}$ and stirred for 10 minutes. $n$-BuLi $(2.5 \mathrm{M}$ in hexanes, $1.6 \mathrm{~mL}$ $4.0 \mathrm{mmol}$ ) was added dropwise and reaction mixture was stirred for 30 minutes at $-78{ }^{\circ} \mathrm{C}$, then warmed to room temperature $\left(25^{\circ} \mathrm{C}\right)$ and stirred for 4 hours.

Diboryl methane $2(1.00 \mathrm{~g}, 3.7 \mathrm{mmol})$ dissolved in $10.0 \mathrm{~mL}$ THF in a Schlenk tube under an atmosphere of argon. Both flasks were allowed to cool to $0{ }^{\circ} \mathrm{C}$. The LiTMP solution was then cannula transferred to the diboryl methane flask with stirring. After the transfer, the reaction was allowed to stir for $10 \mathrm{~min}$ at $0{ }^{\circ} \mathrm{C}$. Alkyl halide $(9.0 \mathrm{mmol})$ was then added to the reaction via a syringe and the reaction was allowed to warm up to $25{ }^{\circ} \mathrm{C}$ over 12 hours with stirring. The reaction was quenched with $50 \mathrm{~mL}$ of saturated aqueous solution of $\mathrm{NH}_{4} \mathrm{Cl}$. The biphasic'c mixture was extracted 3 times with diethyl ether $(10 \mathrm{~mL} \times 3)$, and the combined organic extracts were dried over $\mathrm{Na}_{2} \mathrm{SO}_{4}$, filtered, and concentrated in vacuo. The crude reaction mixture was purified by silica gel column chromatography to give the desired diboryl reagent. The products matched the known ${ }^{1} \mathrm{H}$ NMR spectra.

\section{2,2'-(ethane-1,1-diyl)bis(4,4,5,5-tetramethyl-1,3,2-dioxaborolane) (5a)}<smiles>CC(B1OC(C)(C)C(C)(C)O1)B1OC(C)(C)C(C)(C)O1</smiles>
Prepared by the general procedure from iodomethane, and $\mathbf{5 a}$ was isolated as a colorless oil. $(0.81 \mathrm{~g}$, $76.7 \%$ yield), eluent (petroleum ether/ethyl acetate $=30: 1$ ).

${ }^{1} \mathrm{H}$ NMR $(400 \mathrm{MHz}$, Chloroform-d) $\delta 1.23(\mathrm{~d}, J=3.2 \mathrm{~Hz}, 24 \mathrm{H}), 1.04(\mathrm{~d}, J=7.3 \mathrm{~Hz}, 3 \mathrm{H}), 0.76-0.70(\mathrm{~m}$, $1 \mathrm{H})$.

\section{2,2'-(butane-1,1-diyl)bis(4,4,5,5-tetramethyl-1,3,2-dioxaborolane) (5b)}<smiles>CCCC(B1OC(C)(C)C(C)(C)O1)B1OC(C)(C)C(C)(C)O1</smiles>
Prepared by the general procedure from 3-bromopropane, and $\mathbf{5 b}$ was isolated as a colorless oil. (1.7 $\mathrm{g}$, $88.5 \%$ yield), eluent (petroleum ether/ethyl acetate $=30: 1$ ).

${ }^{1} \mathrm{H}$ NMR (400 MHz, Chloroform-d) $\delta 1.56-1.49(\mathrm{~m}, 2 \mathrm{H}), 1.34-1.26(\mathrm{~m}, 2 \mathrm{H}), 1.23(\mathrm{~d}, J=3.3 \mathrm{~Hz}, 24 \mathrm{H})$, $0.87(\mathrm{t}, J=7.3 \mathrm{~Hz}, 3 \mathrm{H}), 0.74(\mathrm{t}, J=7.9 \mathrm{~Hz}, 1 \mathrm{H})$.

\section{2,2'-(3-phenylpropane-1,1-diyl)bis(4,4,5,5-tetramethyl-1,3,2-dioxaborolane) (5c)}<smiles>CC1(C)OB(C(CCc2ccccc2)B2OC(C)(C)C(C)(C)O2)OC1(C)C</smiles>

Prepared by the general procedure from (2-bromoethyl)benzene, and $\mathbf{5 c}$ was isolated as a colorless oil. (0.88 g, 65.4\% yield), eluent (petroleum ether/ethyl acetate $=30: 1)$.

${ }^{1} \mathrm{H}$ NMR $(400 \mathrm{MHz}$, Chloroform- $d) \delta 7.25-7.21(\mathrm{~m}, 2 \mathrm{H}), 7.19-7.11(\mathrm{~m}, 3 \mathrm{H}), 2.61-2.56(\mathrm{~m}, 2 \mathrm{H}), 1.90$ $-1.79(\mathrm{~m}, 2 \mathrm{H}), 1.23(\mathrm{~d}, J=3.4 \mathrm{~Hz}, 24 \mathrm{H}), 0.81(\mathrm{t}, J=7.9 \mathrm{~Hz}, 1 \mathrm{H})$.

\section{2,2'-(but-3-ene-1,1-diyl)bis(4,4,5,5-tetramethyl-1,3,2-dioxaborolane) (5d)}<smiles>C=CCC(B1OC(C)(C)C(C)(C)O1)B1OC(C)(C)C(C)(C)O1</smiles>

Prepared by the general procedure from allyl bromide, and $\mathbf{5 d}$ was isolated as a colorless oil. $(0.86 \mathrm{~g}$, $75.5 \%$ yield), eluent (petroleum ether/ethyl acetate $=30: 1$ ) 
${ }^{1} \mathrm{H}$ NMR $(400 \mathrm{MHz}$, Chloroform-d) $\delta 5.92-5.83(\mathrm{~m}, 1 \mathrm{H}), 5.02-5.00(\mathrm{~m}, 1 \mathrm{H}), 4.88-4.87(\mathrm{~m}, 1 \mathrm{H}), 4.86-4.85(\mathrm{~m}, 1 \mathrm{H}), 2.32-2.27$ $(\mathrm{m}, 2 \mathrm{H}), 1.22(\mathrm{~d}, J=4.6 \mathrm{~Hz}, 24 \mathrm{H}), 0.85(\mathrm{t}, J=8.0 \mathrm{~Hz}, 1 \mathrm{H})$.

\section{2,2'-(4-methylpent-3-ene-1,1-diyl)bis(4,4,5,5-tetramethyl-1,3,2-dioxaborolane) (5e)}<smiles>CC(C)=CCC(B1OC(C)(C)C(C)(C)O1)B1OC(C)(C)C(C)(C)O1</smiles>

Prepared by the general procedure from 3,3-dimethylallyl bromide, and $\mathbf{5 e}$ was isolated as a colorless oil. (0.92 $\mathrm{g}, 72.4 \%$ yield), eluent (petroleum ether/ethyl acetate $=30: 1)$.

${ }^{1}$ H NMR $(400 \mathrm{MHz}$, Chloroform-d) $\delta 5.12-5.07(\mathrm{~m}, 1 \mathrm{H}), 2.21(\mathrm{t}, J=7.7 \mathrm{~Hz}, 2 \mathrm{H}), 1.62(\mathrm{~d}, J=9.1 \mathrm{~Hz}$, $6 \mathrm{H}), 1.22(\mathrm{~d}, J=4.7 \mathrm{~Hz}, 24 \mathrm{H}), 0.76(\mathrm{t}, J=8.1 \mathrm{~Hz}, 1 \mathrm{H})$.

\section{(3,3-bis(4,4,5,5-tetramethyl-1,3,2-dioxaborolan-2-yl)propoxy)(tert-butyl)dimethylsilane (5f)}<smiles>CC1(C)OB(C(CCO[SbH3])B2OC(C)(C)C(C)(C)O2)OC1(C)C</smiles>

Prepared by the general procedure from (2-bromoethoxy)-tert-butyldimethylsilane, and $\mathbf{5} \mathbf{f}$ was isolated as a colorless oil. $(1.23 \mathrm{~g}, 78.0 \%$ yield), eluent (petroleum ether/ethyl acetate $=30: 1)$.

${ }^{1} \mathbf{H}$ NMR (400 MHz, Chloroform-d) $\delta 3.54(\mathrm{t}, J=7.2 \mathrm{~Hz}, 2 \mathrm{H}), 1.81-1.74(\mathrm{~m}, 2 \mathrm{H}), 1.21(\mathrm{~d}, J=4.1 \mathrm{~Hz}$, $24 \mathrm{H}), 0.88(\mathrm{~s}, 9 \mathrm{H}), 0.78(\mathrm{t}, J=7.7 \mathrm{~Hz}, 1 \mathrm{H}), 0.04(\mathrm{~s}, 6 \mathrm{H})$.

\section{2,2'-(2-phenylethane-1,1-diyl)bis(4,4,5,5-tetramethyl-1,3,2-dioxaborolane) (5g)}<smiles>CC1(C)OB(C(Cc2ccccc2)B2OC(C)(C)C(C)(C)O2)OC1(C)C</smiles>

Prepared by the general procedure from benzyl bromide, and $\mathbf{5 g}$ was isolated as a colorless oil. (1.10 $\mathbf{g}$, $83.1 \%$ yield), eluent (petroleum ether/ethyl acetate $=30: 1$ ).

${ }^{1} \mathrm{H}$ NMR $(400 \mathrm{MHz}$, Chloroform- $d) \delta 7.25-7.18(\mathrm{~m}, 4 \mathrm{H}), 7.14-7.07(\mathrm{~m}, 1 \mathrm{H}), 2.88(\mathrm{~d}, \mathrm{~J}=8.3 \mathrm{~Hz}, 2 \mathrm{H})$, $1.17(\mathrm{~d}, J=4.3 \mathrm{~Hz}, 25 \mathrm{H})$.

\subsection{General procedure A: Cu-catalyzed 1,2-addition reaction of diboryl methane}<smiles></smiles>

$1 a-w$
(1) $\mathrm{Cu}\left(\mathrm{CH}_{3} \mathrm{CN}\right)_{4} \mathrm{PF}_{6}(10 \mathrm{~mol} \%)$, L1 (20 mol\%), LiOtBu (2.5 equiv.),<smiles></smiles>
2

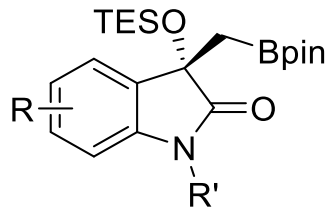

4a-w

A mixture of $\mathrm{Cu}\left(\mathrm{CH}_{3} \mathrm{CN}\right)_{4} \mathrm{PF}_{6}(10 \mathrm{~mol} \%, 0.01 \mathrm{mmol})$, (S)-Monophos $\mathrm{L} 1$ (20 mol \%, $\left.0.02 \mathrm{mmol}\right)$, and LiOtBu (0.5 equiv, 0.05 $\mathrm{mmol})$ in THF $(0.5 \mathrm{~mL})$ was stirred for 45 minutes at $25{ }^{\circ} \mathrm{C}$ in a Schlenk tube under an atmosphere of argon. Diborylmethane 2 (1.5 equiv., $0.15 \mathrm{mmol}$ ) and LiO'Bu (2.0 equiv., $0.2 \mathrm{mmol}$ ) were weighed into a separate vial and dissolved in THF (1.0 mL) under an atmosphere of argon. An aliquot of the catalyst solution $(0.5 \mathrm{~mL})$ and stir bar was then transferred to the vial containing the diborylmethane and LiO'Bu. After 15 minutes at $25^{\circ} \mathrm{C}$, the substituted $\mathbf{1 a - 1 w}$ (1.0 equiv, $0.1 \mathrm{mmol}$ in $0.5 \mathrm{~mL}$ THF) was added to the vial via syringe. The reaction was kept at $60{ }^{\circ} \mathrm{C}$ for 2.5 hours and agitated periodically. The reaction was quenched with saturated aqueous solution of $\mathrm{NH}_{4} \mathrm{Cl}(1.5 \mathrm{~mL})$, and the aqueous layer extracted three times with ethyl acetate $(2 \mathrm{~mL} \times 3)$. The combined organic extracts were dried over $\mathrm{Na}_{2} \mathrm{SO}_{4}$, filtered, and concentrated in vacuo. The crude reaction mixture was then taken on the next step without purification. 
Add imidazole (1.0 equiv, $0.1 \mathrm{mmol}$ ), DMAP (1.0 equiv, $0.1 \mathrm{mmol})$ and TESCl $(2.0$ equiv, $0.2 \mathrm{mmol})$ to a solution of the crude hydroxyboronate in $\mathrm{DCM}(2.0 \mathrm{~mL})$. Stir the reaction mixture for overnight. The reaction was quenched with saturated aqueous solution of $\mathrm{NaHCO}_{3}(4.0 \mathrm{~mL})$, and the aqueous layer extracted three times with $\mathrm{DCM}(5 \mathrm{~mL} \times 3)$. The combined organic extracts were dried over $\mathrm{Na}_{2} \mathrm{SO}_{4}$, filtered, and concentrated in vacuo. The product was purified by column chromatography on silica gel.

\subsection{General procedure B: Cu-catalyzed 1,2-addition reaction of secondary alkyl boronates}<smiles>O=C1C(=O)N(Cc2ccccc2)c2ccccc21</smiles>

$1 \mathrm{a}$<smiles>[R]C([R8]Br)[B][Ba]</smiles>

$5 a-g$
(1) $\mathrm{Cu}\left(\mathrm{CH}_{3} \mathrm{CN}\right)_{4} \mathrm{PF}_{6}(10 \mathrm{~mol} \%)$, L1 (20 mol\%), LiO'Bu (2.5 equiv.), $\mathrm{THF}, 60^{\circ} \mathrm{C}, 8 \mathrm{~h}$

(2) imidazole, DMAP, TESCI, DCM, r.t., $12 \mathrm{~h}$<smiles>[R]C([R8]#N)[C@]1(OC#N)C(=O)N(Cc2ccccc2)c2ccccc21</smiles>

$6 a-g$

A mixture of $\mathrm{Cu}\left(\mathrm{CH}_{3} \mathrm{CN}\right)_{4} \mathrm{PF}_{6}(10 \mathrm{~mol} \%, 0.01 \mathrm{mmol})$, (S)-Monophos $\mathbf{L 1}$ (20 mol \%, $\left.0.02 \mathrm{mmol}\right)$, and LiOtBu (0.5 equiv, 0.05 $\mathrm{mmol})$ in THF $(0.5 \mathrm{~mL})$ was stirred for 45 minutes at $25{ }^{\circ} \mathrm{C}$ in a Schlenk tube under an atmosphere of argon. Secondary alkyl boronates 5a-g (1.5 equiv., $0.15 \mathrm{mmol}$ ) and LiO'Bu (2.0 equiv., $0.2 \mathrm{mmol}$ ) were weighed into a separate vial and dissolved in THF $(1.0 \mathrm{~mL})$ under an atmosphere of argon. An aliquot of the catalyst solution $(0.5 \mathrm{~mL})$ and stir bar was then transferred to the vial containing the diborylmethane and LiO'Bu. After 15 minutes at $25^{\circ} \mathrm{C}, 1 \mathrm{a}(1.0$ equiv, $0.1 \mathrm{mmol}$ in $0.5 \mathrm{~mL}$ THF) was added to the vial via syringe. The reaction was kept at $60^{\circ} \mathrm{C}$ for 8 hours and agitated periodically. The reaction was quenched with saturated aqueous solution of $\mathrm{NH}_{4} \mathrm{Cl}(1.5 \mathrm{~mL})$, and the aqueous layer extracted three times with ethyl acetate $(2 \mathrm{~mL} \times 3)$. The combined organic extracts were dried over $\mathrm{Na}_{2} \mathrm{SO}_{4}$, filtered, and concentrated in vacuo. The crude reaction mixture was then taken on the next step without purification.

Add imidazole (1.0 equiv, $0.1 \mathrm{mmol}$ ), DMAP (1.0 equiv, $0.1 \mathrm{mmol}$ ) and TESCl (2.0 equiv, $0.2 \mathrm{mmol}$ ) to a solution of the crude hydroxyboronate in DCM $(2.0 \mathrm{~mL})$. Stir the reaction mixture for overnight. The reaction was quenched with saturated aqueous solution of $\mathrm{NaHCO}_{3}(4.0 \mathrm{~mL})$, and the aqueous layer extracted three times with $\mathrm{DCM}(5 \mathrm{~mL} \times 3)$. The combined organic extracts were dried over $\mathrm{Na}_{2} \mathrm{SO}_{4}$, filtered, and concentrated in vacuo. The product was purified by column chromatography on silica gel.

\section{(S)-1-benzyl-3-((4,4,5,5-tetramethyl-1,3,2-dioxaborolan-2-yl)methyl)-3-((triethylsilyl)oxy)indolin-2-one (4a)}<smiles>CO[C@@]1(CB2OC(C)(C)C(C)(C)O2)C(=O)N(C)c2ccccc21</smiles>

$\mathrm{Bn}$

Prepared by the general procedure A, and $\mathbf{4 a}$ was isolated as a colorless oil, $93 \%$ ee. $(32.6 \mathrm{mg}, 66 \%$ yield), eluent (petroleum ether/ethyl acetate $=30: 1) .{ }^{1} \mathrm{H}$ NMR $(400 \mathrm{MHz}, \mathrm{DMSO}-d 6) \delta 7.45-7.39(\mathrm{~m}$ $3 \mathrm{H}), 7.36-7.21(\mathrm{~m}, 4 \mathrm{H}), 7.02(\mathrm{t}, J=7.5 \mathrm{~Hz}, 1 \mathrm{H}), 6.91(\mathrm{~d}, J=7.8 \mathrm{~Hz}, 1 \mathrm{H}), 4.88-4.78(\mathrm{~m}, 2 \mathrm{H}), 1.64-$ $1.46(\mathrm{~m}, 2 \mathrm{H}), 0.95(\mathrm{~s}, 6 \mathrm{H}), 0.85(\mathrm{~s}, 6 \mathrm{H}), 0.75(\mathrm{t}, J=7.9 \mathrm{~Hz}, 9 \mathrm{H}), 0.40-0.25(\mathrm{~m}, 6 \mathrm{H}) .{ }^{13} \mathrm{C}$ NMR $(101$ $\mathrm{MHz}$, DMSO-d6) $\delta 177.1,142.7,136.7,132.2,129.8,128.9,128.3,127.9,124.3,122.7,109.6,83.2$, 76.1, 43.7, 24.8, 24.5, 7.0, 5.6. ${ }^{11} \mathrm{~B}$ NMR (193 MHz, Chloroform-d) $\delta 32.74$. HRMS (ESI) m/z calcd for $\mathrm{C}_{28} \mathrm{H}_{40} \mathrm{BNNaO}_{4} \mathrm{Si}[\mathrm{M}+\mathrm{Na}]^{+}$: 516.2717, found: 516.2720. [a $]_{D}{ }^{25}=-8.5(c=0.21$, DCM). HPLC $(C H I R A L C E L ~ A D-H), n$-hexane/2-propanol $=95 / 5$, flow rate 0.8 $\mathrm{mL} / \mathrm{min}$, detection at $254 \mathrm{~nm}, t_{\mathrm{R}}=8.1 \mathrm{~min}$ (major), $16.4 \mathrm{~min}$ (minor).

(S)-1-benzyl-5-methyl-3-((4,4,5,5-tetramethyl-1,3,2-dioxaborolan-2-yl)methyl)-3-((triethylsilyl)oxy)indolin-2one (4b)<smiles>Cc1ccc2c(c1)[C@](CB1OC(C)(C)C(C)(C)O1)(OC(F)(F)F)C(=O)N2Cc1ccccc1</smiles>

Prepared by the general procedure A, and $\mathbf{4 b}$ was isolated as a colorless oil, $96 \%$ ee. (34.5 mg $68 \%$ yield), eluent (petroleum ether/ethyl acetate $=30: 1) .{ }^{1} \mathrm{H}$ NMR $(400 \mathrm{MHz}$, Chloroform- $d) \delta 7.40$ - $7.38(\mathrm{~m}, 2 \mathrm{H}), 7.32-7.25(\mathrm{~m}, 4 \mathrm{H}), 6.93(\mathrm{~d}, J=7.9 \mathrm{~Hz}, 1 \mathrm{H}), 6.54(\mathrm{~d}, J=7.9 \mathrm{~Hz}, 1 \mathrm{H}), 4.89-4.75$ (m, 2H), $2.28(\mathrm{~s}, 3 \mathrm{H}), 1.78-1.66(\mathrm{~m}, 2 \mathrm{H}), 1.01(\mathrm{~s}, 6 \mathrm{H}), 0.91(\mathrm{~s}, 6 \mathrm{H}), 0.84(\mathrm{t}, J=7.9 \mathrm{~Hz}, 9 \mathrm{H}), 0.60-$ $0.38(\mathrm{~m}, 6 \mathrm{H}) .{ }^{13} \mathrm{C}$ NMR $(151 \mathrm{MHz}$, Chloroform-d) $\delta 177.7,140.2,136.2,132.8,131.8,129.1$, 
128.6, 127.8, 127.4, 124.9, 108.6, 83.1, 76.2, 44.3, 24.6, 24.4, 21.0, 6.8, 5.8. ${ }^{11}$ B NMR (193 MHz, Chloroform-d) $\delta 32.12 . ~ H R M S$ (ESI) $\mathrm{m} / \mathrm{z}$ calcd for $\mathrm{C}_{29} \mathrm{H}_{42} \mathrm{BNNaO}_{4} \mathrm{Si}[\mathrm{M}+\mathrm{Na}]^{+}: 530.2874$, found: 530.2877 . [ $\left.\alpha\right]_{\mathrm{D}}{ }^{25}=-38.33(c=0.11$, DCM). HPLC (CHIRALCEL AD$\mathrm{H}$ ), $n$-hexane/2-propanol $=95 / 5$, flow rate $0.8 \mathrm{~mL} / \mathrm{min}$, detection at $254 \mathrm{~nm}, t_{\mathrm{R}}=7.5 \mathrm{~min}$ (major), $11.4 \mathrm{~min}$ (minor).

\section{(S)-1-benzyl-5-methoxy-3-((4,4,5,5-tetramethyl-1,3,2-dioxaborolan-2-yl)methyl)-3-((triethylsilyl)oxy)indolin-}

\section{2-one (4c)}

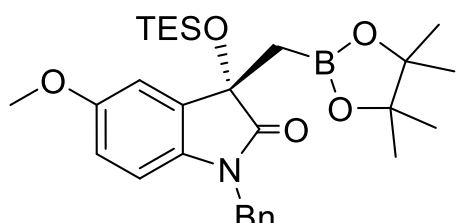

Prepared by the general procedure A, and $\mathbf{4 c}$ was isolated as a colorless oil, $93 \%$ ee. $(32.4 \mathrm{mg}$, $62 \%$ yield), eluent (petroleum ether/ethyl acetate $=30: 1) .{ }^{1} \mathrm{H}$ NMR $(400 \mathrm{MHz}$, DMSO-d6) $\delta 7.42$ $-7.40(\mathrm{~m}, 2 \mathrm{H}), 7.35-7.32(\mathrm{~m}, 2 \mathrm{H}), 7.29-7.25(\mathrm{~m}, 1 \mathrm{H}), 7.12(\mathrm{~s}, 1 \mathrm{H}), 6.81(\mathrm{~s}, 2 \mathrm{H}), 4.86-4.74$ (m, 2H), $3.70(\mathrm{~s}, 3 \mathrm{H}), 1.63(\mathrm{~d}, J=14.5 \mathrm{~Hz}, 1 \mathrm{H}), 1.37(\mathrm{~d}, J=14.5 \mathrm{~Hz}, 1 \mathrm{H}), 1.02(\mathrm{~s}, 6 \mathrm{H}), 0.94$ (s $6 \mathrm{H}), 0.77(\mathrm{t}, J=7.9 \mathrm{~Hz}, 9 \mathrm{H}), 0.41-0.25(\mathrm{~m}, 6 \mathrm{H}) .{ }^{13} \mathrm{C}$ NMR $(151 \mathrm{MHz}, \mathrm{DMSO}-\mathrm{d} 6) \delta 177.0$, 155.8, 136.7, 135.8, 133.4, 128.9, 128.2, 127.8, 114.2, 111.6, 110.1, 83.2, 76.4, 56.0, 43.7, 24.8, 24.7, 7.0, 5.6. ${ }^{11} \mathrm{~B}$ NMR (193 MHz DMSO-d6) $\delta$ 32.04. HRMS (ESI) $\mathrm{m} / \mathrm{z}$ calcd for $\mathrm{C}_{29} \mathrm{H}_{42} \mathrm{BNNaO}_{5} \mathrm{Si}[\mathrm{M}+\mathrm{Na}]^{+}: 546.2823$, found: 546.2828 . $[\alpha]_{\mathrm{D}}{ }^{25}=+4.67(c=0.10$ DCM). HPLC (CHIRALCEL AD-H), $n$-hexane/2-propanol $=95 / 5$, flow rate $0.8 \mathrm{~mL} / \mathrm{min}$, detection at $254 \mathrm{~nm}, t_{\mathrm{R}}=10.5 \mathrm{~min}(\mathrm{major})$ $21.8 \min$ (minor).

\section{(S)-1-benzyl-5-isopropyl-3-((4,4,5,5-tetramethyl-1,3,2-dioxaborolan-2-yl)methyl)-3-((triethylsilyl)oxy) indolin-2-one (4d)}<smiles>CC(C)c1ccc2c(c1)[C@](CB1OC(C)(C)C(C)(C)O1)(OC(F)(F)F)C(=O)N2</smiles>

$\mathrm{Bn}$

Prepared by the general procedure A, and $\mathbf{4 d}$ was isolated as a colorless oil, $94 \%$ ee. $(27.3 \mathrm{mg}$, $51 \%$ yield), eluent (petroleum ether/ethyl acetate $=30: 1) .{ }^{1} \mathrm{H}$ NMR $(400 \mathrm{MHz}$, Chloroform- $d) \delta$ $7.42-7.40(\mathrm{~m}, 2 \mathrm{H}), 7.36-7.23(\mathrm{~m}, 4 \mathrm{H}), 6.98(\mathrm{dd}, J=8.1,1.9 \mathrm{~Hz}, 1 \mathrm{H}), 6.58(\mathrm{~d}, J=8.0 \mathrm{~Hz}, 1 \mathrm{H})$, $4.92(\mathrm{~d}, J=15.5 \mathrm{~Hz}, 1 \mathrm{H}), 4.74(\mathrm{~d}, J=15.5 \mathrm{~Hz}, 1 \mathrm{H}), 2.88-2.81(\mathrm{~m}, 1 \mathrm{H}), 1.80(\mathrm{~d}, J=14.8 \mathrm{~Hz}$ $1 \mathrm{H}), 1.67(\mathrm{~d}, J=14.8 \mathrm{~Hz}, 1 \mathrm{H}), 1.20(\mathrm{~d}, J=6.9 \mathrm{~Hz}, 6 \mathrm{H}), 1.02(\mathrm{~s}, 6 \mathrm{H}), 0.90(\mathrm{~s}, 6 \mathrm{H}), 0.83$ (t, $J=$ $7.9 \mathrm{~Hz}, 9 \mathrm{H}), 0.41(\mathrm{~m}, 6 \mathrm{H}) .{ }^{13} \mathrm{C}$ NMR $(101 \mathrm{MHz}$, Chloroform-d) $\delta 177.8,143.1,140.5,136.2,132.5,128.6,127.9,127.4,126.8,122.4$ 108.6, 83.0, 76.2, 44.4, 33.9, 24.6, 24.4, 24.0, 6.8, 5.9. ${ }^{11}$ B NMR (193 MHz, Chloroform-d) $\delta 32.43$. HRMS (ESI) m/z calcd for $\mathrm{C}_{31} \mathrm{H}_{46} \mathrm{BNNaO}_{4} \mathrm{Si}[\mathrm{M}+\mathrm{Na}]^{+}: 558.3187$, found: 558.3191. [a $]_{D}{ }^{25}=+1.67$ (c = 0.16, DCM). HPLC (CHIRALCEL AD-H), $n$-hexane/2propanol $=95 / 5$, flow rate $0.8 \mathrm{~mL} / \mathrm{min}$, detectionn at $254 \mathrm{~nm}, t_{\mathrm{R}}=5.9 \mathrm{~min}$ (major), $7.2 \mathrm{~min}$ (minor).

\section{(S)-1-benzyl-3-((4,4,5,5-tetramethyl-1,3,2-dioxaborolan-2-yl)methyl)-3-((triethylsilyl)oxy)-5-(trifluoro} methoxy)indolin-2-one (4e)

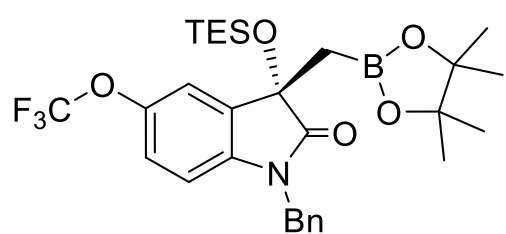

Prepared by the general procedure $\mathrm{A}$, and $\mathbf{4 e}$ was isolated as a colorless oil, $96 \%$ ee. $(26.0$ $\mathrm{mg}, 45 \%$ yield), eluent (petroleum ether/ethyl acetate $=30: 1) .{ }^{1} \mathbf{H}$ NMR $(400 \mathrm{MHz}$, DMSOd6) $\delta 7.60(\mathrm{~d}, J=2.5 \mathrm{~Hz}, 1 \mathrm{H}), 7.43-7.41(\mathrm{~m}, 2 \mathrm{H}), 7.36-7.32(\mathrm{~m}, 2 \mathrm{H}), 7.30-7.26(\mathrm{~m}, 2 \mathrm{H})$ $7.05(\mathrm{~d}, J=8.5 \mathrm{~Hz}, 1 \mathrm{H}), 4.91-4.81(\mathrm{~m}, 2 \mathrm{H}), 1.48(\mathrm{~d}, J=14.6 \mathrm{~Hz}, 1 \mathrm{H}), 1.41(\mathrm{~d}, J=14.7 \mathrm{~Hz}$, $1 \mathrm{H}), 1.00(\mathrm{~s}, 6 \mathrm{H}), 0.91(\mathrm{~s}, 6 \mathrm{H}), 0.74(\mathrm{t}, \mathrm{J}=7.9 \mathrm{~Hz}, 9 \mathrm{H}), 0.24-0.37(\mathrm{~m}, 6 \mathrm{H}) .{ }^{13} \mathrm{C}$ NMR $(101$ MHz, DMSO-d6) $\delta 176.7,143.7$ (d, $J=2.3 \mathrm{~Hz}$ ), 141.2, 135.8, 133.4, 128.5, 127.9, 127.6, 122.6, 120.2 (q, $J=254.0 \mathrm{~Hz}), 117.9$ 110.2, 82.9, 75.6, 43.3, 24.3, 24.0, 6.4, 5.1. ${ }^{11} \mathrm{~B}$ NMR (193 MHz, Chloroform-d) $\delta 33.57 .{ }^{19} \mathrm{~F}$ NMR (376 MHz, Chloroform- $\left.d\right) \delta-58.34$ HRMS (ESI) $\mathrm{m} / \mathrm{z}$ calcd for $\mathrm{C}_{29} \mathrm{H}_{39} \mathrm{BF}_{3} \mathrm{NnaO}_{5} \mathrm{Si}[\mathrm{M}+\mathrm{Na}]^{+}: 600.2540$, found: 600.2541 . $[\alpha]_{\mathrm{D}}{ }^{25}=-4.17$ (c $=0.11$, DCM). HPLC $\left(\right.$ CHIRALCEL AD-H), $n$-hexane/2-propanol = 95/5, flow rate $0.8 \mathrm{~mL} / \mathrm{min}$, detection at $254 \mathrm{~nm}, t_{\mathrm{R}}=5.2 \mathrm{~min}$ (major), $6.2 \mathrm{~min}$ (minor)

\section{(S)-1-benzyl-5-fluoro-3-((4,4,5,5-tetramethyl-1,3,2-dioxaborolan-2-yl)methyl)-3-((triethylsilyl)oxy)indolin-2-} one (4f)<smiles>CC1(C)OB(C[C@]2(OC(F)(F)F)C(=O)N(Cc3ccccc3)c3ccc(F)cc32)OC1(C)C</smiles>

Prepared by the general procedure $\mathrm{A}$, and $\mathbf{4 f}$ was isolated as a colorless oil, $90 \%$ ee. $(29.1 \mathrm{mg}$ $57 \%$ yield), eluent (petroleum ether/ethyl acetate $=30: 1) .{ }^{1} \mathrm{H}$ NMR $(400 \mathrm{MHz}$, DMSO-d6) $\delta 7.44$ $7.42(\mathrm{~m}, 2 \mathrm{H}), 7.36-7.26(\mathrm{~m}, 4 \mathrm{H}), 7.11-7.09(\mathrm{~m}, 1 \mathrm{H}), 6.96-6.93(\mathrm{~m}, 1 \mathrm{H}), 4.88-4.79(\mathrm{~m}, 2 \mathrm{H})$ $1.65(\mathrm{~d}, J=14.5 \mathrm{~Hz}, 1 \mathrm{H}), 1.44(\mathrm{~d}, J=14.5 \mathrm{~Hz}, 1 \mathrm{H}), 1.00(\mathrm{~s}, 6 \mathrm{H}), 0.92(\mathrm{~s}, 6 \mathrm{H}), 0.76(\mathrm{t}, J=7.9 \mathrm{~Hz}$ $9 \mathrm{H}), 0.42-0.26(\mathrm{~m}, 6 \mathrm{H}) .{ }^{13} \mathrm{C}$ NMR $(101 \mathrm{MHz}$, DMSO-d6) $\delta 176.5,158.4(\mathrm{~d}, J=238.3 \mathrm{~Hz}), 138.28$ 
(d, $J=2.0 \mathrm{~Hz}$ ), 133.6 (d, $J=7.6 \mathrm{~Hz}$ ), 115.4 (d, $J=23.3 \mathrm{~Hz}), 111.9$ (d, $J=24.8 \mathrm{~Hz}), 110.1$ (d, $J=8.0 \mathrm{~Hz}), 82.9,75.8,43.3,24.4,24.1$, 6.5, 5.1. ${ }^{11} \mathrm{~B}$ NMR (193 MHz, Chloroform-d) $\delta$ 37.73. ${ }^{19} \mathrm{~F}$ NMR (376 MHz, DMSO-d6) $\delta-120.86$. HRMS (ESI) m/z calcd for $\mathrm{C}_{28} \mathrm{H}_{39} \mathrm{BFNNaO}_{4} \mathrm{Si}[\mathrm{M}+\mathrm{Na}]^{+}:$534.2623, found: 534.2629. $[\alpha]_{\mathrm{D}}^{25}=-2.50(c=0.14$, DCM). HPLC (CHIRALCEL AD-H), $n$-hexane/2propanol $=95 / 5$, flow rate $0.8 \mathrm{~mL} / \mathrm{min}$, detection at $254 \mathrm{~nm}, t_{\mathrm{R}}=7.0 \mathrm{~min}$ (major), $11.6 \mathrm{~min}$ (minor).

(S)-1-benzyl-5-chloro-3-((4,4,5,5-tetramethyl-1,3,2-dioxaborolan-2-yl)methyl)-3-((triethylsilyl)oxy)indolin-2one $(\mathbf{4 g})$<smiles>CC1(C)OB(C[C@]2(OC(F)(F)F)C(=O)N(Cc3ccccc3)c3ccc(Cl)cc32)OC1(C)C</smiles>

Prepared by the general procedure $\mathrm{A}$, and $\mathbf{4 g}$ was isolated as a colorless oil, $97 \%$ ee. $(35.4 \mathrm{mg}$, $67 \%$ yield), eluent (petroleum ether/ethyl acetate $=30: 1) .{ }^{1} \mathrm{H}$ NMR $(400 \mathrm{MHz}$, DMSO-d6) $\delta 7.62$ $(\mathrm{d}, J=2.0 \mathrm{~Hz}, 1 \mathrm{H}), 7.47-7.40(\mathrm{~m}, 3 \mathrm{H}), 7.36-7.26(\mathrm{~m}, 3 \mathrm{H}), 6.93(\mathrm{~d}, J=8.4 \mathrm{~Hz}, 1 \mathrm{H}), 4.89-4.79$ $(\mathrm{m}, 2 \mathrm{H}), 1.66(\mathrm{~d}, J=14.6 \mathrm{~Hz}, 1 \mathrm{H}), 1.42(\mathrm{~d}, J=14.6 \mathrm{~Hz}, 1 \mathrm{H}), 1.01(\mathrm{~s}, 6 \mathrm{H}), 0.93(\mathrm{~s}, 6 \mathrm{H}), 0.77(\mathrm{t}, J$ $=7.9 \mathrm{~Hz}, 9 \mathrm{H}), 0.42-0.26(\mathrm{~m}, 6 \mathrm{H}) \cdot{ }^{13} \mathrm{C}$ NMR $(101 \mathrm{MHz}, \mathrm{DMSO}-\mathrm{d} 6) \delta 176.2,141.3,135.8,134.1$, $131.8,128.4,127.7,127.5,127.0,114.1,111.2,82.9,75.5,43.2,24.3,24.2,6.4,5.1 .{ }^{11}$ B NMR (193 MHz, DMSO-d6) $\delta 32.95$. HRMS (ESI) $\mathrm{m} / \mathrm{z}$ calcd for $\mathrm{C}_{28} \mathrm{H}_{39} \mathrm{BCINNaO}_{4} \mathrm{Si}[\mathrm{M}+\mathrm{Na}]^{+}: 550.2327$, found: 550.2332 . [ $\left.\alpha\right]_{D}{ }^{25}=-1.33$ ( $c=0.30$, DCM). HPLC (CHIRALCEL AD$\mathrm{H}$ ), $n$-hexane/2-propanol =95/5, flow rate $0.8 \mathrm{~mL} / \mathrm{min}$, detection at $254 \mathrm{~nm}, t_{\mathrm{R}}=6.9 \mathrm{~min}$ (major), $9.4 \mathrm{~min}$ (minor).

(S)-1-benzyl-5-bromo-3-((4,4,5,5-tetramethyl-1,3,2-dioxaborolan-2-yl)methyl)-3-((triethylsilyl)oxy)indolin-2one (4h)<smiles>CC1(C)OB(C[C@]2(OC(F)(F)F)C(=O)N(Cc3ccccc3)c3ccc(Br)cc32)OC1(C)C</smiles>
Prepared by the general procedure $\mathrm{A}$, and $\mathbf{4 h}$ was isolated as a colorless oil, $96 \%$ ee. $(31.5 \mathrm{mg}$, $55 \%$ yield), eluent (petroleum ether/ethyl acetate $=30: 1) .{ }^{1} \mathrm{H}$ NMR $(400 \mathrm{MHz}$, DMSO- $d 6) \delta 7.62$ $(\mathrm{d}, J=2.0 \mathrm{~Hz}, 1 \mathrm{H}), 7.46-7.40(\mathrm{~m}, 3 \mathrm{H}), 7.35-7.28(\mathrm{~m}, 3 \mathrm{H}), 6.92(\mathrm{~d}, J=8.3 \mathrm{~Hz}, 1 \mathrm{H}), 4.89-4.79$ $(\mathrm{m}, 2 \mathrm{H}), 1.66(\mathrm{~d}, J=14.6 \mathrm{~Hz}, 1 \mathrm{H}), 1.42(\mathrm{~d}, J=14.7 \mathrm{~Hz}, 1 \mathrm{H}), 1.01(\mathrm{~s}, 6 \mathrm{H}), 0.93(\mathrm{~s}, 6 \mathrm{H}), 0.76(\mathrm{t}, J$ $=7.9 \mathrm{~Hz}, 9 \mathrm{H}), 0.42-0.26(\mathrm{~m}, 6 \mathrm{H}) .{ }^{13} \mathrm{C}$ NMR $(101 \mathrm{MHz}, \mathrm{DMSO}-\mathrm{d} 6) \delta 176.7,141.8,136.3,134.6$, 132.3, 128.9, 128.2, 128.0, 127.5, 114.6, 111.7, 83.4, 76.0, 43.7, 24.8, 24.7, 6.9, 5.6. ${ }^{11}$ B NMR (193 MHz, DMSO-d6) $\delta 33.53$. HRMS

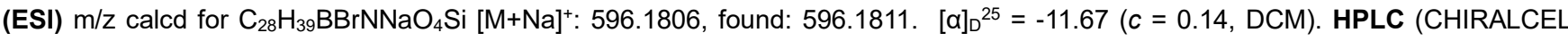
$\mathrm{AD}-\mathrm{H}$ ), $n$-hexane/2-propanol $=95 / 5$, flow rate $0.8 \mathrm{~mL} / \mathrm{min}$, detection at $254 \mathrm{~nm}, t_{\mathrm{R}}=6.9 \mathrm{~min}$ (major), $9.0 \mathrm{~min}$ (minor).

(S)-1-benzyl-6-fluoro-3-((4,4,5,5-tetramethyl-1,3,2-dioxaborolan-2-yl)methyl)-3-((triethylsilyl)oxy)indolin-2one (4i)<smiles>CC1(C)OB(C[C@]2(OC(F)(F)F)C(=O)N(Cc3ccccc3)c3cc(F)ccc32)OC1(C)C</smiles>

Prepared by the general procedure A, and $4 \mathbf{i}$ was isolated as a colorless oil, $86 \%$ ee. $(22.5 \mathrm{mg}$, $44 \%$ yield), eluent (petroleum ether/ethyl acetate $=30: 1) .{ }^{1} \mathrm{H}$ NMR $(400 \mathrm{MHz}$, DMSO-d6) $\delta 7.47$ $7.41(\mathrm{~m}, 3 \mathrm{H}), 7.37-7.27(\mathrm{~m}, 3 \mathrm{H}), 6.94-6.91(\mathrm{~m}, 1 \mathrm{H}), 6.86-6.81(\mathrm{~m}, 1 \mathrm{H}), 4.84(\mathrm{~s}, 2 \mathrm{H}), 1.63(\mathrm{~d}$ $J=14.4 \mathrm{~Hz}, 1 \mathrm{H}), 1.46(\mathrm{~d}, J=14.4 \mathrm{~Hz}, 1 \mathrm{H}), 0.97(\mathrm{~s}, 6 \mathrm{H}), 0.87(\mathrm{~s}, 6 \mathrm{H}), 0.75(\mathrm{t}, J=7.9 \mathrm{~Hz}, 9 \mathrm{H}), 0.39$

$-0.23(\mathrm{~m}, 6 \mathrm{H}) .{ }^{13} \mathrm{C}$ NMR (101 MHz, DMSO-d6) $\delta 177.3,163.4(\mathrm{~d}, J=243.1 \mathrm{~Hz}), 144.6(\mathrm{~d}, J=$ $12.2 \mathrm{~Hz}$ ), 128.1 (d, $J=2.8 \mathrm{~Hz}$ ), 125.9 (d, $J=10.2 \mathrm{~Hz}$ ), 108.6 (d, $J=22.3 \mathrm{~Hz}$ ), 98.2 (d, $J=27.9 \mathrm{~Hz}), 83.3,75.6,43.8,24.8,24.5,7.0$, 5.6. ${ }^{11}$ B NMR (193 MHz, Chloroform-d) $\delta$ 37.23. ${ }^{19} \mathrm{~F}$ NMR (376 MHz, DMSO-d6) $\delta-110.86$. HRMS (ESI) m/z calcd for $\mathrm{C}_{28} \mathrm{H}_{39} \mathrm{BFNNaO}{ }_{4} \mathrm{Si}[\mathrm{M}+\mathrm{Na}]^{+}: 534.2623$, found: 534.2613. $[\alpha]_{D}{ }^{25}=+3.86(c=0.18, \mathrm{DCM})$. HPLC (CHIRALCEL AD-H), $n$-hexane/2propanol $=95 / 5$, flow rate $0.8 \mathrm{~mL} / \mathrm{min}$, detection at $254 \mathrm{~nm}, t_{\mathrm{R}}=5.9 \mathrm{~min}$ (major), $10.9 \mathrm{~min}$ (minor).

(S)-1-benzyl-6-chloro-3-((4,4,5,5-tetramethyl-1,3,2-dioxaborolan-2-yl)methyl)-3-((triethylsilyl)oxy)indolin-2one (4j)<smiles>CC1(C)OB(C[C@]2(OC(F)(F)F)C(=O)N(Cc3ccccc3)c3cc(Cl)ccc32)OC1(C)C</smiles>

Prepared by the general procedure $\mathrm{A}$, and $\mathbf{4 j}$ was isolated as a colorless oil, $86 \%$ ee. $(24.8 \mathrm{mg}$, $47 \%$ yield), eluent (petroleum ether/ethyl acetate $=30: 1) .{ }^{1} \mathrm{H}$ NMR $(400 \mathrm{MHz}$, DMSO-d6) $\delta 7.46$ $-7.40(\mathrm{~m}, 3 \mathrm{H}), 7.37-7.27(\mathrm{~m}, 3 \mathrm{H}), 6.10-6.08(\mathrm{~m}, 2 \mathrm{H}), 4.86(\mathrm{~s}, 2 \mathrm{H}), 1.62(\mathrm{~d}, J=14.5 \mathrm{~Hz}, 1 \mathrm{H})$, $1.45(\mathrm{~d}, J=14.5 \mathrm{~Hz}, 1 \mathrm{H}), 0.97(\mathrm{~s}, 6 \mathrm{H}), 0.88(\mathrm{~s}, 6 \mathrm{H}), 0.75(\mathrm{t}, J=7.9 \mathrm{~Hz}, 9 \mathrm{H}), 0.40-0.24(\mathrm{~m}, 6 \mathrm{H})$

${ }^{13} \mathrm{C}$ NMR (101 MHz, DMSO-d6) $\delta 177.1,144.2,136.4,134.2,131.1,129.0,128.4,128.0,125.8$

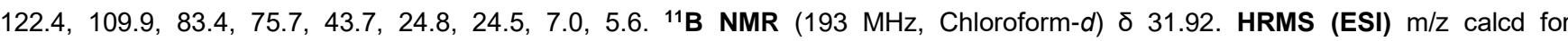




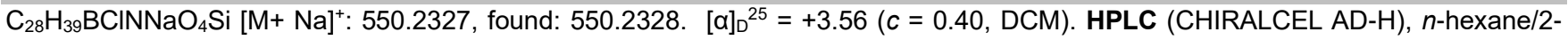
propanol $=95 / 5$, flow rate $0.8 \mathrm{~mL} / \mathrm{min}$, detection at $254 \mathrm{~nm}, t_{\mathrm{R}}=5.9 \mathrm{~min}$ (major), $10.9 \mathrm{~min}$ (minor).

(S)-1-benzyl-6-bromo-3-((4,4,5,5-tetramethyl-1,3,2-dioxaborolan-2-yl)methyl)-3-((triethylsilyl)oxy)indolin-2one (4k)<smiles>CC(F)(F)OC1(C)OB(C[C@]2(OC(F)(F)F)C(=O)N(Cc3ccccc3)c3cc(Br)ccc32)OC1(C)C</smiles>

Prepared by the general procedure A, and $\mathbf{4 k}$ was isolated as a colorless oil, $91 \%$ ee. $(23.5 \mathrm{mg}$, $41 \%$ yield), eluent (petroleum ether/ethyl acetate $=30: 1) .{ }^{1} \mathrm{H}$ NMR $(400 \mathrm{MHz}$, DMSO-d6) $\delta 7.44-$ $7.42(\mathrm{~m}, 2 \mathrm{H}), 7.37-7.19(\mathrm{~m}, 6 \mathrm{H}), 4.85(\mathrm{~s}, 2 \mathrm{H}), 1.61(\mathrm{~d}, J=14.5 \mathrm{~Hz}, 1 \mathrm{H}), 1.45(\mathrm{~d}, J=14.5 \mathrm{~Hz}$, $1 \mathrm{H}), 0.98(\mathrm{~s}, 6 \mathrm{H}), 0.88(\mathrm{~s}, 6 \mathrm{H}), 0.75(\mathrm{t}, J=7.9 \mathrm{~Hz}, 9 \mathrm{H}), 0.38-0.27(\mathrm{~m}, 6 \mathrm{H}) .{ }^{13} \mathrm{C}$ NMR $(101 \mathrm{MHz}$, DMSO-d6) $\delta 177.0,144.3,136.4,131.5,129.0,128.3,128.0,126.1,125.4,122.5,112.6,83.4$, 75.7, 43.6, 24.8, 24.5, 7.0, 5.6. ${ }^{11} \mathrm{~B}$ NMR (193 MHz, DMSO-d6) $\delta$ 32.57. HRMS (ESI) m/z calcd for $\mathrm{C}_{28} \mathrm{H}_{39} \mathrm{BBrNNaO}_{4} \mathrm{Si}[\mathrm{M}+\mathrm{Na}]^{+}$: 596.1806, found: 596.1809. [a $]_{D}^{25}=-14.17(c=0.14$, DCM). HPLC $(C H I R A L C E L ~ A D-H), n$-hexane/2-propanol = 95/5, flow rate 0.8 $\mathrm{mL} / \mathrm{min}$, detection at $254 \mathrm{~nm}, t_{\mathrm{R}}=5.8 \mathrm{~min}$ (major), $12.7 \mathrm{~min}$ (minor).

(S)-1-benzyl-7-methyl-3-((4,4,5,5-tetramethyl-1,3,2-dioxaborolan-2-yl)methyl)-3-((triethylsilyl)oxy)indolin-2one (4I)<smiles>CCO[C@]1(CB2OC(C)(C)C(C)(C)O2)C(=O)N(Cc2ccccc2)c2c(C)cccc21</smiles>

Prepared by the general procedure $\mathrm{A}$, and $\mathbf{4 I}$ was isolated as a colorless oil, $80 \%$ ee. $(29.9 \mathrm{mg}, 59 \%$ yield), eluent (petroleum ether/ethyl acetate $=30: 1) .{ }^{1} \mathrm{H}$ NMR $(400 \mathrm{MHz}$, DMSO- $d 6) \delta 7.35-7.23(\mathrm{~m}$, $6 \mathrm{H}), 7.01-6.92(\mathrm{~m}, 2 \mathrm{H}), 5.12-5.00(\mathrm{~m}, 2 \mathrm{H}), 2.17(\mathrm{~s}, 3 \mathrm{H}), 1.66-1.54(\mathrm{~m}, 2 \mathrm{H}), 0.99(\mathrm{~s}, 6 \mathrm{H}), 0.86(\mathrm{~s}$, $6 \mathrm{H}), 0.80(\mathrm{t}, J=7.9 \mathrm{~Hz}, 9 \mathrm{H}), 0.47-0.33(\mathrm{~m}, 6 \mathrm{H}) .{ }^{13} \mathrm{C}$ NMR $(101 \mathrm{MHz}$, DMSO-d6) $\delta 178.5,140.8$, $138.8,133.4,133.2,128.9,127.5,126.4,122.9,122.1,120.0,83.3,75.4,45.5,24.9,24.5,18.6,7.1$, 5.8. ${ }^{11} \mathrm{~B}$ NMR (193 MHz, DMSO-d6) $\delta$ 32.57. HRMS (ESI) m/z calcd for $\mathrm{C}_{29} \mathrm{H}_{42} \mathrm{BNNaO}_{4} \mathrm{Si}[\mathrm{M}+\mathrm{Na}]^{+}: 530.2874$, found: 530.2865 . [a] ${ }_{\mathrm{D}}{ }^{25}$ $=-11.67\left(c=0.12\right.$, DCM). HPLC $\left(\right.$ CHIRALCEL AD-H), $n$-hexane $/ 2$-propanol $=95 / 5$, flow rate $0.8 \mathrm{~mL} / \mathrm{min}$, detection at $254 \mathrm{~nm}, t_{\mathrm{R}}=$ $9.2 \mathrm{~min}$ (major), $16.5 \mathrm{~min}$ (minor).

(S)-1-benzyl-7-fluoro-3-((4,4,5,5-tetramethyl-1,3,2-dioxaborolan-2-yl)methyl)-3-((triethylsilyl)oxy)indolin-2one $(4 \mathrm{~m})$<smiles>CC1(C)OB(C[C@]2(OC(F)(F)F)C(=O)N(Cc3ccccc3)c3c(F)cccc32)OC1(C)C</smiles>

Prepared by the general procedure A, and $\mathbf{4 m}$ was isolated as a colorless oil, $90 \%$ ee. $(25.6 \mathrm{mg}, 50 \%$ yield), eluent (petroleum ether/ethyl acetate $=30: 1) .{ }^{1} \mathrm{H}$ NMR $(400 \mathrm{MHz}$, DMSO-d6) $\delta 7.38-7.25(\mathrm{~m}$, $6 \mathrm{H}), 7.19-7.14(\mathrm{~m}, 1 \mathrm{H}), 7.09-7.04(\mathrm{~m}, 1 \mathrm{H}), 4.91(\mathrm{~s}, 2 \mathrm{H}), 1.63(\mathrm{~d}, J=14.5 \mathrm{~Hz}, 1 \mathrm{H}), 1.52(\mathrm{~d}, J=14.5$ $\mathrm{Hz}, 1 \mathrm{H}), 0.97(\mathrm{~s}, 6 \mathrm{H}), 0.87(\mathrm{~s}, 6 \mathrm{H}), 0.76(\mathrm{t}, J=7.9 \mathrm{~Hz}, 9 \mathrm{H}), 0.29-0.40(\mathrm{~m}, 6 \mathrm{H}) .{ }^{13} \mathrm{C}$ NMR $(101 \mathrm{MHz}$, DMSO-d6) $\delta 177.0,147.2$ (d, $J=242.9 \mathrm{~Hz}), 135.4(\mathrm{~d}, J=2.5 \mathrm{~Hz}), 129.0$ (d, $J=8.6 \mathrm{~Hz}), 124.1$ (d, $J=$ $6.3 \mathrm{~Hz}), 120.6$ (d, $J=3.0 \mathrm{~Hz}), 117.8$ (d, $J=19.4 \mathrm{~Hz}), 83.4,76.1$ (d, $J=2.4 \mathrm{~Hz}), 45.5$ (d, $J=4.5 \mathrm{~Hz}$ ),

24.9, 24.5, 7.0, 5.6. ${ }^{11} \mathrm{~B}$ NMR (193 MHz, Chloroform- $d$ ) $\delta$ 32.00. ${ }^{19} \mathrm{~F}$ NMR (376 MHz, Chloroform- $d$ ) $\delta-134.18$. HRMS (ESI) m/z calcd for $\mathrm{C}_{28} \mathrm{H}_{39} \mathrm{BFNNaO}{ }_{4} \mathrm{Si}[\mathrm{M}+\mathrm{Na}]^{+}: 534.2623$, found: 534.2624. [ $\left.\alpha\right]_{\mathrm{D}}{ }^{25}=-4.17$ ( $c=0.14$, DCM). HPLC (CHIRALCEL AD-H), $n$-hexane/2propanol $=95 / 5$, flow rate $0.8 \mathrm{~mL} / \mathrm{min}$, detection at $254 \mathrm{~nm}, t_{\mathrm{R}}=6.3 \mathrm{~min}$ (major), $12.2 \mathrm{~min}$ (minor).

\section{(S)-1-benzyl-7-chloro-3-((4,4,5,5-tetramethyl-1,3,2-dioxaborolan-2-yl)methyl)-3-((triethylsilyl)oxy)indolin-2-} one $(4 n)$<smiles>CC1(C)OB(C[C@]2(OC(F)(F)F)C(=O)N(Cc3ccccc3)c3c(Cl)cccc32)OC1(C)C</smiles>

Prepared by the general procedure $\mathrm{A}$, and $\mathbf{4 n}$ was isolated as a colorless oil, $82 \%$ ee. $(26.9 \mathrm{mg}, 51 \%$ yield), eluent (petroleum ether/ethyl acetate $=30: 1) .{ }^{1} \mathrm{H}$ NMR $(400 \mathrm{MHz}$, DMSO-d6) $\delta 7.41-7.39(\mathrm{~m}$, $1 \mathrm{H}), 7.36-7.24(\mathrm{~m}, 6 \mathrm{H}), 6.08(\mathrm{t}, J=7.8 \mathrm{~Hz}, 1 \mathrm{H}), 5.23-5.11(\mathrm{~m}, 2 \mathrm{H}), 1.67-1.66(\mathrm{~m}, 2 \mathrm{H}), 0.99(\mathrm{~s}$, $6 \mathrm{H}), 0.88(\mathrm{~s}, 6 \mathrm{H}), 0.78(\mathrm{t}, J=7.9 \mathrm{~Hz}, 9 \mathrm{H}), 0.46-0.33(\mathrm{~m}, 6 \mathrm{H}) .{ }^{13} \mathrm{C}$ NMR $(101 \mathrm{MHz}$, DMSO-d6) $\delta$ $177.9,138.6,138.2,135.6,131.9,128.7,127.5,126.9,124.5,123.3,114.7,83.4,75.6,45.3,24.9$, 24.5, 7.0, 5.7. ${ }^{11}$ B NMR (193 MHz, DMSO-d6) $\delta$ 32.08. HRMS (ESI) m/z calcd for $\mathrm{C}_{28} \mathrm{H}_{39} \mathrm{BCINNaO}{ }_{4} \mathrm{Si}[\mathrm{M}+\mathrm{Na}]^{+}: 550.2327$, found: 550.2331. $[\alpha]_{D}^{25}=+3.75(c=0.15$, DCM). HPLC (CHIRALCEL AD-H), $n$-hexane $/ 2$-propanol $=95 / 5$, flow rate $0.8 \mathrm{~mL} / \mathrm{min}$, detection at $254 \mathrm{~nm}, t_{\mathrm{R}}=6.3 \mathrm{~min}$ (major), $10.7 \mathrm{~min}$ (minor). 
(S)-1-benzyl-7-bromo-3-((4,4,5,5-tetramethyl-1,3,2-dioxaborolan-2-yl)methyl)-3-((triethylsilyl)oxy)indolin-2one (40)<smiles>CC1(C)OB(C[C@]2(OC(F)(F)F)C(=O)N(Cc3ccccc3)c3c(Br)cccc32)OC1(C)C</smiles>

Prepared by the general procedure A, and 40 was isolated as a colorless oil, $80 \%$ ee. $(28.1 \mathrm{mg}, 49 \%$ yield), eluent (petroleum ether/ethyl acetate $=30: 1) .{ }^{1} \mathrm{H}$ NMR $(400 \mathrm{MHz}$, DMSO-d6) $\delta 7.46-7.42(\mathrm{~m}$, $2 \mathrm{H}), 7.34-7.30(\mathrm{~m}, 4 \mathrm{H}), 7.27-7.24(\mathrm{~m}, 1 \mathrm{H}), 7.01(\mathrm{t}, J=7.7 \mathrm{~Hz}, 1 \mathrm{H}), 5.28-5.15(\mathrm{~m}, 2 \mathrm{H}), 1.67-1.56$ (m, 2H), $0.99(\mathrm{~s}, 6 \mathrm{H}), 0.89(\mathrm{~s}, 6 \mathrm{H}), 0.79(\mathrm{t}, \mathrm{J}=7.9 \mathrm{~Hz}, 9 \mathrm{H}), 0.46-0.32(\mathrm{~m}, 6 \mathrm{H}) .{ }^{13} \mathrm{C}$ NMR $(101 \mathrm{MHz}$, DMSO-d6) $\delta 178.1,140.0,138.1,136.0,135.3,128.7,127.4,126.9,124.8,123.7,102.2,83.4,75.5$,

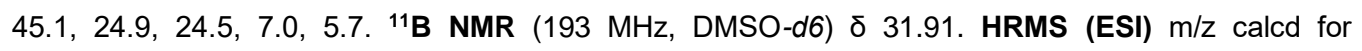
$\mathrm{C}_{28} \mathrm{H}_{39} \mathrm{BBrNNaO}_{4} \mathrm{Si}[\mathrm{M}+\mathrm{Na}]^{+}: 596.1806$, found: 596.1805. $[\alpha]_{0}{ }^{25}=-10.23(c=0.14$, DCM). HPLC (CHIRALCEL AD-H), $n$-hexane/2propanol $=95 / 5$, flow rate $0.8 \mathrm{~mL} / \mathrm{min}$, detection at $254 \mathrm{~nm}, t_{\mathrm{R}}=8.7 \mathrm{~min}$ (major), $19.3 \mathrm{~min}$ (minor).

(S)-1-benzyl-4-fluoro-3-((4,4,5,5-tetramethyl-1,3,2-dioxaborolan-2-yl)methyl)-3-((triethylsilyl)oxy)indolin-2one (4q)<smiles>CC1(C)OB(C2([O-])C(=O)N(Cc3ccccc3)c3cccc(F)c32)OC1(C)C</smiles>

Prepared by the general procedure $\mathrm{A}$, and $\mathbf{4 q}$ was isolated as a colorless oil, $80 \%$ ee. $(21.5 \mathrm{mg}, 42 \%$ yield), eluent (petroleum ether/ethyl acetate $=30: 1) .{ }^{1} \mathrm{H}$ NMR $(400 \mathrm{MHz}$, Chloroform- $d$ ) $\delta 7.43-7.41$ (m, 2H), $7.35-7.26(\mathrm{~m}, 3 \mathrm{H}), 7.14-7.09(\mathrm{~m}, 1 \mathrm{H}), 6.65(\mathrm{t}, J=8.7 \mathrm{~Hz}, 1 \mathrm{H}), 6.48(\mathrm{~d}, J=7.8 \mathrm{~Hz}, 1 \mathrm{H})$, $4.83(\mathrm{~s}, 2 \mathrm{H}), 1.03-1.89(\mathrm{~m}, 2 \mathrm{H}), 0.97(\mathrm{~s}, 6 \mathrm{H}), 0.87-0.83(\mathrm{~m}, 15 \mathrm{H}), 0.62-0.46(\mathrm{~m}, 6 \mathrm{H}) .{ }^{13} \mathrm{C} \mathrm{NMR}$ (151 MHz, Chloroform-d) $\delta 176.7,159.3(\mathrm{~d}, J=250.5 \mathrm{~Hz}$ ), 145.0 (d, $J=9.1 \mathrm{~Hz}), 130.7$ (d, $J=8.8 \mathrm{~Hz}$ ), $118.3(\mathrm{~d}, J=19.9 \mathrm{~Hz}), 110.2(\mathrm{~d}, J=21.0 \mathrm{~Hz}), 105.0$ (d, $J=2.6 \mathrm{~Hz}), 83.1,75.6,44.7,24.5,24.1,6.6,5.6 .{ }^{11} \mathrm{~B}$ NMR (193 MHz, Chloroform- $d$ ) $\delta 31.00 .{ }^{19} \mathrm{~F}$ NMR $\left(376 \mathrm{MHz}\right.$, Chloroform- $d$ ) $\delta$-118.24. HRMS (ESI) $\mathrm{m} / \mathrm{z}$ calcd for $\mathrm{C}_{28} \mathrm{H}_{39} \mathrm{BFNNaO}{ }_{4} \mathrm{Si}[\mathrm{M}+\mathrm{Na}]^{+}$: 534.2623, found: 534.2630. [a $]_{D}{ }^{25}=-5.34(c=0.13$, DCM). HPLC (CHIRALCEL AD-H), $n$-hexane/2-propanol $=95 / 5$, flow rate 0.8 $\mathrm{mL} / \mathrm{min}$, detection at $254 \mathrm{~nm}, t_{\mathrm{R}}=12.2 \mathrm{~min}$ (major), $27.9 \mathrm{~min}$ (minor).

(S)-1-benzyl-4-chloro-3-((4,4,5,5-tetramethyl-1,3,2-dioxaborolan-2-yl)methyl)-3-((triethylsilyl)oxy)indolin-2one (4r)<smiles>CC1(C)OB(C2([O-])C(=O)Nc3cccc(Cl)c32)OC1(C)C</smiles>

$\mathrm{Bn}$

Prepared by the general procedure $\mathrm{A}$, and $\mathbf{4 r}$ was isolated as a colorless oil, $97 \%$ ee. $(20.6 \mathrm{mg}, 39 \%$ yield), eluent (petroleum ether/ethyl acetate $=30: 1) .{ }^{1} \mathrm{H}$ NMR $(400 \mathrm{MHz}$, Chloroform-d) $\delta 7.34-7.33$ $(\mathrm{m}, 2 \mathrm{H}), 7.27-7.18(\mathrm{~m}, 3 \mathrm{H}), 6.98(\mathrm{t}, J=8.0 \mathrm{~Hz}, 1 \mathrm{H}), 6.82(\mathrm{~d}, J=8.2 \mathrm{~Hz}, 1 \mathrm{H}), 6.55(\mathrm{~d}, J=7.7 \mathrm{~Hz}, 1 \mathrm{H})$, $4.81-4.75(\mathrm{~m}, 2 \mathrm{H}), 2.18(\mathrm{~d}, J=15.0 \mathrm{~Hz}, 1 \mathrm{H}), 1.84(\mathrm{~d}, J=15.0 \mathrm{~Hz}, 1 \mathrm{H}), 0.89(\mathrm{~s}, 6 \mathrm{H}), 0.79(\mathrm{t}, J=7.9$ $\mathrm{Hz}, 9 \mathrm{H}), 0.74(\mathrm{~s}, 6 \mathrm{H}), 0.48-0.42(\mathrm{~m}, 6 \mathrm{H}) .{ }^{13} \mathrm{C}$ NMR $(151 \mathrm{MHz}$, Chloroform-d) $\delta$ 176.6, 144.7, 135.6, 131.4, 129.8, 129.3, 128.6, 127.7, 127.6, 123.7, 107.3, 83.0, 44.6, 24.5, 24.1, 6.8, 5.7. ${ }^{11}$ B NMR (193 MHz, Chloroform-d) $\delta 33.70$. HRMS (ESI) $\mathrm{m} / \mathrm{z}$ calcd for $\mathrm{C}_{28} \mathrm{H}_{39} \mathrm{BCINNaO}_{4} \mathrm{Si}\left[\mathrm{M}+\mathrm{Na}^{+} \text {: 550.2327, found: 550.2321. [a }\right]_{0}{ }_{2}^{25}=-4.44(c=0.16$, DCM). HPLC (CHIRALCEL AD-H), $n$-hexane/2-propanol $=95 / 5$, flow rate $0.8 \mathrm{~mL} / \mathrm{min}$, detection at $254 \mathrm{~nm}, t_{R}=5.9 \mathrm{~min}$ (major), $7.6 \mathrm{~min}$ (minor).

(S)-1-benzyl-4-bromo-3-((4,4,5,5-tetramethyl-1,3,2-dioxaborolan-2-yl)methyl)-3-((triethylsilyl)oxy)indolin-2one (4s)<smiles>CC1(C)OB(C2([O-])C(=O)Nc3cccc(Br)c32)OC1(C)C</smiles>

$\mathrm{Bn}$

Prepared by the general procedure A, and $\mathbf{4 s}$ was isolated as a colorless oil, $99 \%$ ee. $(30.3 \mathrm{mg}, 53 \%$ yield), eluent (petroleum ether/ethyl acetate $=30: 1) .{ }^{1} \mathrm{H}$ NMR $(400 \mathrm{MHz}$, Chloroform- $d$ ) $\delta 7.34-7.32$ $(\mathrm{m}, 2 \mathrm{H}), 7.26-7.19(\mathrm{~m}, 3 \mathrm{H}), 7.01(\mathrm{~d}, J=8.1 \mathrm{~Hz}, 1 \mathrm{H}), 6.90(\mathrm{t}, J=7.9 \mathrm{~Hz}, 1 \mathrm{H}), 6.52(\mathrm{~d}, J=7.7 \mathrm{~Hz}, 1 \mathrm{H})$, $4.81-4.71(\mathrm{~m}, 2 \mathrm{H}), 2.27(\mathrm{~d}, J=15.0 \mathrm{~Hz}, 1 \mathrm{H}), 1.80(\mathrm{~d}, J=15.0 \mathrm{~Hz}, 1 \mathrm{H}), 0.89(\mathrm{~s}, 6 \mathrm{H}), 0.79(\mathrm{t}, J=7.9$ $\mathrm{Hz}, 9 \mathrm{H}), 0.74(\mathrm{~s}, 6 \mathrm{H}), 0.49-0.43(\mathrm{~m}, 6 \mathrm{H}) .{ }^{13} \mathrm{C}$ NMR $(151 \mathrm{MHz}$, Chloroform-d) $\delta$ 176.6, 144.8, 135.6, 131.0, 130.0, 128.6, 127.7, 127.6, 126.8, 119.4, 107.8, 83.0, 44.5, 24.5, 24.1, 6.8, 5.8. ${ }^{11}$ B NMR (193 MHz, Chloroform-d) $\delta 31.72$. HRMS (ESI) $\mathrm{m} / \mathrm{z}$ calcd for $\mathrm{C}_{28} \mathrm{H}_{39} \mathrm{BBrNNaO}_{4} \mathrm{Si}\left[\mathrm{M}+\mathrm{Na}^{+} \text {: 596.1806, found: 596.1812. [a }\right]_{\mathrm{D}}{ }^{25}=-9.86(c=0.21$, DCM). HPLC (CHIRALCEL AD-H), $n$-hexane/2-propanol $=95 / 5$, flow rate $0.8 \mathrm{~mL} / \mathrm{min}$, detection at $254 \mathrm{~nm}, t_{\mathrm{R}}=10.4 \mathrm{~min}$ (major), $19.8 \mathrm{~min}$ (minor). 


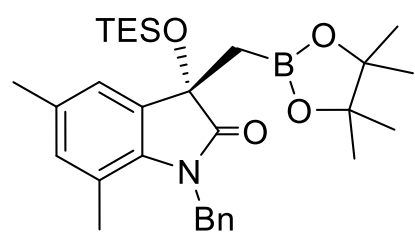

Prepared by the general procedure $\mathrm{A}$, and $\mathbf{4 t}$ was isolated as a colorless oil, $90 \%$ ee. (32.8 $\mathrm{mg}$, $63 \%$ yield), eluent (petroleum ether/ethyl acetate $=30: 1) .{ }^{1} \mathrm{H}$ NMR $(400 \mathrm{MHz}$, DMSO-d6) $\delta 7.37-$ $7.27(\mathrm{~m}, 5 \mathrm{H}), 7.07(\mathrm{~s}, 1 \mathrm{H}), 6.81(\mathrm{~s}, 1 \mathrm{H}), 5.10-4.97(\mathrm{~m}, 2 \mathrm{H}), 2.21(\mathrm{~s}, 3 \mathrm{H}), 2.13(\mathrm{~s}, 3 \mathrm{H}), 1.63-1.57$ $(\mathrm{m}, 2 \mathrm{H}), 1.00(\mathrm{~s}, 6 \mathrm{H}), 0.89(\mathrm{~s}, 6 \mathrm{H}), 0.82(\mathrm{t}, J=7.9 \mathrm{~Hz}, 9 \mathrm{H}), 0.47-0.36(\mathrm{~m}, 6 \mathrm{H}) .{ }^{13} \mathrm{C}$ NMR (151 $\mathrm{MHz}$, DMSO-d6) $\delta 178.3,138.8,138.3,133.5,133.2,131.7,128.9,127.4,126.4,122.8,119.6$,

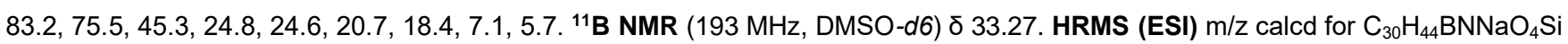
$[\mathrm{M}+\mathrm{Na}]^{+}:$: 544.3030, found: 544.3037. $[\alpha]_{D}^{25}=+3.86(c=0.16$, DCM). HPLC (CHIRALCEL AD-H), $n$-hexane/2-propanol = 95/5, flow rate $0.8 \mathrm{~mL} / \mathrm{min}$, detection at $254 \mathrm{~nm}, t_{\mathrm{R}}=7.0 \mathrm{~min}$ (major), $11.6 \mathrm{~min}$ (minor).

(S)-1-phenyl-3-((4,4,5,5-tetramethyl-1,3,2-dioxaborolan-2-yl)methyl)-3-((triethylsilyl)oxy)indolin-2-one (4u)<smiles>CC1(C)OB(C[C@]2(OC(F)(F)F)C(=O)N(c3ccccc3)c3ccccc32)OC1(C)C</smiles>
Prepared by the general procedure $\mathrm{A}$, and $\mathbf{4 u}$ was isolated as a colorless oil, $80 \%$ ee. $(35.9 \mathrm{mg}, 75 \%$ yield), eluent (petroleum ether/ethyl acetate $=30: 1) .{ }^{1} \mathrm{H}$ NMR $(400 \mathrm{MHz}$, Chloroform-d) $\delta 7.54-7.37$ $(\mathrm{m}, 6 \mathrm{H}), 7.18(\mathrm{t}, J=7.6 \mathrm{~Hz}, 1 \mathrm{H}), 7.05(\mathrm{t}, J=7.4 \mathrm{~Hz}, 1 \mathrm{H}), 6.76(\mathrm{~d}, J=7.8 \mathrm{~Hz}, 1 \mathrm{H}), 1.88-1.79(\mathrm{~m}, 2 \mathrm{H})$, $1.00(\mathrm{~s}, 6 \mathrm{H}), 0.88-0.84(\mathrm{~m}, 15 \mathrm{H}), 0.60-0.42(\mathrm{~m}, 6 \mathrm{H}) .{ }^{13} \mathrm{C}$ NMR $(101 \mathrm{MHz}$, Chloroform-d) $\delta 175.7$, 142.2, 133.8, 131.4, 128.4, 127.9, 126.8, 125.4, 123.1, 121.9, 108.1, 82.2, 75.1, 23.7, 23.2, 5.8, 4.7. ${ }^{11} \mathrm{~B}$ NMR (193 MHz, Chloroform- $d$ ) $\delta$ 31.94. HRMS (ESI) m/z calcd for $\mathrm{C}_{27} \mathrm{H}_{38} \mathrm{BNNaO}_{4} \mathrm{Si}[\mathrm{M}+\mathrm{Na}]^{+}: 502.2560$, found: 502.2558 . [a] ${ }_{\mathrm{D}}^{25}$ $=+6.57(c=0.28, \mathrm{DCM})$. HPLC $\left(\right.$ CHIRALCEL AD-H), $n$-hexane/2-propanol $=95 / 5$, flow rate $0.8 \mathrm{~mL} / \mathrm{min}$, detection at $254 \mathrm{~nm}, t_{\mathrm{R}}=5.6$ $\min$ (major), $11.6 \mathrm{~min}$ (minor).

(S)-1-(4-methoxybenzyl)-3-((4,4,5,5-tetramethyl-1,3,2-dioxaborolan-2-yl)methyl)-3-((triethylsilyl)oxy)indolin -2-one (4v)<smiles>[R15]N1C(=O)[C@](CB2OC(C)(C)C(C)(C)O2)(OS(F)(F)F)c2ccccc21</smiles>

Prepared by the general procedure $\mathrm{A}$, and $\mathbf{4 v}$ was isolated as a colorless oil, $83 \%$ ee. $(31.4 \mathrm{mg}, 60 \%$ yield), eluent (petroleum ether/ethyl acetate $=30: 1) .{ }^{1} \mathbf{H}$ NMR $(400 \mathrm{MHz}$, Chloroform- $d) \delta 7.41(\mathrm{dd}, J=$ 7.3, $1.3 \mathrm{~Hz}, 1 \mathrm{H}), 7.34(\mathrm{~d}, J=8.6 \mathrm{~Hz}, 2 \mathrm{H}), 7.14(\mathrm{td}, J=7.7,1.3 \mathrm{~Hz}, 1 \mathrm{H}), 6.97(\mathrm{t}, J=7.3 \mathrm{~Hz}, 1 \mathrm{H}), 6.84$ $(\mathrm{d}, J=8.7 \mathrm{~Hz}, 2 \mathrm{H}), 6.69(\mathrm{~d}, J=7.8 \mathrm{~Hz}, 1 \mathrm{H}), 4.83-4.73(\mathrm{~m}, 2 \mathrm{H}), 3.16(\mathrm{~s}, 3 \mathrm{H}), 1.79-1.66(\mathrm{~m}, 2 \mathrm{H})$, $1.00(\mathrm{~s}, 6 \mathrm{H}), 0.89(\mathrm{~s}, 6 \mathrm{H}), 0.82(\mathrm{t}, J=7.9 \mathrm{~Hz}, 9 \mathrm{H}), 0.50-0.34(\mathrm{~m}, 6 \mathrm{H}) .{ }^{13} \mathrm{C}$ NMR $(151 \mathrm{MHz}$, Chloroform-d) $\delta 177.6,159.0,142.6,132.8,129.2,129.2,128.9,128.1,124.0,122.3,113.9,113.9,108.8,83.0,76.0,55.2,43.7$, 24.6, 24.3, 6.7, 5.7. ${ }^{11} \mathrm{~B}$ NMR (193 MHz, Chloroform-d) $\delta$ 32.16. HRMS (ESI) m/z calcd for $\mathrm{C}_{29} \mathrm{H}_{42} \mathrm{BNNaO}_{5} \mathrm{Si}[\mathrm{M}+\mathrm{Na}]^{+}: 546.2823$, found: 546.2820. $[\alpha]_{D}^{25}=+5.27(c=0.13$, DCM). HPLC (CHIRALCEL AD-H), $n$-hexane $/ 2$-propanol $=95 / 5$, flow rate $0.8 \mathrm{~mL} / \mathrm{min}$, detection at $254 \mathrm{~nm}, t_{\mathrm{R}}=7.8 \mathrm{~min}$ (major), $13.2 \mathrm{~min}$ (minor).

(S)-1-methyl-3-((4,4,5,5-tetramethyl-1,3,2-dioxaborolan-2-yl)methyl)-3-((triethylsilyl)oxy)indolin-2-one (4w)<smiles>CN1C(=O)[C@](CB2OC(C)(C)C(C)(C)O2)(OS(F)(F)F)c2ccccc21</smiles>
Prepared by the general procedure A, and $\mathbf{4 w}$ was isolated as a colorless oil, $75 \%$ ee. $(27.9 \mathrm{mg}, 67 \%$ yield), eluent (petroleum ether/ethyl acetate $=30: 1) .{ }^{1} \mathbf{H}$ NMR $(400 \mathrm{MHz}$, Chloroform- $d) \delta 7.42(\mathrm{dd}, \mathrm{J}=$ 7.4, $1.3 \mathrm{~Hz}, 1 \mathrm{H}$ ), 7.26 (td, J = 7.7, $1.3 \mathrm{~Hz}, 1 \mathrm{H}), 7.02$ (td, J = 7.6, $1.0 \mathrm{~Hz}, 1 \mathrm{H}), 6.75$ (d, J = 7.7 Hz, 1H), $3.16(\mathrm{~s}, 3 \mathrm{H}), 1.73-1.61(\mathrm{~m}, 2 \mathrm{H}), 1.00(\mathrm{~s}, 6 \mathrm{H}), 0.94(\mathrm{~s}, 6 \mathrm{H}), 0.83(\mathrm{t}, J=7.9 \mathrm{~Hz}, 9 \mathrm{H}), 0.49-0.33(\mathrm{~m}$, $6 \mathrm{H}) .{ }^{13} \mathrm{C}$ NMR $(151 \mathrm{MHz}$, Chloroform-d) $\delta 177.5,143.2,132.6,129.1,124.0,122.4,107.7,83.0,76.1$, 26.0, 24.6, 24.4, 6.7, 5.6. ${ }^{11} \mathrm{~B}$ NMR (193 MHz, Chloroform-d) $\delta 31.90$. HRMS (ESI) $\mathrm{m} / \mathrm{z}$ calcd for $\mathrm{C}_{22} \mathrm{H}_{36} \mathrm{BNNaO}{ }_{4} \mathrm{Si}[\mathrm{M}+\mathrm{Na}]^{+}$: 440.2403, found:440.2408. [ $\alpha]_{D}{ }^{25}=-6.67(c=0.13$, DCM). HPLC (Chiralpak IC), $n$-hexane $/ 2$-propanol $=99 / 1$, flow rate $0.8 \mathrm{~mL} / \mathrm{min}$, detection at $254 \mathrm{~nm}, t_{\mathrm{R}}=11.5 \mathrm{~min}$ (minor), $12.6 \mathrm{~min}$ (major).

\section{(S)-1-benzyl-3-((R)-1-(4,4,5,5-tetramethyl-1,3,2-dioxaborolan-2-yl)ethyl)-3-((triethylsilyl)oxy)indolin-2-one} (6a)<smiles>CC(B1OC(C)(C)C(C)(C)O1)[C@]1(O)C(=O)Nc2ccccc21</smiles>

$\mathrm{Bn}$
Prepared by the general procedure $B$, and 6 a was isolated as a colorless oil, $>20: 1$ d.r., $86 \%$ ee. $(33.0$ $\mathrm{mg}, 65 \%$ yield), eluent (petroleum ether/ethyl acetate $=30: 1) .{ }^{1} \mathrm{H}$ NMR $(400 \mathrm{MHz}$, Chloroform- $d$ ) $\delta 7.44$ 
- $7.26(\mathrm{~m}, 6 \mathrm{H}), 7.15(\mathrm{t}, J=7.7 \mathrm{~Hz}, 1 \mathrm{H}), 6.96(\mathrm{t}, J=7.5 \mathrm{~Hz}, 1 \mathrm{H}), 6.70(\mathrm{~d}, J=7.8 \mathrm{~Hz}, 1 \mathrm{H}), 4.92(\mathrm{~d}, J=15.5 \mathrm{~Hz}, 1 \mathrm{H}), 4.72(\mathrm{~d}, J=15.5$ $\mathrm{Hz}, 1 \mathrm{H}), 1.96-1.91(\mathrm{~m}, 1 \mathrm{H}), 1.22(\mathrm{~d}, J=7.5 \mathrm{~Hz}, 2 \mathrm{H}), 0.94(\mathrm{~d}, J=18.7 \mathrm{~Hz}, 12 \mathrm{H}), 0.81(\mathrm{t}, J=8.0 \mathrm{~Hz}, 9 \mathrm{H}), 0.47-0.31(\mathrm{~m}, 6 \mathrm{H}) .{ }^{13} \mathrm{C}$ NMR $(151 \mathrm{MHz}$, Chloroform-d) $\delta 177.6,143.1,136.1,131.8,128.9,128.5,128.1,127.5,124.5,122.1,108.8,82.9,78.3,44.6,24.4$, 9.2, 6.8, 5.7. ${ }^{11}$ B NMR (193 MHz, Chloroform-d) $\delta$ 32.54. HRMS (ESI) m/z calcd for $\mathrm{C}_{29} \mathrm{H}_{42} \mathrm{BNNaO}_{4} \mathrm{Si}[\mathrm{M}+\mathrm{Na}]^{+}: 530.2874$, found: 530.2882. $[\alpha]_{D}{ }^{25}=+2.73(c=0.22$, DCM $)$. HPLC (CHIRALCEL AD-H), $n$-hexane/2-propanol $=95 / 5$, flow rate $0.8 \mathrm{~mL} / \mathrm{min}$, detection at $254 \mathrm{~nm}, t_{\mathrm{R}}=6.6 \mathrm{~min}$ (major), $18.1 \mathrm{~min}$ (minor).

\section{(S)-1-benzyl-3-((R)-1-(4,4,5,5-tetramethyl-1,3,2-dioxaborolan-2-yl)butyl)-3-((triethylsilyl)oxy)indolin-2-one} (6b)<smiles>CCC[C@H](B1OC(C)(C)C(C)(C)O1)[C@]1(OC(F)(F)F)C(=O)N(Cc2ccccc2)c2ccccc21</smiles>

Prepared by the general procedure $B$, and $\mathbf{6} \mathbf{b}$ was isolated as a colorless oil, $>20: 1$ d.r., $93 \%$ ee. (17.1 $\mathrm{mg}, 32 \%$ yield), eluent (petroleum ether/ethyl acetate $=30: 1) .{ }^{1} \mathrm{H}$ NMR $(400 \mathrm{MHz}$, Chloroform- $d) \delta 7.43$ $-7.40(\mathrm{~m}, 2 \mathrm{H}), 7.36(\mathrm{~d}, J=7.2 \mathrm{~Hz}, 1 \mathrm{H}), 7.31(\mathrm{t}, J=7.1 \mathrm{~Hz}, 2 \mathrm{H}), 7.27-7.25(\mathrm{~m}, 1 \mathrm{H}), 7.15(\mathrm{t}, J=7.6$ $\mathrm{Hz}, 1 \mathrm{H}), 6.97(\mathrm{t}, J=7.5 \mathrm{~Hz}, 1 \mathrm{H}), 6.69(\mathrm{~d}, J=7.8 \mathrm{~Hz}, 1 \mathrm{H}), 4.90-4.73(\mathrm{~m}, 2 \mathrm{H}), 1.81-1.77(\mathrm{~m}, 1 \mathrm{H})$ $1.63-1.58(\mathrm{~m}, 2 \mathrm{H}), 1.47-1.42(\mathrm{~m}, 1 \mathrm{H}), 1.29-1.18(\mathrm{~m}, 1 \mathrm{H}), 1.00(\mathrm{~d}, J=6.0 \mathrm{~Hz}, 12 \mathrm{H}), 0.89(\mathrm{t}, J=7.2$ $\mathrm{Hz}, 3 \mathrm{H}), 0.80(\mathrm{t}, J=7.9 \mathrm{~Hz}, 9 \mathrm{H}), 0.42-0.30(\mathrm{~m}, 6 \mathrm{H}) .{ }^{13} \mathrm{C}$ NMR $(101 \mathrm{MHz}$, Chloroform- $d) \delta 177.3$, $142.8,136.1,132.0,128.9,128.6,128.2,127.5,124.7,122.3,108.7,82.9,78.5,44.4,26.8,24.6,24.5$ 23.2, 14.4, 6.8, 5.7. ${ }^{11} \mathrm{~B}$ NMR (193 MHz, Chloroform-d) $\delta 32.88$. HRMS (ESI) $\mathrm{m} / \mathrm{z}$ calcd for $\mathrm{C}_{31} \mathrm{H}_{46} \mathrm{BNNaO}_{4} \mathrm{Si}[\mathrm{M}+\mathrm{Na}]^{+}: 558.3187$ found: 558.3188. $[\alpha]_{D}{ }^{25}=+21.33(c=0.10, \mathrm{DCM})$. HPLC (CHIRALCEL AD-H), $n$-hexane/2-propanol $=95 / 5$, flow rate $0.8 \mathrm{~mL} / \mathrm{min}$ detection at $254 \mathrm{~nm}, t_{\mathrm{R}}=5.2 \mathrm{~min}$ (major), $14.9 \mathrm{~min}$ (minor).

\section{(S)-1-benzyl-3-((R)-3-phenyl-1-(4,4,5,5-tetramethyl-1,3,2-dioxaborolan-2-yl)propyl)-3-((triethylsilyl)oxy)}

\section{indolin-2-one $(6 c)$}

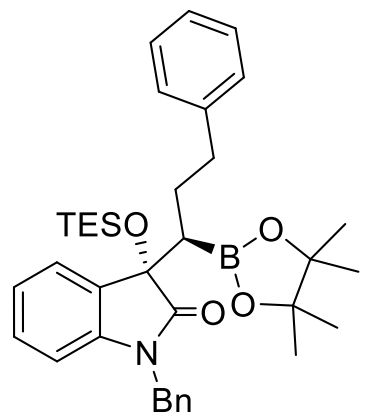

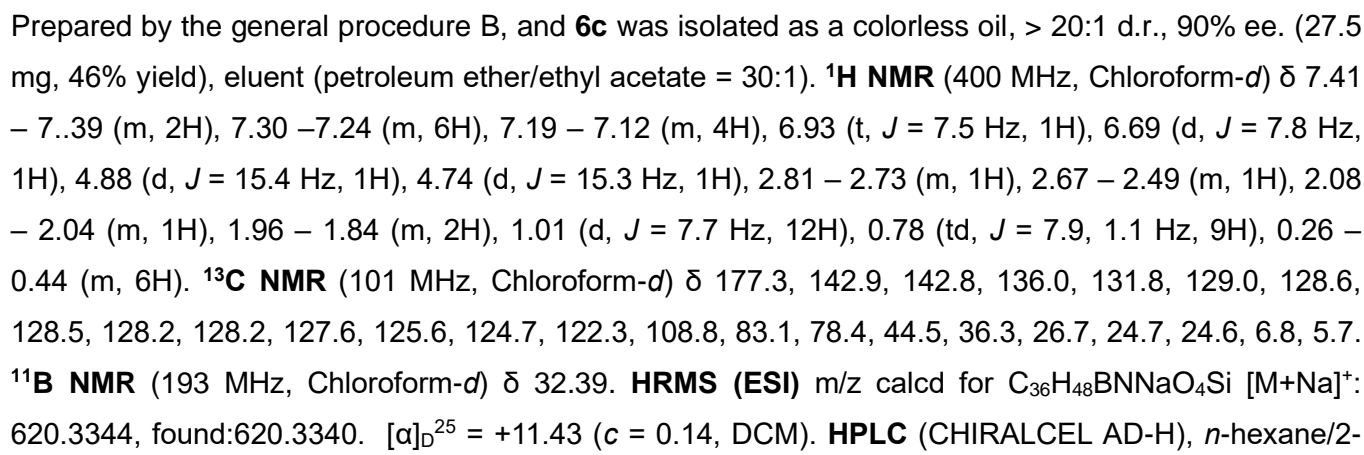
propanol $=95 / 5$, flow rate $0.8 \mathrm{~mL} / \mathrm{min}$, detection at $254 \mathrm{~nm}, t_{\mathrm{R}}=5.8 \mathrm{~min}$ (major), $12.3 \mathrm{~min}$ (minor).

(S)-1-benzyl-3-((R)-1-(4,4,5,5-tetramethyl-1,3,2-dioxaborolan-2-yl)but-3-en-1-yl)-3-((triethylsilyl)oxy)indolin2-one (6d)<smiles>C=CC[C@H](B1OC(C)(C)C(C)(C)O1)[C@H]1C(=O)N(C)c2ccccc21</smiles>

$\mathrm{Bn}$

Prepared by the general procedure B, and $6 \mathbf{d}$ was isolated as a colorless oil, $>20: 1$ d.r., $99 \%$ ee. (30.4 $\mathrm{mg}, 57 \%$ yield), eluent (petroleum ether/ethyl acetate $=30: 1) .{ }^{1} \mathrm{H}$ NMR $(400 \mathrm{MHz}$, Chloroform- $d) \delta 7.43$ - $7.41(\mathrm{~m}, 2 \mathrm{H}), 7.37-7.29(\mathrm{~m}, 4 \mathrm{H}), 7.19-7.14(\mathrm{~m}, 1 \mathrm{H}), 6.98(\mathrm{t}, J=7.5 \mathrm{~Hz}, 1 \mathrm{H}), 6.72(\mathrm{~d}, J=7.8 \mathrm{~Hz}$, $1 \mathrm{H}), 5.91-5.81(\mathrm{~m}, 1 \mathrm{H}), 5.06-5.01(\mathrm{~m}, 1 \mathrm{H}), 4.93-4.89(\mathrm{~m}, 2 \mathrm{H}), 4.72(\mathrm{~d}, J=15.4 \mathrm{~Hz}, 1 \mathrm{H}), 2.56-$ $2.51(\mathrm{~m}, 1 \mathrm{H}), 2.43-2.35(\mathrm{~m}, 1 \mathrm{H}), 1.91(\mathrm{dd}, \mathrm{J}=11.8,3.2 \mathrm{~Hz}, 1 \mathrm{H}), 0.97(\mathrm{~d}, J=2.6 \mathrm{~Hz}, 12 \mathrm{H}), 0.80(\mathrm{t}, J=$ $7.9 \mathrm{~Hz}, 9 \mathrm{H}), 0.46-0.30(\mathrm{~m}, 6 \mathrm{H}) .{ }^{13} \mathrm{C}$ NMR $(101 \mathrm{MHz}$, Chloroform-d) $\delta 177.2,142.7,138.7,136.0$, 129.0, 128.6, 128.2, 127.6, 124.8, 122.4, 114.9, 108.8, 83.1, 78.2, 44.5, 28.9, 24.6, 24.6, 6.8, 5.7. ${ }^{11} \mathrm{~B}$ NMR (193 MHz, Chloroform- $d$ ) $\delta$ 32.19. HRMS (ESI) m/z calcd for $\mathrm{C}_{31} \mathrm{H}_{44} \mathrm{BNNaO}_{4} \mathrm{Si}[\mathrm{M}+\mathrm{Na}]^{+}: 556.3030$, found:556.3029. [ $\left.\alpha\right]_{\mathrm{D}}{ }^{25}=$ $+6.00(c=0.10, \mathrm{DCM})$. HPLC (CHIRALCEL AD-H), $n$-hexane/2-propanol $=95 / 5$, flow rate $0.8 \mathrm{~mL} / \mathrm{min}$, detection at $254 \mathrm{~nm}, t_{\mathrm{R}}=5.6$ $\min$ (major), $17.9 \mathrm{~min}$ (minor). 
<smiles>CC(C)=CC[C@@H](B1OC(C)(C)C(C)(C)O1)[C@]1(OC(F)(F)F)C(=O)N(Cc2ccccc2)c2ccccc21</smiles>

Prepared by the general procedure $B$, and 6 e was isolated as a colorless oil, $>20: 1$ d.r., $99 \%$ ee. (25.3 $\mathrm{mg}, 45 \%$ yield), eluent (petroleum ether/ethyl acetate = 30:1). ${ }^{1} \mathrm{H}$ NMR $(400 \mathrm{MHz}$, Chloroform- $d$ ) $\delta 7.43$ $-7.41(\mathrm{~m}, 2 \mathrm{H}), 7.37(\mathrm{~d}, \mathrm{~J}=7.3 \mathrm{~Hz}, 1 \mathrm{H}), 7.32-7.23(\mathrm{~m}, 3 \mathrm{H}), 7.16(\mathrm{td}, J=7.7,1.3 \mathrm{~Hz}, 1 \mathrm{H}), 6.98(\mathrm{t}, J=$ $7.5 \mathrm{~Hz}, 1 \mathrm{H}), 6.71(\mathrm{~d}, J=7.8 \mathrm{~Hz}, 1 \mathrm{H}), 5.13(\mathrm{t}, J=6.9 \mathrm{~Hz}, 1 \mathrm{H}), 4.88-4.76(\mathrm{~m}, 2 \mathrm{H}), 2.38-2.20(\mathrm{~m}, 2 \mathrm{H})$, 1.80 (dd, $J=11.7,3.6 \mathrm{~Hz}, 1 \mathrm{H}), 1.63(\mathrm{~s}, 3 \mathrm{H}), 1.57(\mathrm{~s}, 3 \mathrm{H}), 1.00(\mathrm{~d}, J=3.2 \mathrm{~Hz}, 12 \mathrm{H}), 0.80$ (t, $J=7.9 \mathrm{~Hz}$, $9 \mathrm{H}), 0.44-0.27(\mathrm{~m}, 6 \mathrm{H}) .{ }^{13} \mathrm{C}$ NMR $(101 \mathrm{MHz}$, Chloroform-d) $\delta 177.2,142.7,136.1,131.8,131.7$, 128.9, 128.6, 128.2, 127.6, 124.7, 124.3, 122.3, 108.8, 83.0, 78.5, 44.4, 25.8, 24.6, 24.5, 23.5, 17.8, 6.8, 5.7. ${ }^{11} \mathrm{~B}$ NMR (193 MHz, Chloroform-d) $\delta$ 33.16. HRMS (ESI) $\mathrm{m} / \mathrm{z}$ calcd for $\mathrm{C}_{33} \mathrm{H}_{48} \mathrm{BNNaO}_{4} \mathrm{Si}$ $[\mathrm{M}+\mathrm{Na}]^{+}:$584.3344, found:584.3346. $[\alpha]_{D}{ }^{25}=+9.67(c=0.16$, DCM). HPLC (CHIRALCEL AD-H), $n$-hexane/2-propanol = 95/5, flow rate $0.8 \mathrm{~mL} / \mathrm{min}$, detection at $254 \mathrm{~nm}, t_{\mathrm{R}}=4.9 \mathrm{~min}$ (major), $17.8 \mathrm{~min}$ (minor).

\section{(S)-1-benzyl-3-((R)-3-((tert-butyldimethylsilyl)oxy)-1-(4,4,5,5-tetramethyl-1,3,2-dioxaborolan-2-yl)propyl)-3- ((triethylsilyl)oxy)indolin-2-one (6f)}<smiles>CC(C)(C)OCCC(B1OC(C)(C)C(C)(C)O1)C1(OC(F)(F)F)C(=O)N(c2ccccc2)c2ccccc21</smiles>

$\mathrm{Bn}$

Prepared by the general procedure $B$, and $6 f$ was isolated as a colorless oil, $>20: 1$ d.r., $85 \%$ ee. (34.5 $\mathrm{mg}, 53 \%$ yield), eluent (petroleum ether/ethyl acetate $=30: 1) .{ }^{1} \mathbf{H}$ NMR $(400 \mathrm{MHz}$, Chloroform- $d$ ) $\delta 7.43$ - $7.41(\mathrm{~m}, 2 \mathrm{H}), 7.36-7.25(\mathrm{~m}, 4 \mathrm{H}), 7.15(\mathrm{t}, J=7.7 \mathrm{~Hz}, 1 \mathrm{H}), 6.96(\mathrm{t}, J=7.5 \mathrm{~Hz}, 1 \mathrm{H}), 6.70(\mathrm{~d}, J=7.8$ $\mathrm{Hz}, 1 \mathrm{H}), 4.89(\mathrm{~d}, J=15.3 \mathrm{~Hz}, 1 \mathrm{H}), 4.72(\mathrm{~d}, J=15.4 \mathrm{~Hz}, 1 \mathrm{H}), 3.74-3.69(\mathrm{~m}, 1 \mathrm{H}), 3.59-3.53(\mathrm{~m}, 1 \mathrm{H})$, $2.04-2.00(\mathrm{~m}, 1 \mathrm{H}), 1.86-1.79(\mathrm{~m}, 2 \mathrm{H}), 0.96(\mathrm{~d}, J=8.3 \mathrm{~Hz}, 12 \mathrm{H}), 0.90(\mathrm{~s}, 9 \mathrm{H}), 0.79(\mathrm{t}, J=7.9 \mathrm{~Hz}$, $9 \mathrm{H}), 0.42-0.29(\mathrm{~m}, 6 \mathrm{H}), 0.04(\mathrm{~s}, 6 \mathrm{H}) .{ }^{13} \mathrm{C}$ NMR (101 MHz, Chloroform-d) $\delta 177.3,142.9,136.1,131.8$, 129.0, 128.6, 128.2, 127.5, 124.7, 122.3, 108.8, 83.0, 78.1, 63.8, 44.6, 28.0, 26.1, 24.58, 24.5, 18.4, 6.8, 5.6, -5.1, -5.2. ${ }^{11} \mathrm{~B}$ NMR (193 MHz, Chloroform-d) $\delta$ 31.91. HRMS (ESI) m/z calcd for $\mathrm{C}_{36} \mathrm{H}_{58} \mathrm{BNNaO}_{5} \mathrm{Si}_{2}[\mathrm{M}+\mathrm{Na}]^{+}: 674.3846$, found:674.3844. $[\alpha]_{D}{ }^{25}=-1.56(c=0.12$, DCM). HPLC (CHIRALCEL AD-H), $n$-hexane/2-propanol $=95 / 5$, flow rate $0.8 \mathrm{~mL} / \mathrm{min}$, detection at $254 \mathrm{~nm}, t_{\mathrm{R}}=6.7 \mathrm{~min}$ (major), $8.8 \mathrm{~min}$ (minor).

\subsection{Cu-catalyzed 1,2-addition reaction of diboryl methane at $1 \mathrm{mmol}$ scale}<smiles>O=C1C(=O)N(Cc2ccccc2)c2ccccc21</smiles>

$1 \mathrm{a}$
(1) $\mathrm{Cu}\left(\mathrm{CH}_{3} \mathrm{CN}\right)_{4} \mathrm{PF}_{6}(10 \mathrm{~mol} \%)$, L1 (20 mol\%), LiOtBu (2.5 equiv.), THF , $60^{\circ} \mathrm{C}, 4.5 \mathrm{~h}$

(2) imidazole, DMAP, TESCI, DCM, r.t., $12 \mathrm{~h}$<smiles>O=C1N(Cc2ccccc2)c2ccccc2[C@@]1(CBr)OC(F)(F)F</smiles>

$4 a$

A mixture of $\mathrm{Cu}\left(\mathrm{CH}_{3} \mathrm{CN}\right)_{4} \mathrm{PF}_{6}(10 \mathrm{~mol} \%, 0.1 \mathrm{mmol})$, (S)-Monophos $\mathrm{L} 1$ (20 mol \%, $\left.0.2 \mathrm{mmol}\right)$, and LiO'Bu (0.5 equiv, $\left.0.5 \mathrm{mmol}\right)$ in THF (5.0 mL) was stirred for an hour at $25{ }^{\circ} \mathrm{C}$ in a Schlenk tube under an atmosphere of argon. Diborylmethane 2 (1.5 equiv., 1.5 mmol) and LiO'Bu (2.0 equiv., $2.0 \mathrm{mmol}$ ) were weighed into a separate vial and dissolved in THF (10.0 mL) under an atmosphere of argon. An aliquot of the catalyst solution $(5.0 \mathrm{~mL})$ and stir bar was then transferred to the vial containing the diborylmethane and LiOtBu. After 15 minutes at $25^{\circ} \mathrm{C}$, $\mathbf{1 a}(1.0$ equiv, $1.0 \mathrm{mmol}$ in $5.0 \mathrm{~mL} \mathrm{THF})$ was added to the vial via syringe. The reaction was kept at $60{ }^{\circ} \mathrm{C}$ for 4.5 hours and agitated periodically. The reaction was quenched with saturated aqueous solution of $\mathrm{NH}_{4} \mathrm{Cl}(15.0 \mathrm{~mL})$, and the aqueous layer extracted three times with ethyl acetate $(10.0 \mathrm{~mL} \times 3)$. The combined organic extracts were dried over $\mathrm{Na}_{2} \mathrm{SO}_{4}$, filtered, and concentrated in vacuo. The crude reaction mixture was then taken on the next step without purification.

Add imidazole (1.0 equiv, $1.0 \mathrm{mmol}$ ), DMAP (1.0 equiv, $1.0 \mathrm{mmol}$ ) and TESCl (2.0 equiv, $2.0 \mathrm{mmol}$ ) to a solution of the crude hydroxyboronate in DCM $(15.0 \mathrm{~mL})$. Stir the reaction mixture for overnight. The reaction was quenched with saturated aqueous 
solution of $\mathrm{NaHCO}_{3}(20.0 \mathrm{~mL})$, and the aqueous layer extracted three times with $\mathrm{DCM}(10.0 \mathrm{~mL} \times 3)$. The combined organic extracts were dried over $\mathrm{Na}_{2} \mathrm{SO}_{4}$, filtered, and concentrated in vacuo. The product was purified by column chromatography on silica gel to afford $4 \mathrm{a}$ as a colorless oil. (303.7 $\mathrm{mg}, 61 \%$ yield, $88 \%$ ee)

\subsection{Procedure for oxidation of compound $4 a^{4}$}

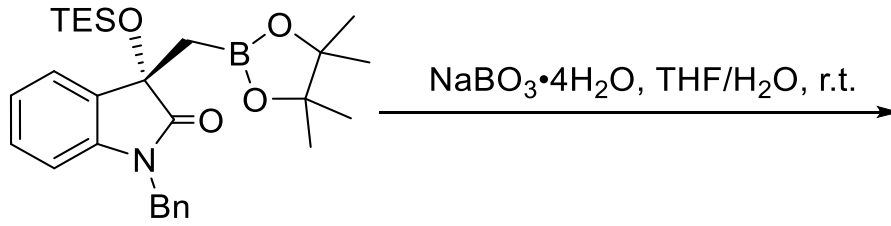

$4 \mathrm{a}$<smiles>N#CO[C@@]1(CO)C(=O)N(Cc2ccccc2)c2ccccc21</smiles>

7

Compound $4 \mathrm{a}(50.0 \mathrm{mg}, 0.1 \mathrm{mmol})$ was combined with sodium perborate tetrahydrate $(77 \mathrm{mg}, 0.5 \mathrm{mmol})$, dissolved in a $1: 1$ mixture of THF and water $(1.0 \mathrm{~mL}, 0.1 \mathrm{M})$ and vigorously stirred for 2 hours at room temperature. The reaction was quenched with saturated aqueous solution of $\mathrm{NH}_{4} \mathrm{Cl}(1.5 \mathrm{~mL})$, and the aqueous layer extracted three times with ethyl acetate $(2 \mathrm{~mL} \times 3)$, dried over $\mathrm{Na}_{2} \mathrm{SO}_{4}$, filtered, and concentrated in vacuo. The product was purified by column chromatography to afford 7 as a white solid. (35.2 $\mathrm{mg}, 92 \%$ yield, $93 \%$ ee)

${ }^{1} \mathrm{H}$ NMR $(400 \mathrm{MHz}$, Chloroform- $d) \delta 7.40(\mathrm{~d}, J=6.8 \mathrm{~Hz}, 1 \mathrm{H}), 7.31-7.22(\mathrm{~m}, 5 \mathrm{H}), 7.18(\mathrm{t}, J=7.7 \mathrm{~Hz}, 1 \mathrm{H}), 7.04(\mathrm{t}, J=7.5 \mathrm{~Hz}, 1 \mathrm{H})$, $5.06(\mathrm{~d}, J=15.8 \mathrm{~Hz}, 1 \mathrm{H}), 4.68(\mathrm{~d}, J=15.8 \mathrm{~Hz}, 1 \mathrm{H}), 4.01-3.93(\mathrm{~m}, 2 \mathrm{H}), 3.83(\mathrm{~s}, 1 \mathrm{H}), 0.82(\mathrm{t}, J=8.0 \mathrm{~Hz}, 9 \mathrm{H}), 0.55-0.46(\mathrm{~m}, 6 \mathrm{H}) .{ }^{13} \mathrm{C}$ NMR $(101 \mathrm{MHz}$, Chloroform-d) $\delta$ 177.2, 143.3, 135.5, 129.6, 129.1, 128.7, 127.5, 127.1, 124.1, 122.9, 109.3, 76.4, 67.1, 43.8, 6.5, 4.2. HRMS (ESI) $\mathrm{m} / \mathrm{z}$ calcd for $\mathrm{C}_{22} \mathrm{H}_{29} \mathrm{NNaO}_{2} \mathrm{Si}[\mathrm{M}+\mathrm{Na}]^{+}:$406.1809, found: 406.1810 . $[\alpha]_{\mathrm{D}}^{25}=-43.89$ (c = 0.20, DCM) HPLC (Chiralpak IC), $n$-hexane/2-propanol = 90/10, flow rate $0.8 \mathrm{~mL} / \mathrm{min}$, detection at $254 \mathrm{~nm}, t_{\mathrm{R}}=12.1 \mathrm{~min}$ (minor), 16.7 min (major).

\subsection{Procedure for alkenylation of compound $4 a^{5}$}<smiles>CC1(C)OB(C[C@]2(OC(F)(F)F)C(=O)N(Cc3ccccc3)c3ccccc32)OC1(C)C</smiles>

4a
1) Vinyl $\mathrm{MgBr}$ (4.0 equiv.), THF, $-78^{\circ} \mathrm{C}, 30 \mathrm{~min}$

2) $\mathrm{I}_{2}$ (4.0 equiv.), $\mathrm{MeOH}$, $-78{ }^{\circ} \mathrm{C}$ to $0{ }^{\circ} \mathrm{C}$<smiles>C=CC[C@]1(OS(=O)(=O)c2ccccc2)C(=O)N(Cc2ccccc2)c2ccccc21</smiles>

8

Vinylmagnesium bromide solution $(0.4 \mathrm{~mL}, 1.0 \mathrm{M}$ in THF, $0.4 \mathrm{mmol})$ was added to a solution of compound $4 \mathrm{aa}(50.0 \mathrm{mg}, 0.1$ $\mathrm{mmol})$ in THF $(1.0 \mathrm{~mL})$ and the reaction mixture was stirred for $30 \mathrm{~min}$ at $-78{ }^{\circ} \mathrm{C}$ under an atmosphere of argon. A solution of iodine $(101.6 \mathrm{mg}, 0.4 \mathrm{mmol})$ in $\mathrm{MeOH}(0.5 \mathrm{~mL})$ was added in a dropwise manner. The mixture was stirred for another $30 \mathrm{~min}$, and allowed to warm to $0{ }^{\circ} \mathrm{C}$. Upon completion of the reaction, the reaction mixture was quenched with sat. aq. $\mathrm{Na}_{2} \mathrm{~S}_{2} \mathrm{O}_{3}(4.0 \mathrm{~mL})$, and extracted with ethyl acetate $(5 \mathrm{~mL} \times 3)$. The combined organic layer was dried over $\mathrm{Na}_{2} \mathrm{SO}_{4}$ and concentrated under reduced pressure. The crude product was purified by column chromatography on silica gel to afford 8 as a colorless oil ( $35.4 \mathrm{mg}, 90 \%$ yield, $91 \%$ ee).

${ }^{1} \mathrm{H}$ NMR $(400 \mathrm{MHz}$, DMSO-d6) $\delta 7.44-7.32(\mathrm{~m}, 7 \mathrm{H}), 7.13(\mathrm{t}, J=7.5 \mathrm{~Hz}, 1 \mathrm{H}), 7.04(\mathrm{~d}, J=7.8 \mathrm{~Hz}, 1 \mathrm{H}), 5.55-5.46(\mathrm{~m}, 1 \mathrm{H}), 5.08$ $-5.01(\mathrm{~m}, 2 \mathrm{H}), 4.83(\mathrm{~d}, J=15.5 \mathrm{~Hz}, 1 \mathrm{H}), 2.80-2.77(\mathrm{~m}, 1 \mathrm{H}), 2.62-2.57(\mathrm{~m}, 1 \mathrm{H}), 0.83(\mathrm{t}, J=7.9 \mathrm{~Hz}, 9 \mathrm{H}), 0.47-0.31(\mathrm{~m}, 6 \mathrm{H}) .{ }^{13} \mathrm{C}$ NMR $(101 \mathrm{MHz}$, DMSO-d6) $\delta 176.4,142.3,136.5,131.6,130.4,130.0,129.0,128.2,128.0,124.6,122.9,120.1,109.8,77.3,44.2$

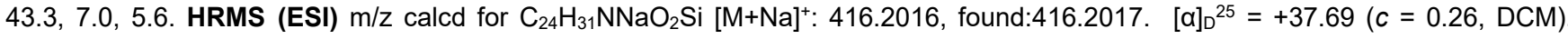
HPLC (CHIRALCEL AD-H), $n$-hexane/2-propanol $=95 / 5$, flow rate $0.8 \mathrm{~mL} / \mathrm{min}$, detection at $254 \mathrm{~nm}, t_{\mathrm{R}}=5.0 \mathrm{~min}$ (minor), $5.4 \mathrm{~min}$ 
(major).

\subsection{Procedure for matteson homologation of compound $4 a^{5}$}
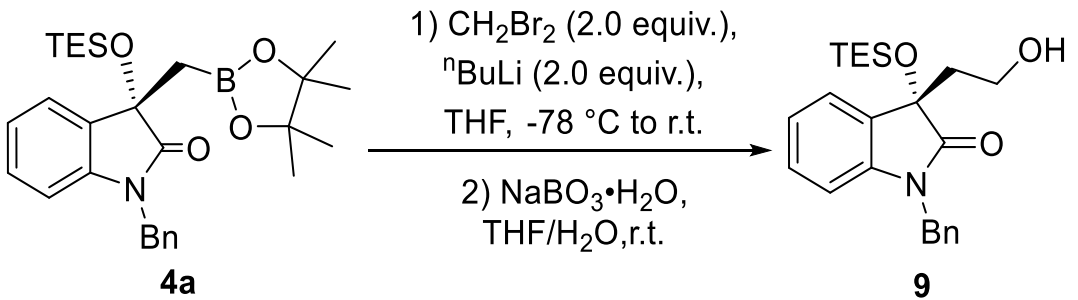

A solution of $4 \mathrm{a}(50.0 \mathrm{mg}, 0.1 \mathrm{mmol})$ and dibromomethane $(43.5 \mathrm{mg}, 0.25 \mathrm{mmol})$ in THF $(1.0 \mathrm{~mL})$ was cooled to $-78{ }^{\circ} \mathrm{C}$ and treated with $n$-BuLi $(0.16 \mathrm{~mL}, 1.6 \mathrm{M}$ in hexanes, $0.25 \mathrm{mmol})$ under an atmosphere of argon. After 30 minutes, the cooling bath was removed and the mixture was stirred at room temperature for overnight. The reaction was quenched with saturated aqueous solution of $\mathrm{NH}_{4} \mathrm{Cl}(1.5 \mathrm{~mL})$, and the aqueous layer extracted three times with ethyl acetate $(2 \mathrm{~mL} \times 3)$, dried over $\mathrm{Na}_{2} \mathrm{SO}_{4}$, filtered, and concentrated in vacuo to get the crude product. And the crude product was combined with sodium perborate tetrahydrate $(77 \mathrm{mg}, 0.5$ $\mathrm{mmol})$, dissolved in a 1:1 mixture of THF and water $(1.0 \mathrm{~mL}, 0.1 \mathrm{M})$ and vigorously stirred for 2 hours at room temperature. The reaction was quenched with saturated aqueous solution of $\mathrm{NH}_{4} \mathrm{Cl}(1.5 \mathrm{~mL})$, and the aqueous layer extracted three times with ethyl acetate $(2 \mathrm{~mL} \times 3)$, dried over $\mathrm{Na}_{2} \mathrm{SO}_{4}$, filtered, and concentrated in vacuo. The product was purified by column chromatography to afford 9 as a colorless oil ( $27.0 \mathrm{mg}, 68 \%$ yield, $94 \%$ ee).

${ }^{1} \mathrm{H}$ NMR $(400 \mathrm{MHz}$, Chloroform-d) $\delta 7.35-7.20(\mathrm{~m}, 7 \mathrm{H}), 7.06(\mathrm{t}, J=7.5 \mathrm{~Hz}, 1 \mathrm{H}), 6.77$ (d, $J=7.8 \mathrm{~Hz}, 1 \mathrm{H}), 5.05(\mathrm{~d}, J=15.4 \mathrm{~Hz}$, $1 \mathrm{H}), 4.68(\mathrm{~d}, J=15.4 \mathrm{~Hz}, 1 \mathrm{H}), 4.17-4.11(\mathrm{~m}, 1 \mathrm{H}), 3.85-3.81(\mathrm{~m}, 1 \mathrm{H}), 3.23(\mathrm{~s}, 1 \mathrm{H}), 2.13-2.00(\mathrm{~m}, 2 \mathrm{H}), 0.82(\mathrm{t}, J=7.9 \mathrm{~Hz}, 9 \mathrm{H})$, $0.44-0.32(\mathrm{~m}, 6 \mathrm{H}) .{ }^{13} \mathrm{C}$ NMR $(101 \mathrm{MHz}$, Chloroform-d) $\delta 178.2,141.5,135.4,131.6,129.59,128.8,127.8,127.7,124.0,123.1,109.4$, 58.6, 44.0, 42.0, 6.6, 5.5. HRMS (ESI) $\mathrm{m} / \mathrm{z}$ calcd for $\mathrm{C}_{22} \mathrm{H}_{29} \mathrm{NnaO}_{2} \mathrm{Si}[\mathrm{M}+\mathrm{Na}]^{+}:$420.1965, found: $420.1968 .[\alpha]_{D}{ }^{25}=-44.17(c=0.19$, DCM) HPLC (Chiralpak IC), $n$-hexane/2-propanol = 90/10, flow rate $0.8 \mathrm{~mL} / \mathrm{min}$, detection at $254 \mathrm{~nm}, t_{\mathrm{R}}=10.2 \mathrm{~min}(\mathrm{minor}), 13.3 \mathrm{~min}$ (major).

\subsection{Procedure for heterocyclization of compound $4 a^{6}$}<smiles>CC1(C)OB(C[C@]2(OS(F)(F)F)C(=O)N(Cc3ccccc3)c3ccccc32)OC1(C)C</smiles>

$4 a$
1) Thiophene (1.2 equiv.), ${ }^{\mathrm{B}} \mathrm{BuLi}(1.2$ equiv.), THF, $-78^{\circ} \mathrm{C}$ to r.t.

2) NBS (1.2 equiv.), THF, $-78^{\circ} \mathrm{C}$<smiles>C=CCC</smiles><smiles>O=C1N(Cc2ccccc2)c2ccccc2[C@@]1(Cc1cccs1)OCc1ccccc1</smiles>

10

A solution of thiophene $(20.2 \mathrm{mg}, 0.24 \mathrm{mmol})$ in THF $(1.0 \mathrm{~mL})$ was cooled to $-78^{\circ} \mathrm{C}$ and treated with $n$-BuLi $(0.15 \mathrm{~mL}, 1.6 \mathrm{M}$ in hexanes, $0.24 \mathrm{mmol}$ ) under an atmosphere of argon. The cooling bath was removed and the mixture was stirred at room temperature for 1 hour. The mixture was cooled to $-78^{\circ} \mathrm{C}$ and a solution of compound $4 \mathrm{a}$ (100.0 $\left.\mathrm{mg}, 0.2 \mathrm{mmol}\right)$ in THF (1.0 mL) was added. The mixture was stirred at $-78{ }^{\circ} \mathrm{C}$ for 1 hour and then, a solution of NBS $(42.7 \mathrm{mg}, 0.24 \mathrm{mmol})$ in THF $(1.0 \mathrm{~mL})$ was added. After 1 hour at $-78{ }^{\circ} \mathrm{C}$, sat. aq. $\mathrm{Na}_{2} \mathrm{~S}_{2} \mathrm{O}_{3}(4.0 \mathrm{~mL})$ was added and the reaction mixture was allowed to warm to room temperature. The mixture was extracted with ethyl acetate $(5 \mathrm{~mL} \times 3)$. The combined organic layer was dried over $\mathrm{Na}_{2} \mathrm{SO}_{4}$ and concentrated under reduced pressure. The crude product was purified by column chromatography on silica gel to afforded 10 as a yellow oil ( $38.6 \mathrm{mg}, 43 \%$ yield, $93 \%$ ee).

${ }^{1} \mathrm{H}$ NMR $(400 \mathrm{MHz}$, DMSO-d6) $\delta 7.29-7.20(\mathrm{~m}, 6 \mathrm{H}), 7.05(\mathrm{t}, \mathrm{J}=7.4 \mathrm{~Hz}, 1 \mathrm{H}), 6.96-6.94(\mathrm{~m}, 2 \mathrm{H}), 6.86-6.84(\mathrm{~m}, 1 \mathrm{H}), 6.76(\mathrm{~d}$, 
$J=7.8 \mathrm{~Hz}, 1 \mathrm{H}), 6.60(\mathrm{~d}, J=3.4 \mathrm{~Hz}, 1 \mathrm{H}), 4.92(\mathrm{~d}, J=15.7 \mathrm{~Hz}, 1 \mathrm{H}), 4.62(\mathrm{~d}, J=15.8 \mathrm{~Hz}, 1 \mathrm{H}), 3.50(\mathrm{~d}, J=13.8 \mathrm{~Hz}, 1 \mathrm{H}), 3.35(\mathrm{~d}, J=$ $12.6 \mathrm{~Hz}, 1 \mathrm{H}), 0.78(\mathrm{t}, J=7.9 \mathrm{~Hz}, 9 \mathrm{H}), 0.43-0.25(\mathrm{~m}, 6 \mathrm{H}) .{ }^{13} \mathrm{C}$ NMR $(101 \mathrm{MHz}, \mathrm{DMSO}-d 6) \delta 176.1,142.9,136.1,136.1,130.4$, 129.8, 128.9, 128.3, 127.7, 127.6, 126.7, 126.0, 124.9, 122.9, 109.8, 77.9, 43.3, 39.9, 7.0, 5.5. HRMS (ESI) m/z calcd for

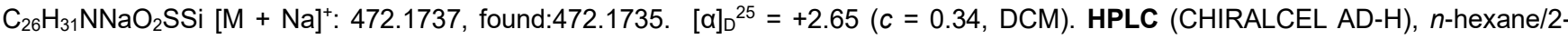
propanol $=95 / 5$, flow rate $0.8 \mathrm{~mL} / \mathrm{min}$, detection at $254 \mathrm{~nm}, t_{\mathrm{R}}=6.2 \mathrm{~min}$ (minor), $7.9 \mathrm{~min}$ (major).

\subsection{Procedure for oxidation of hydroxyboronate ${ }^{4}$}<smiles>CN1C(=O)C(=O)c2ccccc21</smiles>

$1 \mathrm{a}^{\mathrm{Bn}}$

$$
\text { Bpin } \widehat{B p i n}
$$

2

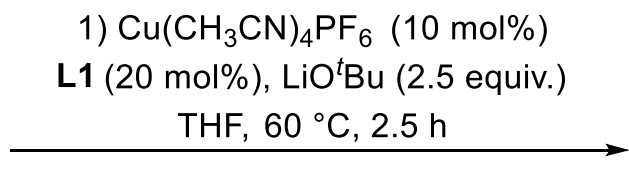

2) $\mathrm{NaBO}_{3} \cdot 4 \mathrm{H}_{2} \mathrm{O}, \mathrm{THF} / \mathrm{H}_{2} \mathrm{O}, 2 \mathrm{~h}$<smiles>O=C1N(Br)c2ccccc2[C@@]1(O)CO</smiles>

A mixture of $\mathrm{Cu}\left(\mathrm{CH}_{3} \mathrm{CN}\right)_{4} \mathrm{PF}_{6}(3.6 \mathrm{mg}, 10 \mathrm{~mol} \%)$, (S)-Monophos L1 (7.4 mg, $20 \mathrm{~mol} \%$ ), and LiO'Bu (4.0 mg, $\left.0.05 \mathrm{mmol}\right)$ in THF $(0.5 \mathrm{~mL})$ was stirred for 45 minutes at $25{ }^{\circ} \mathrm{C}$ in a Schlenk tube under an atmosphere of argon. Diborylmethane 2 (40.2 mg, 0.15 $\mathrm{mmol}$ ) and LiO'Bu (16.0 mg, $0.2 \mathrm{mmol}$ ) were weighed into a separate vial and dissolved in THF (1.0 mL) under an atmosphere of argon. An aliquot of the catalyst solution $(0.5 \mathrm{~mL})$ and stir bar was then transferred to the vial containing the diborylmethane and LiO'Bu. After 15 minutes at $25^{\circ} \mathrm{C}, 1 \mathrm{a}(23.7 \mathrm{mg}, 0.1 \mathrm{mmol}$ in $0.5 \mathrm{~mL}$ THF) was added to the vial via syringe. The reaction was kept at $60{ }^{\circ} \mathrm{C}$ for 2.5 hours and agitated periodically. The reaction was quenched with saturated aqueous solution of $\mathrm{NH}_{4} \mathrm{Cl}(1.5 \mathrm{~mL})$, and the aqueous layer extracted three times with ethyl acetate $(2 \mathrm{~mL} \times 3)$. The combined organic extracts were dried over $\mathrm{Na}_{2} \mathrm{SO}_{4}$, filtered, and concentrated in vacuo. The crude reaction mixture was then taken on the next step without purification.

The crude hydroxyboronate was combined with sodium perborate tetrahydrate $(77 \mathrm{mg}, 0.5 \mathrm{mmol})$, dissolved in a $1: 1$ mixture of THF and water $(1.0 \mathrm{~mL}, 0.1 \mathrm{M})$ and vigorously stirred for 2 hours at room temperature. The reaction was quenched with saturated aqueous solution of $\mathrm{NH}_{4} \mathrm{Cl}(1.5 \mathrm{~mL})$, and the aqueous layer extracted three times with ethyl acetate $(2 \mathrm{~mL} \times 3)$, dried over $\mathrm{Na}_{2} \mathrm{SO}_{4}$, filtered, and concentrated in vacuo. The product was purified by column chromatography on silica gel to afford $\mathbf{1 1}$ as a white solid(15.6 mg, 58\% yield over two steps, 93\% ee).

${ }^{1} \mathrm{H}$ NMR $(400 \mathrm{MHz}$, DMSO-d6) $\delta 7.38-7.17(\mathrm{~m}, 7 \mathrm{H}), 7.03(\mathrm{t}, J=7.4 \mathrm{~Hz}, 1 \mathrm{H}), 6.76(\mathrm{~d}, J=7.8 \mathrm{~Hz}, 1 \mathrm{H}), 6.13(\mathrm{~s}, 1 \mathrm{H}), 4.99-4.94$ $(\mathrm{m}, 2 \mathrm{H}), 4.80(\mathrm{~d}, J=16.0 \mathrm{~Hz}, 1 \mathrm{H}), 3.79-3.73(\mathrm{~m}, 2 \mathrm{H}) .{ }^{13} \mathrm{C}$ NMR $(101 \mathrm{MHz}$, DMSO-d6) $\delta 177.7,143.7,136.7,131.3,129.31,128.9$, 127.7, 127.6, 124.6, 122.6, 109.2, 76.6, 65.9, 42.9. HRMS (ESI) m/z calcd for $\mathrm{C}_{24} \mathrm{H}_{31} \mathrm{NNaO}_{2} \mathrm{Si}[\mathrm{M}+\mathrm{Na}]^{+}: 292.0944$, found: 292.0937. $[\alpha]_{D}{ }^{25}=-106.67(c=0.06$, DCM). HPLC (CHIRALCEL AD-H), $n$-hexane/2-propanol $=80 / 20$, flow rate $0.8 \mathrm{~mL} / \mathrm{min}$, detection at 254 $\mathrm{nm}, t_{\mathrm{R}}=28.3 \mathrm{~min}$ (major), $30.3 \mathrm{~min}$ (minor).

\subsection{Procedure for oxidation of substituted hydroxyboronate ${ }^{4}$}<smiles>O=C1C(=O)N(Cc2ccccc2)c2ccccc21</smiles>

1a<smiles>Brc1ccccc1</smiles>

$5 c$
1) $\mathrm{Cu}\left(\mathrm{CH}_{3} \mathrm{CN}\right)_{4} \mathrm{PF}_{6}(10 \mathrm{~mol} \%)$ L1 (20 mol\%), LiO ${ }^{t} \mathrm{Bu}$ (2.5 equiv.) $\mathrm{THF}, 60^{\circ} \mathrm{C}, 8 \mathrm{~h}$

2) $\mathrm{NaBO}_{3} \cdot 4 \mathrm{H}_{2} \mathrm{O}, \mathrm{THF} / \mathrm{H}_{2} \mathrm{O}$, r.t. $2 \mathrm{~h}$<smiles>O=C1N(Cc2ccccc2)c2ccccc2C1(O)CCc1ccccc1</smiles>

12

A mixture of $\mathrm{Cu}\left(\mathrm{CH}_{3} \mathrm{CN}\right)_{4} \mathrm{PF}_{6}$ (3.6 mg, $\left.10 \mathrm{~mol} \%\right)$, (S)-Monophos L1 (7.4 mg, $20 \mathrm{~mol} \%$ ), and LiO'Bu (4.0 mg, $\left.0.05 \mathrm{mmol}\right)$ in THF $(0.5 \mathrm{~mL})$ was stirred for 45 minutes at $25{ }^{\circ} \mathrm{C}$ in a Schlenk tube under an atmosphere of argon. $5 \mathrm{c}(55.8 \mathrm{mg}, 0.15 \mathrm{mmol})$ and LiO'Bu 
(16.0 mg, $0.2 \mathrm{mmol}$ ) were weighed into a separate vial and dissolved in THF (1.0 mL) under an atmosphere of argon. An aliquot of the catalyst solution $(0.5 \mathrm{~mL})$ and stir bar was then transferred to the vial containing the diborylmethane and LiOtBu. After 15 minutes at $25^{\circ} \mathrm{C}, 1 \mathrm{a}\left(23.7 \mathrm{mg}, 0.1 \mathrm{mmol}\right.$ in $0.5 \mathrm{~mL}$ THF) was added to the vial via syringe. The reaction was kept at $60{ }^{\circ} \mathrm{C}$ for 8 hours and agitated periodically. The reaction was quenched with saturated aqueous solution of $\mathrm{NH}_{4} \mathrm{Cl}(1.5 \mathrm{~mL})$, and the aqueous layer extracted three times with ethyl acetate $(2 \mathrm{~mL} \times 3)$. The combined organic extracts were dried over $\mathrm{Na}_{2} \mathrm{SO}_{4}$, filtered, and concentrated in vacuo. The crude reaction mixture was then taken on the next step without purification.

The crude hydroxyboronate was combined with sodium perborate tetrahydrate $(77 \mathrm{mg}, 0.5 \mathrm{mmol})$, dissolved in a 1:1 mixture of THF and water $(1.0 \mathrm{~mL}, 0.1 \mathrm{M})$ and vigorously stirred for 2 hours at room temperature. The reaction was quenched with saturated aqueous solution of $\mathrm{NH}_{4} \mathrm{Cl}(1.5 \mathrm{~mL})$, and the aqueous layer extracted three times with ethyl acetate $(3 \mathrm{~mL} \times 3)$, dried over $\mathrm{Na}_{2} \mathrm{SO}_{4}$, filtered, and concentrated in vacuo. The product was purified by column chromatography on silica gel to afford 12 as a white solid(16.0 mg, 43\% yield over two steps, , > 20:1 d.r., 92\% ee).

${ }^{1} \mathrm{H}$ NMR (400 MHz, DMSO-d6) $\delta 7.39-7.37(\mathrm{~m}, 2 \mathrm{H}), 7.32-7.16(\mathrm{~m}, 10 \mathrm{H}), 6.96(\mathrm{t}, \mathrm{J}=7.4 \mathrm{~Hz}, 1 \mathrm{H}), 6.70(\mathrm{~d}, \mathrm{~J}=7.7 \mathrm{~Hz}, 1 \mathrm{H}), 5.01$ $(\mathrm{d}, \mathrm{J}=16.1 \mathrm{~Hz}, 1 \mathrm{H}), 4.71(\mathrm{~d}, \mathrm{~J}=16.1 \mathrm{~Hz}, 1 \mathrm{H}), 3.82(\mathrm{~d}, \mathrm{~J}=9.3 \mathrm{~Hz}, 1 \mathrm{H}), 2.85-2.78(\mathrm{~m}, 1 \mathrm{H}), 2.58-2.54(\mathrm{~m}, 1 \mathrm{H}), 2.31-2.22(\mathrm{~m}, 1 \mathrm{H})$, $1.77-1.67$ (m, 1H). ${ }^{13} \mathrm{C}$ NMR (101 MHz, DMSO-d6) $\delta$ 178.7, 144.2, 142.8, 136.8, 130.0, 129.2, 128.9, 128.9, 128.8, 127.6, 126.1, 125.8, 122.1, 109.1, 78.6, 73.9, 42.9, 32.9, 32.4. HRMS (ESI) m/z calcd for $\mathrm{C}_{24} \mathrm{H}_{31} \mathrm{NNaO}_{2} \mathrm{Si}[\mathrm{M}+\mathrm{Na}]^{+}: 396.1570$, found:396.1571. $[\alpha]_{D}^{25}=+18.33(c=0.10$, DCM). HPLC (CHIRALCEL AD-H), $n$-hexane/2-propanol = 80/20, flow rate $0.8 \mathrm{~mL} / \mathrm{min}$, detection at $254 \mathrm{~nm}$, $t_{\mathrm{R}}=14.0 \mathrm{~min}$ (major), $15.0 \mathrm{~min}$ (minor). 


\section{References}

1. Buxton. C. S.; Blakemore. D. C.; Bower. J. F. Reductive Coupling of Acrylates with Ketones and Ketimines by a NickelCatalyzed Transfer-Hydrogenative Strategy. Angew. Chem., Int. Ed. 2017, 56, 13824-13828.

2. He, T. -T; Peng, L.; Li, S.; Hu, F.-L; Xie, C.-D; Huang, S.-L; Jia, S.-Q; Qin, W.L; Yan, H.-L. Chiral Naphthyl-C2-Indole as Scaffold for Phosphine Organocatalysis: Application in Asymmetric Formal [4 + 2] Cycloaddition Reactions. Org. Lett. 2020, 22, 6966-6971

3. Joannou, M. V.; Moyer, B. S.; Goldfogel, M. J.; Meek, S. J. Silver(I)-Catalyzed Diastereoselective Synthesis of Anti-1,2Hydroxyboronates. Angew. Chem., Int. Ed. 2015, 54, 14141-14145.

4. Murray, S. A.; Green, J. C.; Tailor, S. B.; Meek, S. J. Enantio- and Diastereoselective 1,2-Additions to alpha-Ketoesters with Diborylmethane and Substituted 1,1-Diborylalkanes. Angew. Chem., Int. Ed. 2016, 55, 9065-9069.

5. Sonawane, R. P.; Jheengut, V.; Rabalakos, C.; Larouche-Gauthier, R.; Scott, H. K.; Aggarwal, V. K. Enantioselective Construction of Quaternary Stereogenic Centers from Tertiary Boronic esters: methodology and applications. Angew. Chem., Int. Ed. 2011, 50, 3760-3763.

6. Bonet, A.; Odachowski, M.; Leonori, D.; Essafi, S.; Aggarwal, V. K. Enantiospecific sp(2)-sp(3) Coupling of Secondary and Tertiary Boronic Esters. Nat. Chem. 2014, 6, 584-589. 
4. Spectral Data

${ }^{1} \mathrm{H}$ NMR Spectrum of Compound $4 \mathrm{a}(400 \mathrm{MHz}$, DMSO-d6)

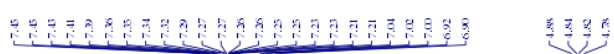

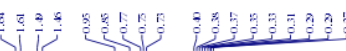

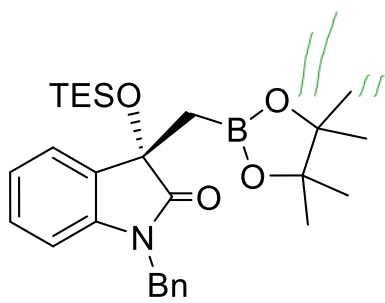

$4 a$

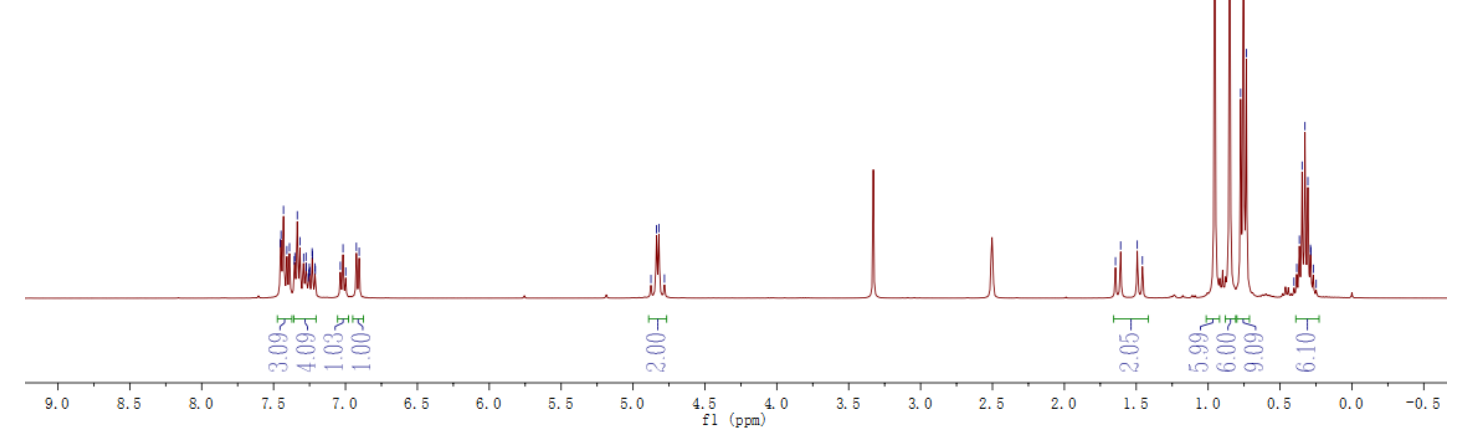

${ }^{13} \mathrm{C}$ NMR Spectrum of Compound 4a (101 MHz, DMSO-d6)

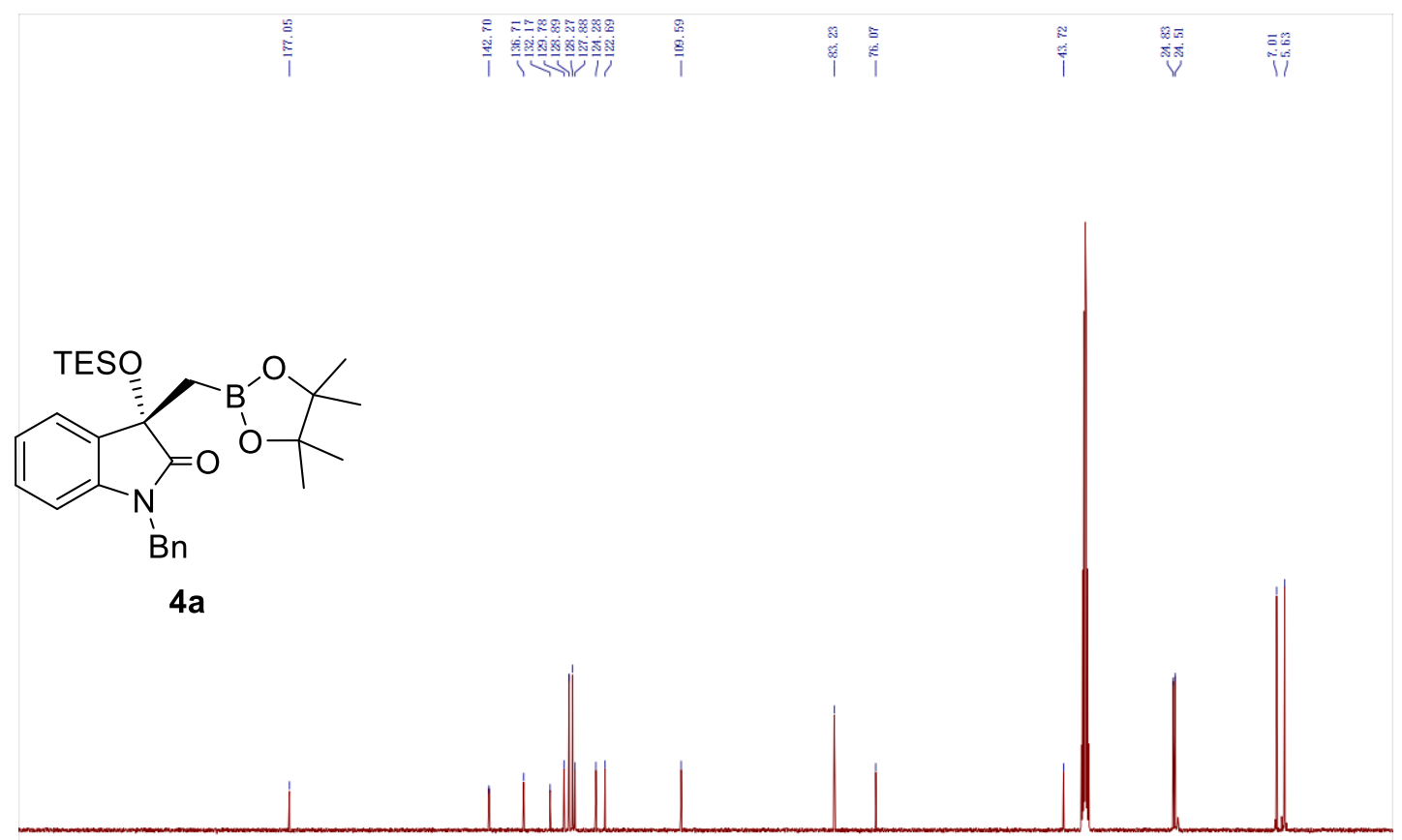

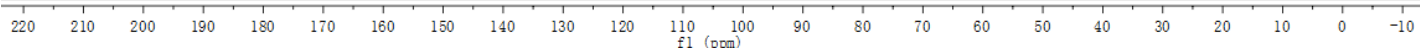


${ }^{11} \mathrm{~B}$ NMR Spectrum of Compound 4a (193 $\left.\mathrm{MHz}, \mathrm{CDCl}_{3}\right)$<smiles>COC1(CB2OC(C)(C)C(C)(C)O2)C(=O)Nc2ccccc21</smiles>

$4 a$

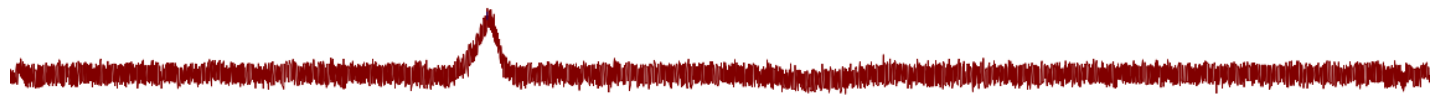


${ }^{1} \mathrm{H}$ NMR Spectrum of Compound $4 \mathrm{~b}\left(400 \mathrm{MHz}, \mathrm{CDCl}_{3}\right)$

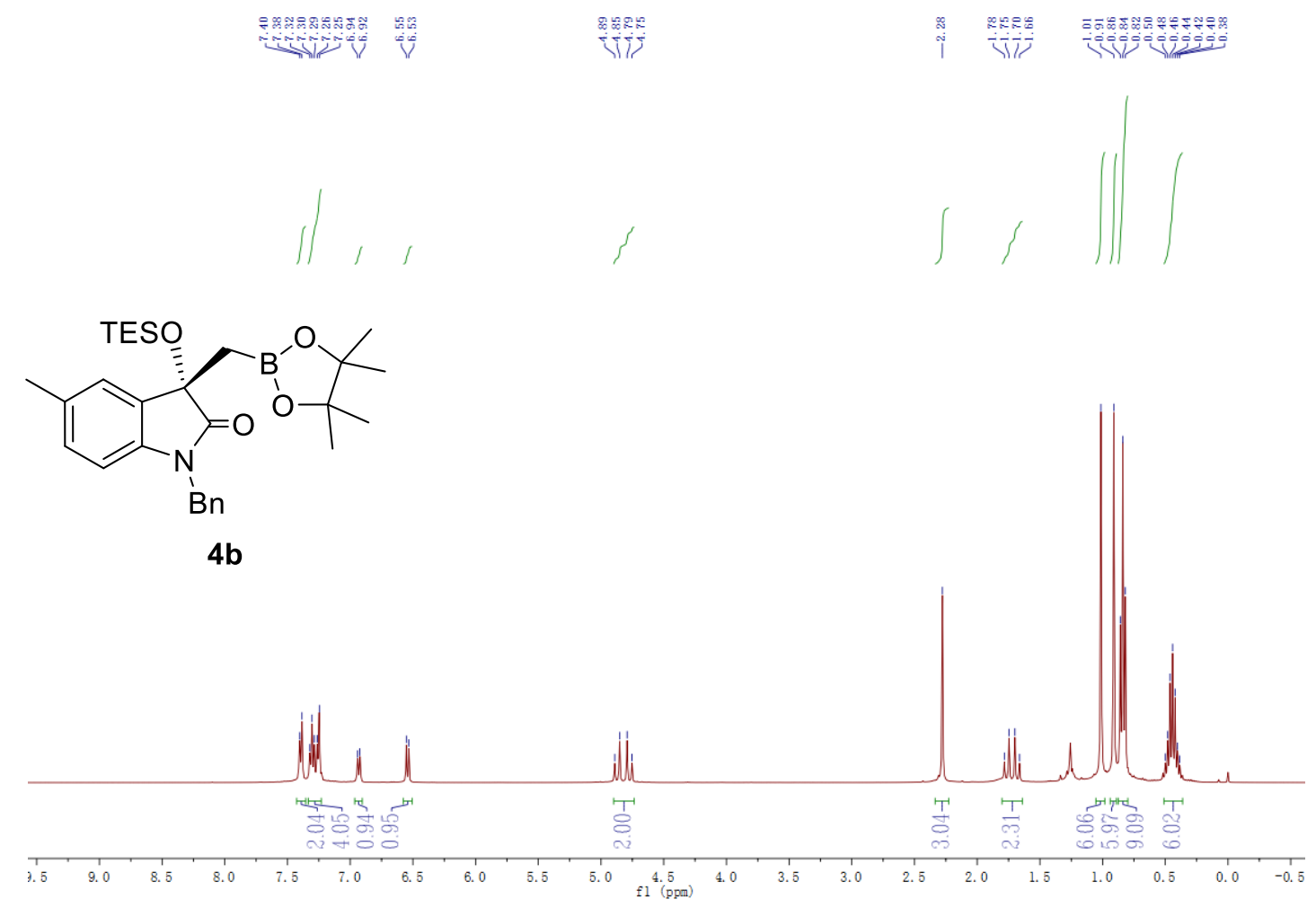

${ }^{13} \mathrm{C}$ NMR Spectrum of Compound $4 \mathrm{~b}\left(151 \mathrm{MHz}, \mathrm{CDCl}_{3}\right)$
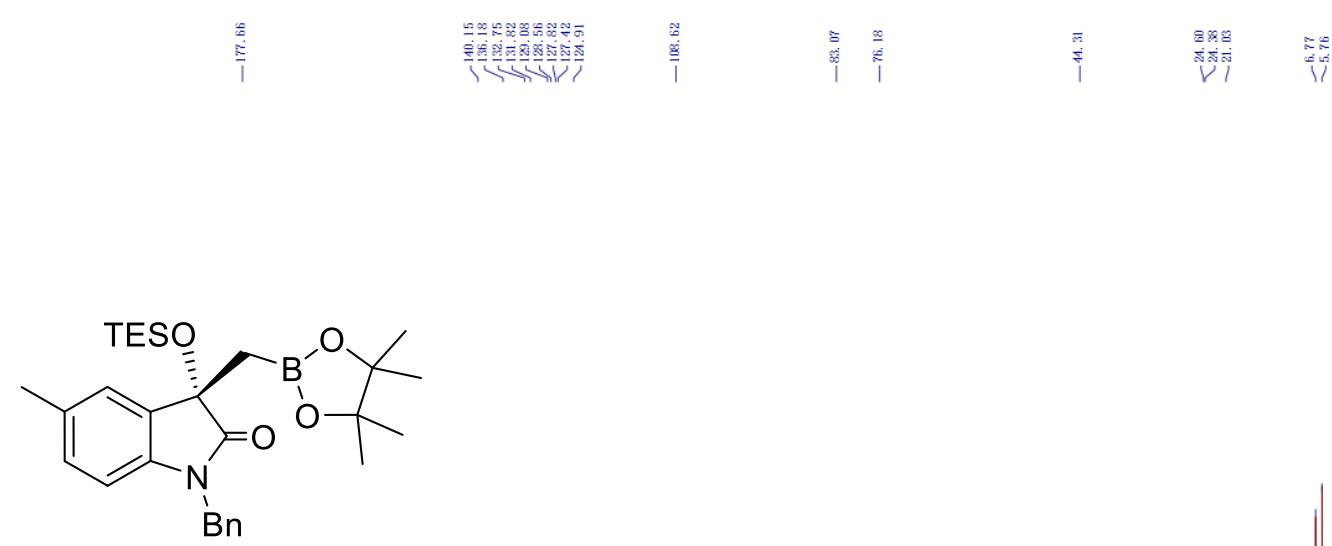

4b

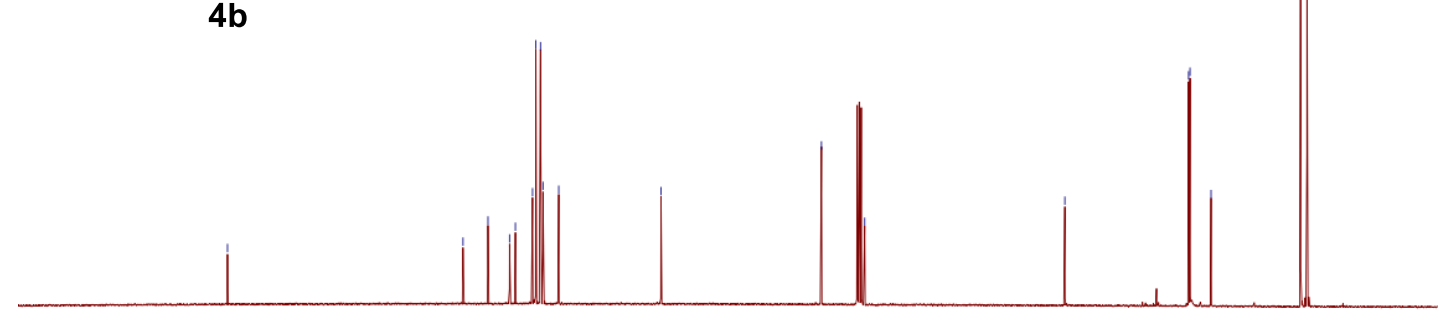

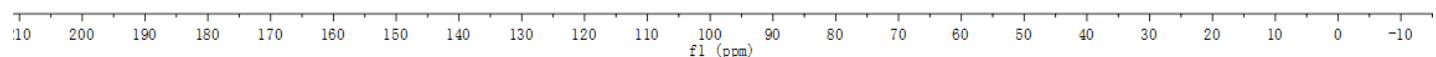


${ }^{11} \mathrm{~B}$ NMR Spectrum of Compound 4b (193 MHz, $\left.\mathrm{CDCl}_{3}\right)$

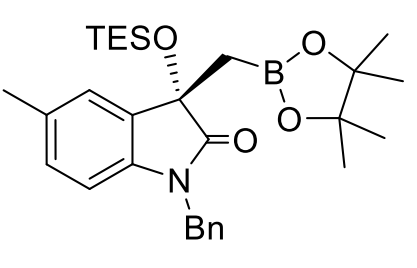

4b

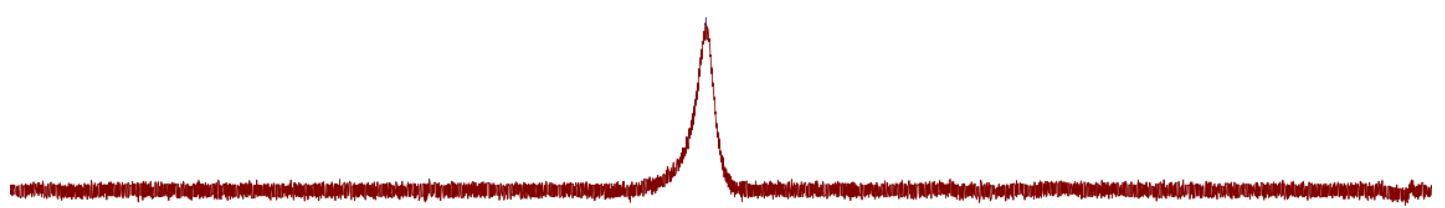


${ }^{1} \mathrm{H}$ NMR Spectrum of Compound 4c (400 MHz, DMSO-d6)

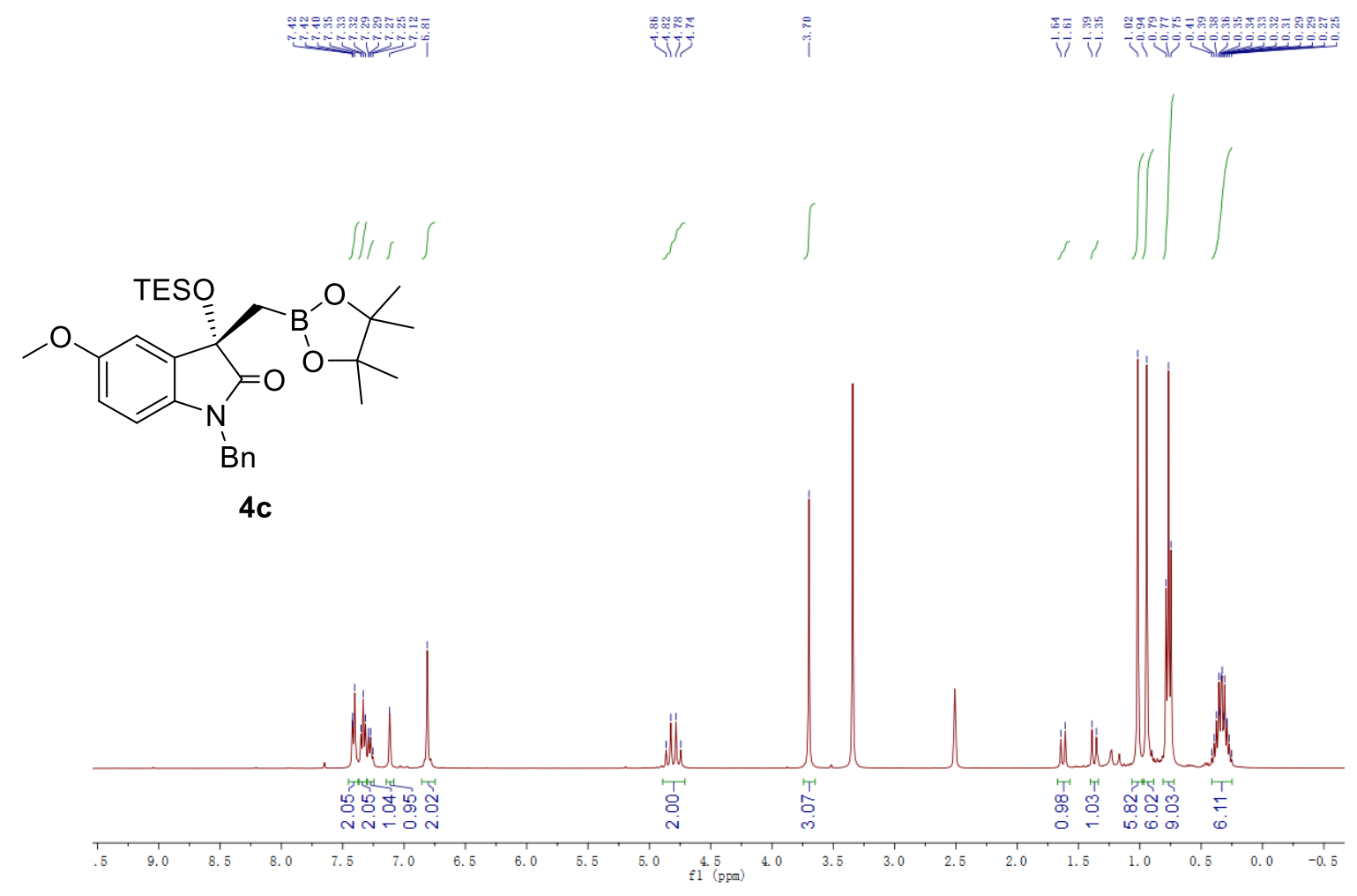

${ }^{13} \mathrm{C}$ NMR Spectrum of Compound 4c (151 MHz, DMSO-d6)
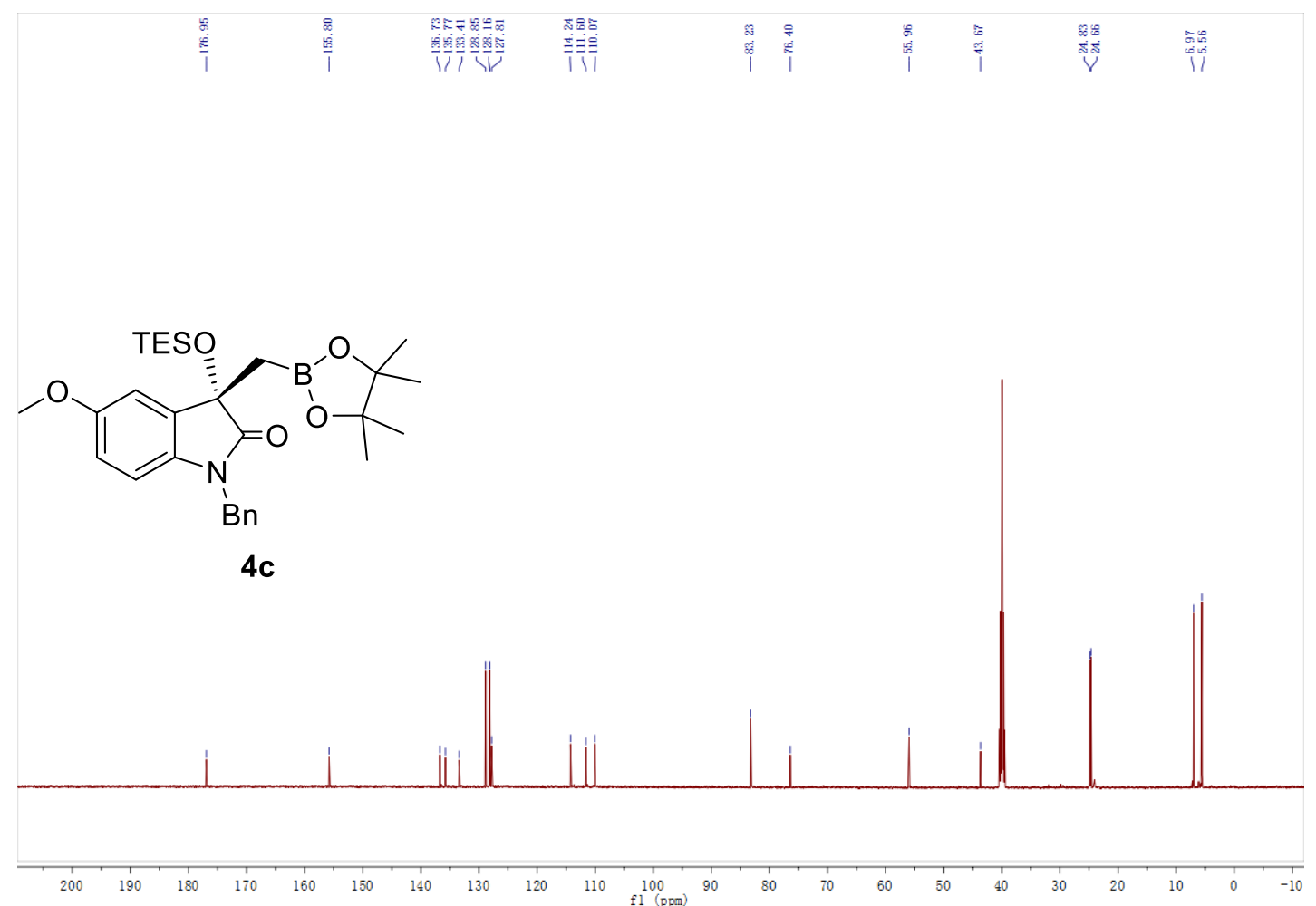
11B NMR Spectrum of Compound 4c (193 MHz, DMSO-d6)<smiles>COc1ccc2c(c1)[C@](CB1OC(C)(C)C(C)(C)O1)(OC(C)(C)C)C(=O)N2Cc1ccccc1</smiles>

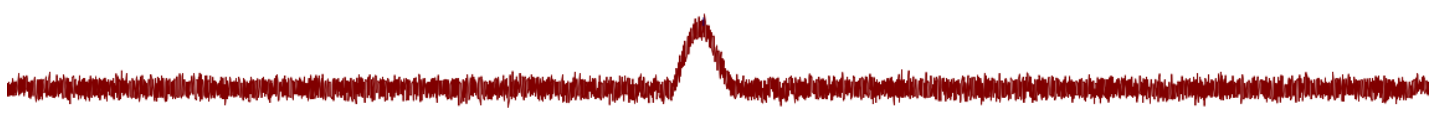


${ }^{1} \mathrm{H}$ NMR Spectrum of Compound $4 \mathrm{~d}\left(400 \mathrm{MHz}, \mathrm{CDCl}_{3}\right)$
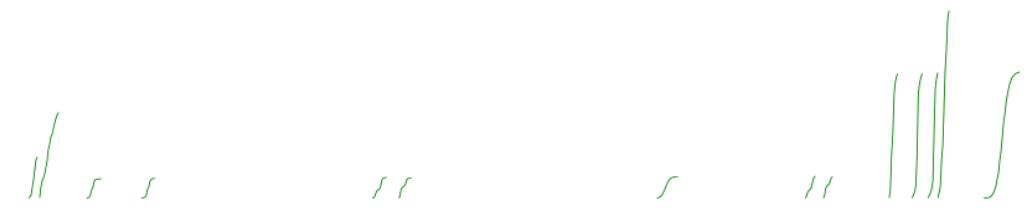<smiles>CC(C)c1ccc2c(c1)[C@](CB1OC(C)(C)C(C)(C)O1)(OC(F)(F)F)C(=O)N2Cc1ccccc1</smiles>

4d

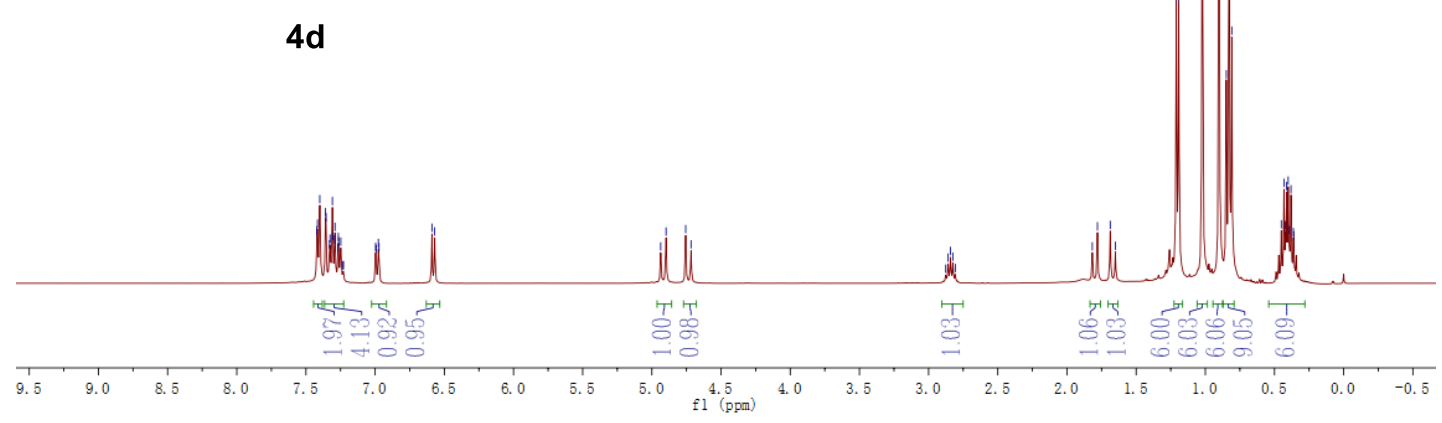

${ }^{13} \mathrm{C}$ NMR Spectrum of Compound $4 \mathrm{~d}\left(101 \mathrm{MHz}, \mathrm{CDCl}_{3}\right)$

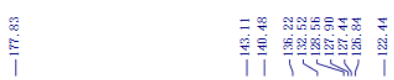<smiles>CC(C)c1ccc2c(c1)[C@](CB1OC(C)(C)C(C)(C)O1)(OS(F)(F)F)C(=O)N2Cc1ccccc1</smiles>
4d
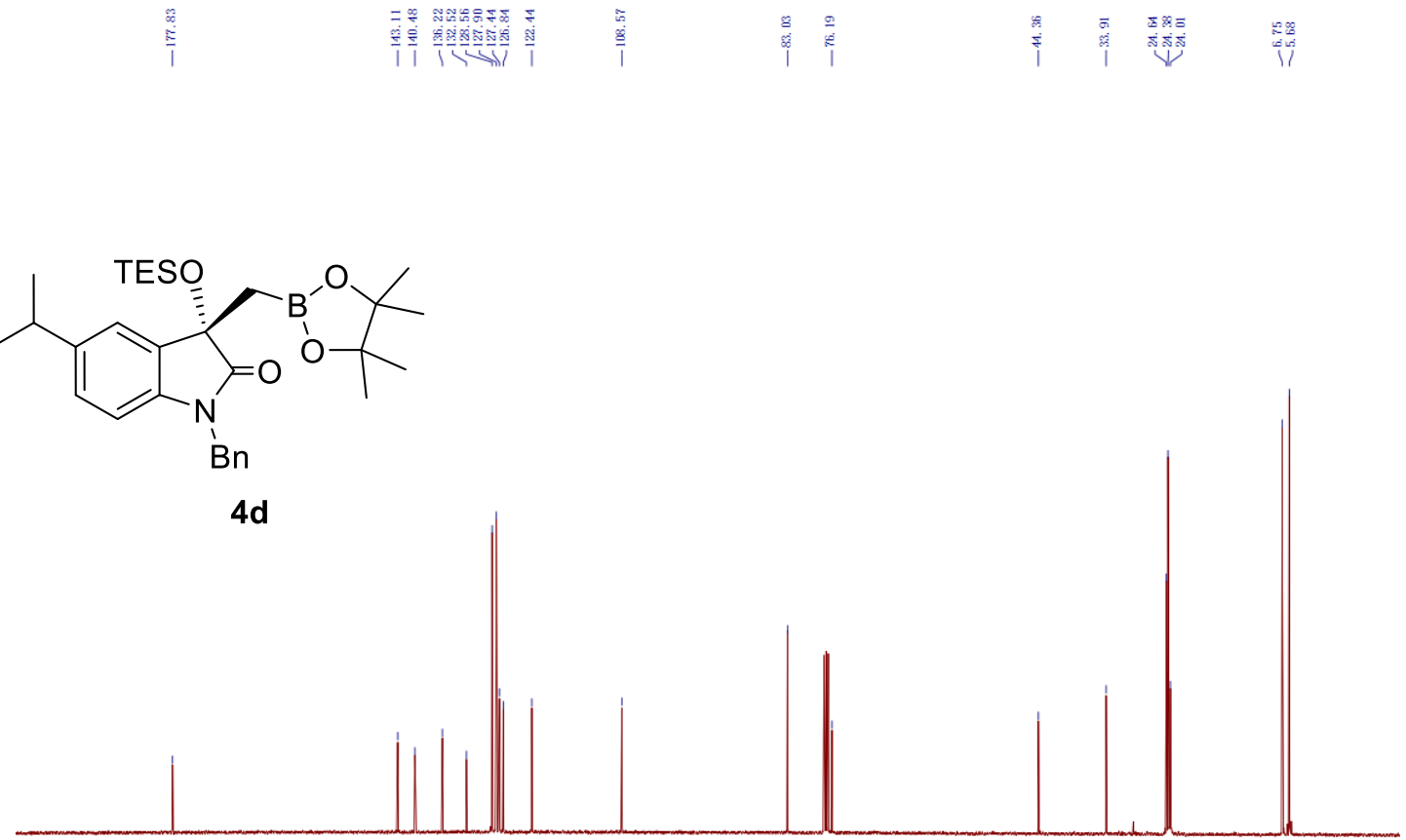

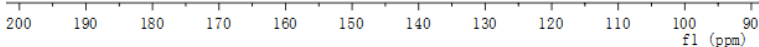


${ }^{11} \mathrm{~B}$ NMR Spectrum of Compound 4d (193 MHz, $\mathrm{CDCl}_{3}$ )
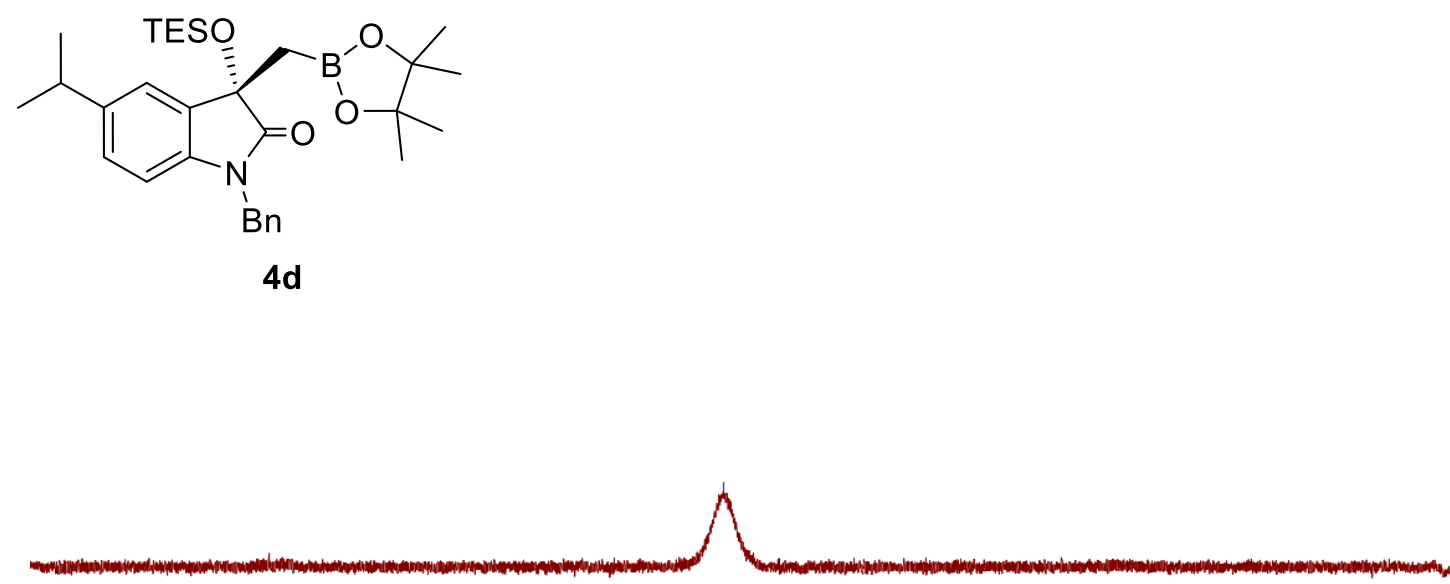
${ }^{1} \mathrm{H}$ NMR Spectrum of Compound $4 \mathrm{e}(400 \mathrm{MHz}$, DMSO-d6)

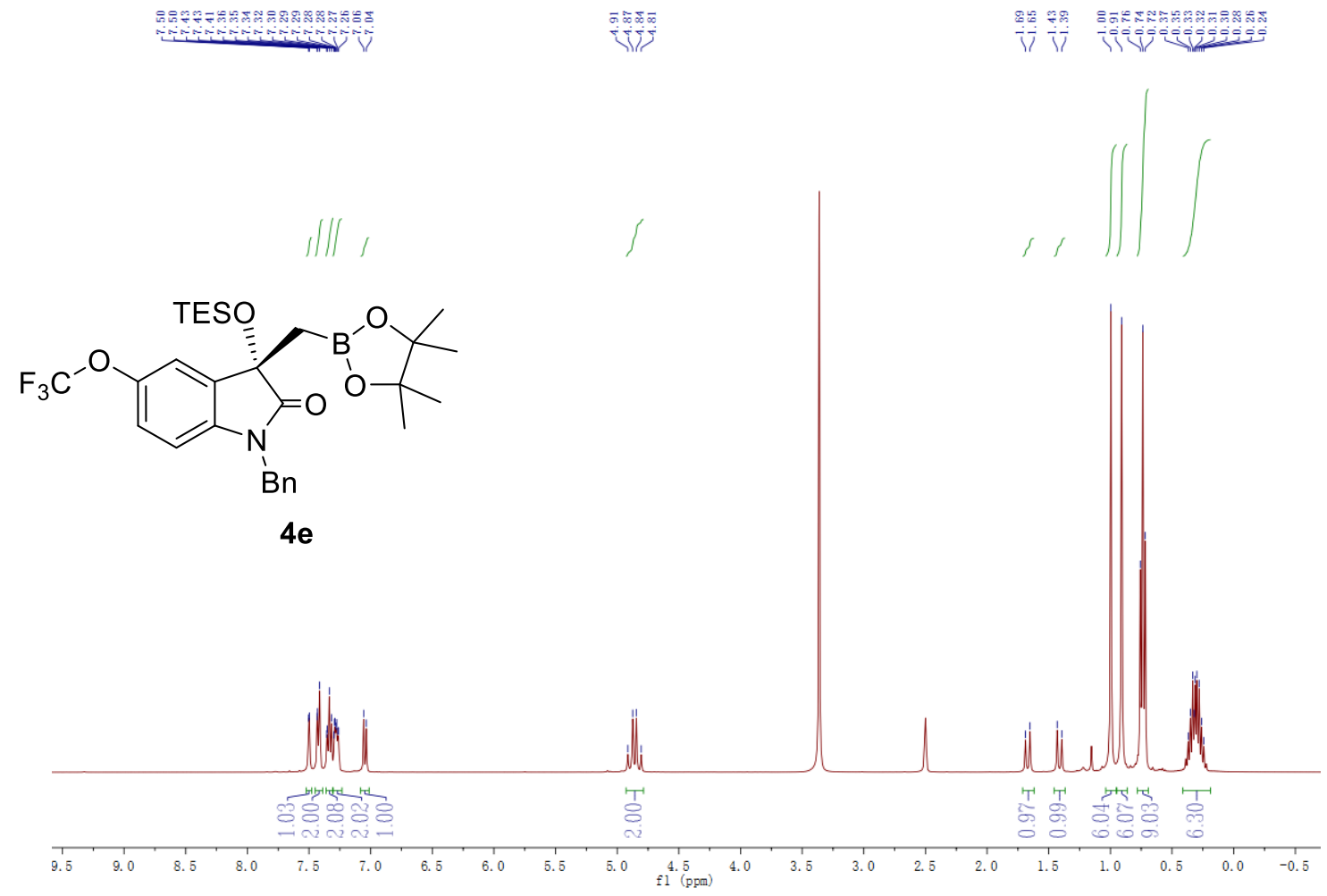

${ }^{13} \mathrm{C}$ NMR Spectrum of Compound 4e (101 MHz, DMSO-d6)
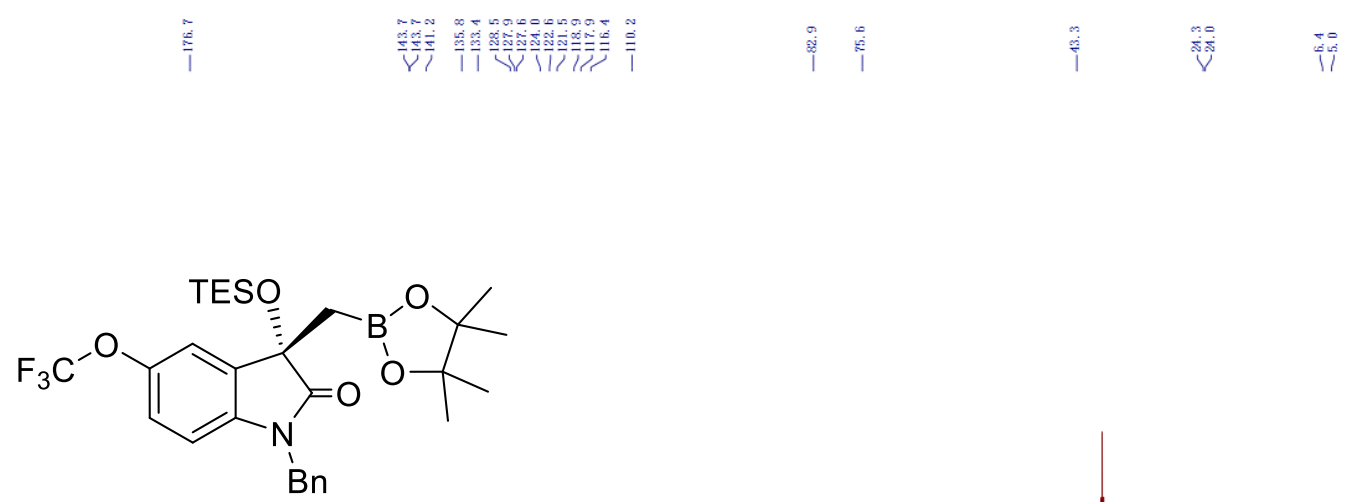

$4 e$
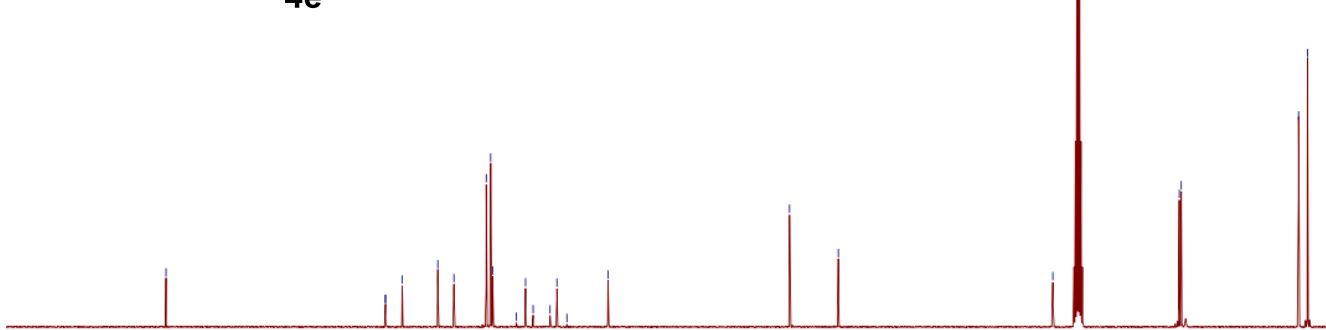
${ }^{11} \mathrm{~B}$ NMR Spectrum of Compound 4e (193 MHz, $\left.\mathrm{CDCl}_{3}\right)$<smiles>CC1(C)OB(C[C@]2(OC(F)(F)F)C(=O)N(Cc3ccccc3)c3ccc(OC(F)(F)F)cc32)OC1(C)C</smiles>
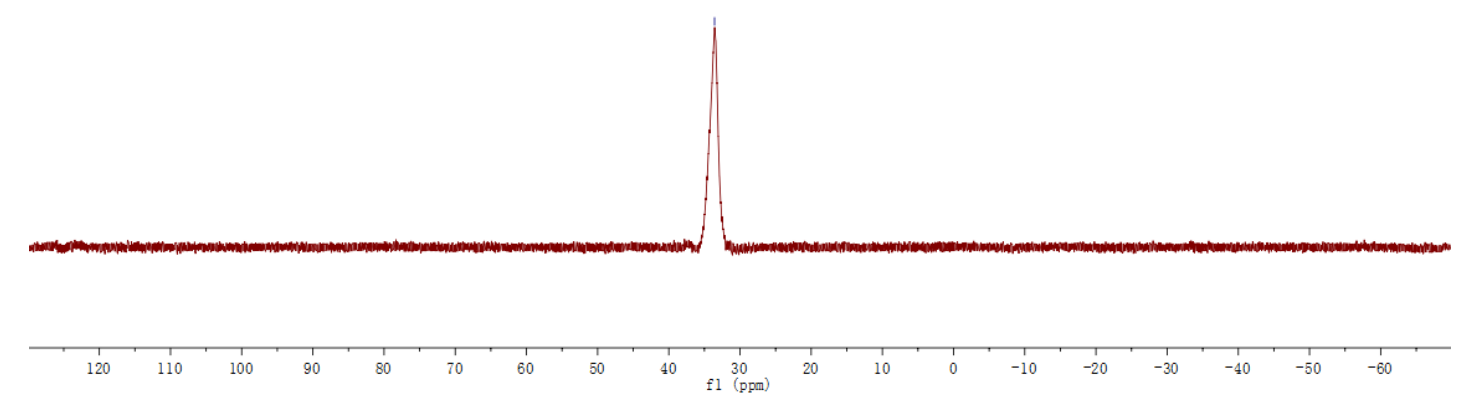

${ }^{19} \mathrm{~F}$ NMR Spectrum of Compound $4 \mathrm{e}\left(376 \mathrm{MHz}, \mathrm{CDCl}_{3}\right)$<smiles>CC1(C)OB(C[C@]2(OC(F)(F)F)C(=O)N(Cc3ccccc3)c3ccc(OC(F)(F)F)cc32)OC1(C)C</smiles>

$4 e$

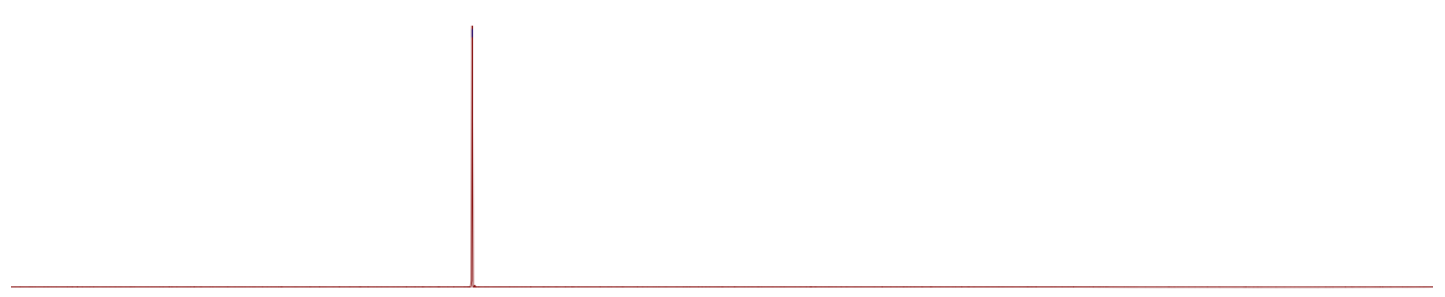




\section{'H NMR Spectrum of Compound 4f (400 MHz, DMSO-d6)}

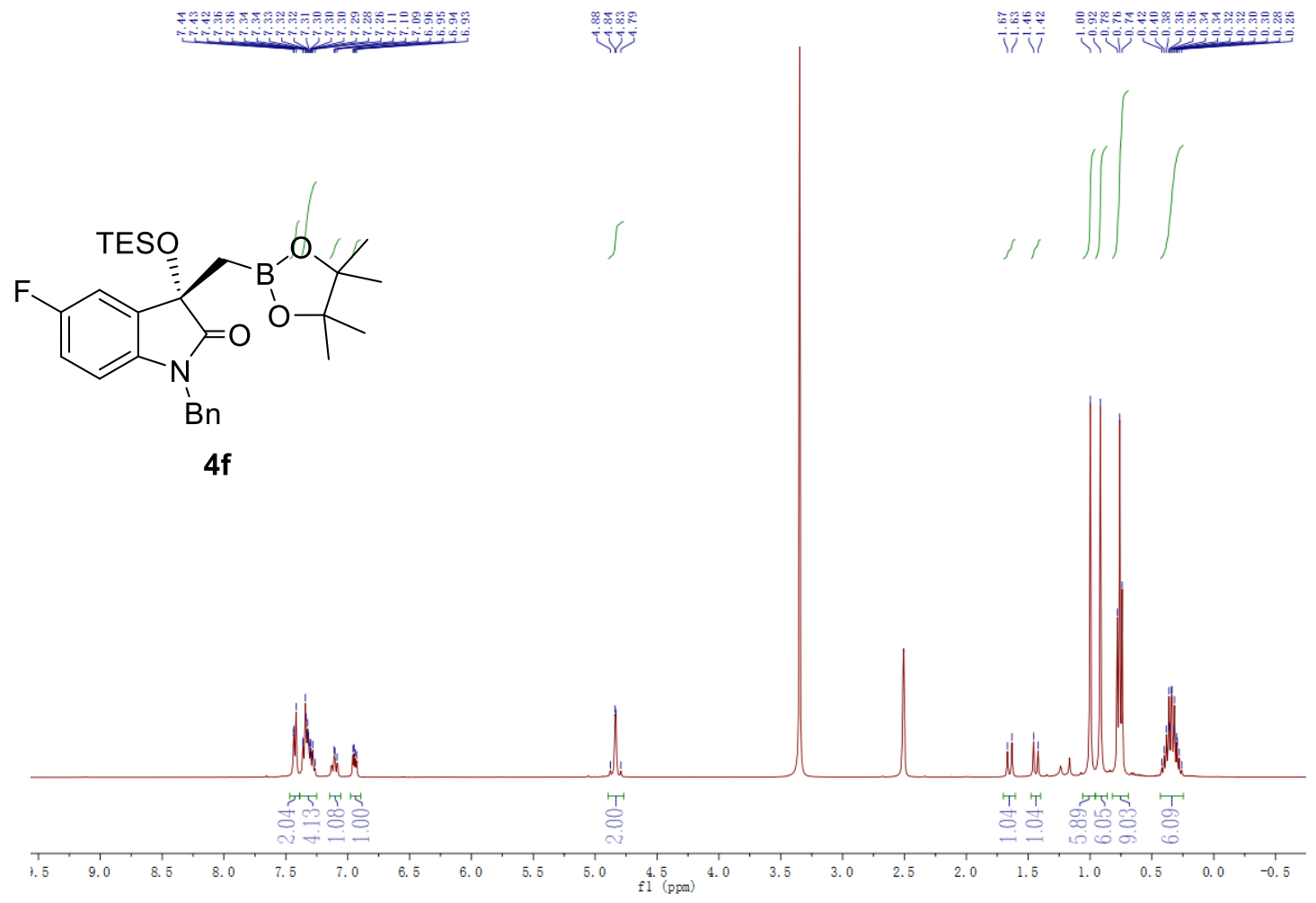

${ }^{13} \mathrm{C}$ NMR Spectrum of Compound $4 \mathrm{f}$ (101 MHz, DMSO-d6)

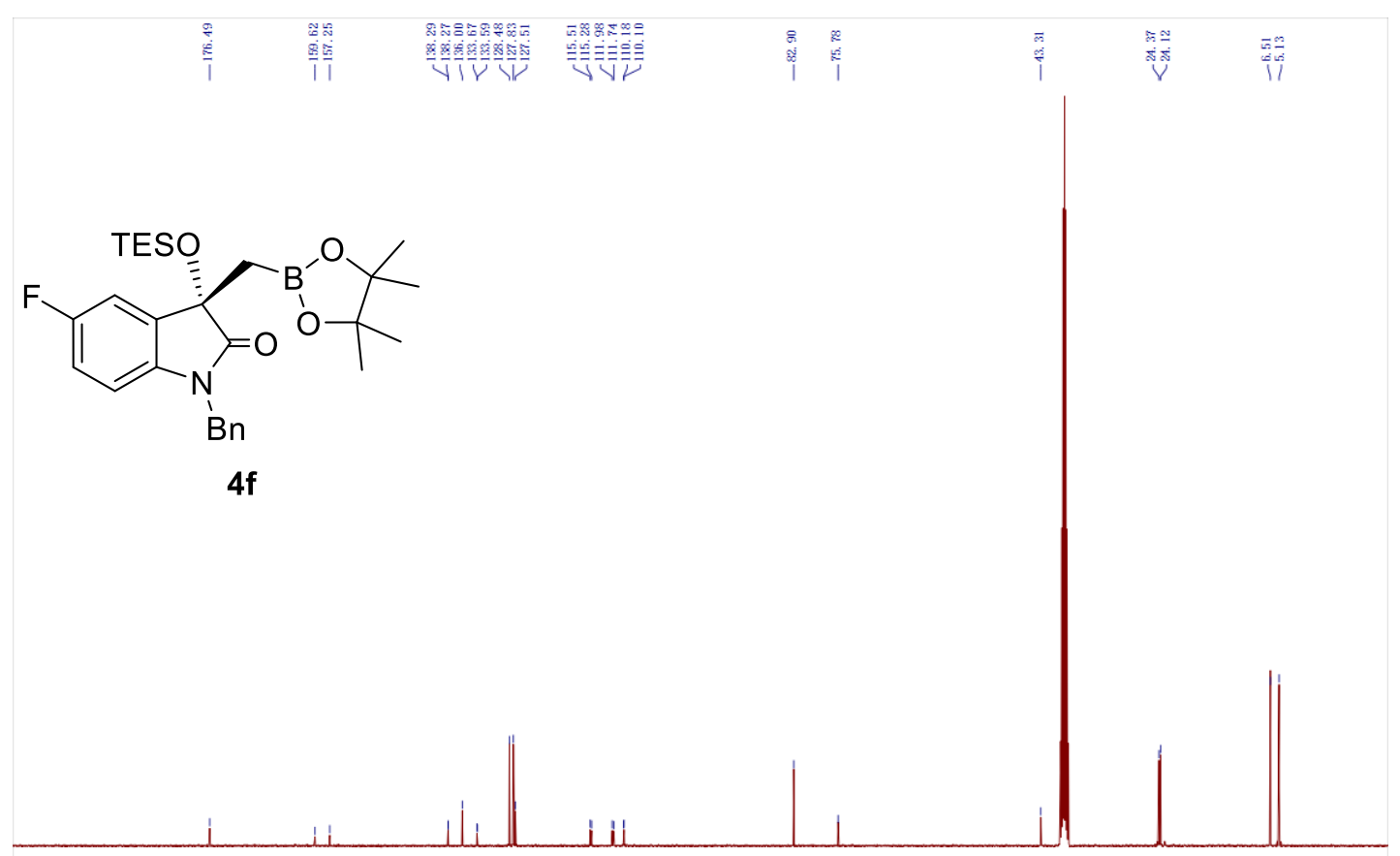

$\begin{array}{lllllllllll}200 & 190 & 180 & 170 & 160 & 150 & 140 & 130 & 120 & 110 & 100 \\ \mathrm{f} 1(\mathrm{ppm}) & 90\end{array}$ 
11B NMR Spectrum of Compound $4 \mathrm{f}$ (193 MHz, DMSO-d6)
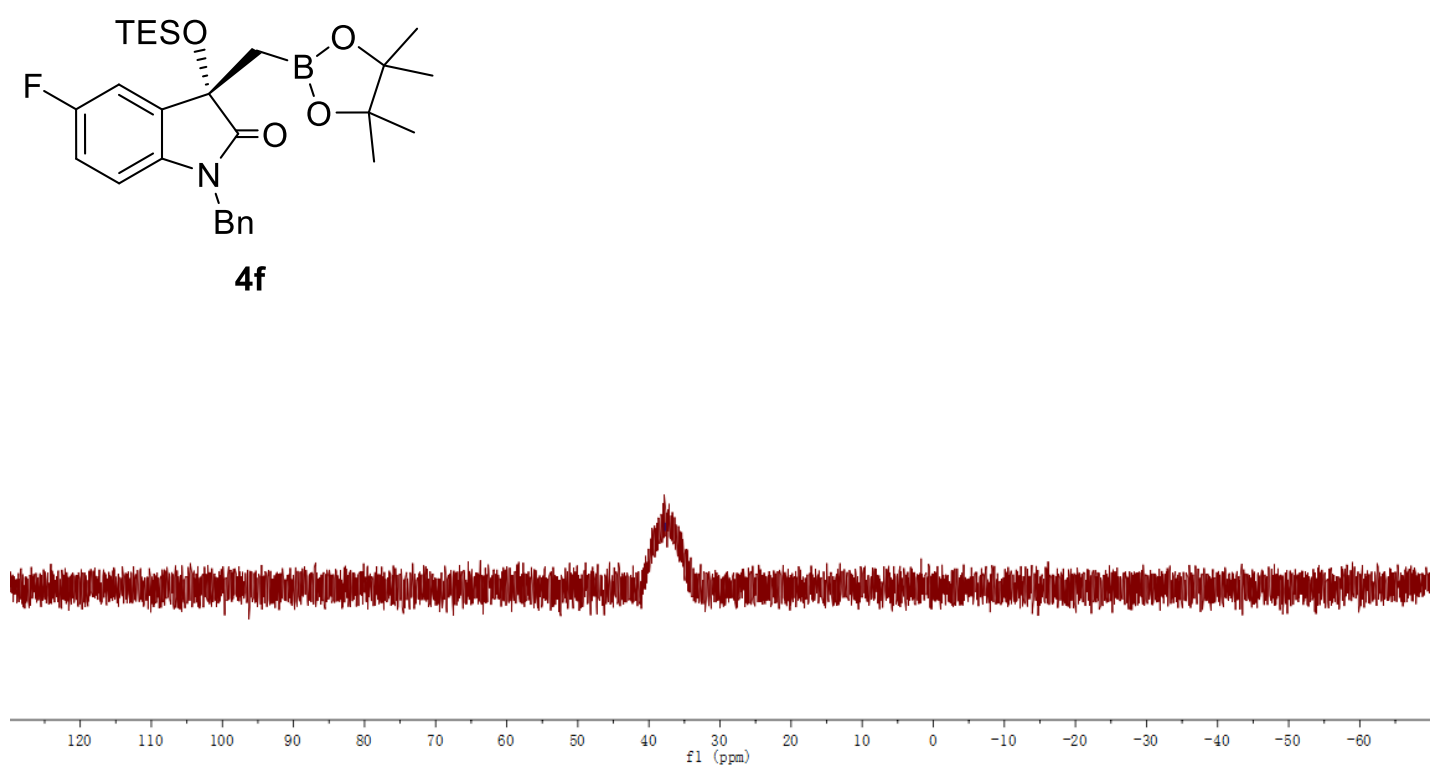

${ }^{19}$ F NMR Spectrum of Compound $4 f$ (376 MHz, DMSO-d6)

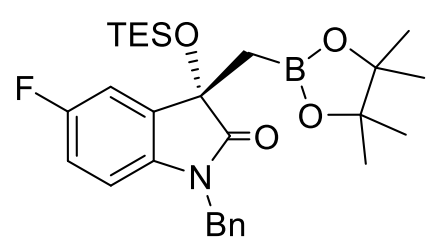

$4 f$

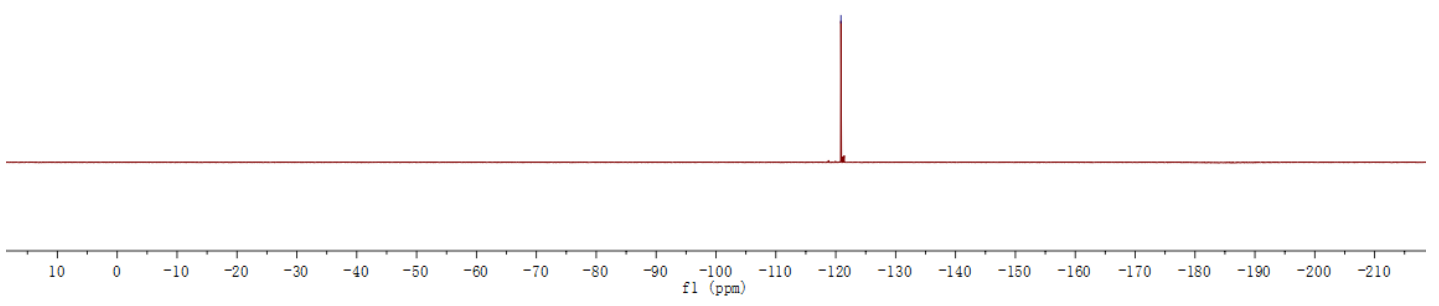


${ }^{1} \mathrm{H}$ NMR Spectrum of Compound $4 \mathrm{~g}$ (400 MHz, DMSO-d6)

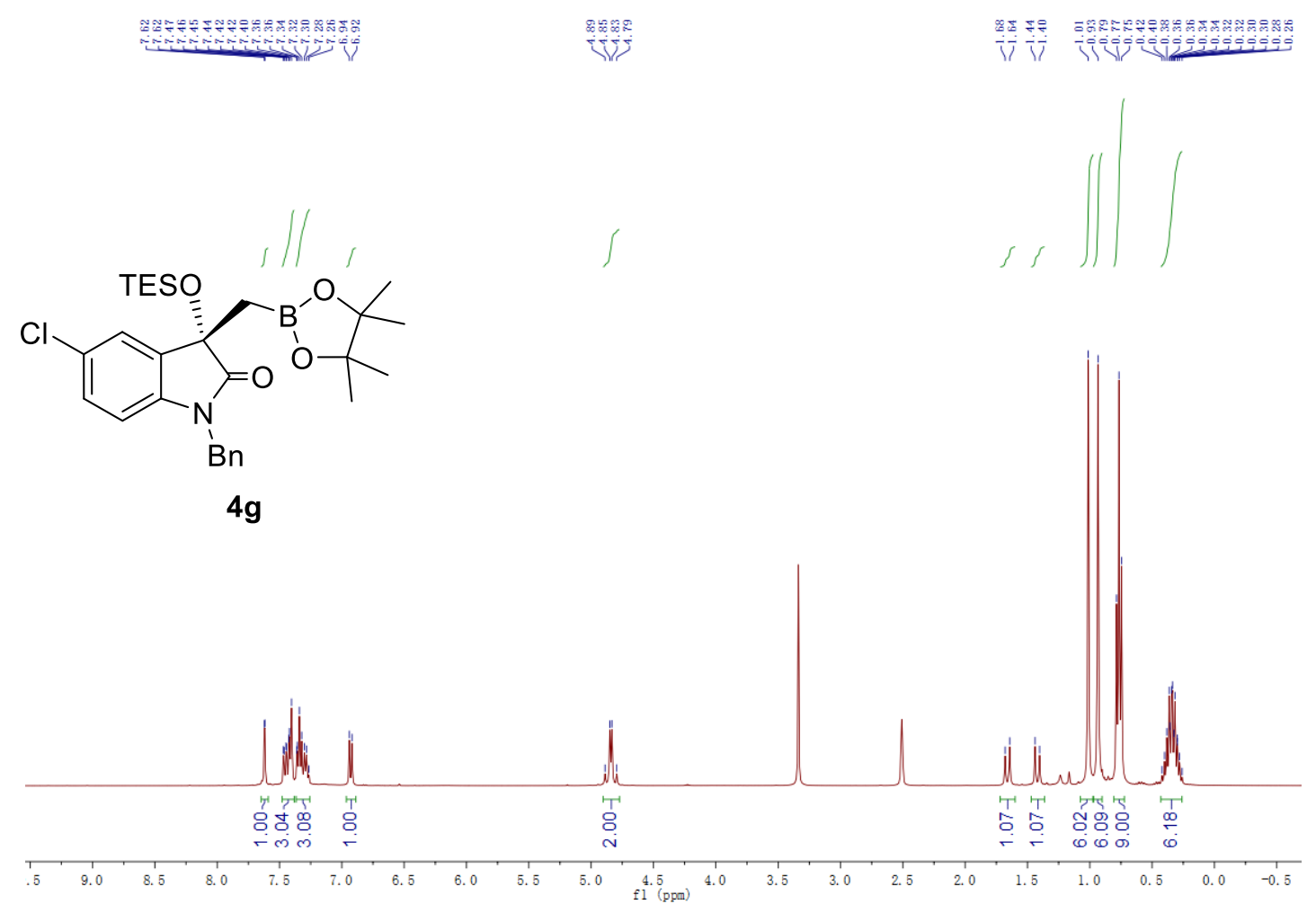

${ }^{13} \mathrm{C}$ NMR Spectrum of Compound $4 \mathrm{~g}$ (101 MHz, DMSO-d6)
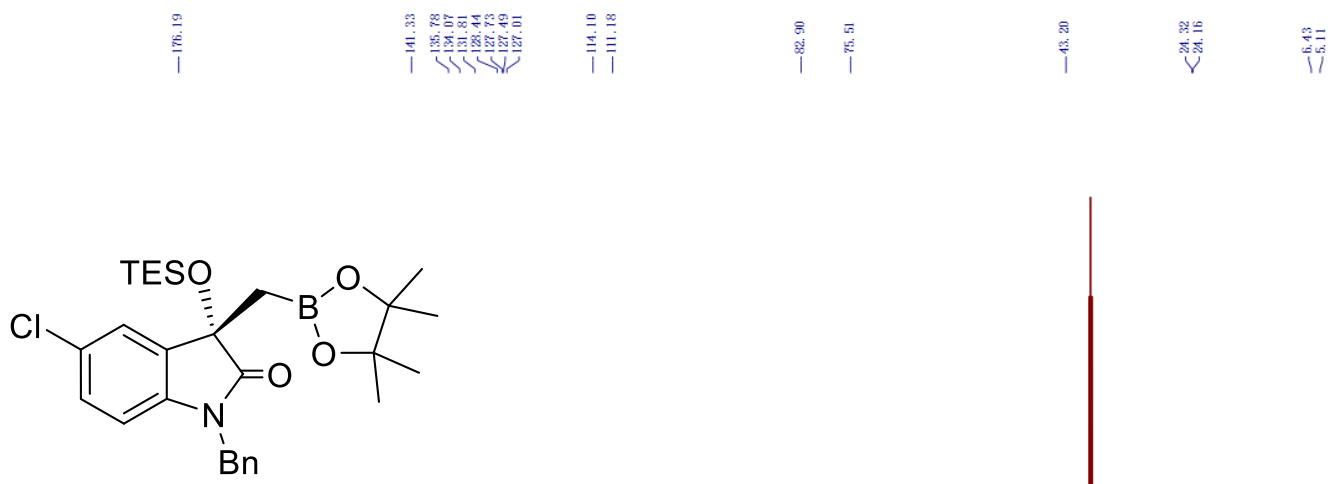

$4 g$
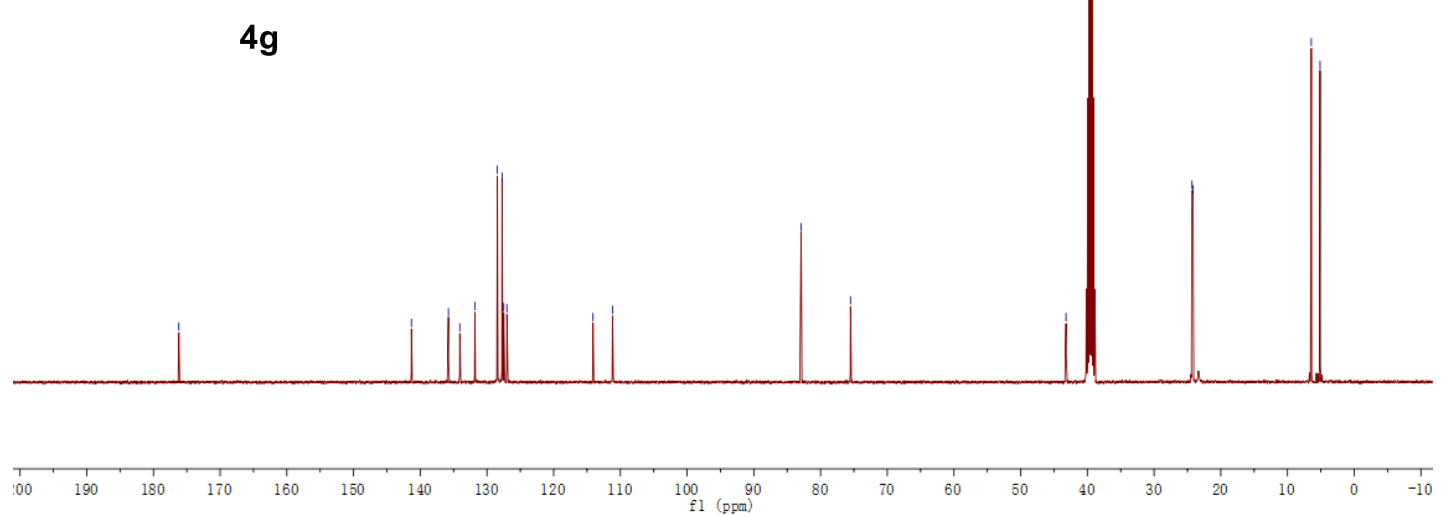
11B NMR Spectrum of Compound 4g (193 MHz, DMSO-d6)

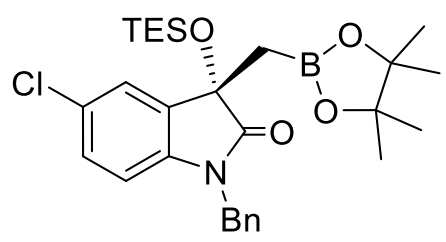

$4 g$

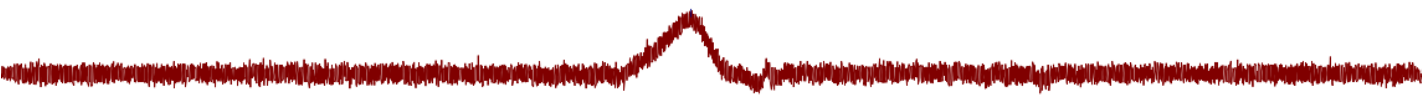


${ }^{1} \mathrm{H}$ NMR Spectrum of Compound $4 \mathrm{~h}(400 \mathrm{MHz}$, DMSO-d6)

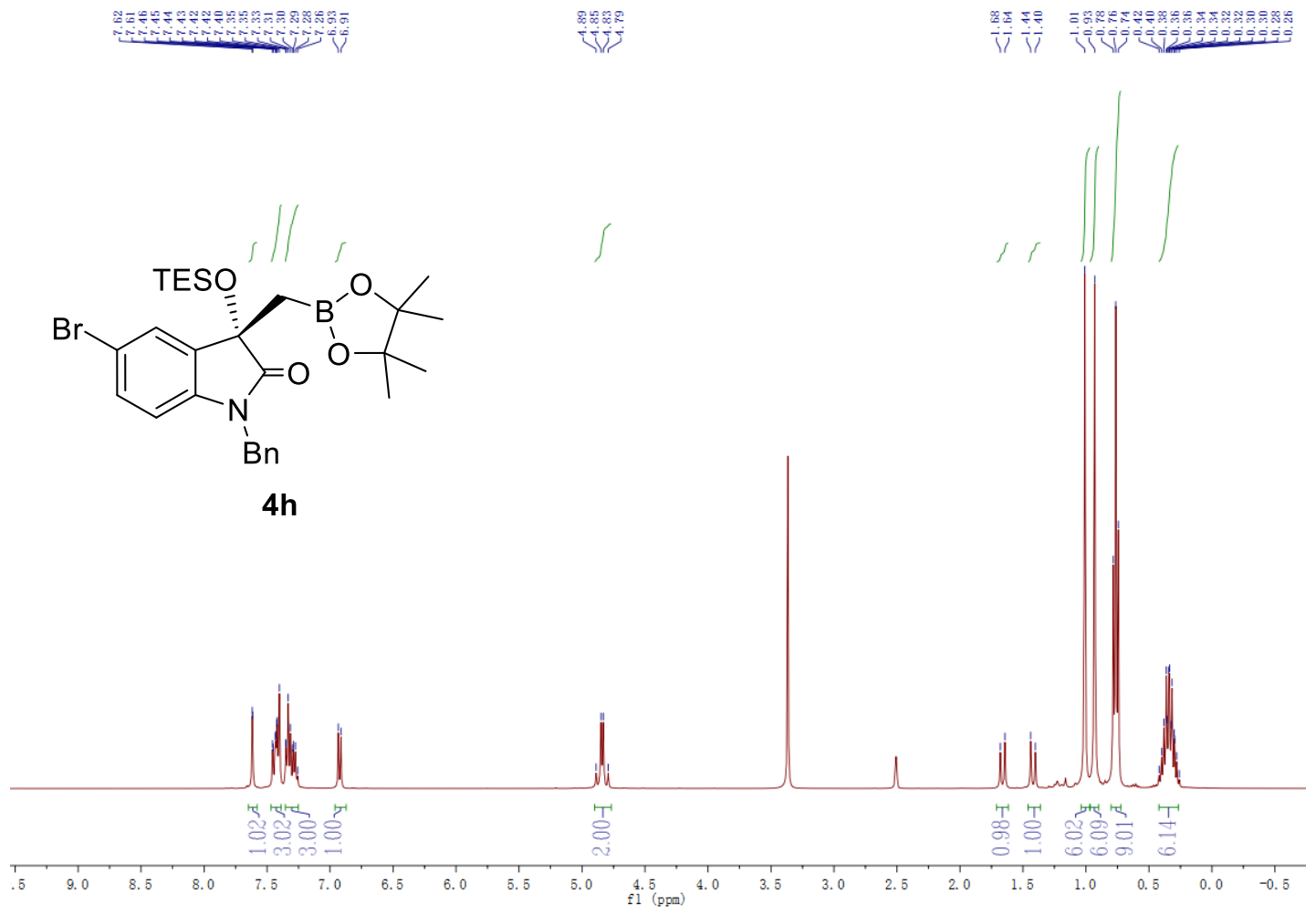

${ }^{13} \mathrm{C}$ NMR Spectrum of Compound 4h (101 MHz, DMSO-d6) i

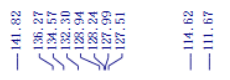<smiles>CC1(C)OB(C[C@]2(OS(F)(F)F)C(=O)N(Cc3ccccc3)c3ccc(Br)cc32)OC1(C)C</smiles>

$4 h$
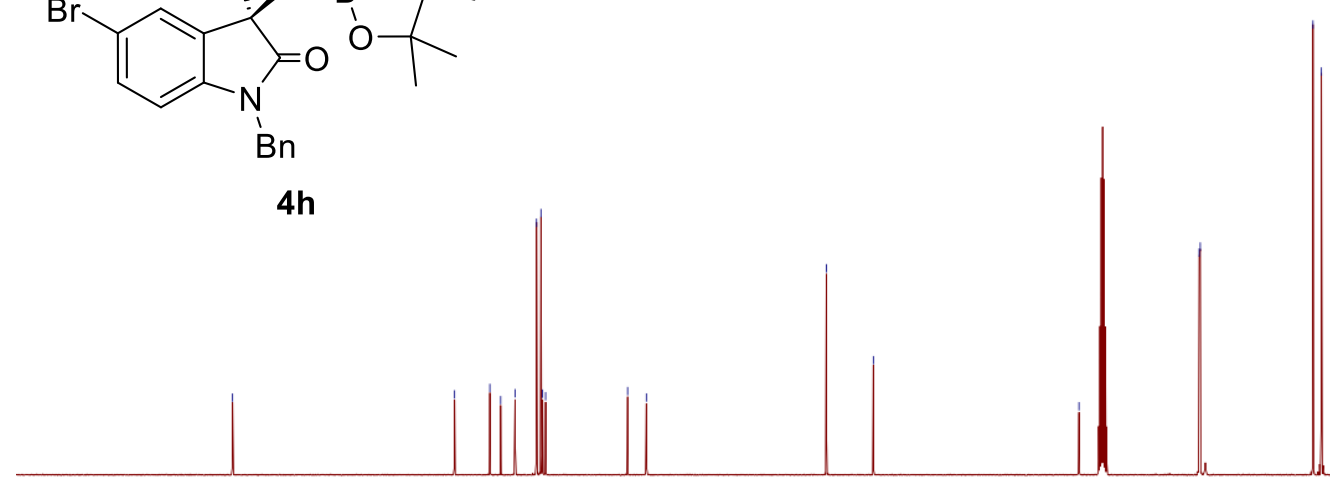
${ }^{11} \mathrm{~B}$ NMR Spectrum of Compound 4h (193 MHz, DMSO-d6)

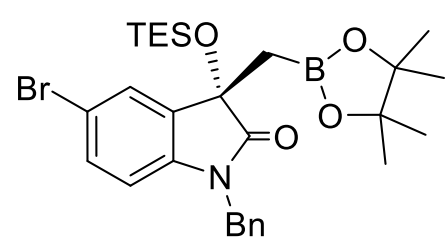

$4 h$

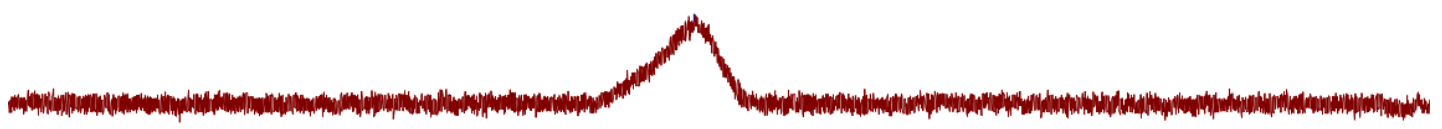


${ }^{1} \mathrm{H}$ NMR Spectrum of Compound 4i (400 MHz, DMSO-d6)

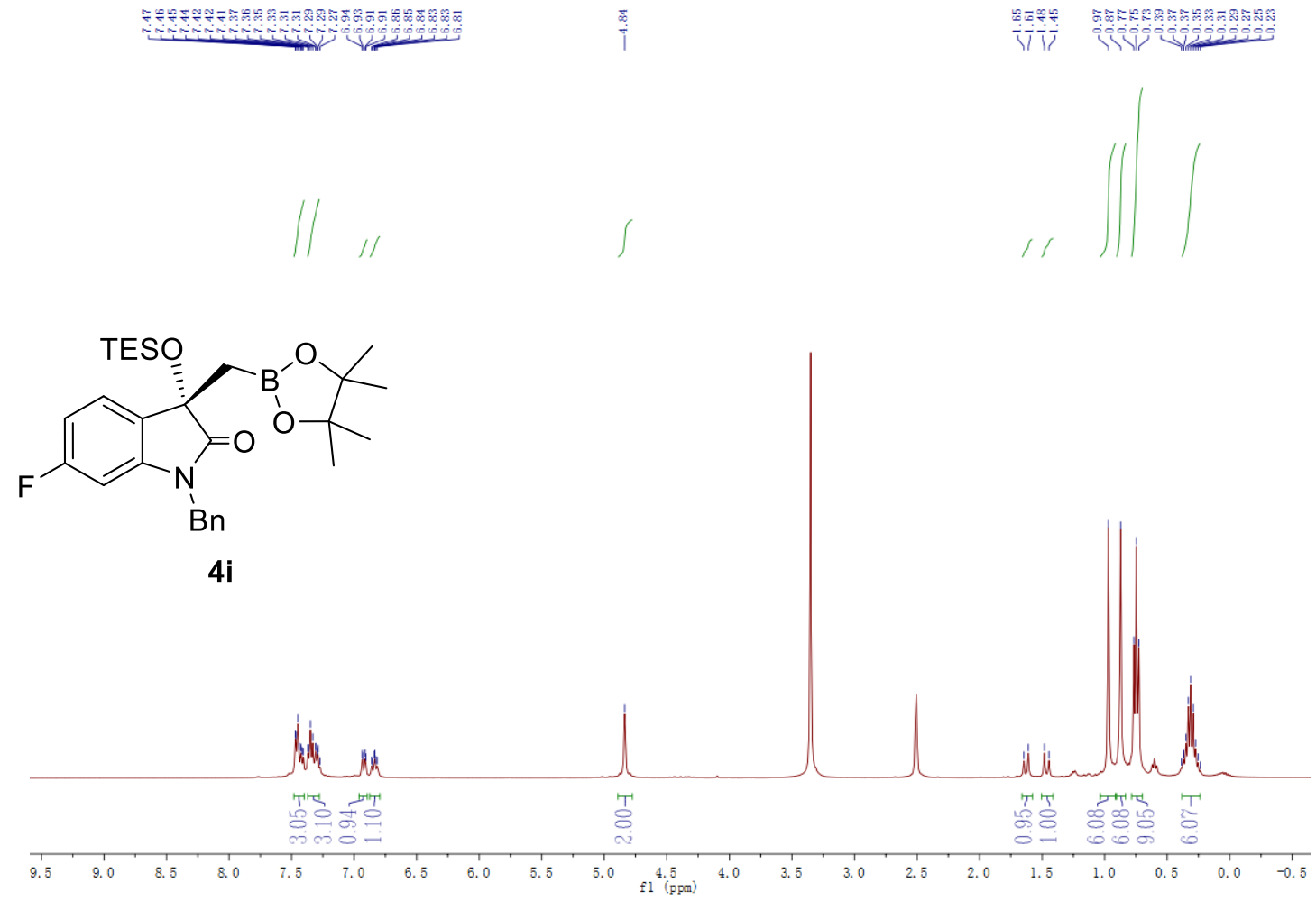

${ }^{13} \mathrm{C}$ NMR Spectrum of Compound $4 \mathrm{i}$ (101 MHz, DMSO-d6)

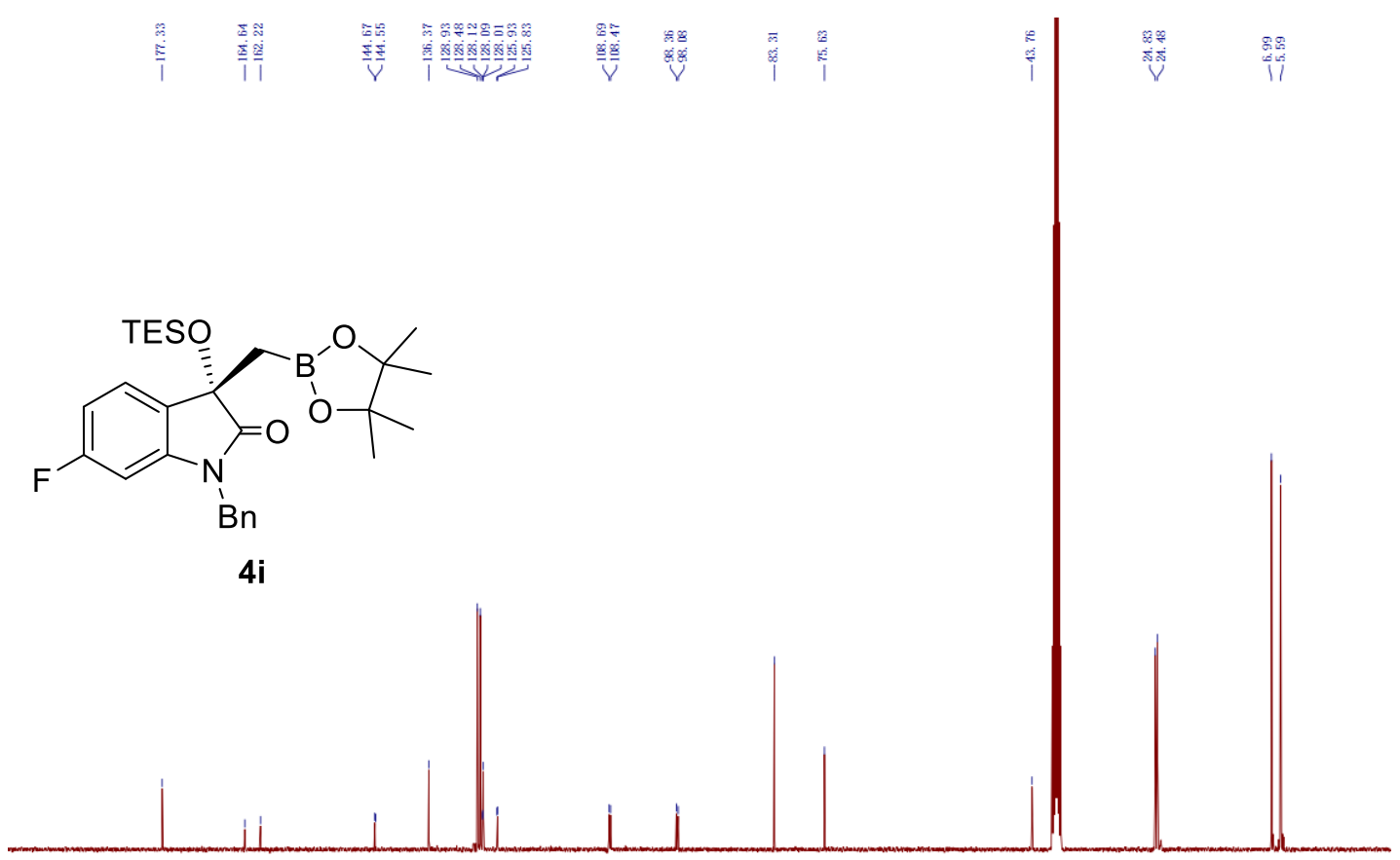

$\begin{array}{llllllllll}190 & 180 & 170 & 160 & 150 & 140 & 130 & 120 & 110 & 100 \\ \mathrm{f} 1(\mathrm{ppm}) & 90\end{array}$ 
11B NMR Spectrum of Compound 4i (193 MHz, DMSO-d6)

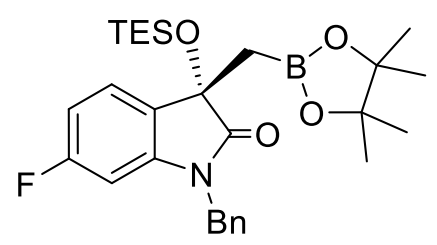

$4 \mathbf{i}$

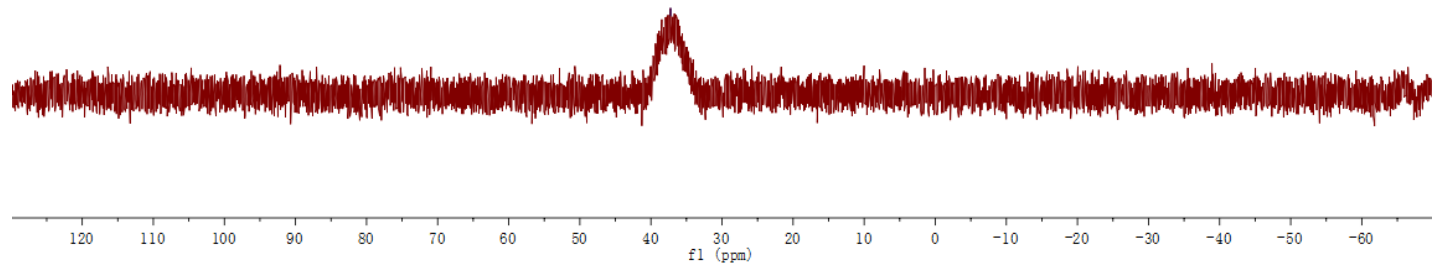

${ }^{19} \mathrm{~F}$ NMR Spectrum of Compound 4i $\left(376 \mathrm{MHz}, \mathrm{CDCl}_{3}\right)$<smiles>CCO[C@]1(CB=O)C(=O)Nc2cc(F)ccc21</smiles>

$4 \mathbf{i}$

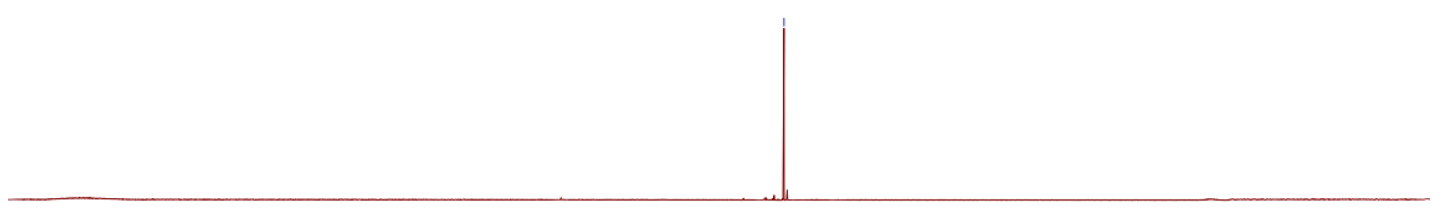

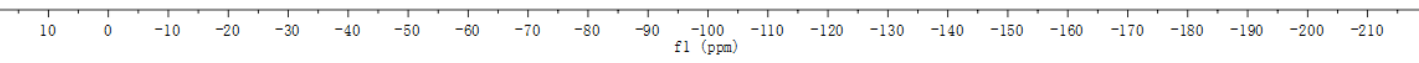


${ }^{1} \mathrm{H}$ NMR Spectrum of Compound 4j (400 MHz, DMSO-d6)

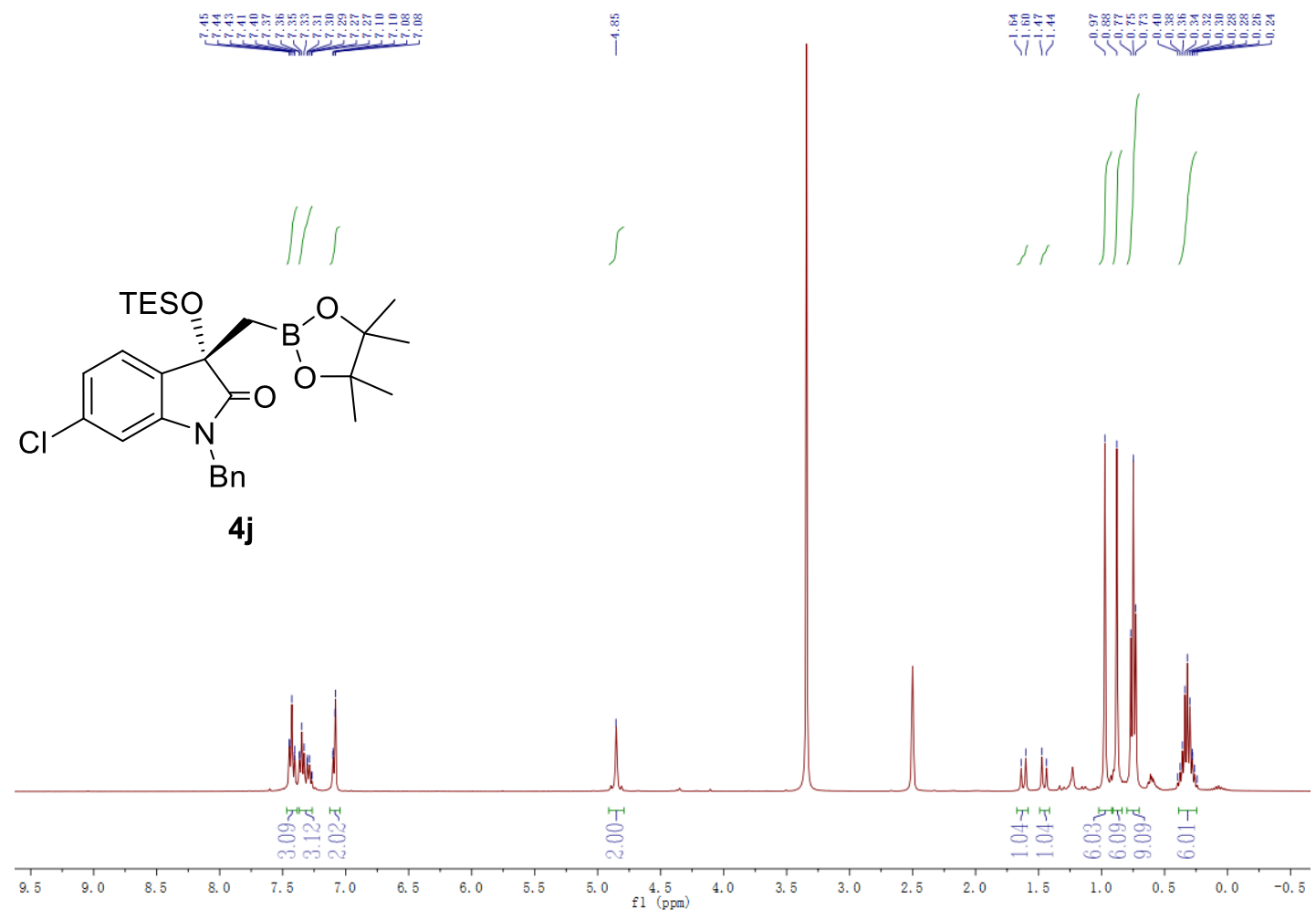

${ }^{13} \mathrm{C}$ NMR Spectrum of Compound $4 \mathrm{j}$ (101 MHz, DMSO-d6)

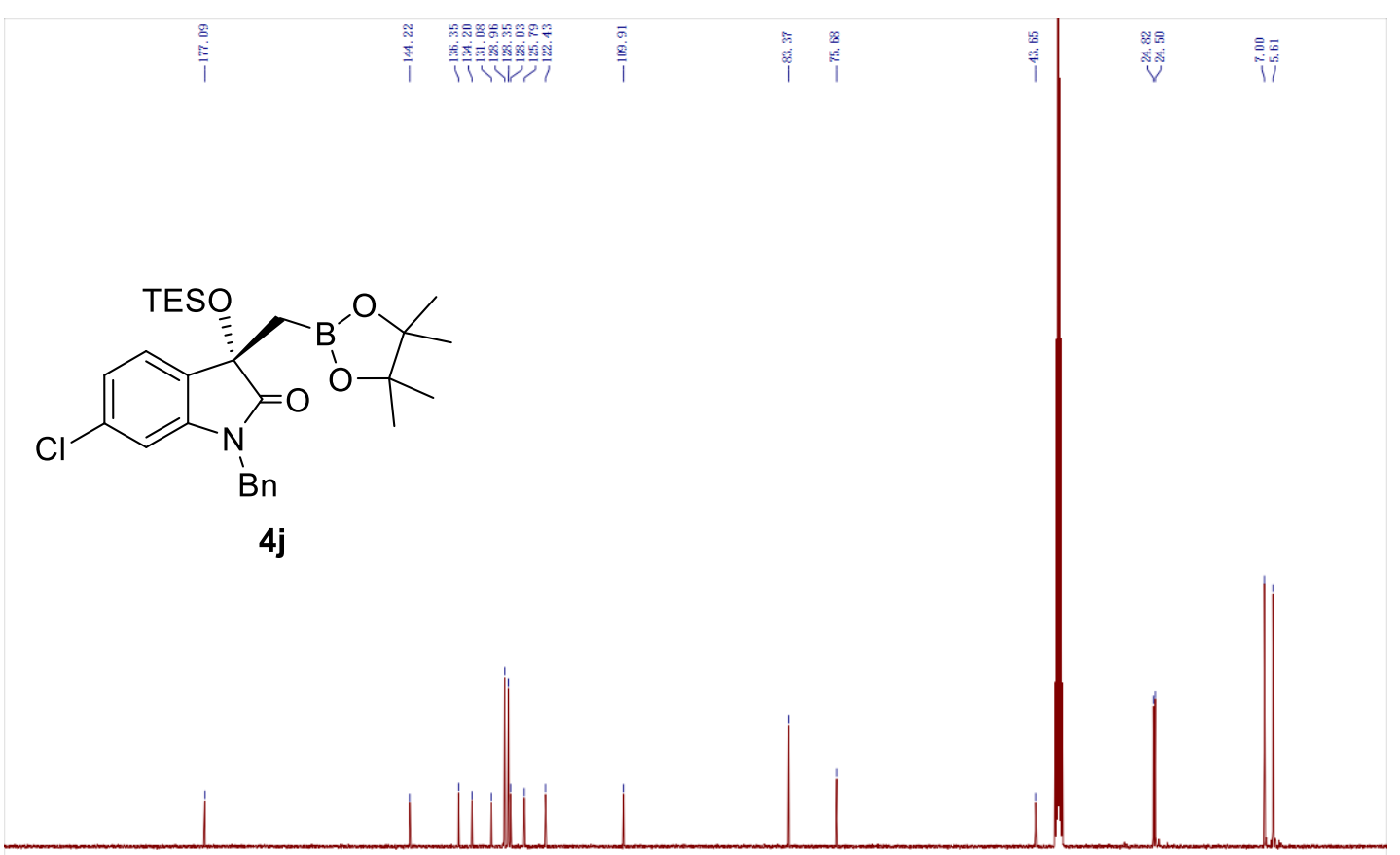

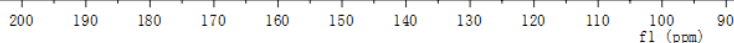


${ }^{11} \mathrm{~B}$ NMR Spectrum of Compound 4j (193 MHz, $\left.\mathrm{CDCl}_{3}\right)$

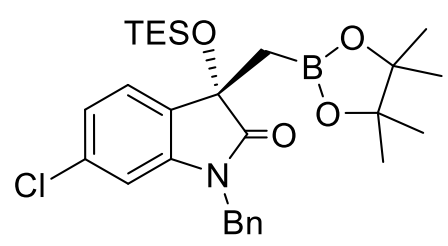

$4 \mathrm{j}$

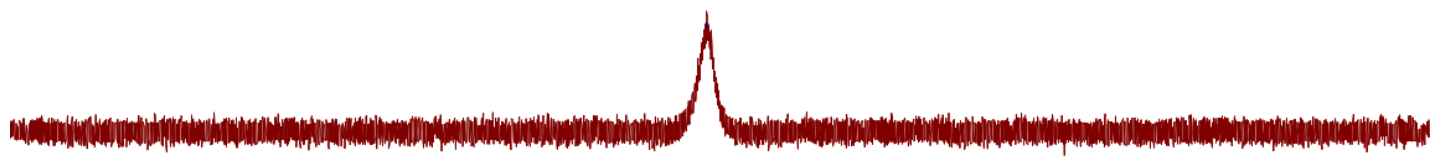


${ }^{1} \mathrm{H}$ NMR Spectrum of Compound 4k (400 MHz, DMSO-d6)

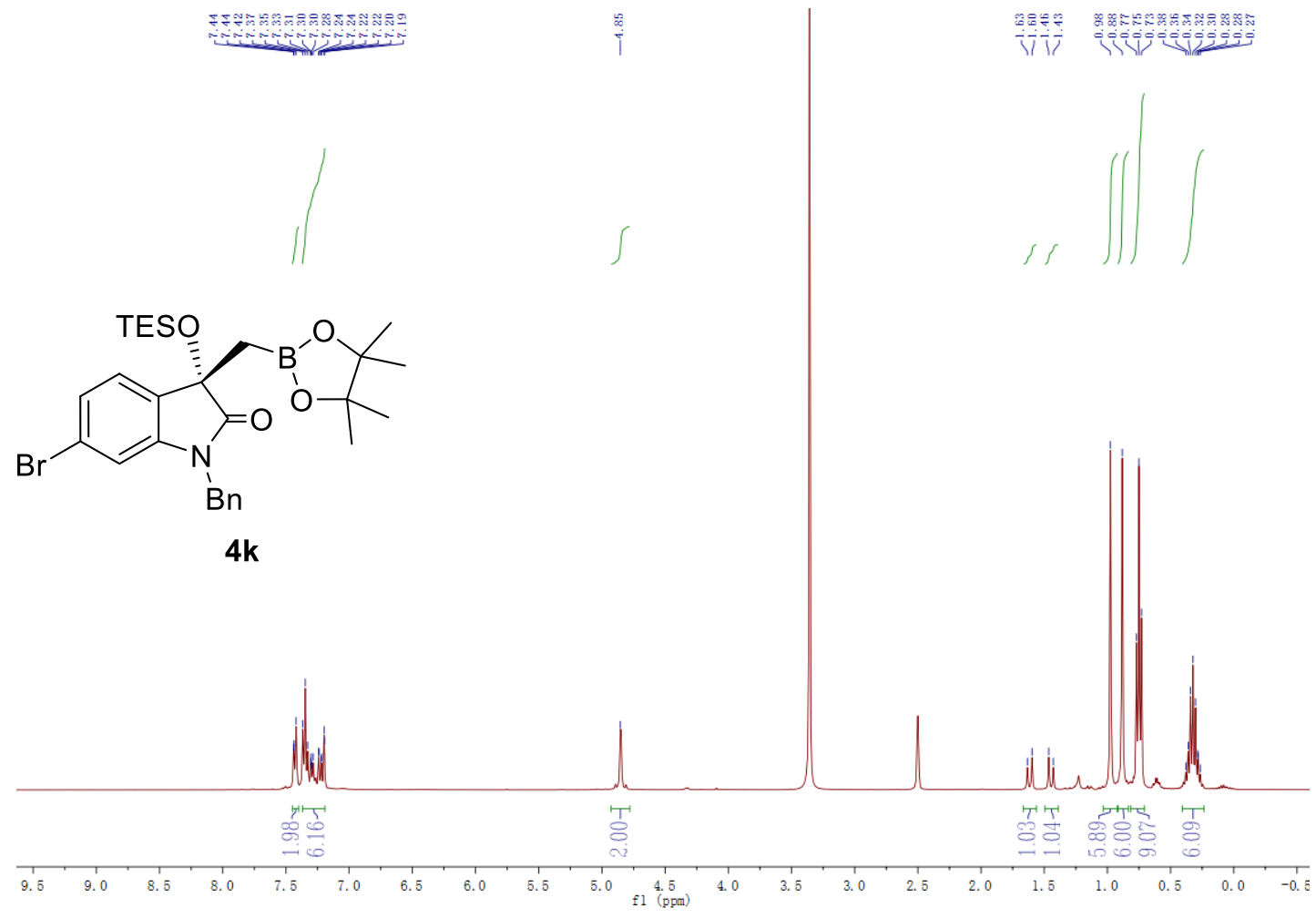

${ }^{13} \mathrm{C}$ NMR Spectrum of Compound 4k (101 MHz, DMSO-d6)

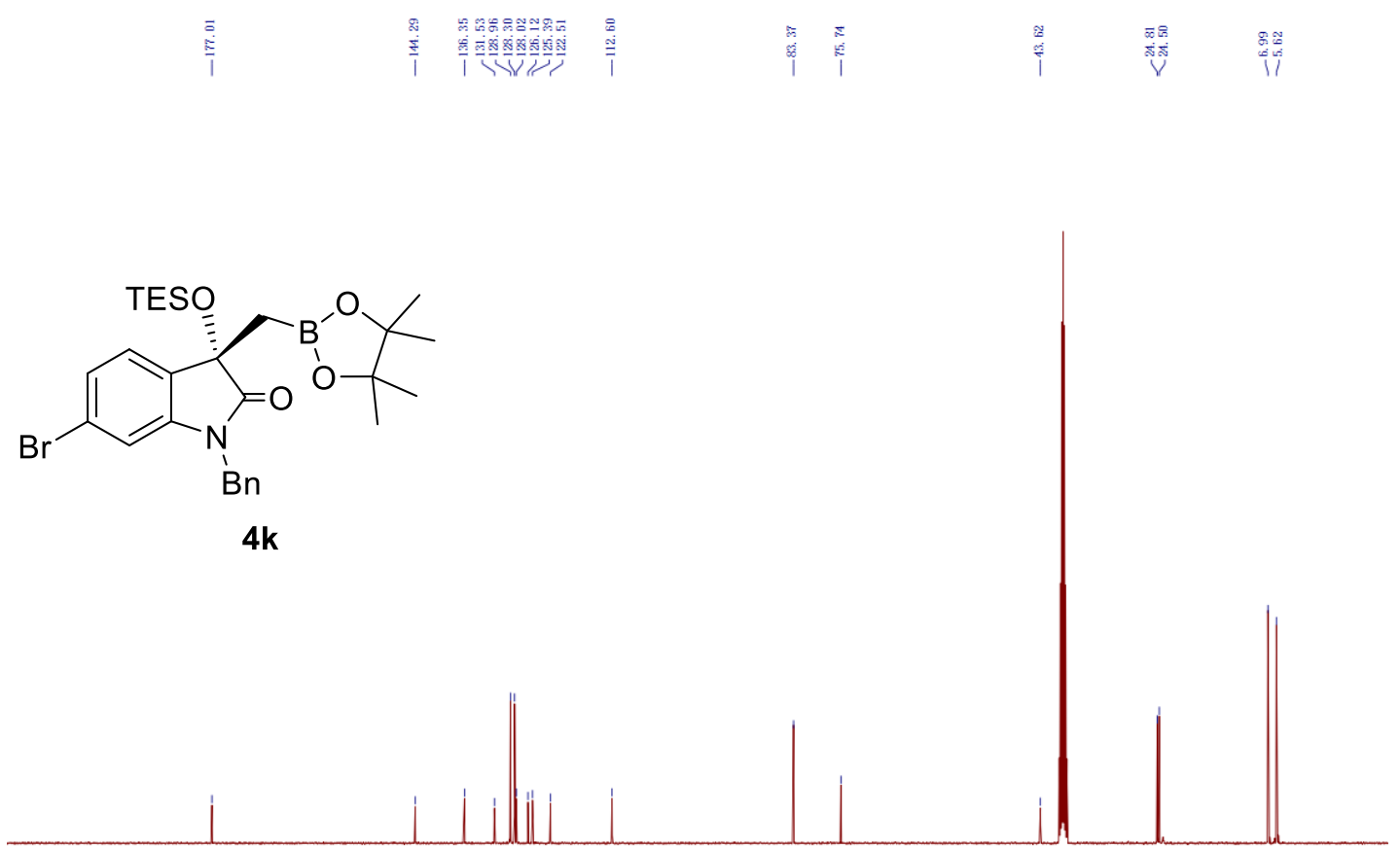

$\begin{array}{lllllllllllll}1 & 200 & 190 & 180 & 170 & 160 & 150 & 140 & 130 & 120 & 110 & 100 & 1 \\ \mathrm{f} 1(\mathrm{ppm}) & 90\end{array}$ 
11B NMR Spectrum of Compound 4k (193 MHz, DMSO-d6)

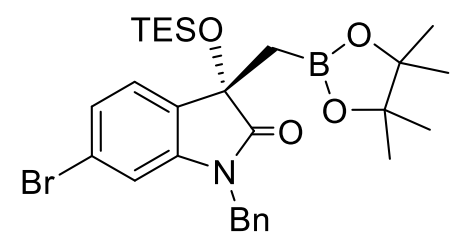

$4 k$

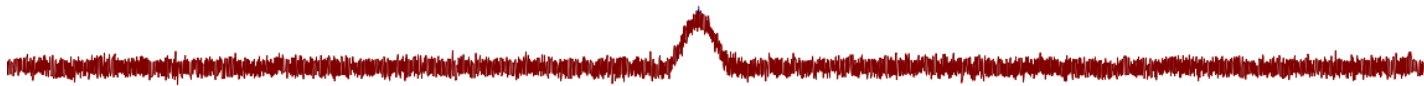


${ }^{1} \mathrm{H}$ NMR Spectrum of Compound $4 \mathrm{l}$ (400 MHz, DMSO-d6)

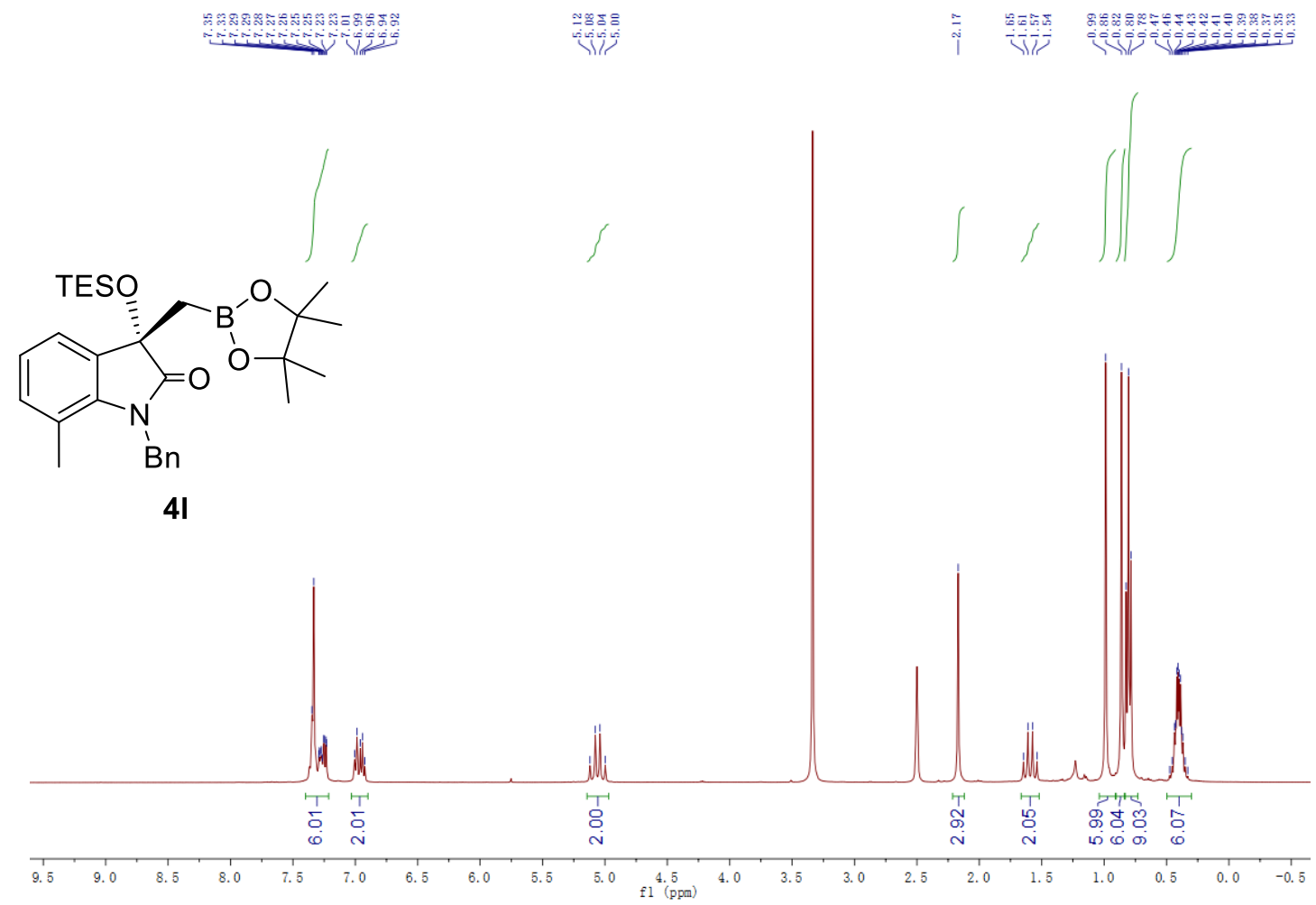

${ }^{13} \mathrm{C}$ NMR Spectrum of Compound 4I (101 MHz, DMSO-d6)
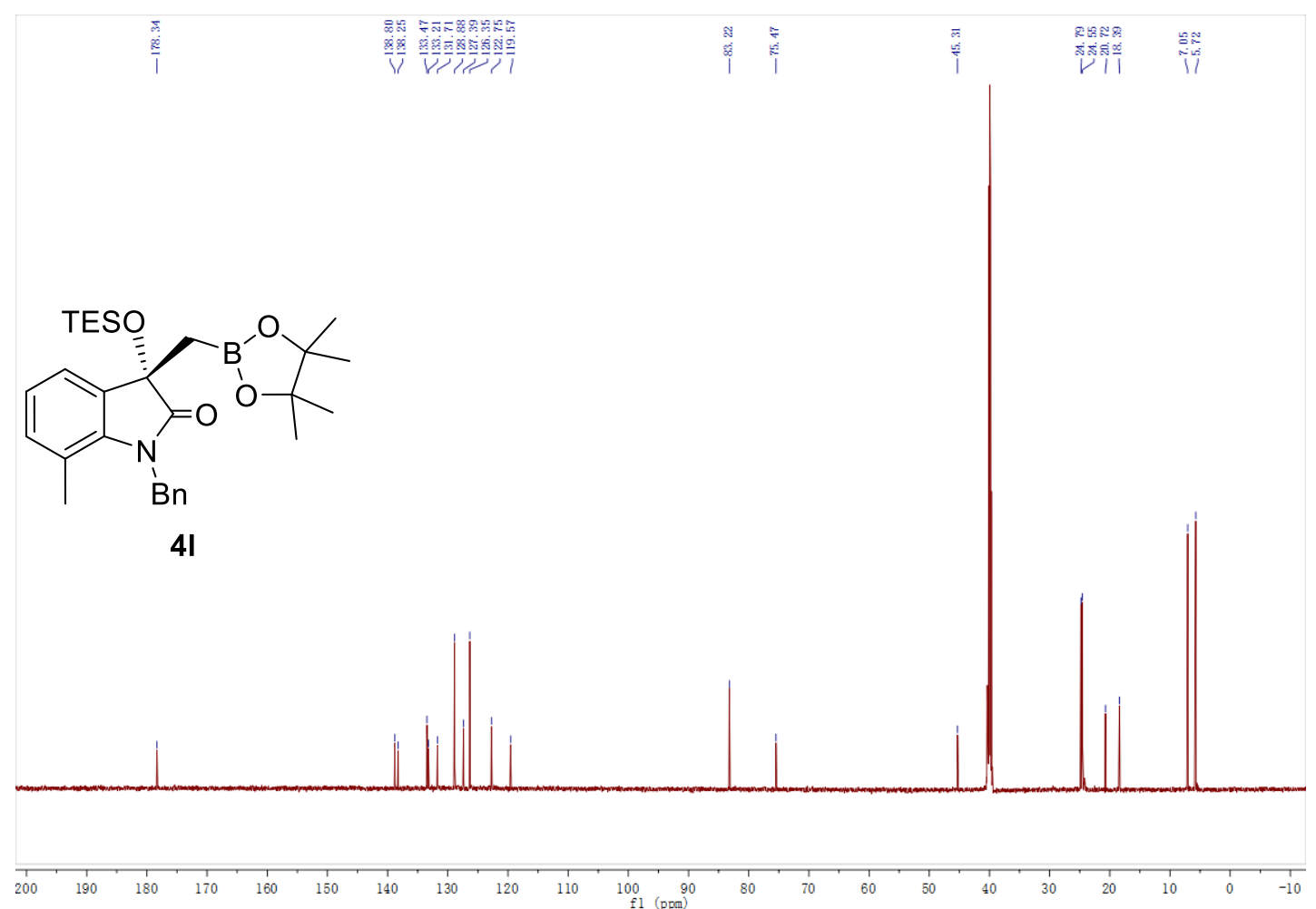
${ }^{11}$ B NMR Spectrum of Compound 4I (193 MHz, DMSO-d6)

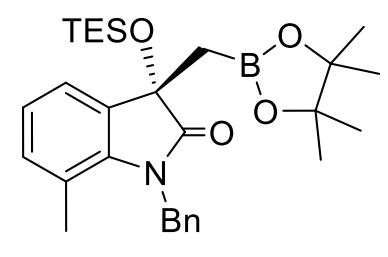

4

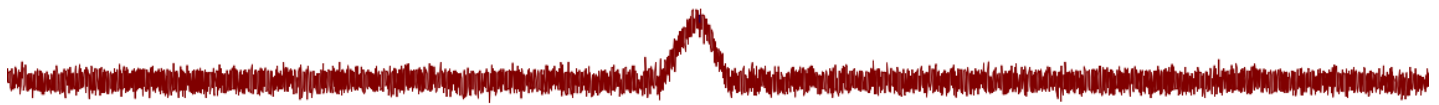


${ }^{1} \mathrm{H}$ NMR Spectrum of Compound $4 \mathrm{~m}$ (400 MHz, DMSO-d6)

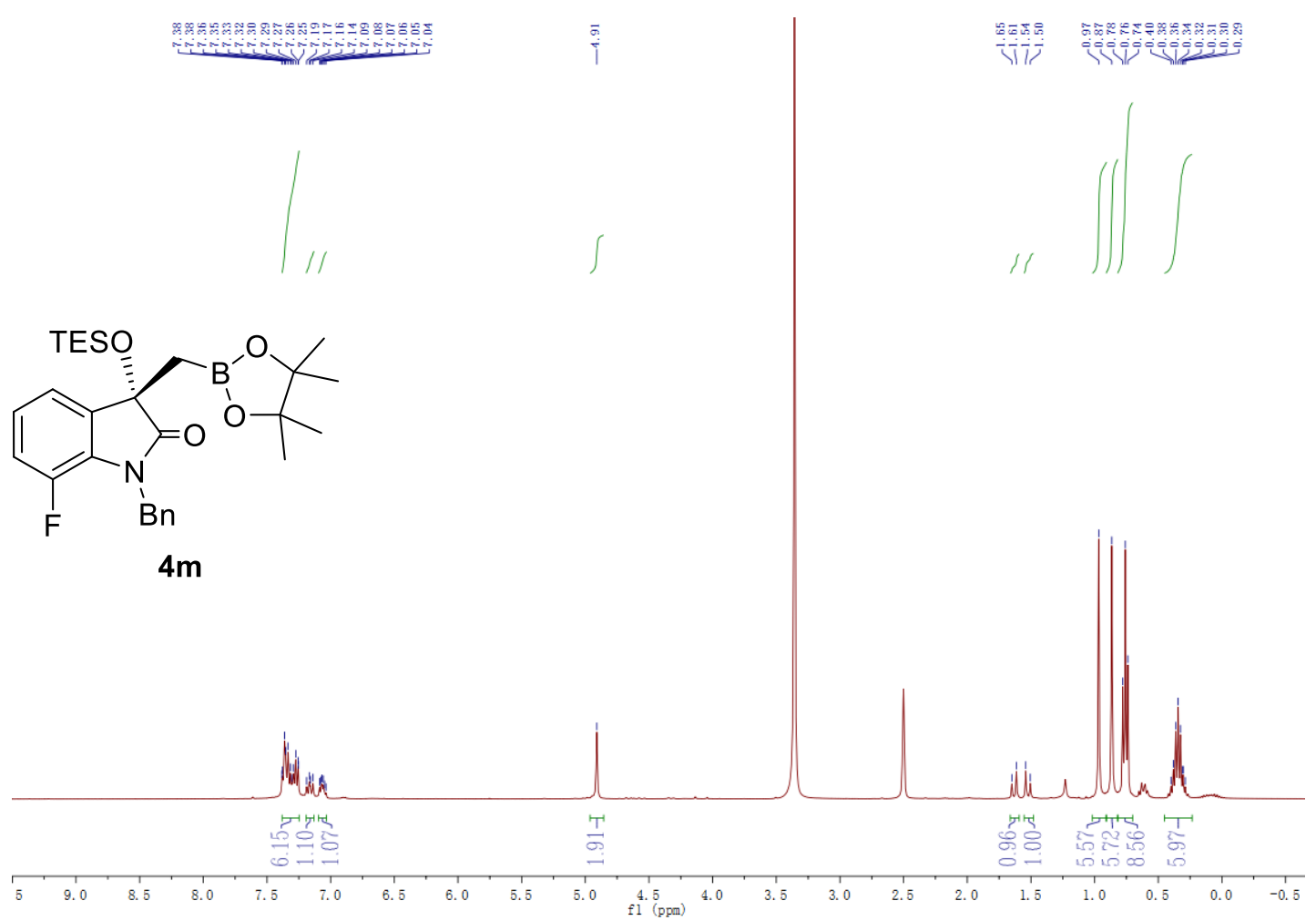

${ }^{13} \mathrm{C}$ NMR Spectrum of Compound $4 \mathrm{~m}$ (101 MHz, DMSO-d6)

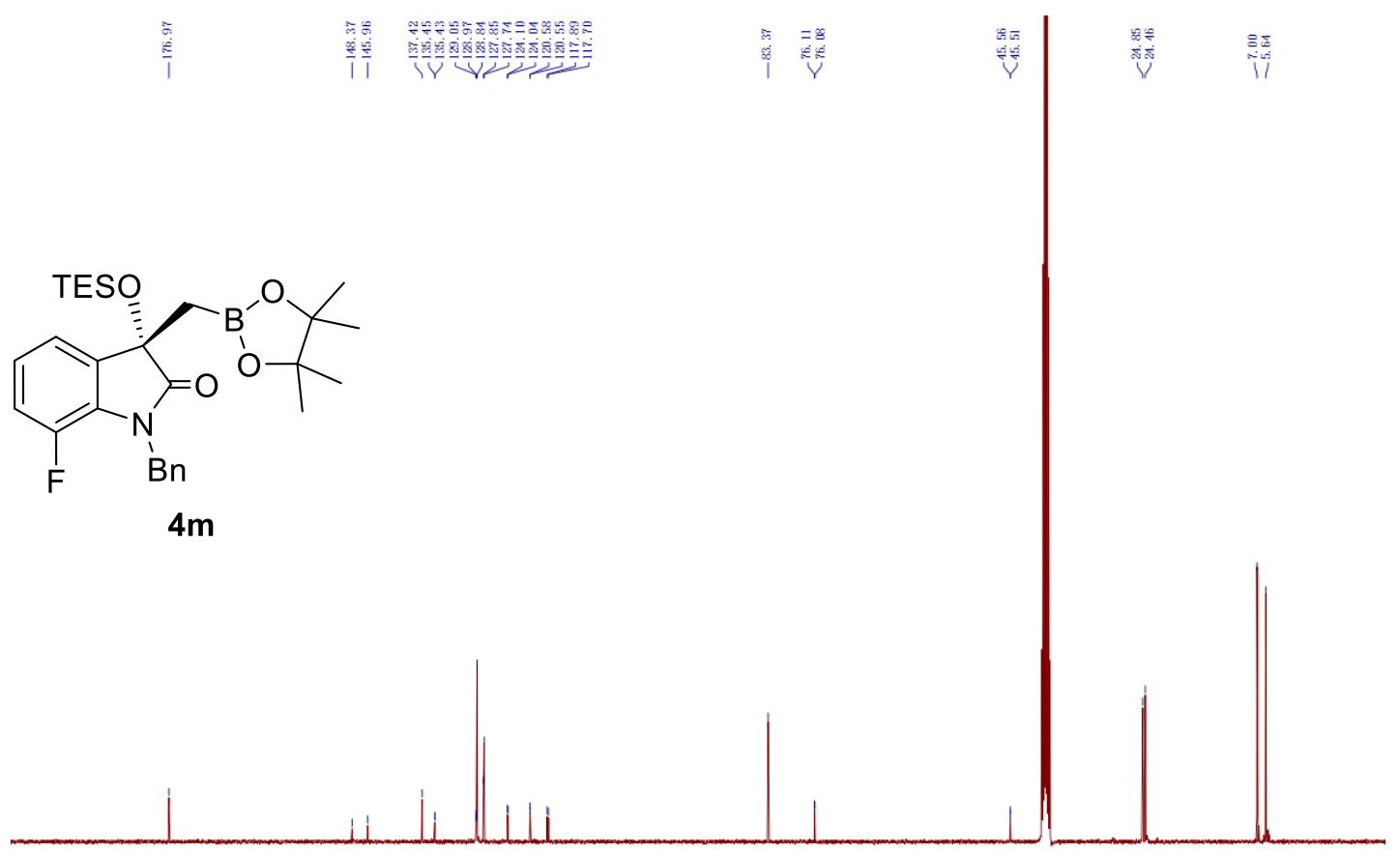

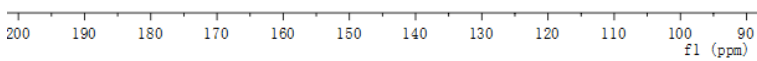


${ }^{11} \mathrm{~B}$ NMR Spectrum of Compound $4 \mathrm{~m}\left(193 \mathrm{MHz}, \mathrm{CDCl}_{3}\right)$<smiles>CCO[C@@]1(CB2OC(C)(C)C(C)(C)O2)C(=O)N(Cc2ccccc2)c2c(F)cccc21</smiles>
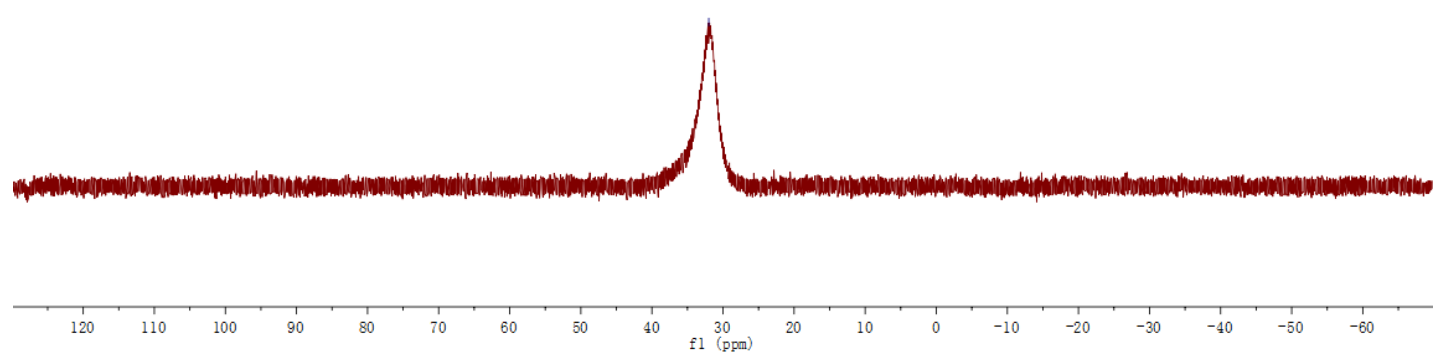

${ }^{19} \mathrm{~F}$ NMR Spectrum of Compound $4 \mathrm{e}\left(376 \mathrm{MHz} \mathrm{CDCl}_{3}\right)$<smiles>CCO[C@]1(CB2OC(C)(C)C(C)(C)O2)C(=O)N(Cc2ccccc2)c2c(F)cccc21</smiles>

$4 m$

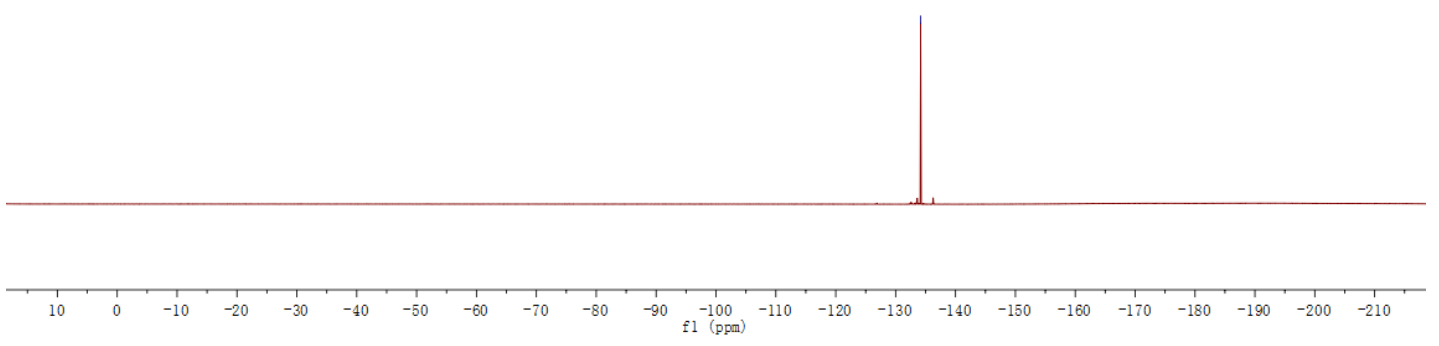


${ }^{1} \mathrm{H}$ NMR Spectrum of Compound 4n (400 MHz, DMSO-d6)

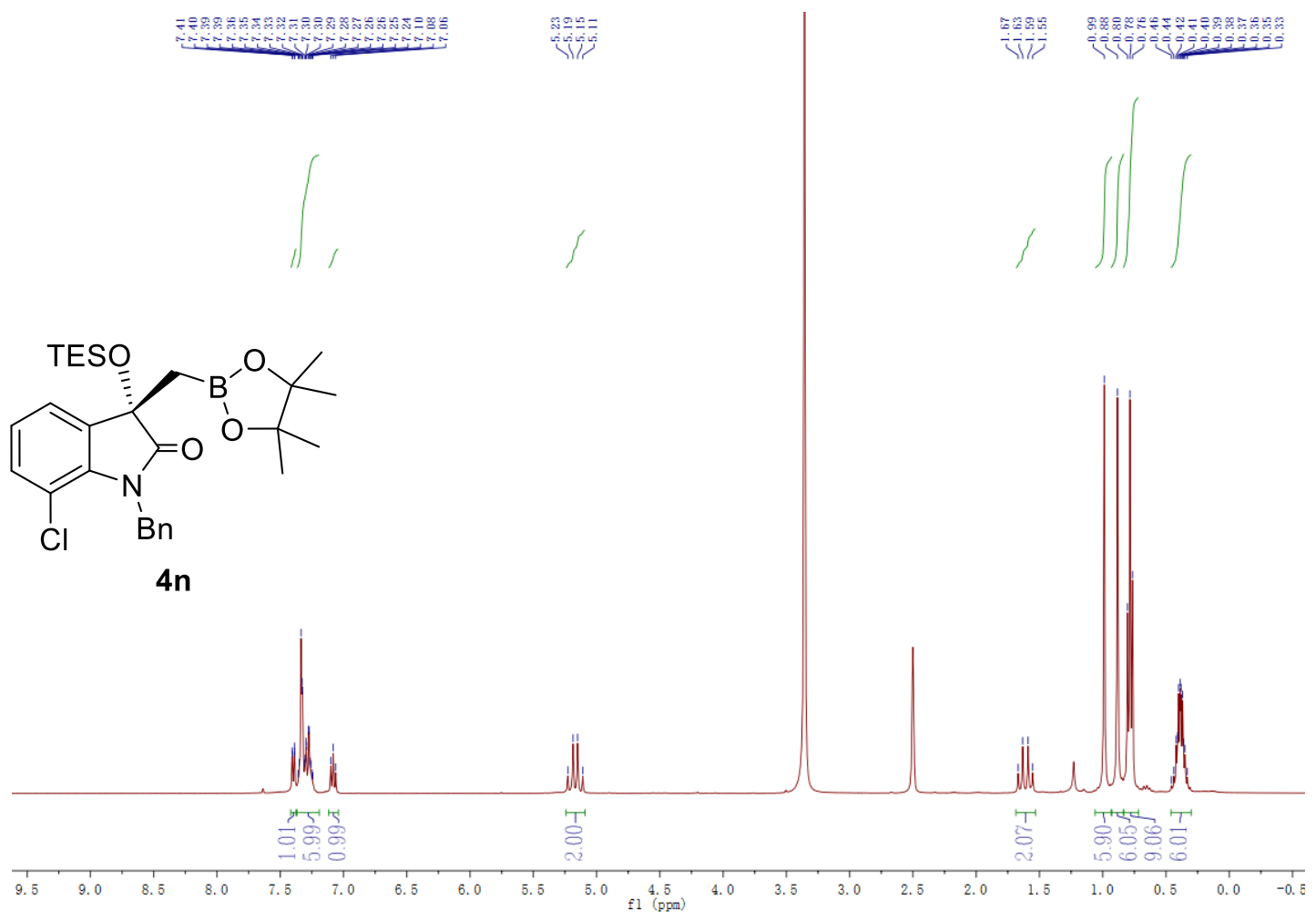

${ }^{13} \mathrm{C}$ NMR Spectrum of Compound 4n (101 MHz, DMSO-d6)

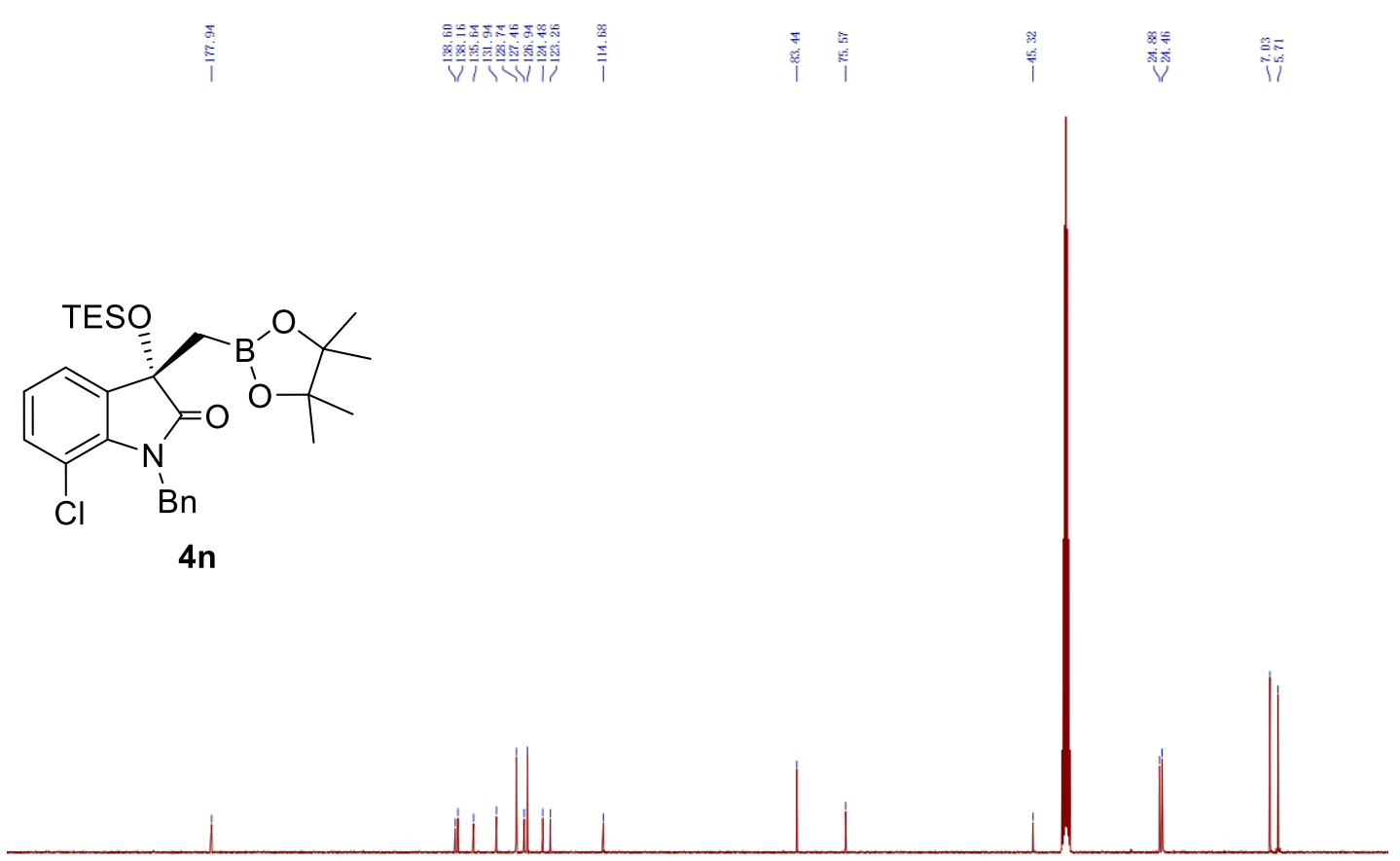

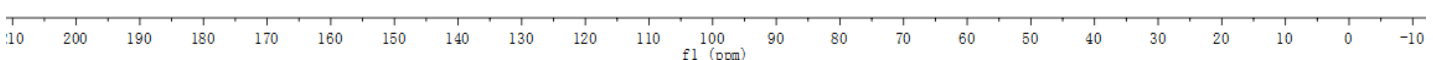


${ }^{11} \mathrm{~B}$ NMR Spectrum of Compound 4n (193 MHz, DMSO-d6)<smiles>COC1(CB2OC(C)(C)C(C)(C)O2)C(=O)N(Br)c2c(Cl)cccc21</smiles>

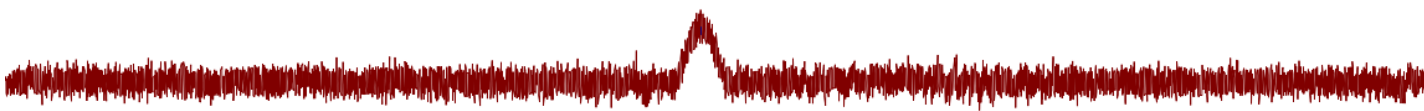


${ }^{1} \mathrm{H}$ NMR Spectrum of Compound 40 (400 MHz, DMSO-d6)

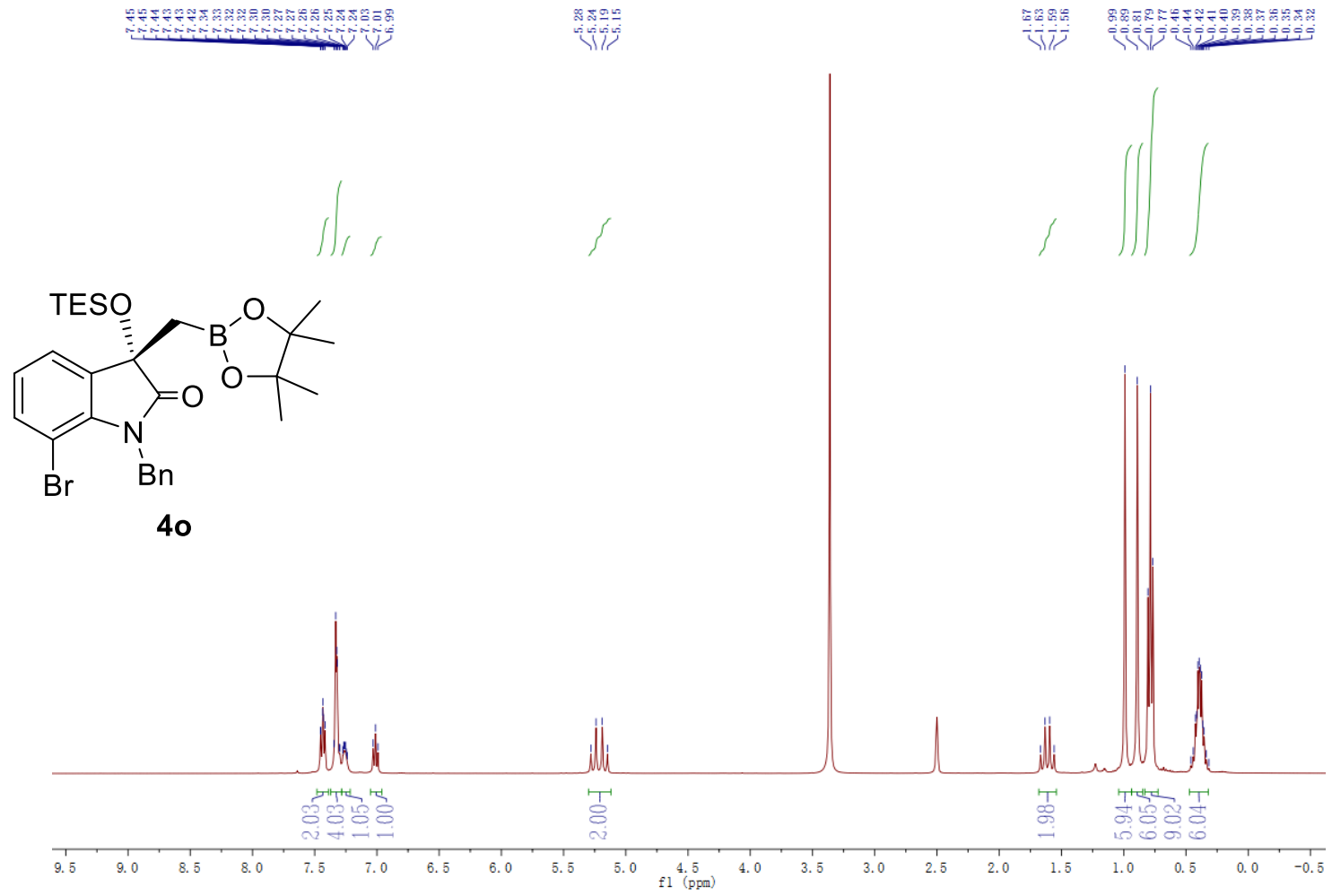

${ }^{13} \mathrm{C}$ NMR Spectrum of Compound 40 (101 MHz, DMSO-d6)

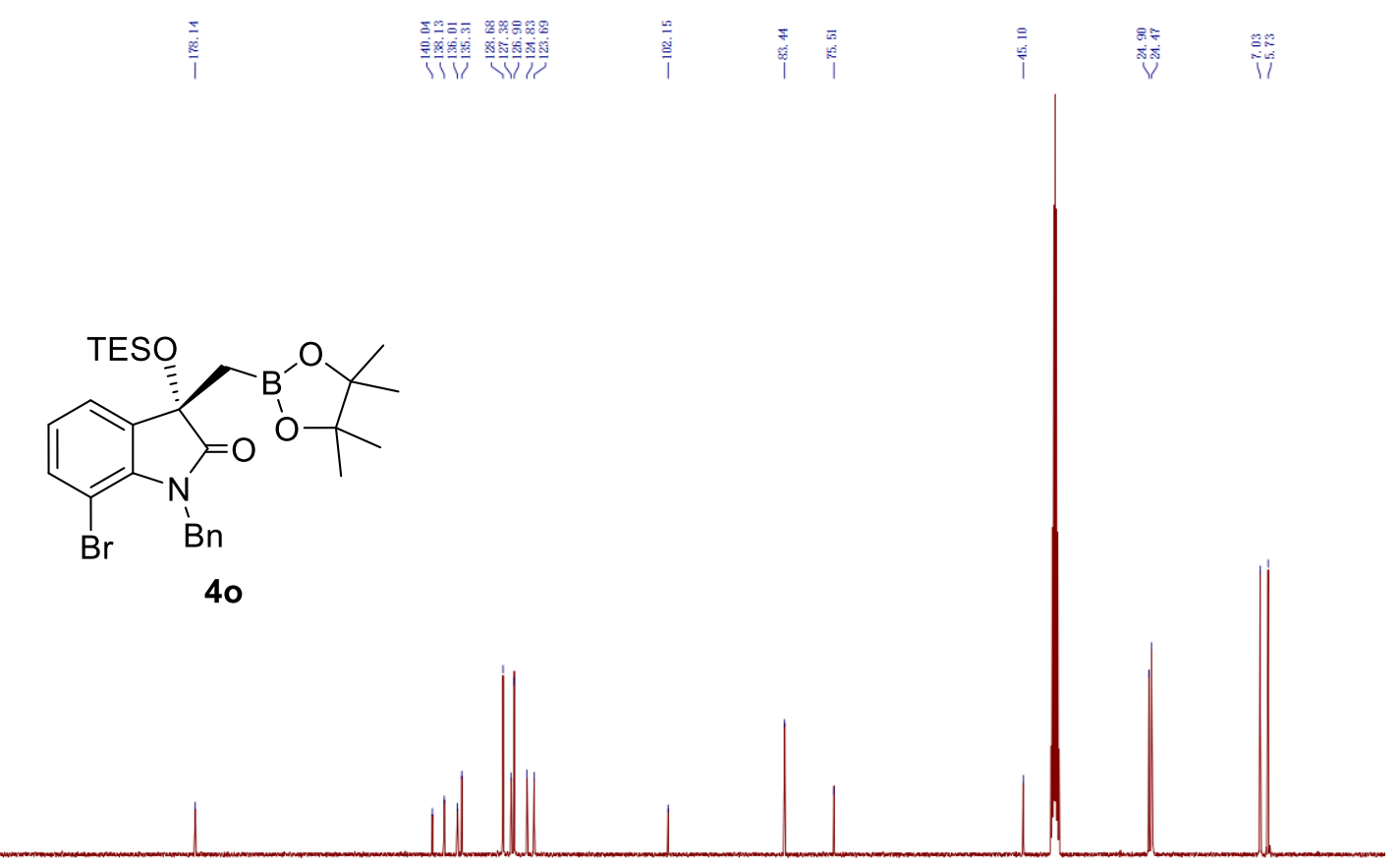

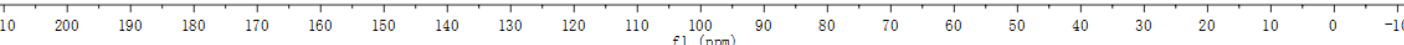


11B NMR Spectrum of Compound 40 (193 MHz, DMSO-d6)<smiles>COC[C@]1(CB2OC(C)(C)C(C)(C)O2)C(=O)Nc2c(Br)cccc21</smiles>

40

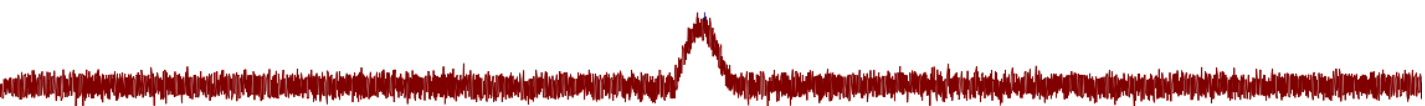


${ }^{1} \mathrm{H}$ NMR Spectrum of Compound $4 \mathrm{q}\left(400 \mathrm{MHz}, \mathrm{CDCl}_{3}\right)$

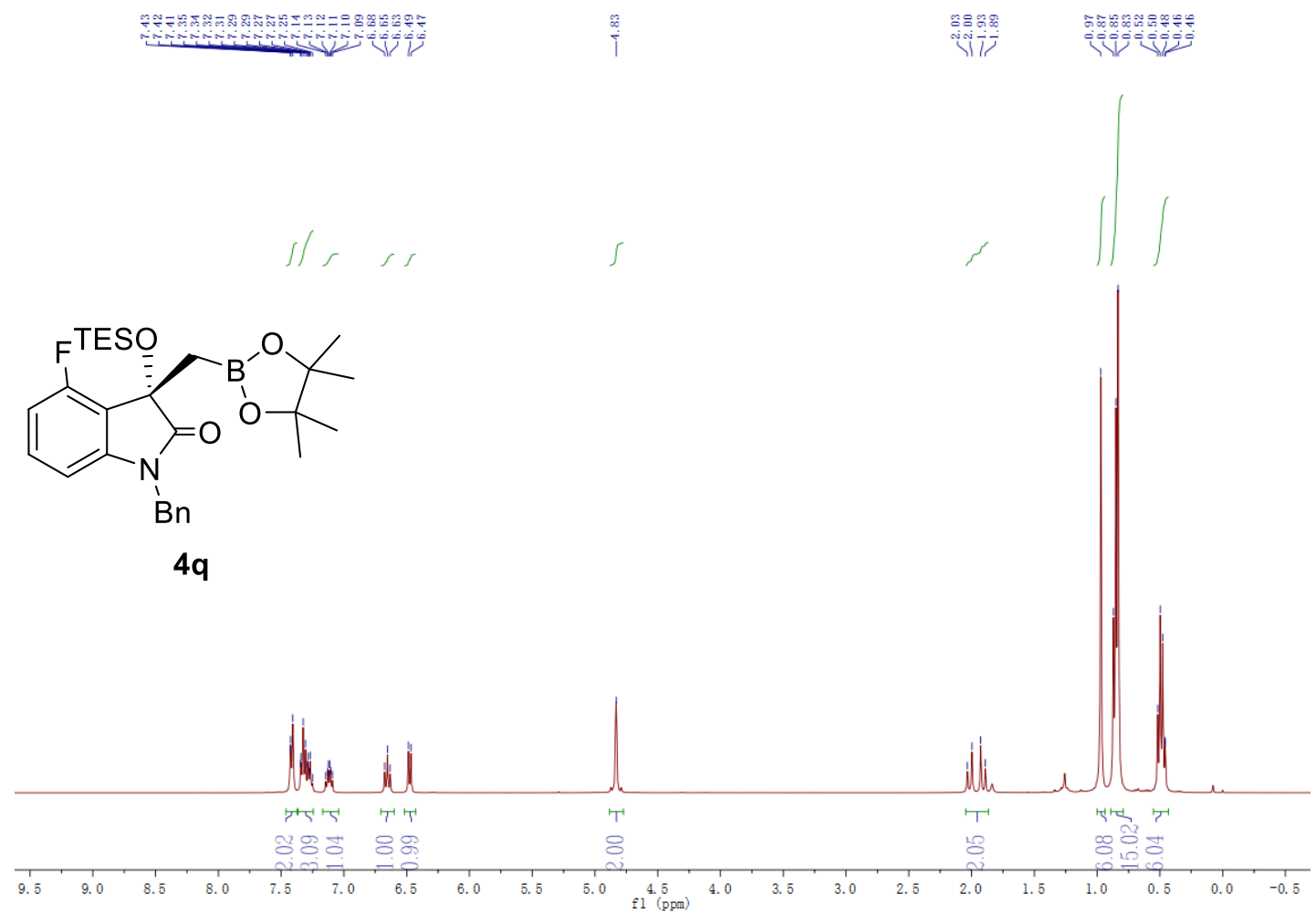

${ }^{13} \mathrm{C}$ NMR Spectrum of Compound $4 \mathrm{q}\left(151 \mathrm{MHz}, \mathrm{CDCl}_{3}\right)$

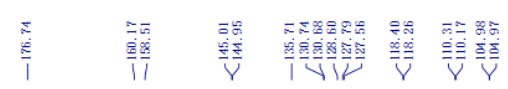
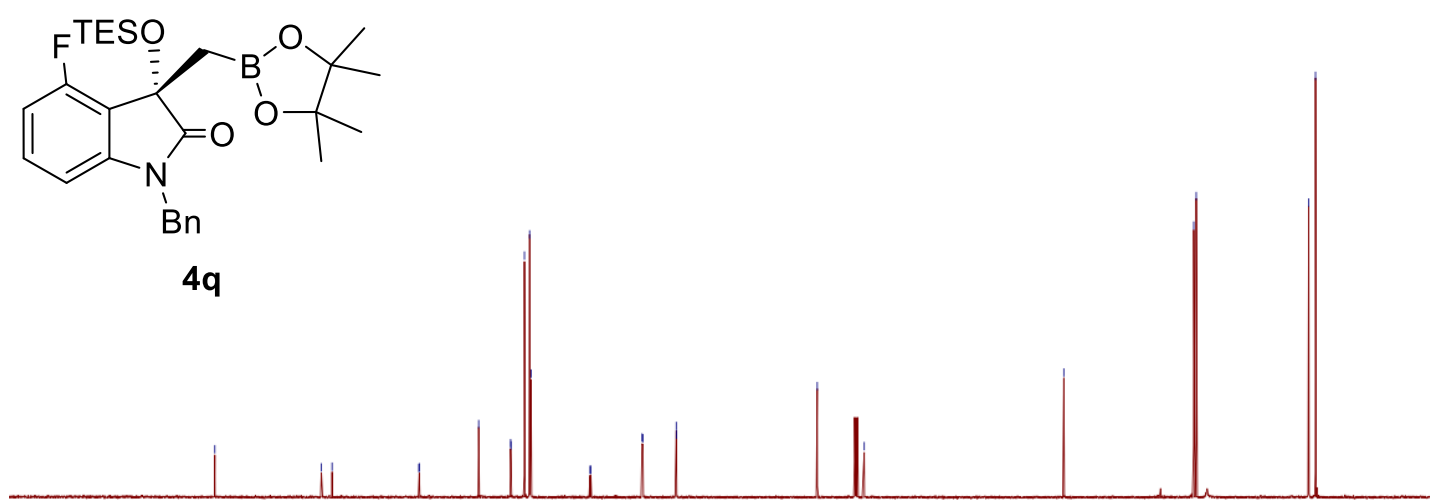

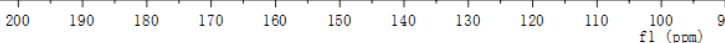


${ }^{11} \mathrm{~B}$ NMR Spectrum of Compound 4q (193 MHz, $\left.\mathrm{CDCl}_{3}\right)$

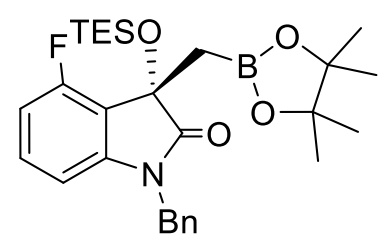

$4 q$
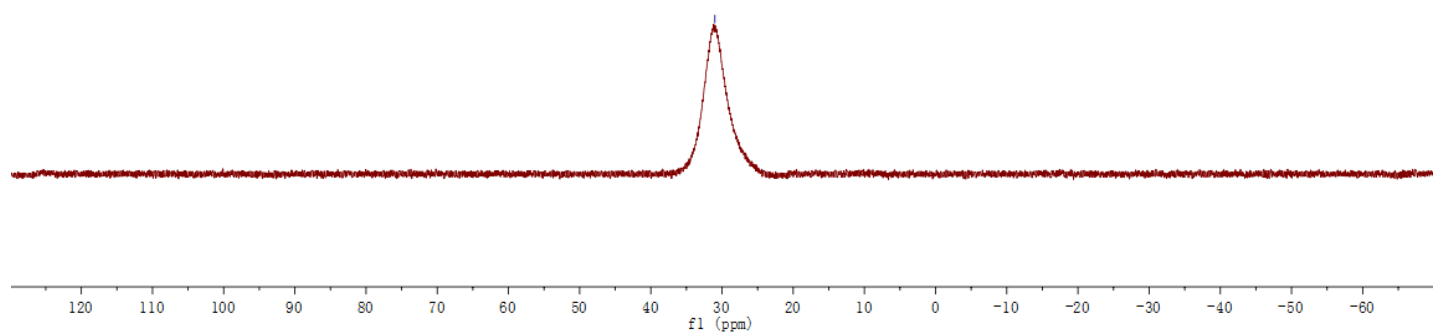

${ }^{19} \mathrm{~F}$ NMR Spectrum of Compound 4q (376 MHz, $\left.\mathrm{CDCl}_{3}\right)$<smiles>COC(=O)[C@@]1(CB2OC(C)(C)C(C)(C(C)(C)C)O2)C(=O)N([13CH3])c2cccc(F)c21</smiles>
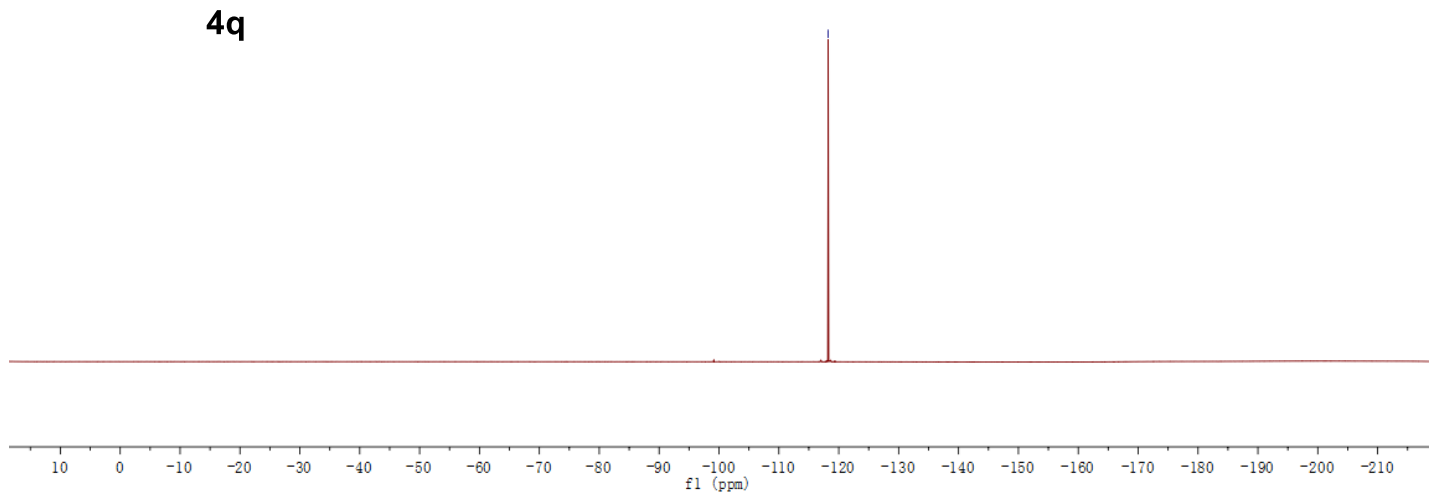
${ }^{1} \mathrm{H}$ NMR Spectrum of Compound $4 \mathrm{r}\left(400 \mathrm{MHz}, \mathrm{CDCl}_{3}\right)$

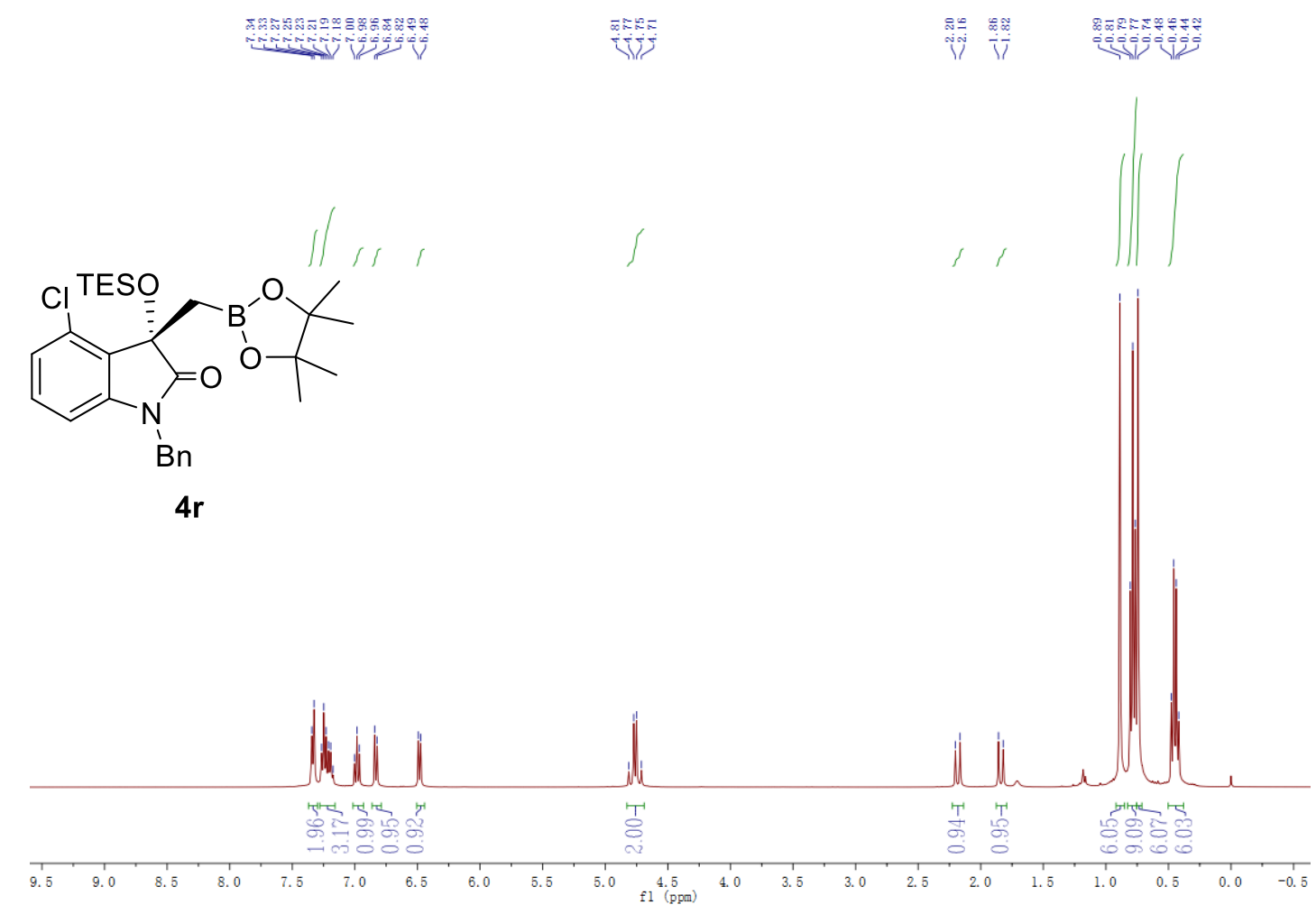

${ }^{13} \mathrm{C}$ NMR Spectrum of Compound $4 \mathrm{r}\left(151 \mathrm{MHz}, \mathrm{CDCl}_{3}\right)$
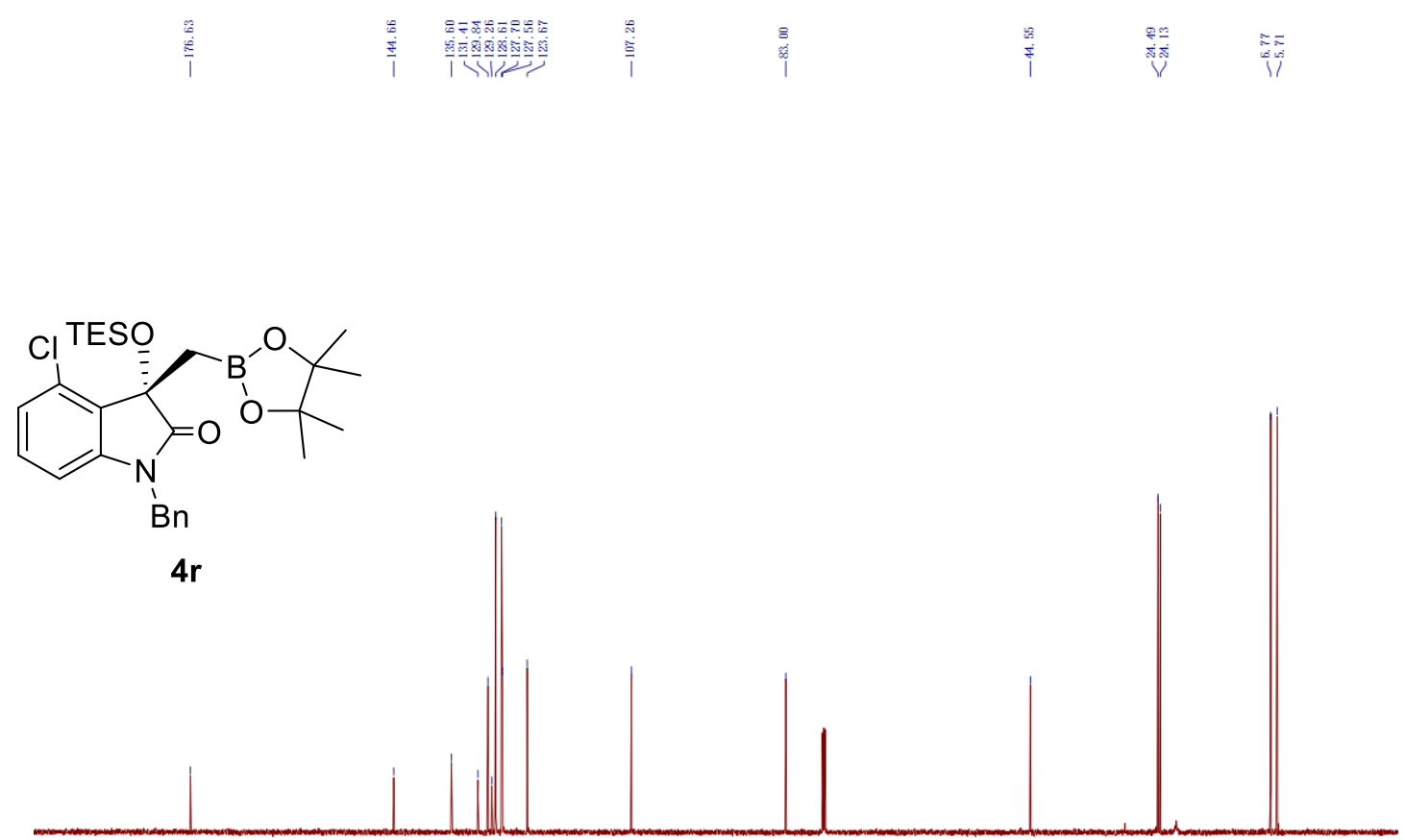
${ }^{11} \mathrm{~B}$ NMR Spectrum of Compound $4 \mathrm{r}\left(193 \mathrm{MHz}, \mathrm{CDCl}_{3}\right)$
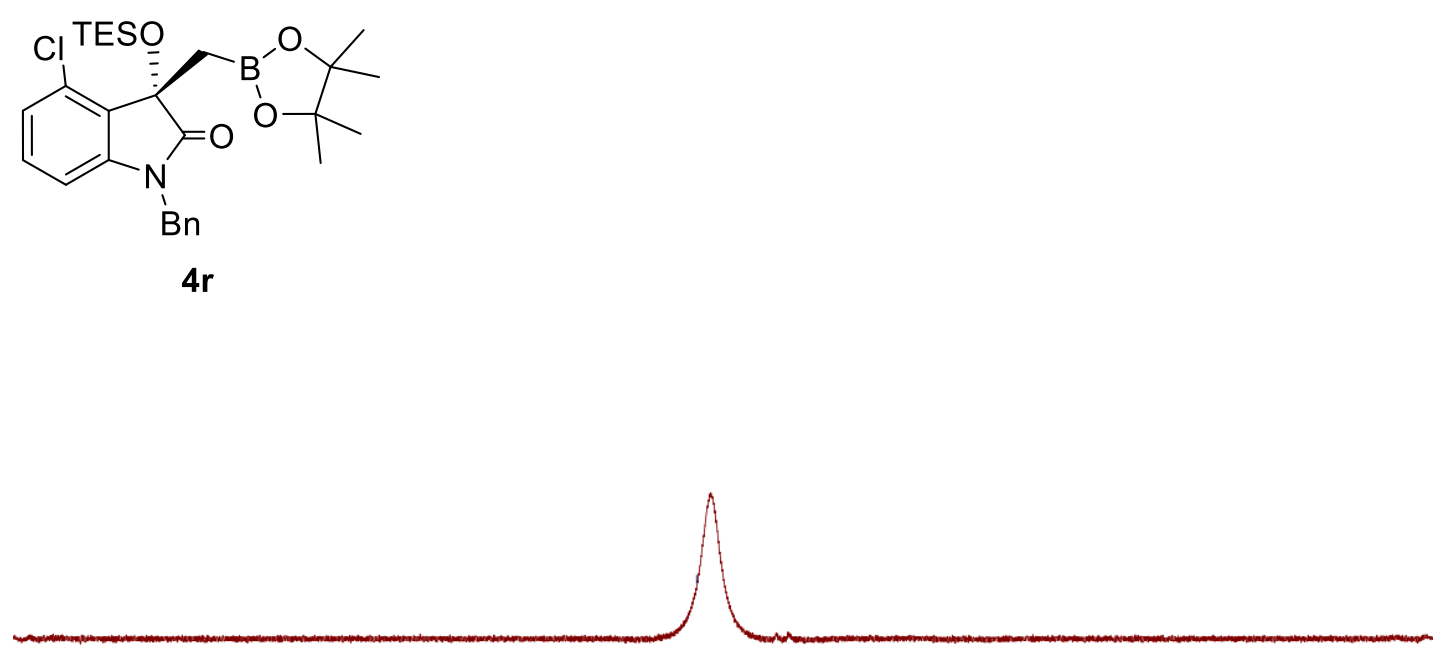
${ }^{1} \mathrm{H}$ NMR Spectrum of Compound $4 \mathrm{~s}\left(400 \mathrm{MHz}, \mathrm{CDCl}_{3}\right)$

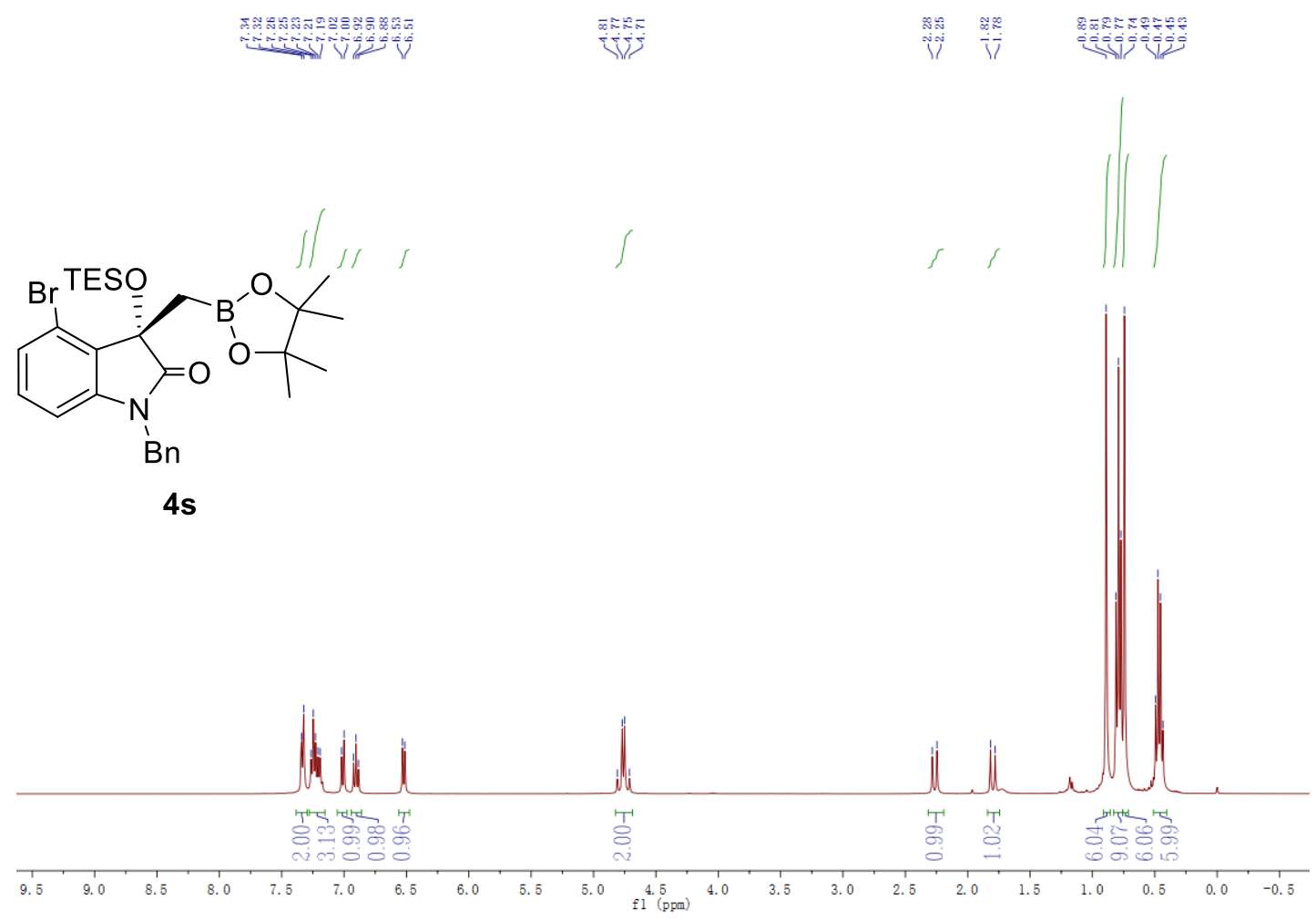

${ }^{13} \mathrm{C}$ NMR Spectrum of Compound 4s (151 MHz, $\left.\mathrm{CDCl}_{3}\right)$
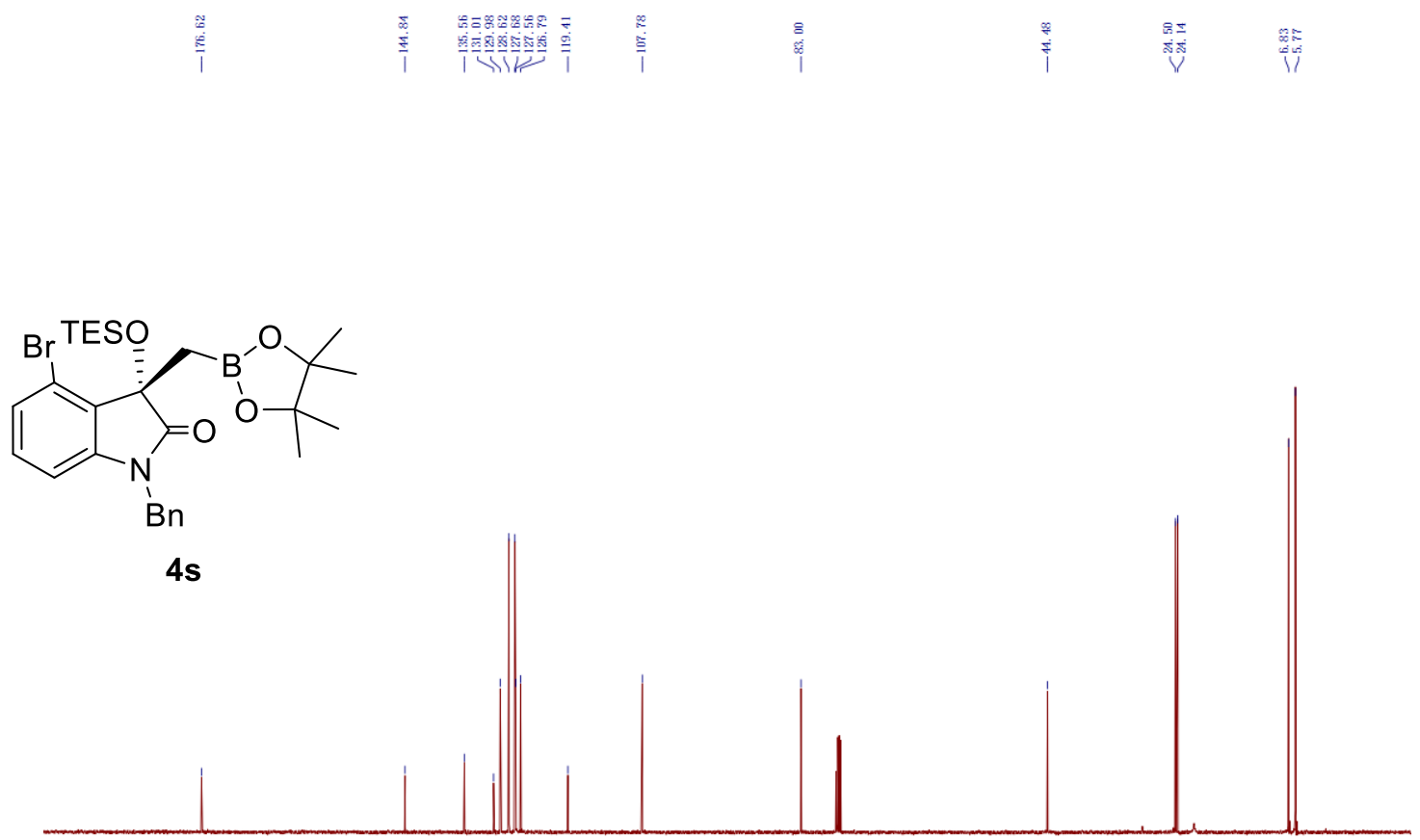
${ }^{11} \mathrm{~B}$ NMR Spectrum of Compound 4s (193 $\mathrm{MHz}, \mathrm{CDCl}_{3}$ )

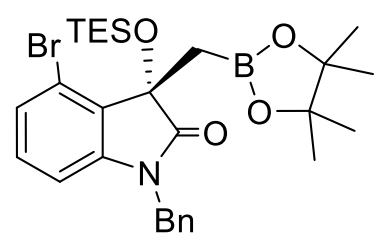

$4 s$

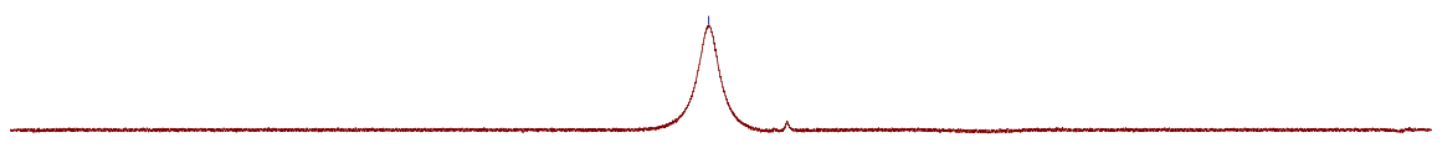


${ }^{1} \mathrm{H}$ NMR Spectrum of Compound 4t (400 MHz, DMSO-d6)

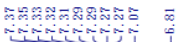

ำ

穴
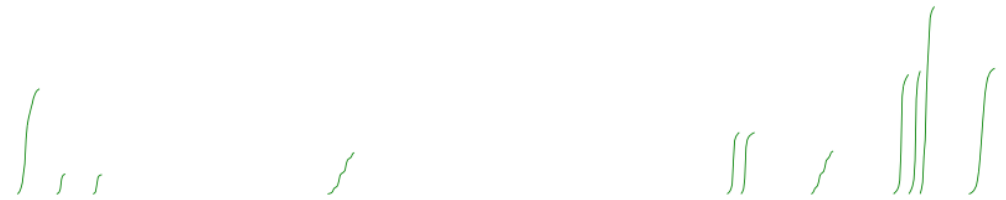<smiles>[Z14]#CN1C(=O)[C@@](CB2OC(C)(C)C(C)(C)O2)(OC(F)(F)F)c2cc(C)cc(C)c21</smiles>

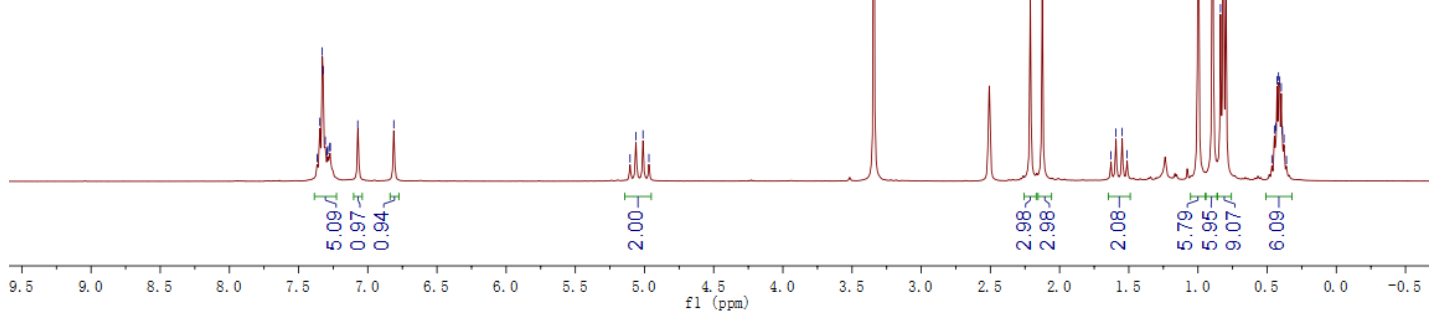

${ }^{13} \mathrm{C}$ NMR Spectrum of Compound 4t (151 MHz, DMSO-d6)
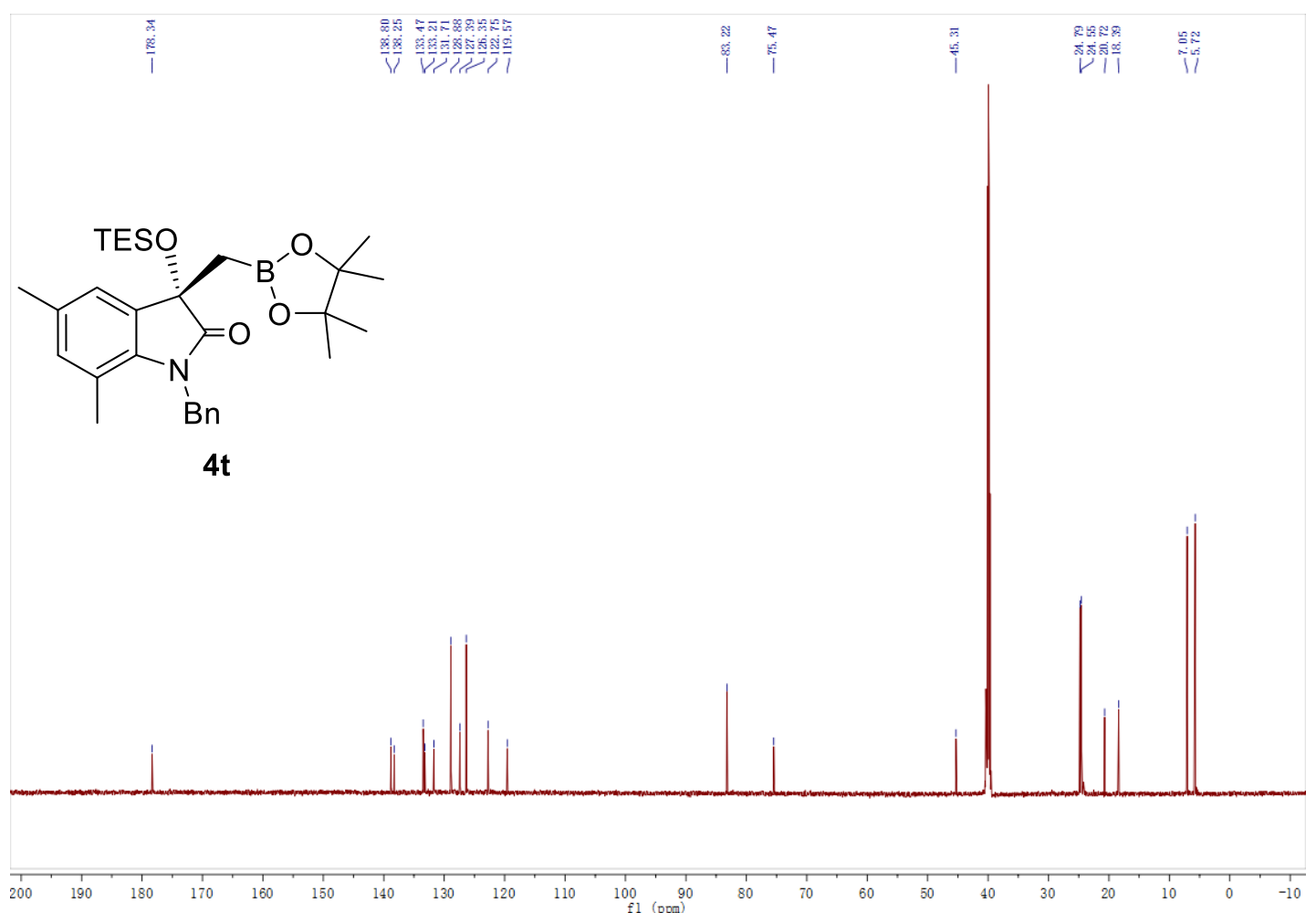
${ }^{11} \mathrm{~B}$ NMR Spectrum of Compound 4t (193 MHz, DMSO-d6)<smiles>COC1(CB2OC(C)(C)C(C)(C)O2)C(=O)Nc2c(C)cc(C)cc21</smiles>

$4 t$

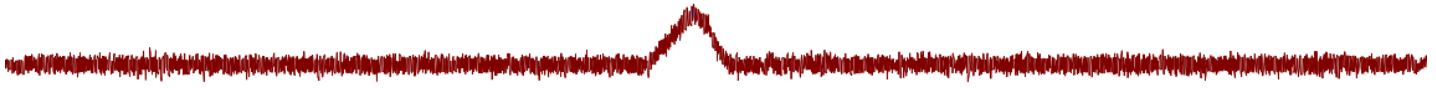


${ }^{1} \mathrm{H}$ NMR Spectrum of Compound $4 \mathrm{u}\left(400 \mathrm{MHz}, \mathrm{CDCl}_{3}\right)$

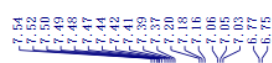

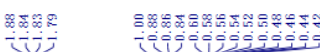

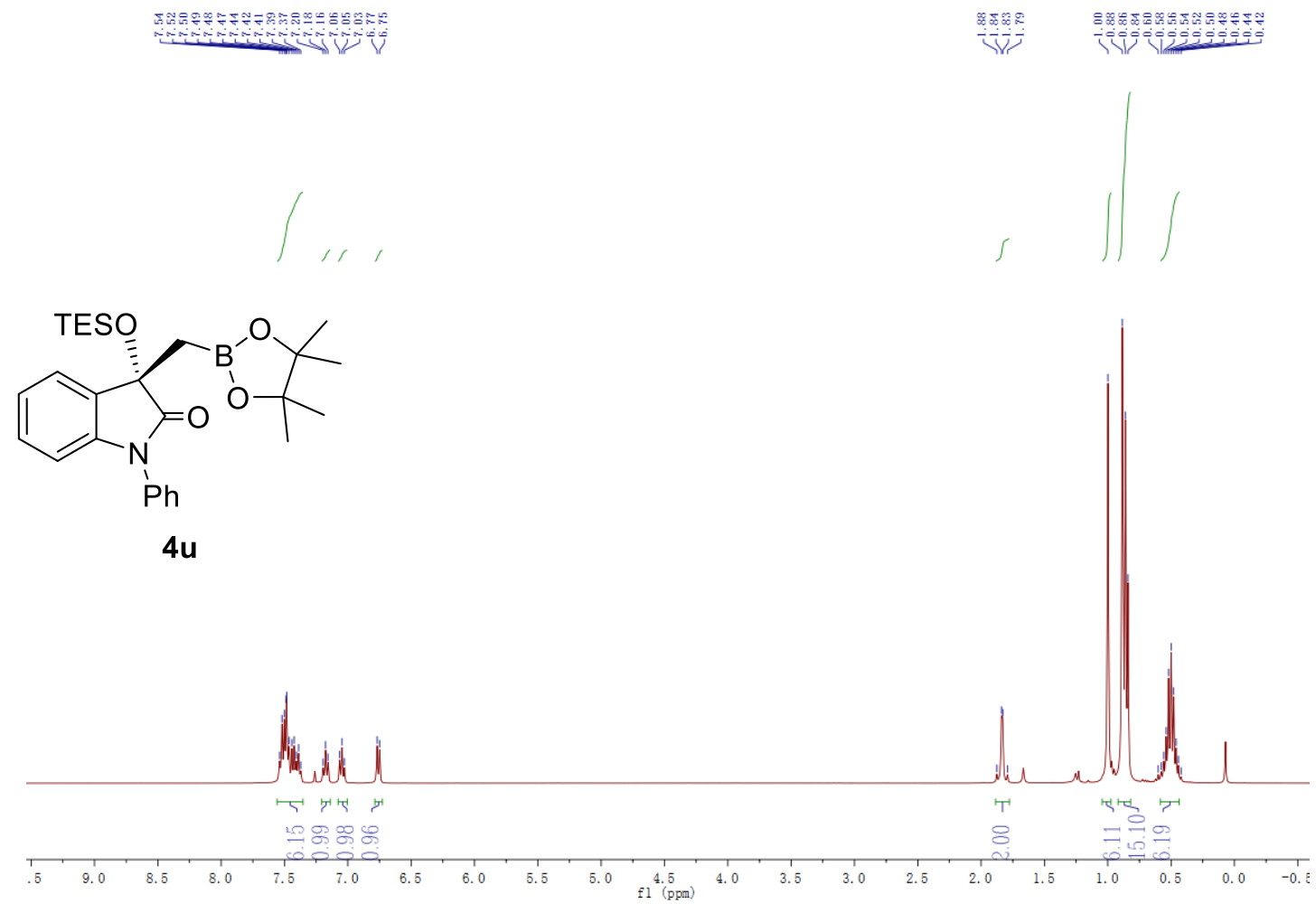

${ }^{13} \mathrm{C}$ NMR Spectrum of Compound $4 \mathrm{u}\left(101 \mathrm{MHz}, \mathrm{CDCl}_{3}\right)$
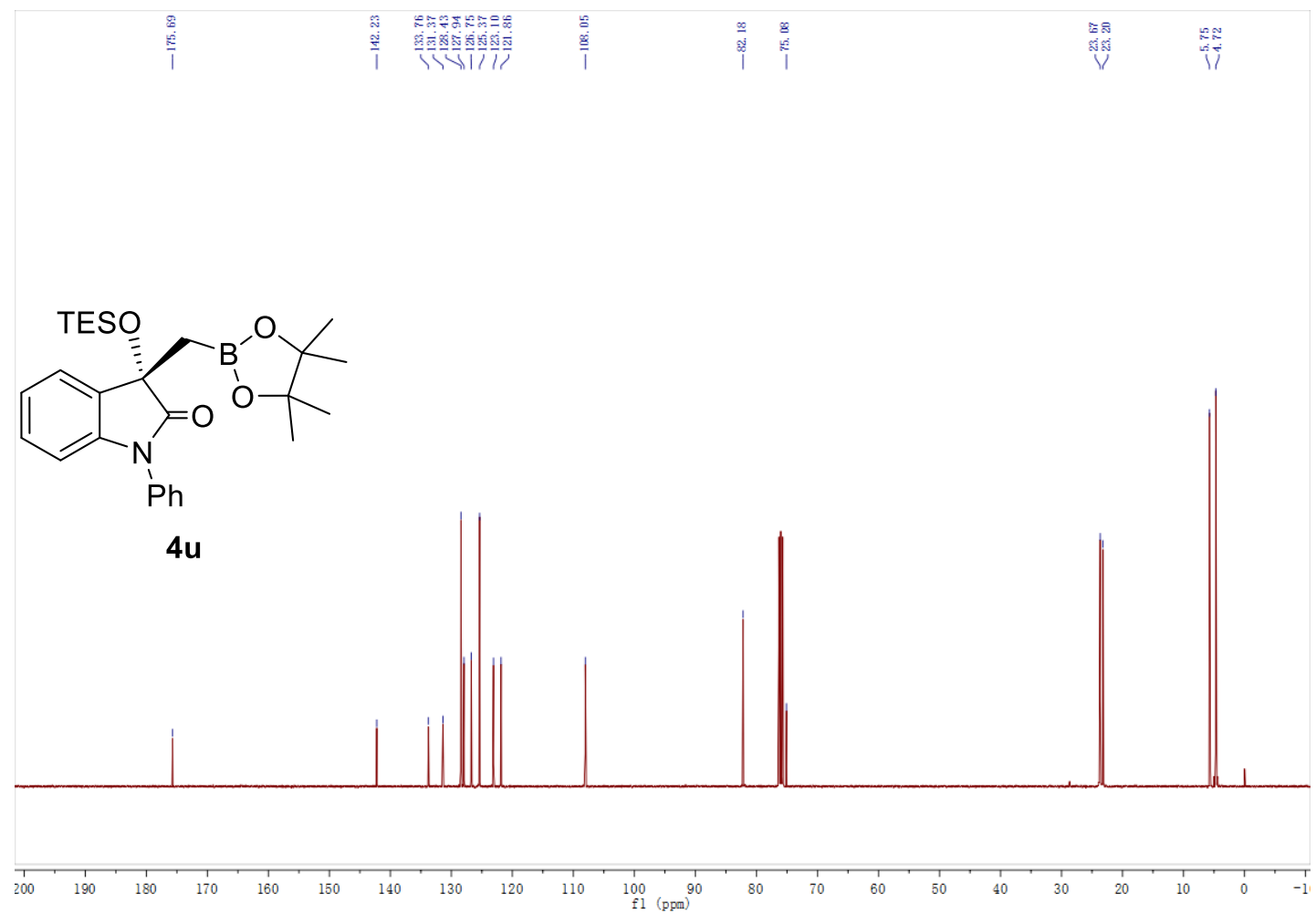
${ }^{11} \mathrm{~B}$ NMR Spectrum of Compound 4u (193 $\left.\mathrm{MHz}, \mathrm{CDCl}_{3}\right)$
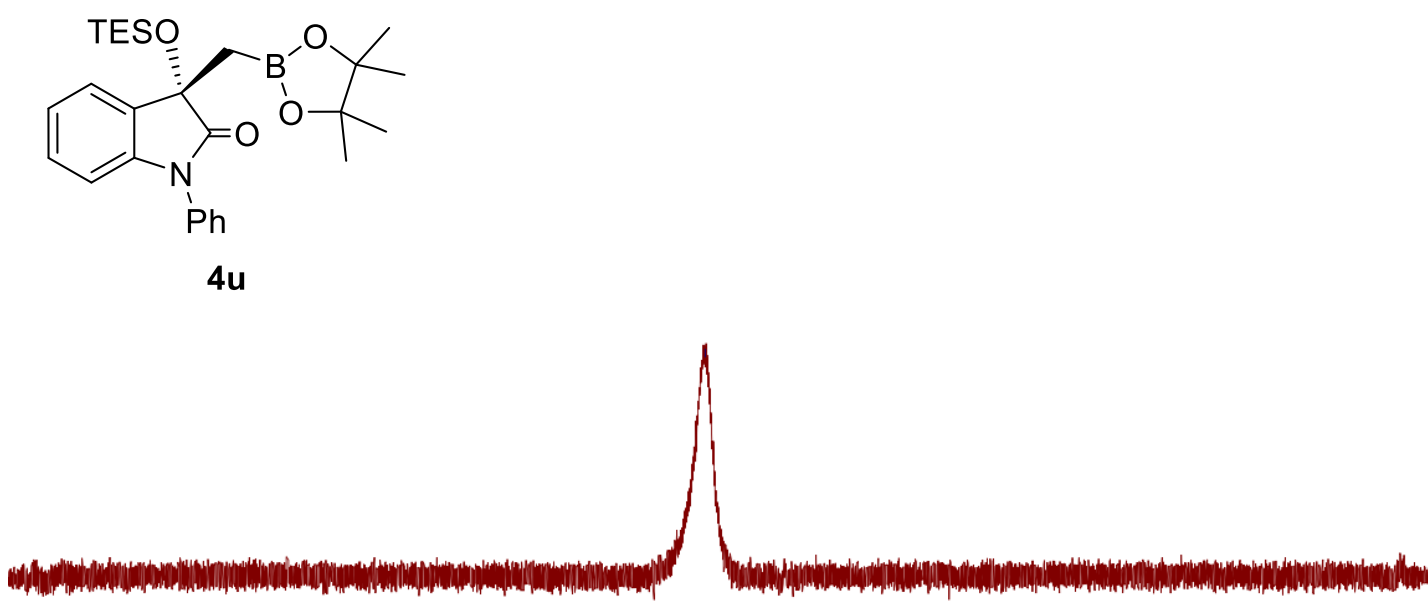
${ }^{1} \mathrm{H}$ NMR Spectrum of Compound $4 \mathrm{v}\left(400 \mathrm{MHz}, \mathrm{CDCl}_{3}\right)$

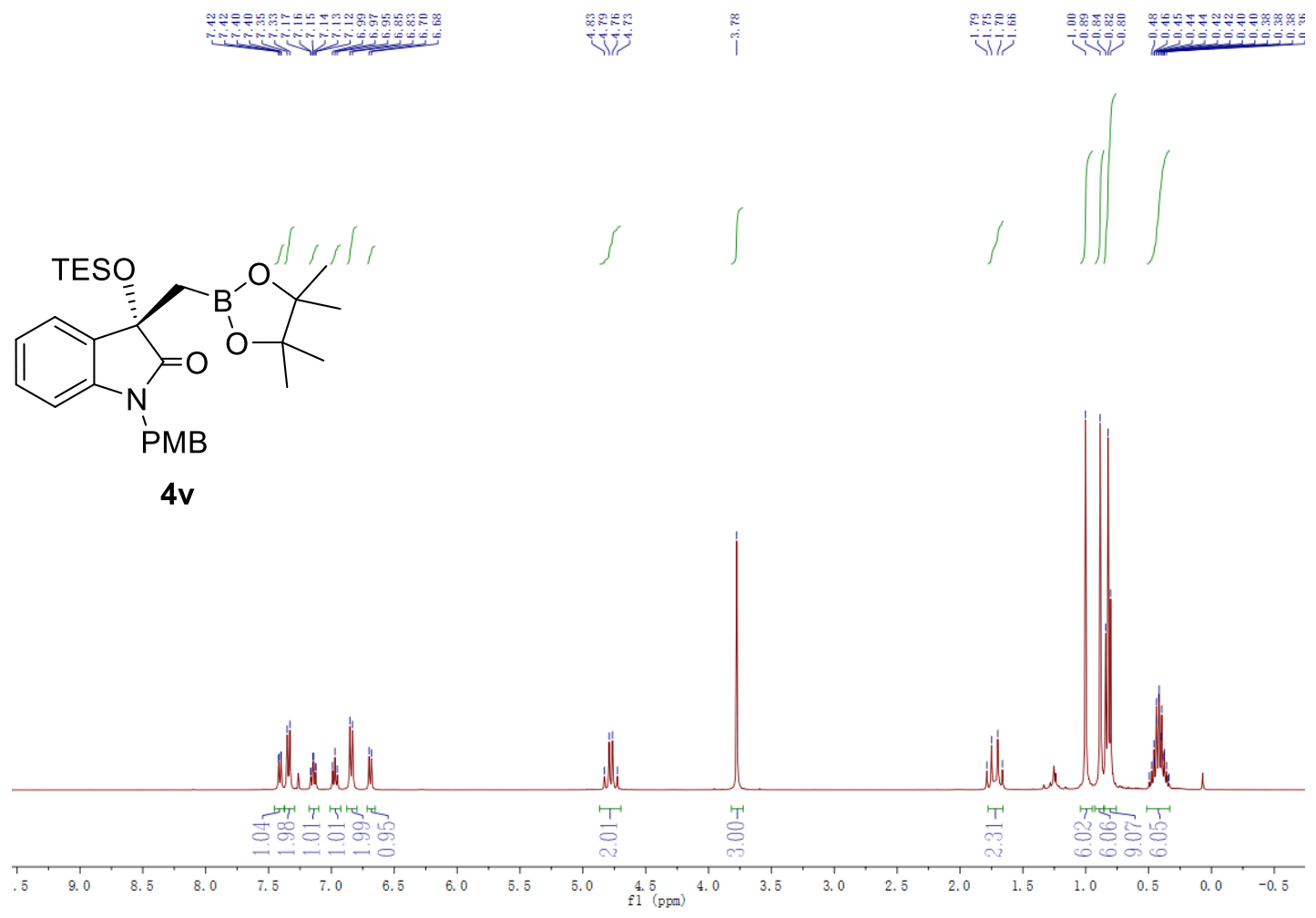

${ }^{13} \mathrm{C}$ NMR Spectrum of Compound $4 \mathrm{v}\left(151 \mathrm{MHz}, \mathrm{CDCl}_{3}\right)$

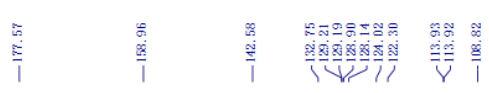

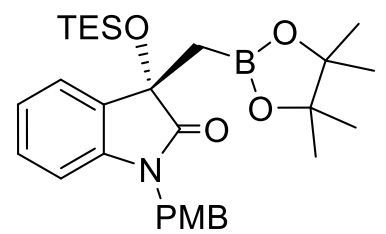

PMB

$4 v$
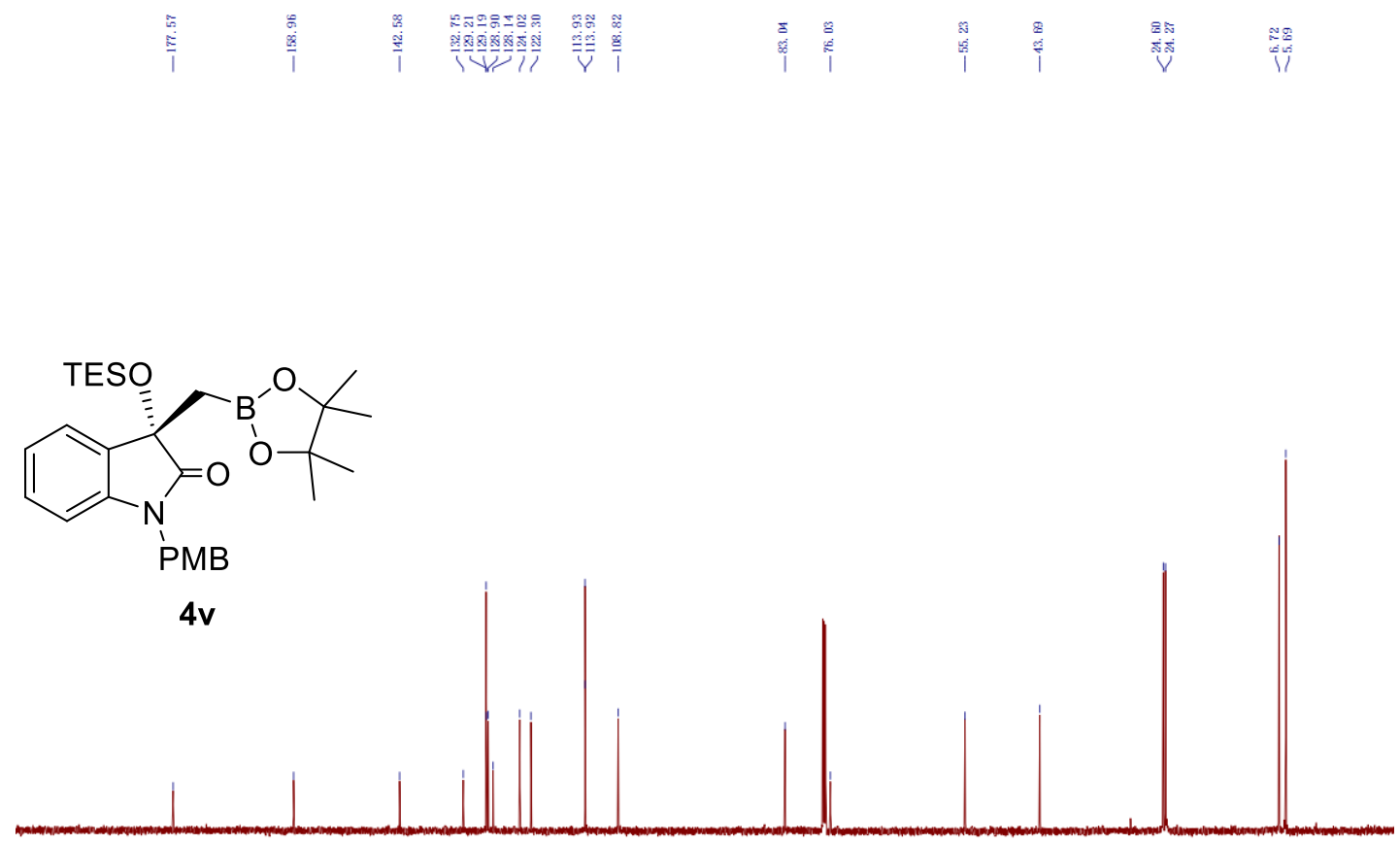

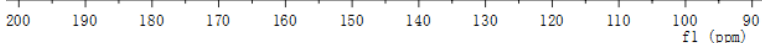


${ }^{11} \mathrm{~B}$ NMR Spectrum of Compound 4v (193 $\left.\mathrm{MHz}^{\mathrm{CDCl}} \mathrm{C}_{3}\right)$<smiles>CC(C)(C)O[C@]1(CB2OC(C)(C)C(C)(C)O2)C(=O)N([13CH3])c2ccccc21</smiles>

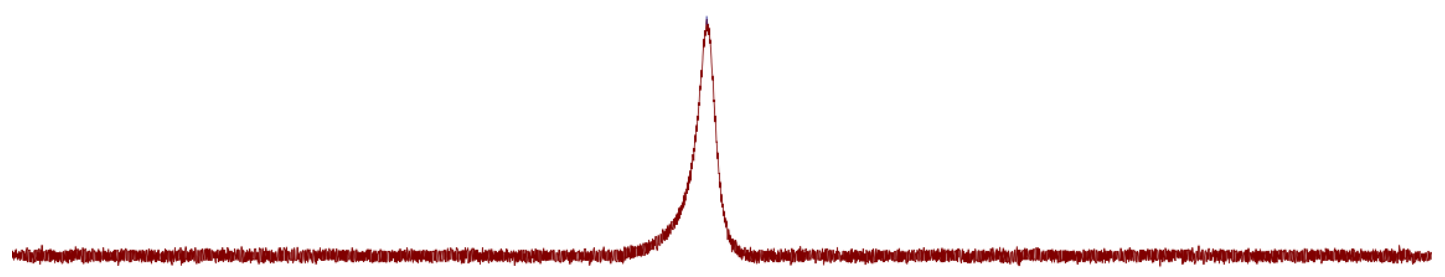


${ }^{1} \mathrm{H}$ NMR Spectrum of Compound $4 \mathrm{w}\left(400 \mathrm{MHz}, \mathrm{CDCl}_{3}\right)$

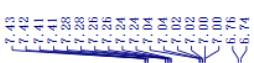

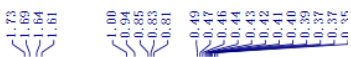

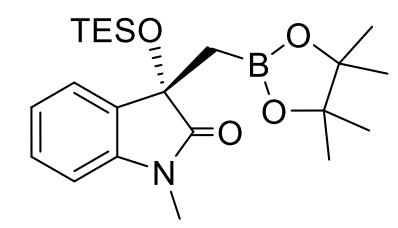

$4 w$

M
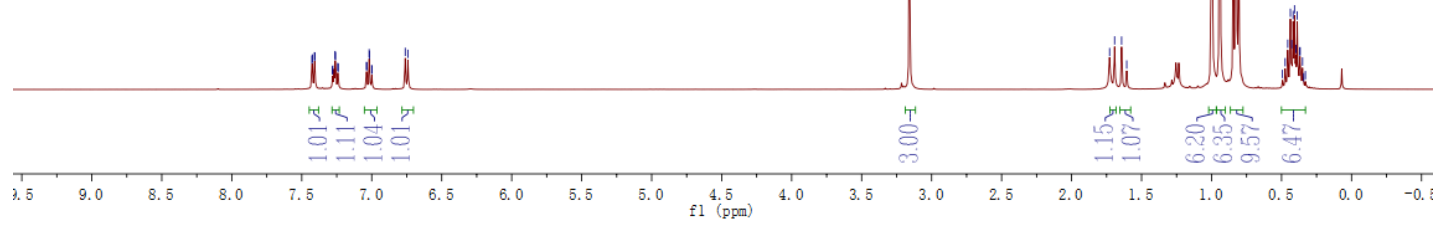

${ }^{13} \mathrm{C}$ NMR Spectrum of Compound 4w (151 $\left.\mathrm{MHz}, \mathrm{CDCl}_{3}\right)$

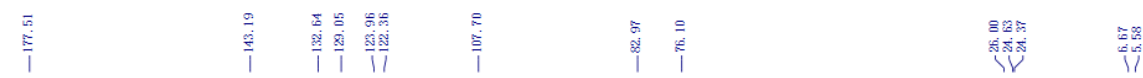

$\sum_{N=0}^{T E S O}=$

$4 w$

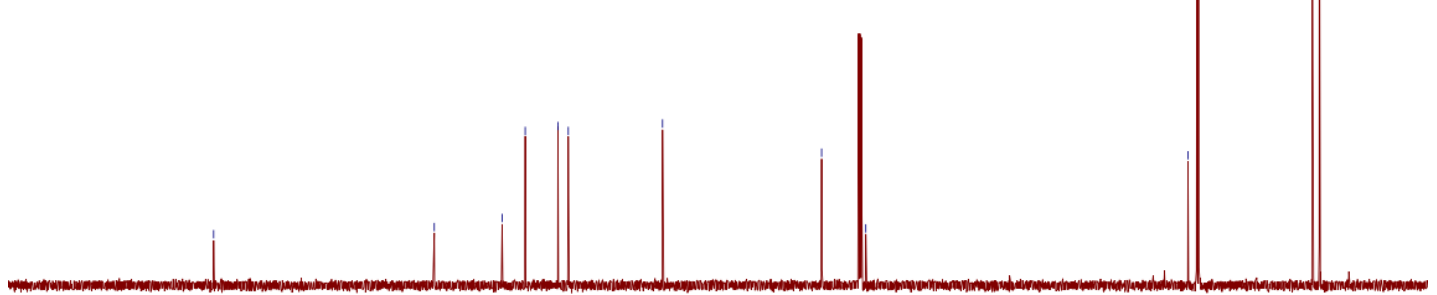

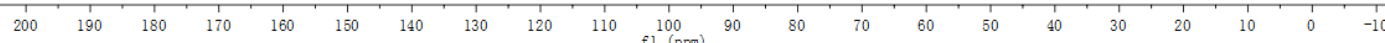


${ }^{11} \mathrm{~B}$ NMR Spectrum of Compound 4w (193 MHz, $\left.\mathrm{CDCl}_{3}\right)$
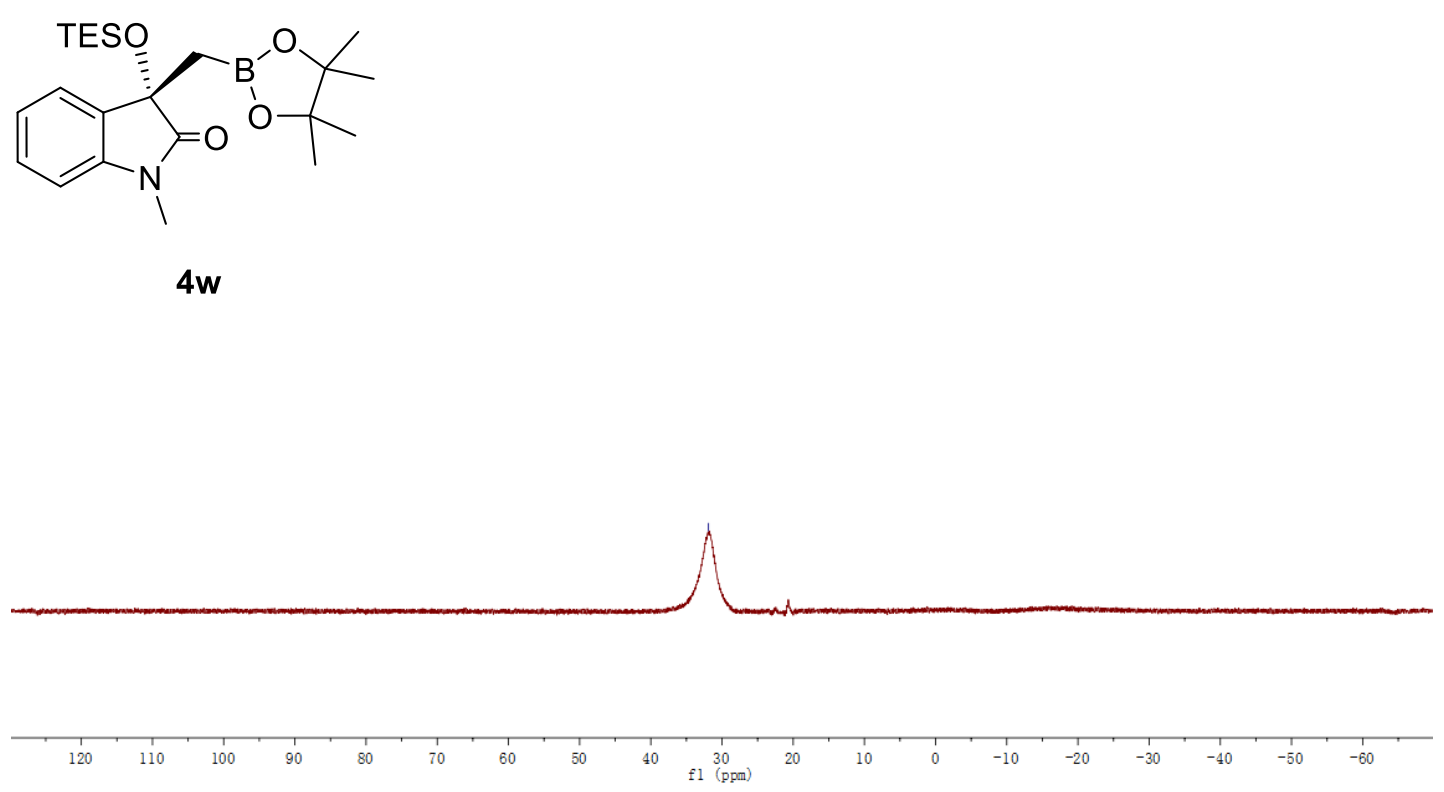
${ }^{1} \mathrm{H}$ NMR Spectrum of Compound $6 \mathrm{a}\left(400 \mathrm{MHz}, \mathrm{CDCl}_{3}\right)$

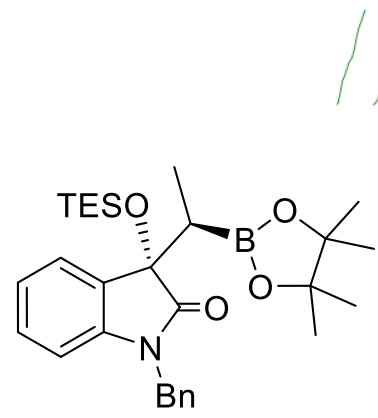

$6 a$

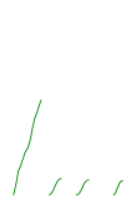

Q

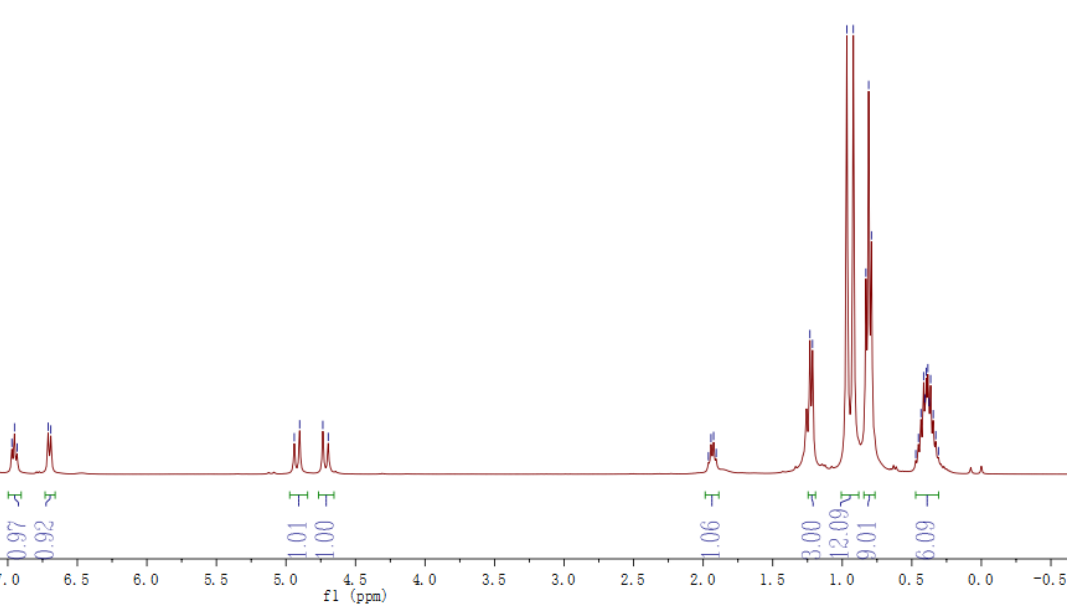

${ }^{13} \mathrm{C}$ NMR Spectrum of Compound $6 \mathrm{a}\left(151 \mathrm{MHz}, \mathrm{CDCl}_{3}\right)$

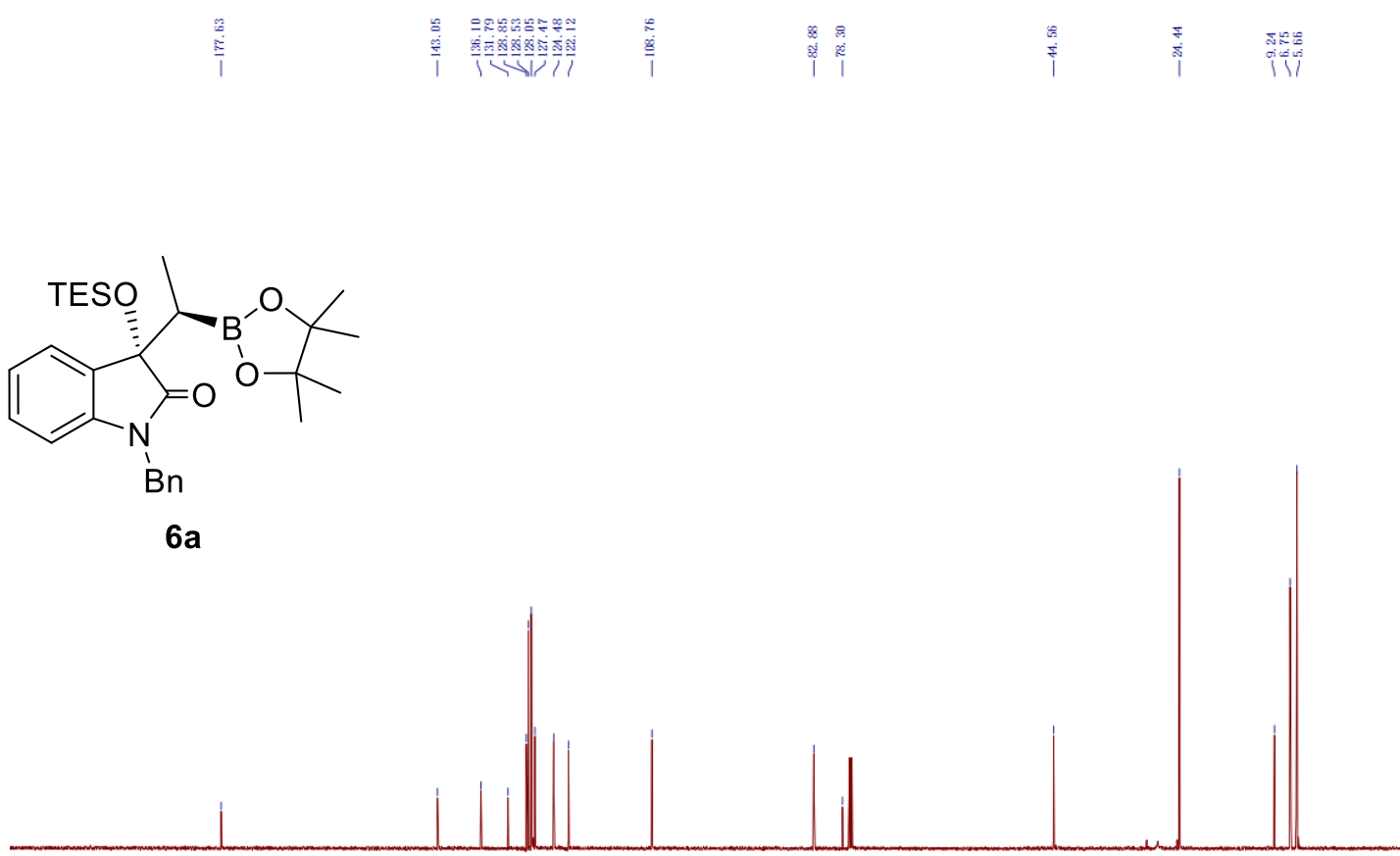

$10 \quad 200 \quad 190$

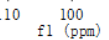


${ }^{11} \mathrm{~B}$ NMR Spectrum of Compound $6 \mathrm{a}\left(193 \mathrm{MHz}, \mathrm{CDCl}_{3}\right)$

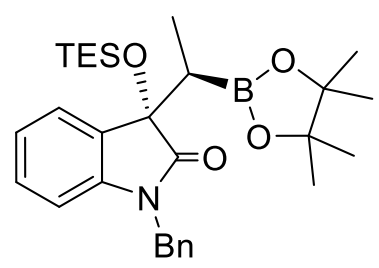

$6 a$

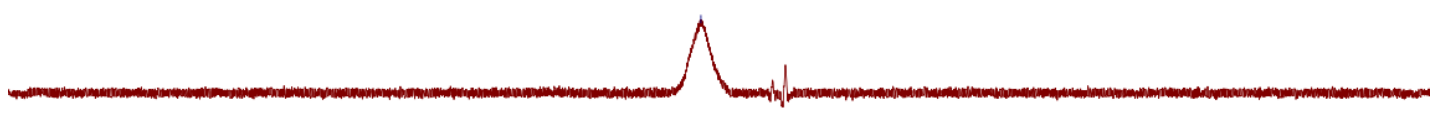


${ }^{1} \mathrm{H}$ NMR Spectrum of Compound $6 \mathrm{~b}\left(400 \mathrm{MHz}, \mathrm{CDCl}_{3}\right)$

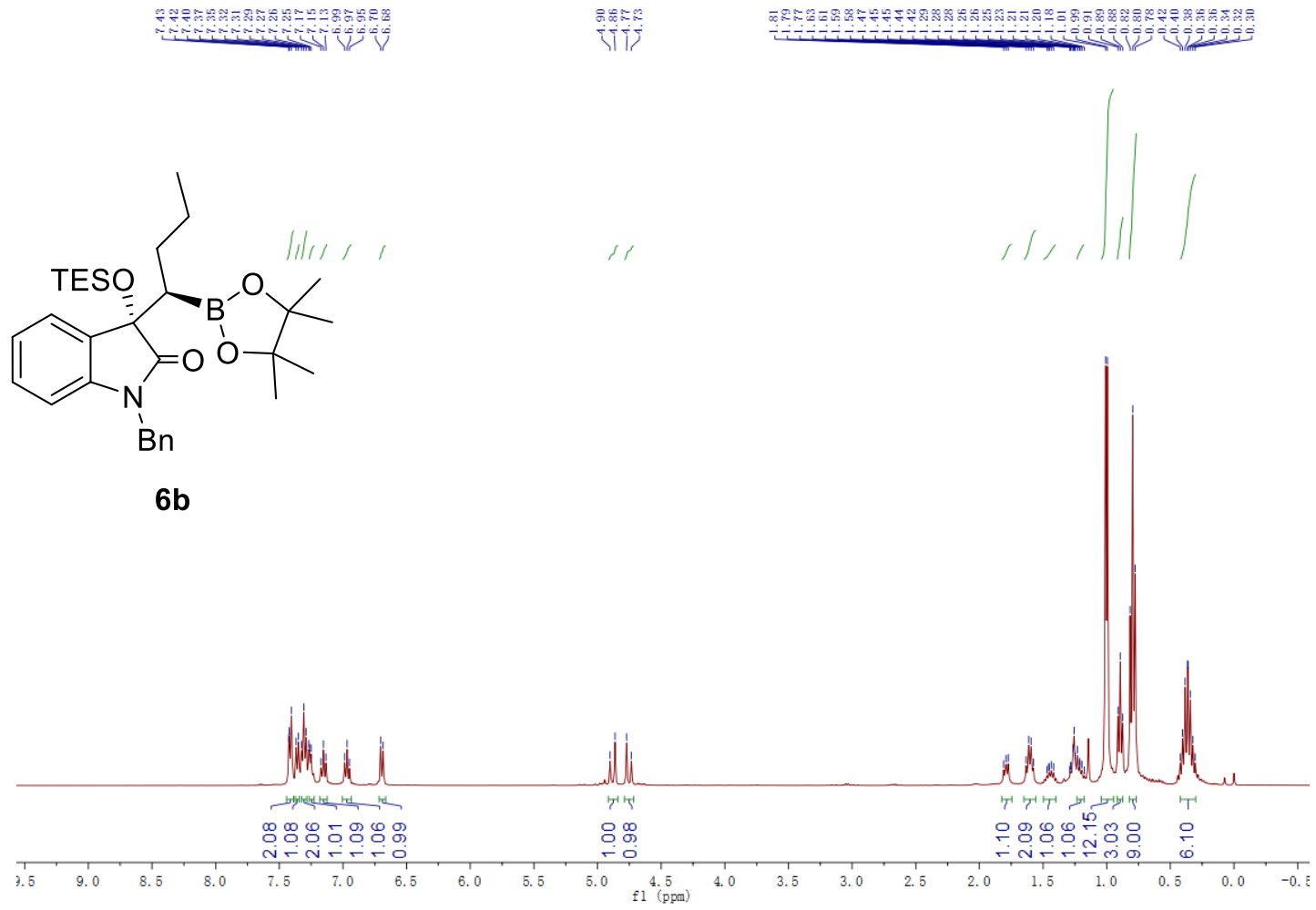

${ }^{13} \mathrm{C}$ NMR Spectrum of Compound $6 \mathrm{~b}\left(101 \mathrm{MHz}, \mathrm{CDCl}_{3}\right)$
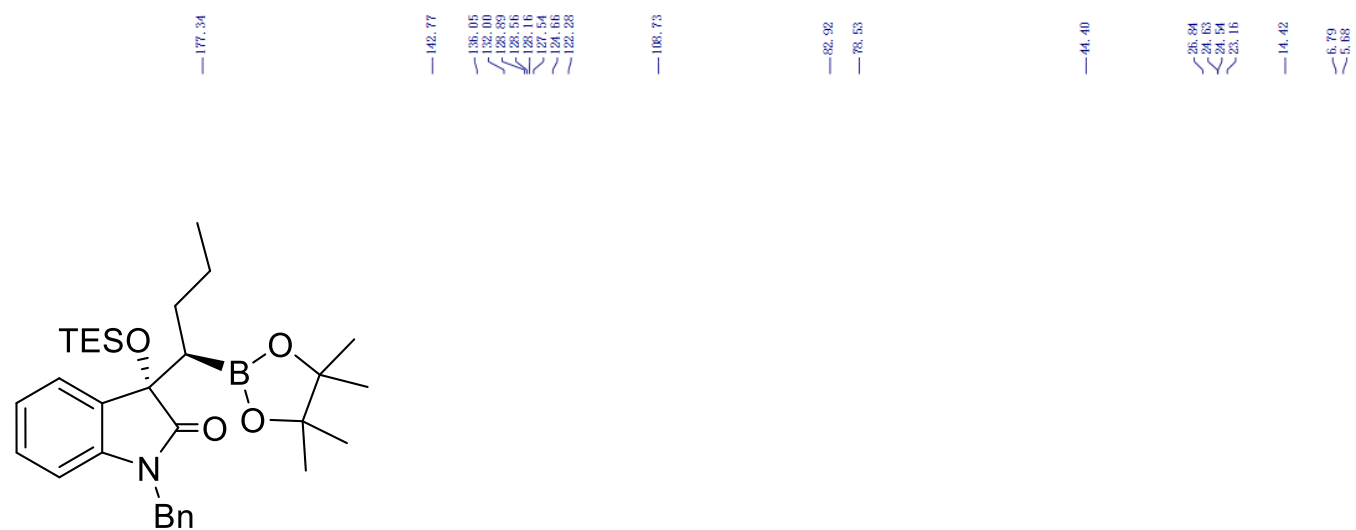

$6 b$
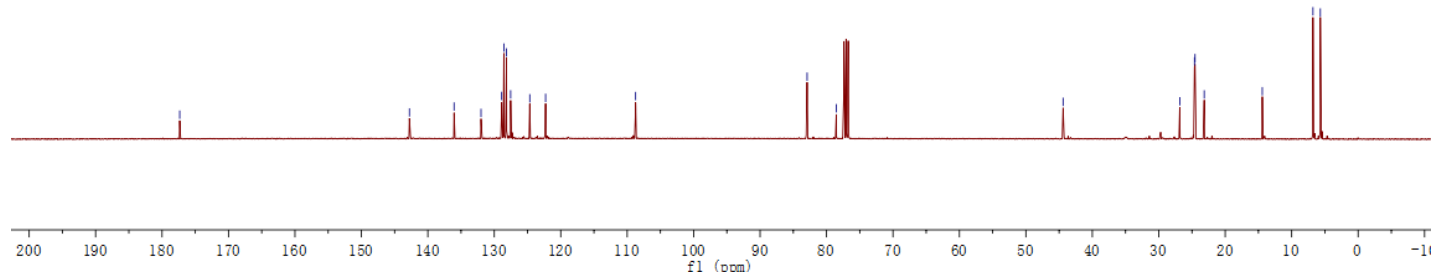
${ }^{11} \mathrm{~B}$ NMR Spectrum of Compound $6 \mathrm{~b}\left(193 \mathrm{MHz}, \mathrm{CDCl}_{3}\right)$

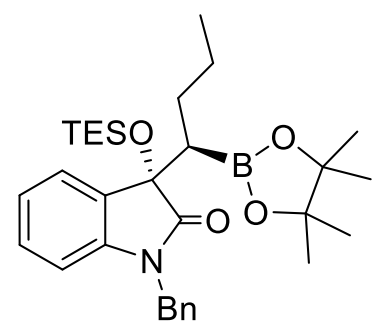

6b

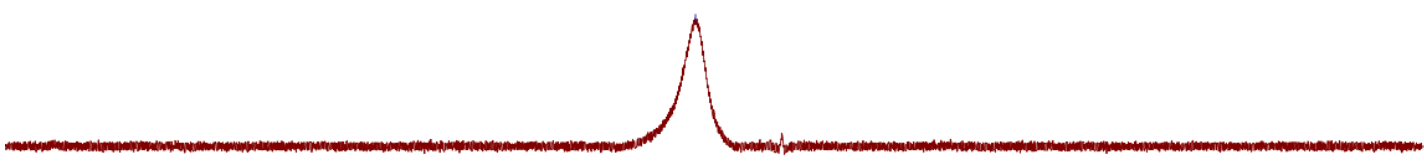


${ }^{1} \mathrm{H}$ NMR Spectrum of Compound $6 \mathrm{c}\left(400 \mathrm{MHz}, \mathrm{CDCl}_{3}\right)$

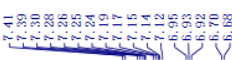

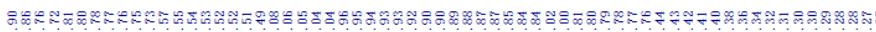

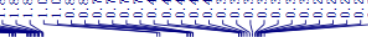<smiles>CC1(C)OB(C(CCc2ccccc2)C2C(=O)N(Cc3ccccc3)c3ccccc32)OC1(C)C</smiles>

$6 c$

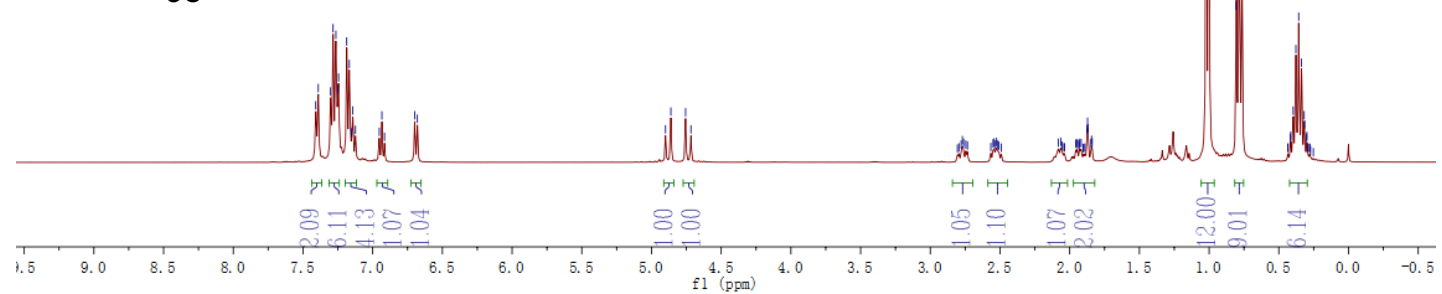

${ }^{13} \mathrm{C}$ NMR Spectrum of Compound $6 \mathrm{c}\left(101 \mathrm{MHz}, \mathrm{CDCl}_{3}\right)$

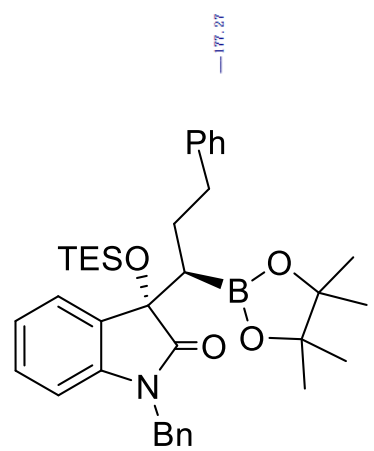

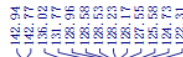

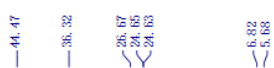

6c

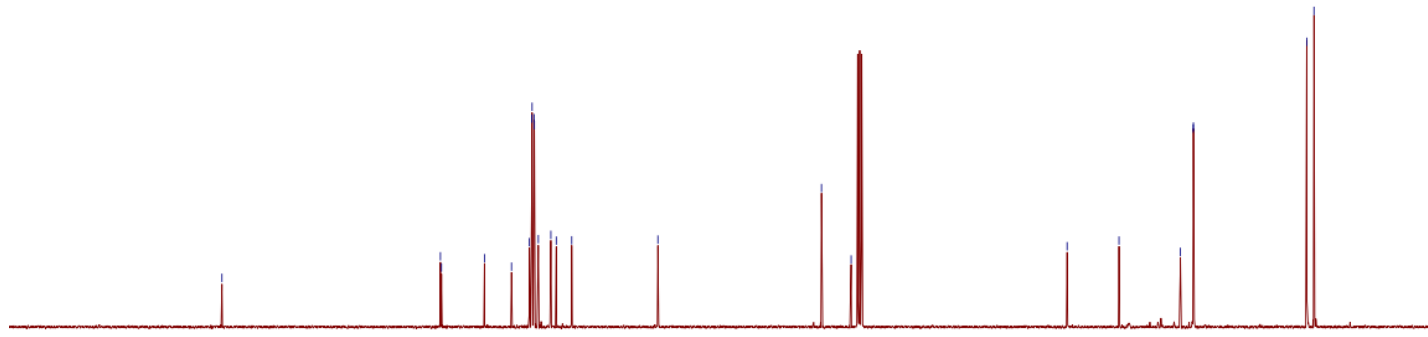

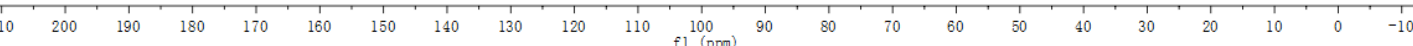


${ }^{11} \mathrm{~B}$ NMR Spectrum of Compound 6c (193 $\left.\mathrm{MHz}, \mathrm{CDCl}_{3}\right)$

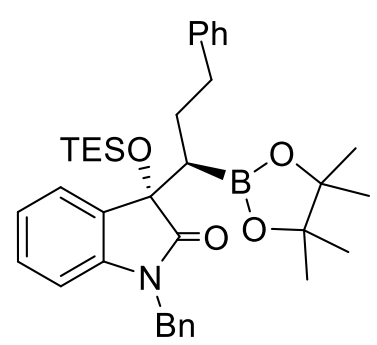

6c

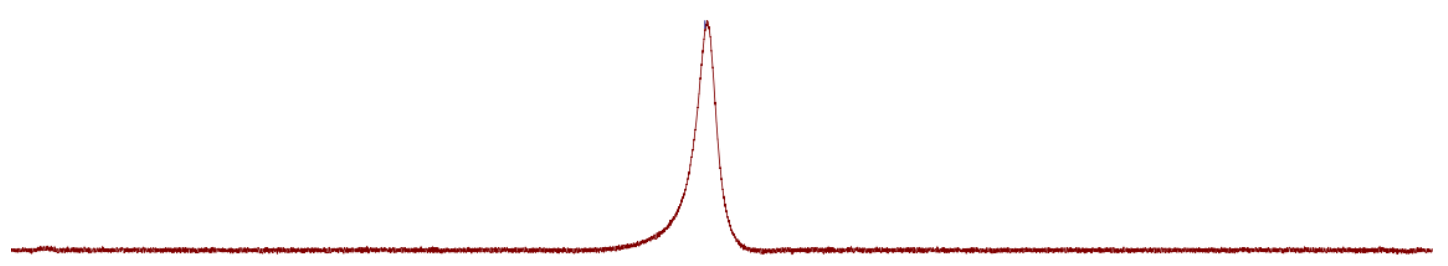


${ }^{1} \mathrm{H}$ NMR Spectrum of Compound $6 \mathrm{~d}\left(400 \mathrm{MHz}, \mathrm{CDCl}_{3}\right)$

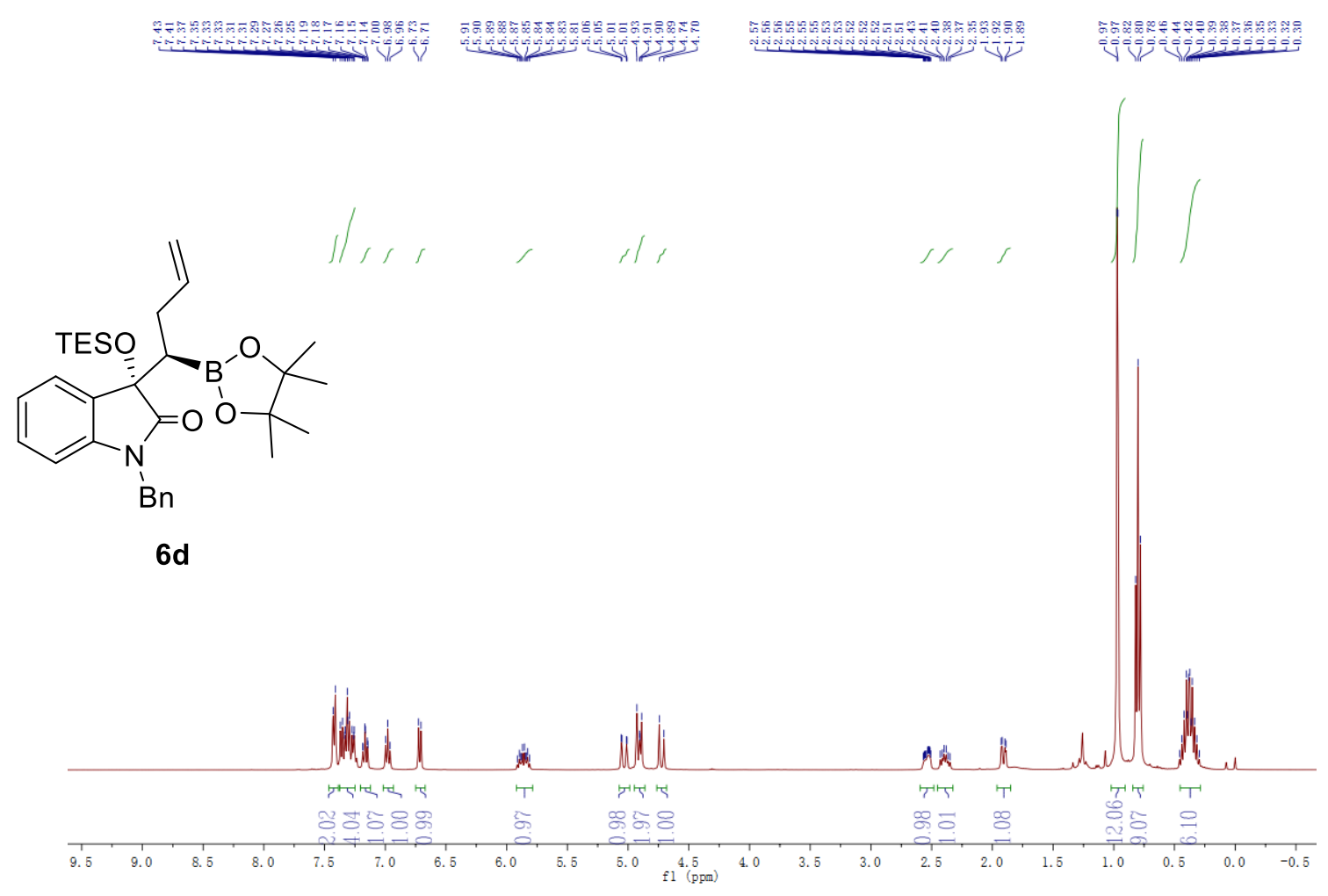

${ }^{13} \mathrm{C}$ NMR Spectrum of Compound $6 \mathrm{~d}\left(101 \mathrm{MHz}, \mathrm{CDCl}_{3}\right)$

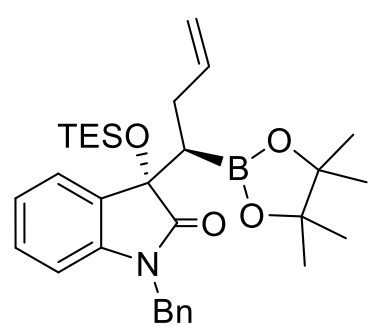

6d

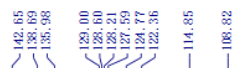

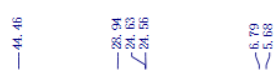

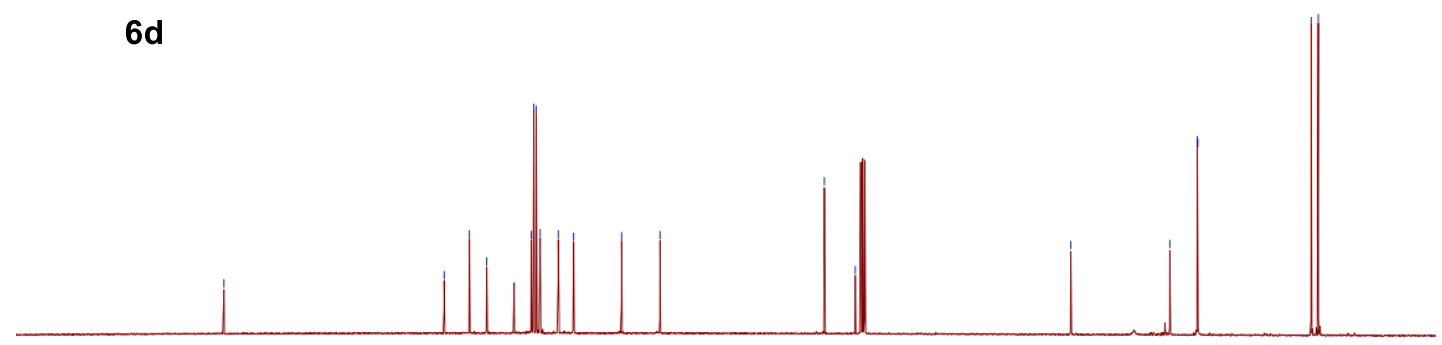

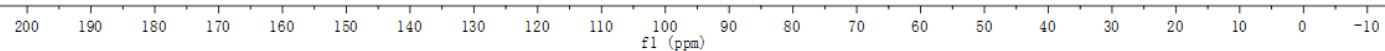


${ }^{11} \mathrm{~B}$ NMR Spectrum of Compound $6 \mathrm{~d}\left(193 \mathrm{MHz}, \mathrm{CDCl}_{3}\right)$

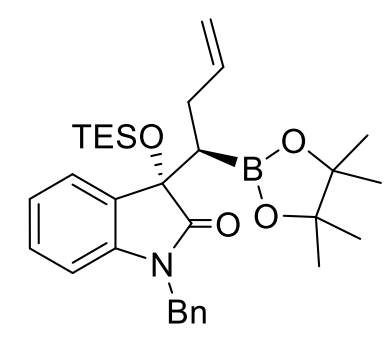

$6 d$

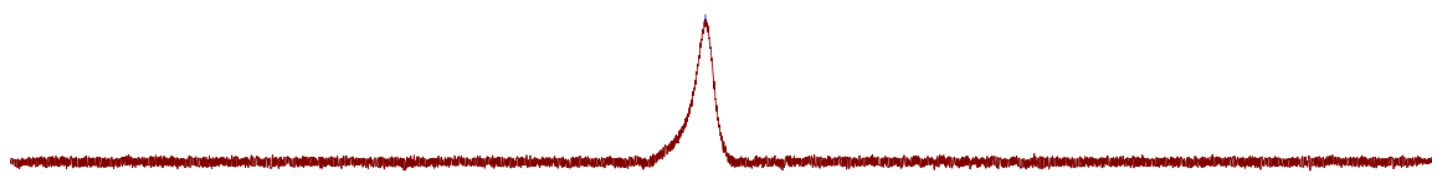


${ }^{1} \mathrm{H}$ NMR Spectrum of Compound $6 \mathrm{e}\left(400 \mathrm{MHz}, \mathrm{CDCl}_{3}\right)$

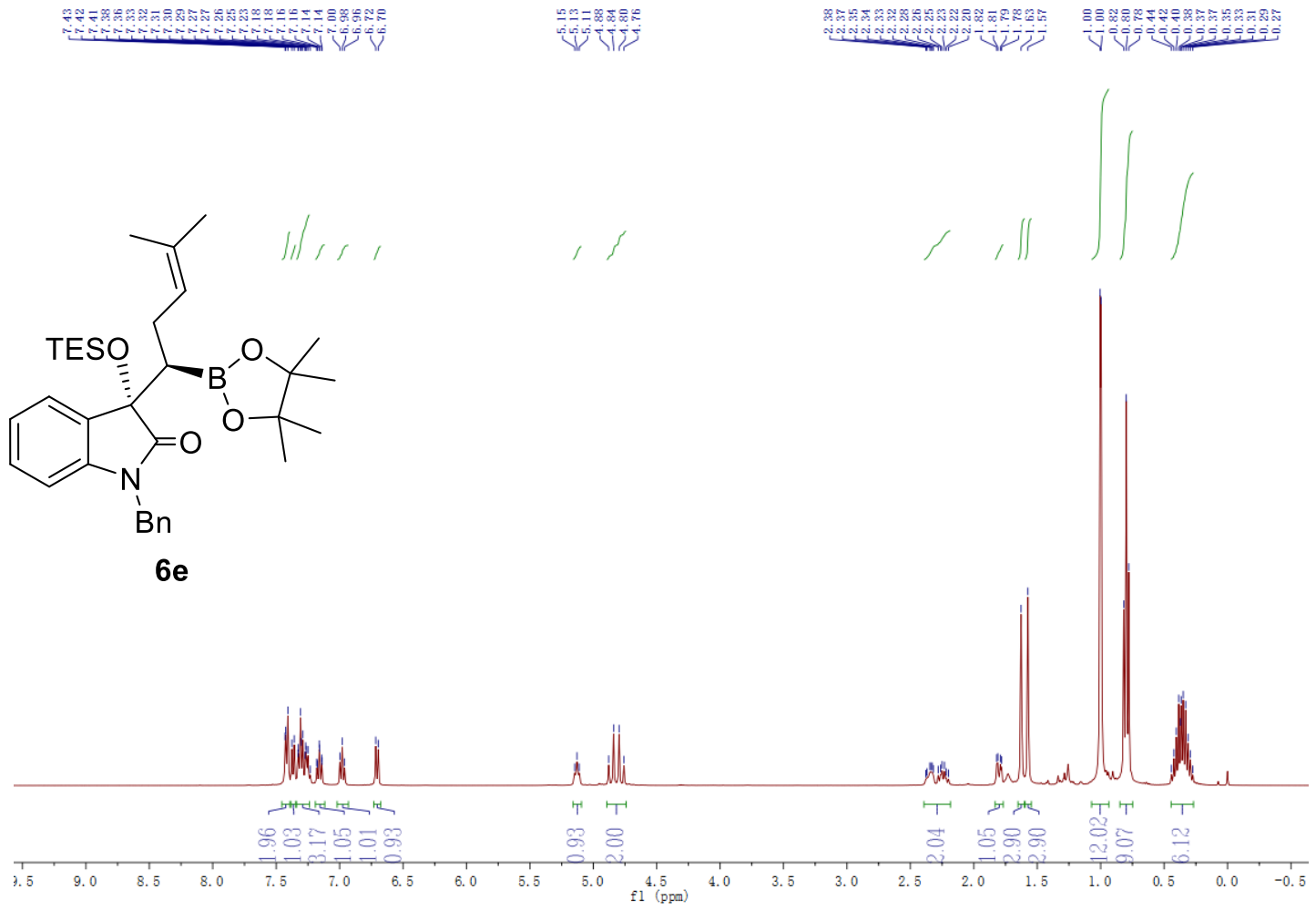

${ }^{13} \mathrm{C}$ NMR Spectrum of Compound $6 \mathrm{e}\left(101 \mathrm{MHz}, \mathrm{CDCl}_{3}\right)$

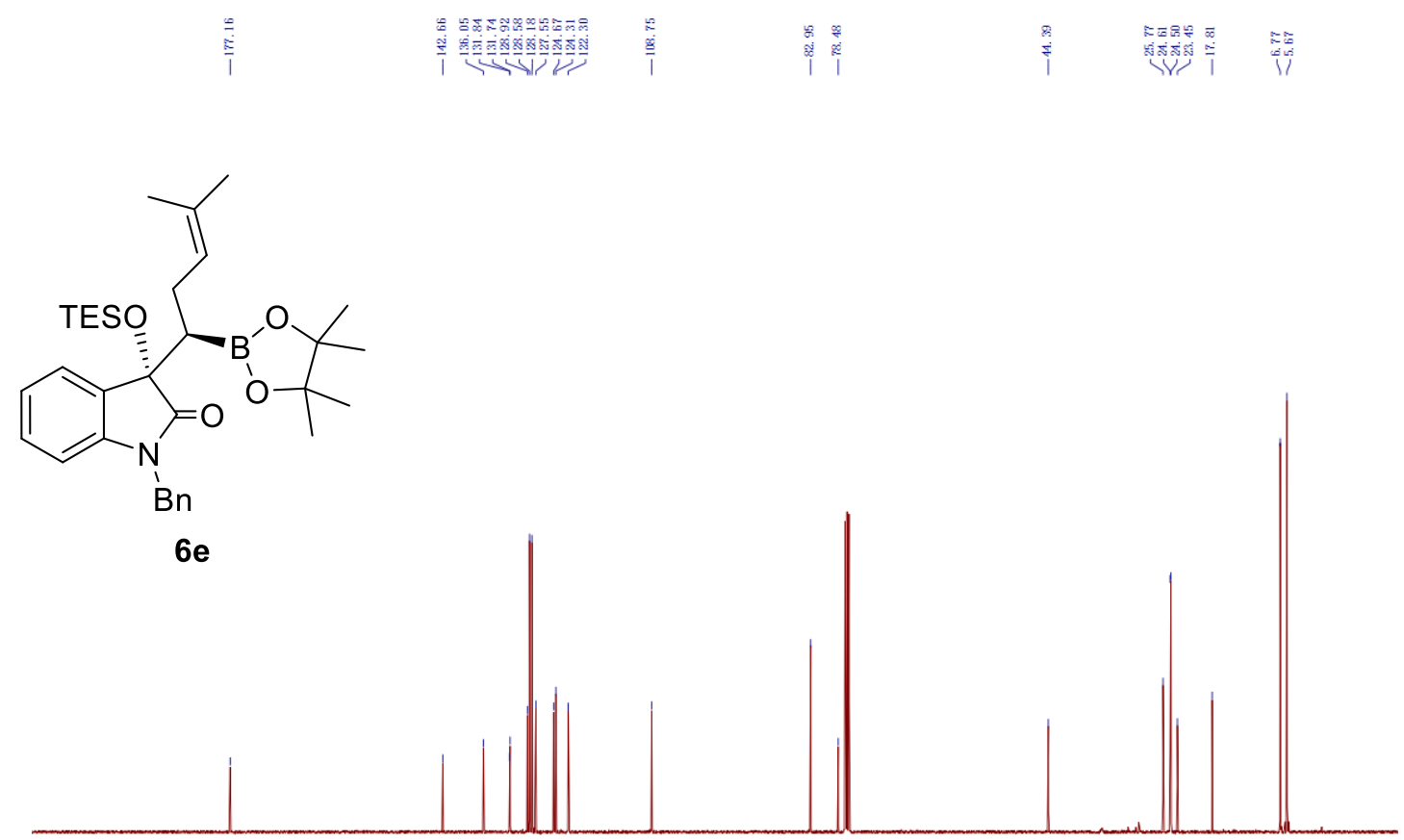

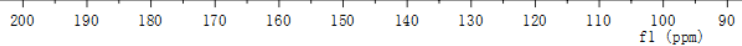


${ }^{11} \mathrm{~B}$ NMR Spectrum of Compound $6 \mathrm{e}\left(193 \mathrm{MHz}, \mathrm{CDCl}_{3}\right)$

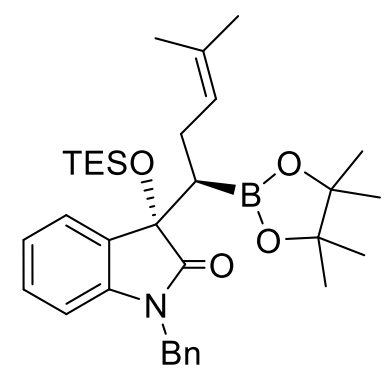

6 e

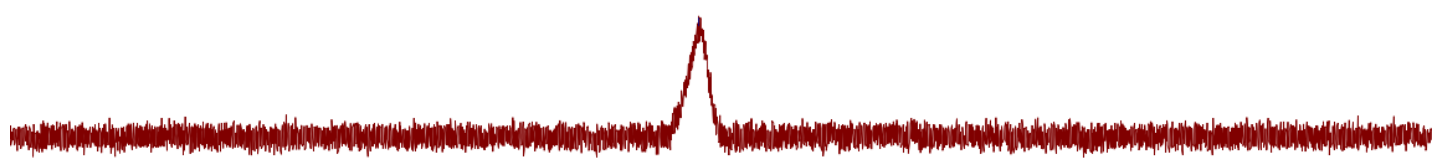


${ }^{1} \mathrm{H}$ NMR Spectrum of Compound $6 \mathrm{f}\left(400 \mathrm{MHz}, \mathrm{CDCl}_{3}\right)$

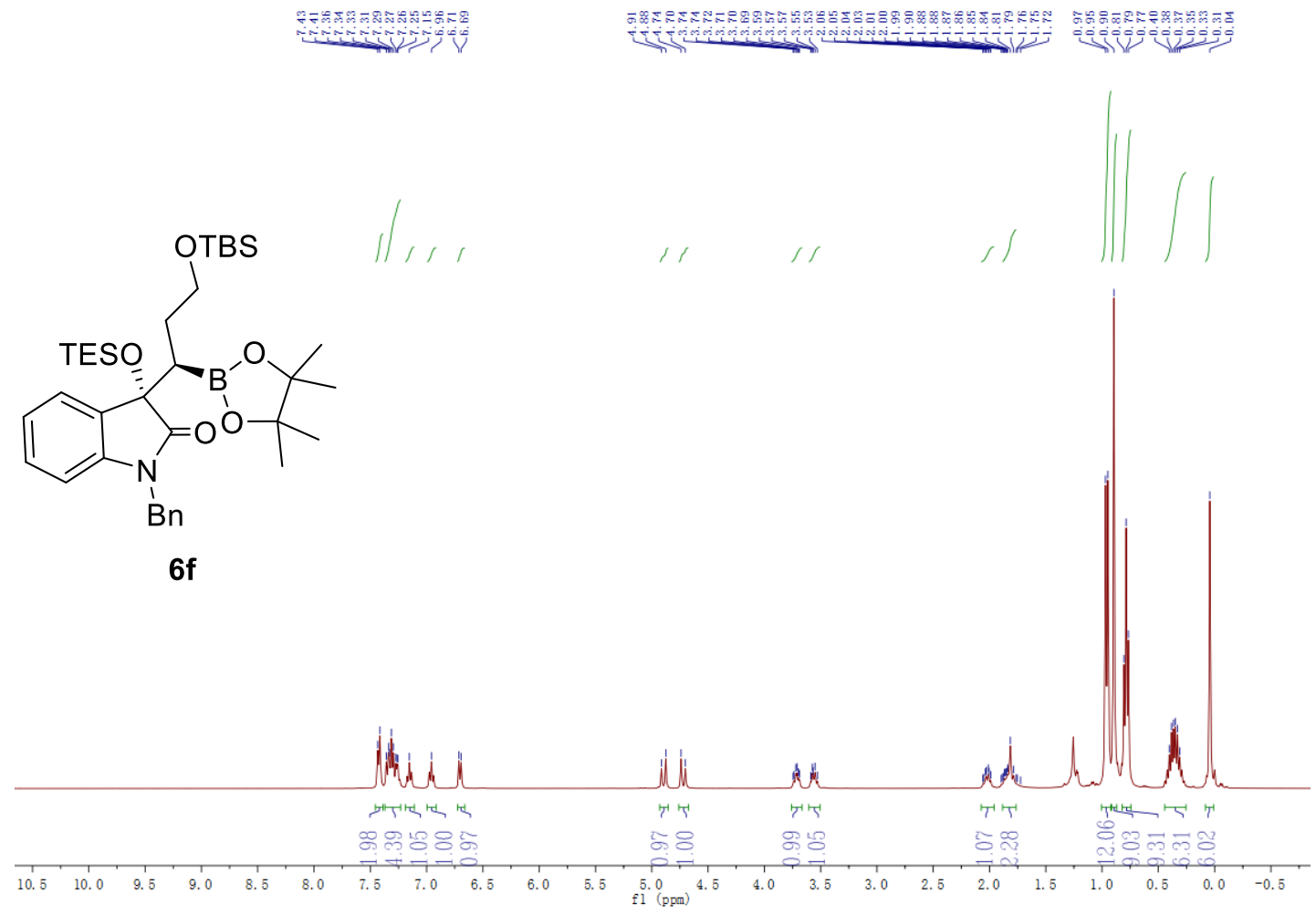

${ }^{13} \mathrm{C}$ NMR Spectrum of Compound $6 \mathrm{f}\left(101 \mathrm{MHz}, \mathrm{CDCl}_{3}\right)$

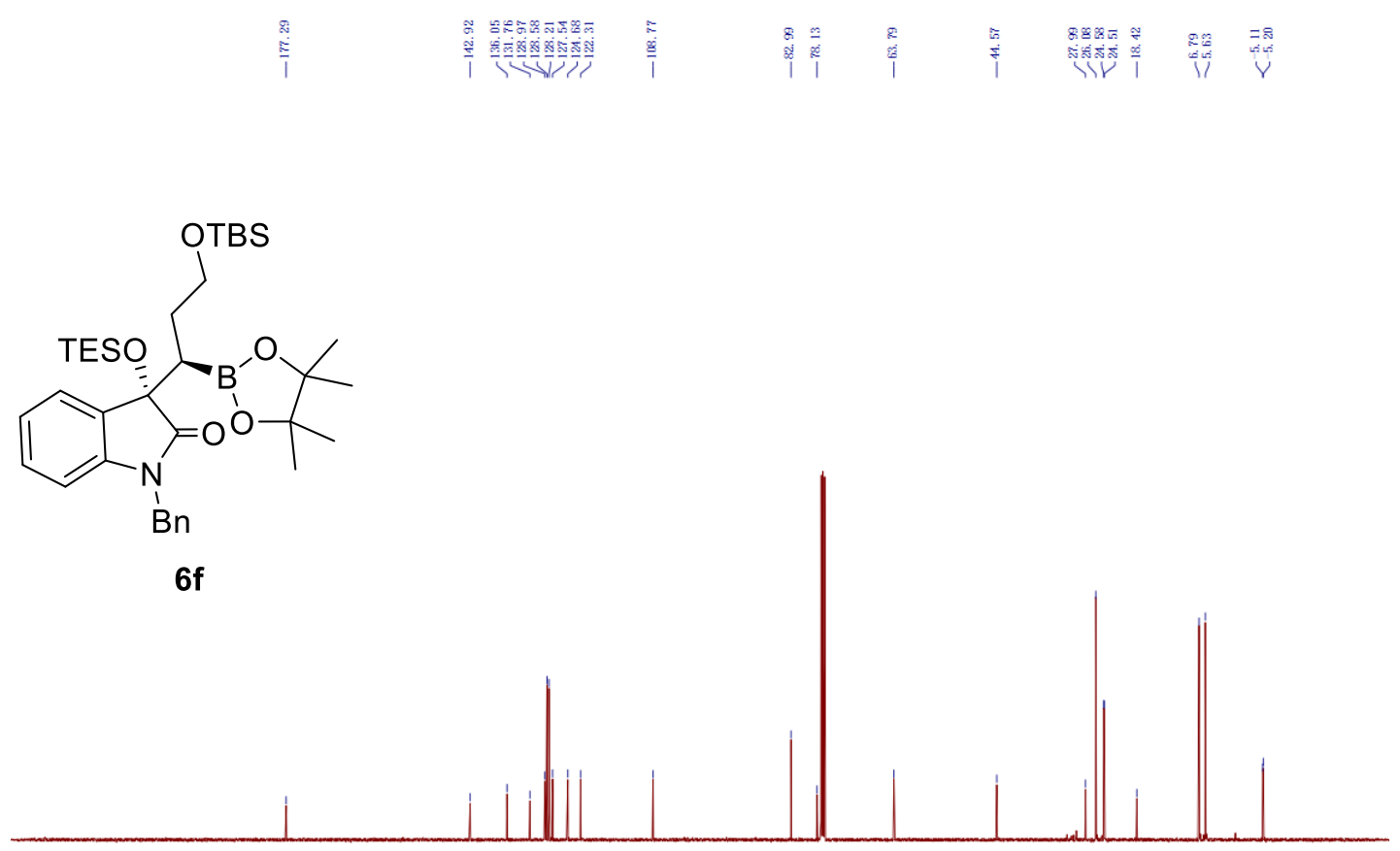

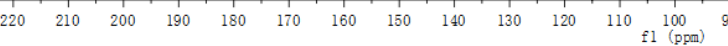


${ }^{11} \mathrm{~B}$ NMR Spectrum of Compound $6 f\left(193 \mathrm{MHz}, \mathrm{CDCl}_{3}\right)$

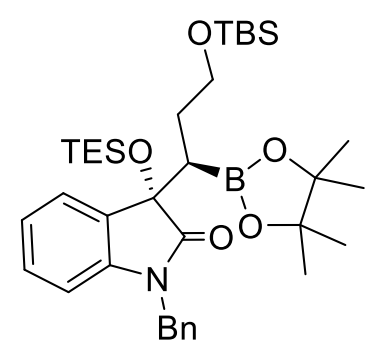

$6 f$

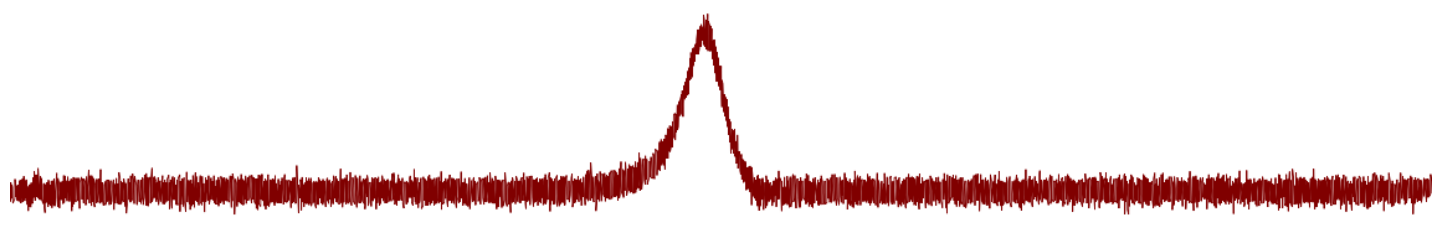


${ }^{1} \mathrm{H}$ NMR Spectrum of Compound $7\left(400 \mathrm{MHz}, \mathrm{CDCl}_{3}\right)$

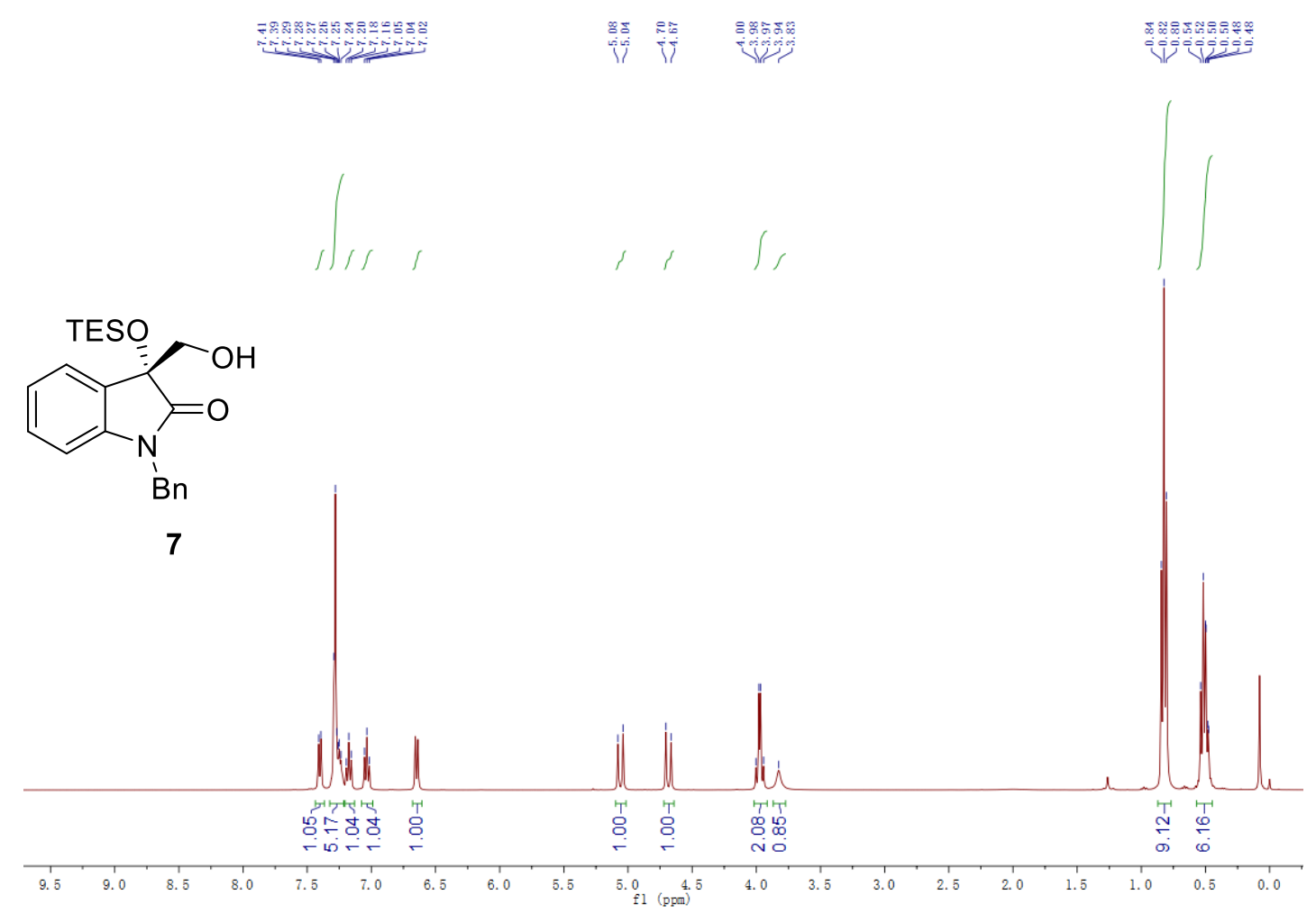

${ }^{13} \mathrm{C}$ NMR Spectrum of Compound $7\left(101 \mathrm{MHz}, \mathrm{CDCl}_{3}\right)$
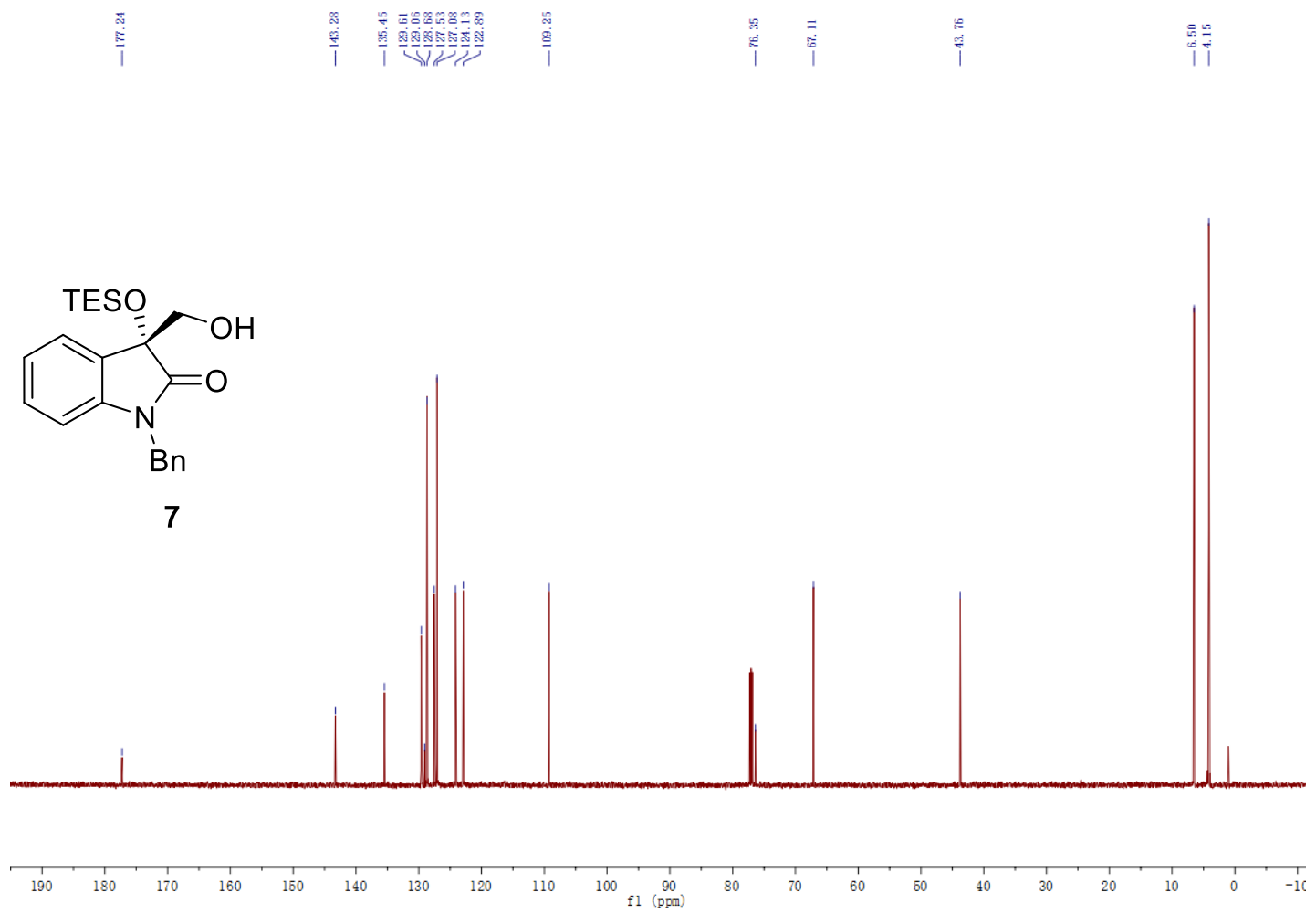
${ }^{1} \mathrm{H}$ NMR Spectrum of Compound 8 (400 MHz, DMSO-d6)

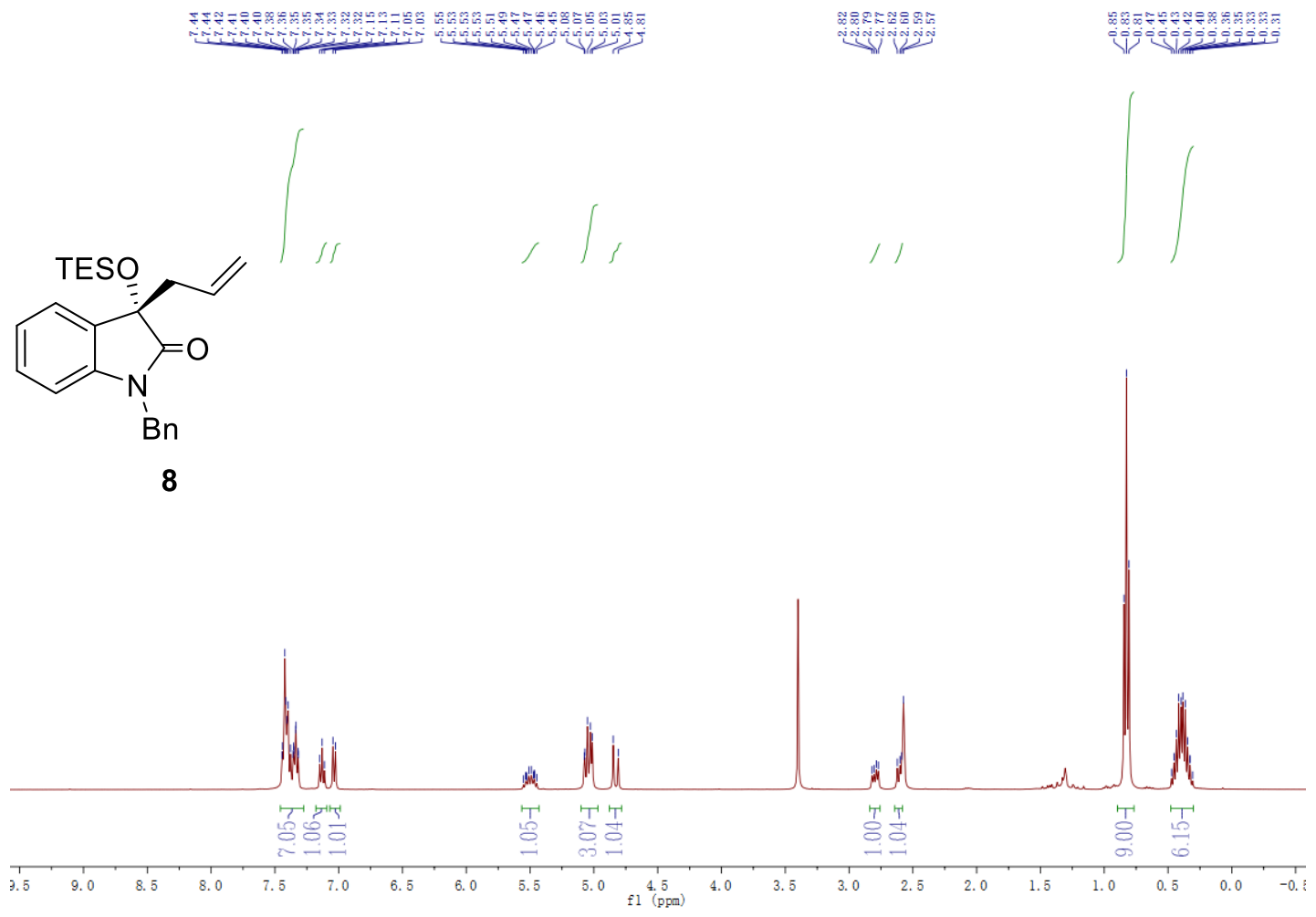

${ }^{13} \mathrm{C}$ NMR Spectrum of Compound 8 (101 MHz, DMSO-d6)

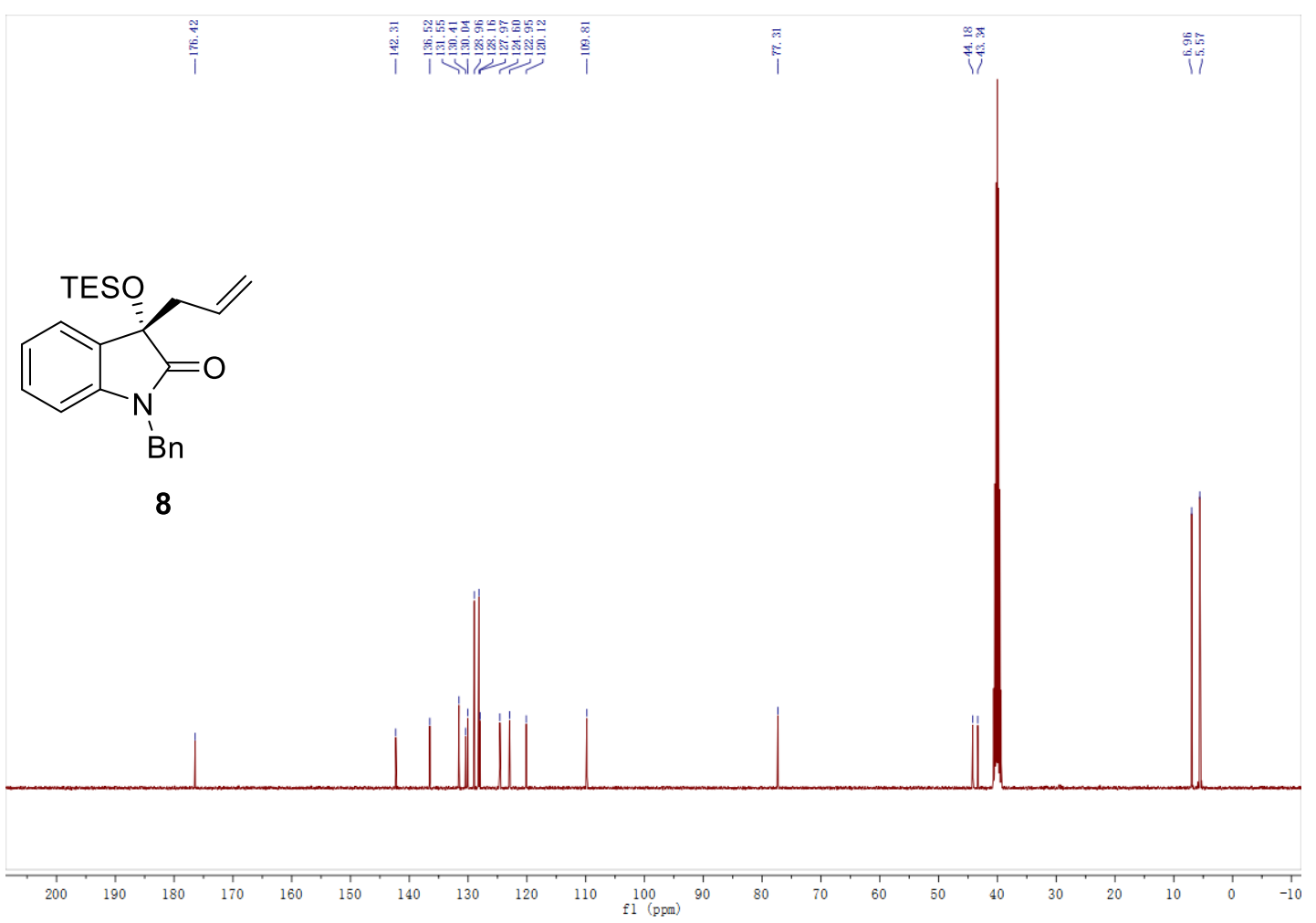


${ }^{1} \mathrm{H}$ NMR Spectrum of Compound $9\left(400 \mathrm{MHz}, \mathrm{CDCl}_{3}\right)$

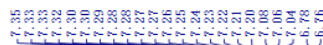

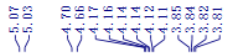

Nivind

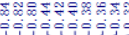

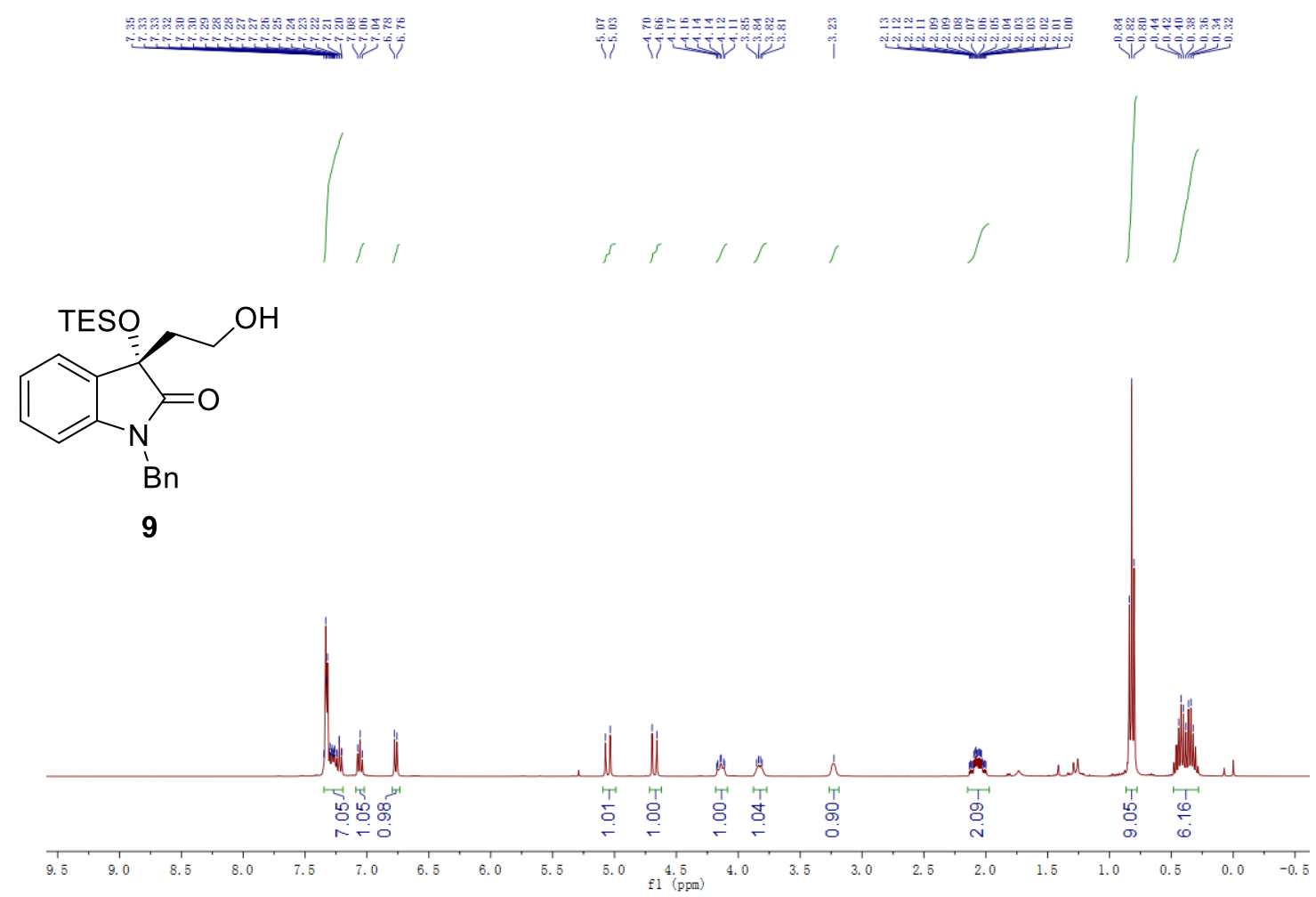

${ }^{13} \mathrm{C}$ NMR Spectrum of Compound $9\left(101 \mathrm{MHz}, \mathrm{CDCl}_{3}\right)$
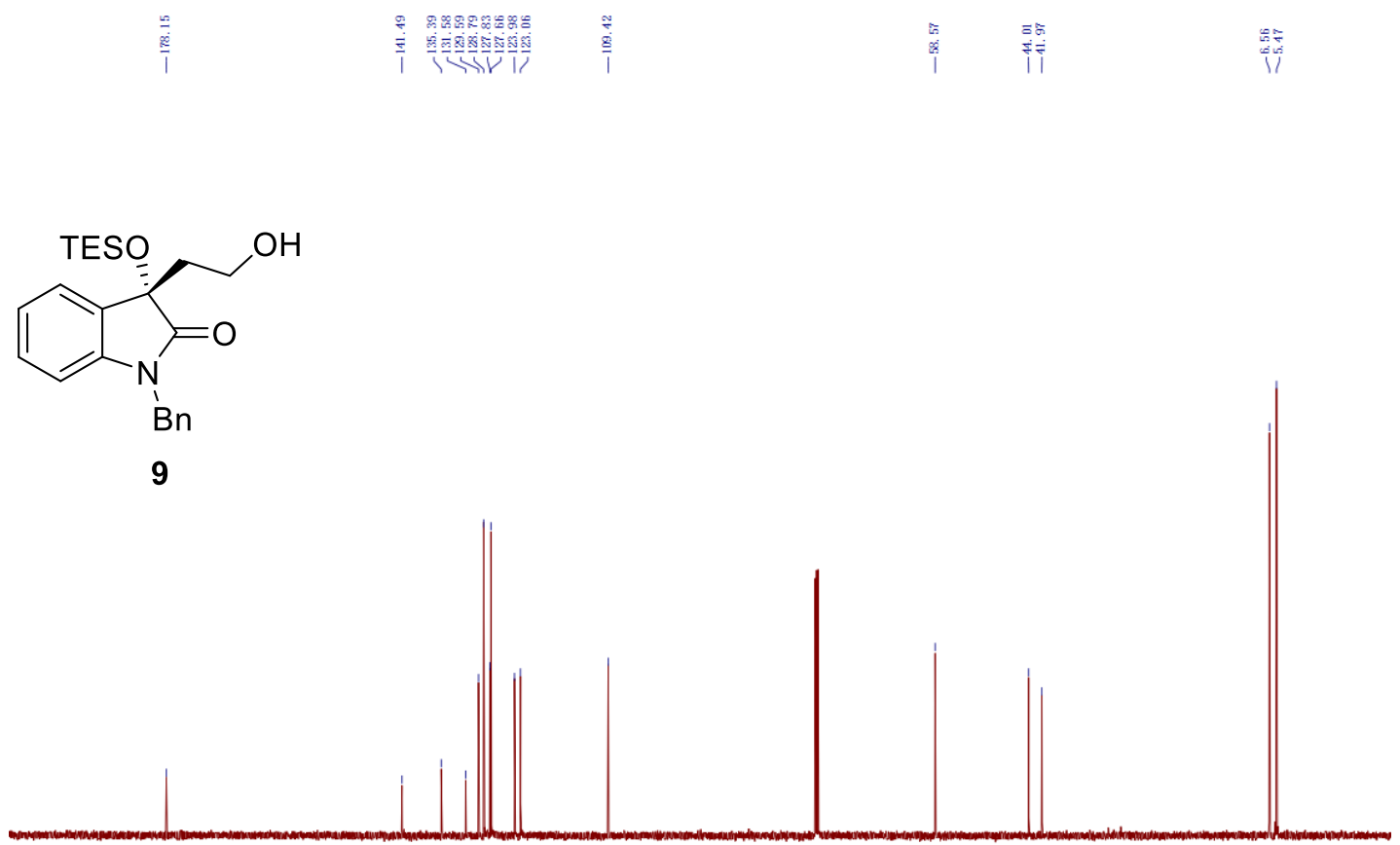

$\begin{array}{lllllllllll}200 & 190 & 180 & 170 & 160 & 150 & 140 & 130 & 120 & 110 & 100 \\ \mathrm{f} 1 & 900\end{array}$ 
${ }^{1} \mathrm{H}$ NMR Spectrum of Compound 10 (400 MHz, DMSO-d6)

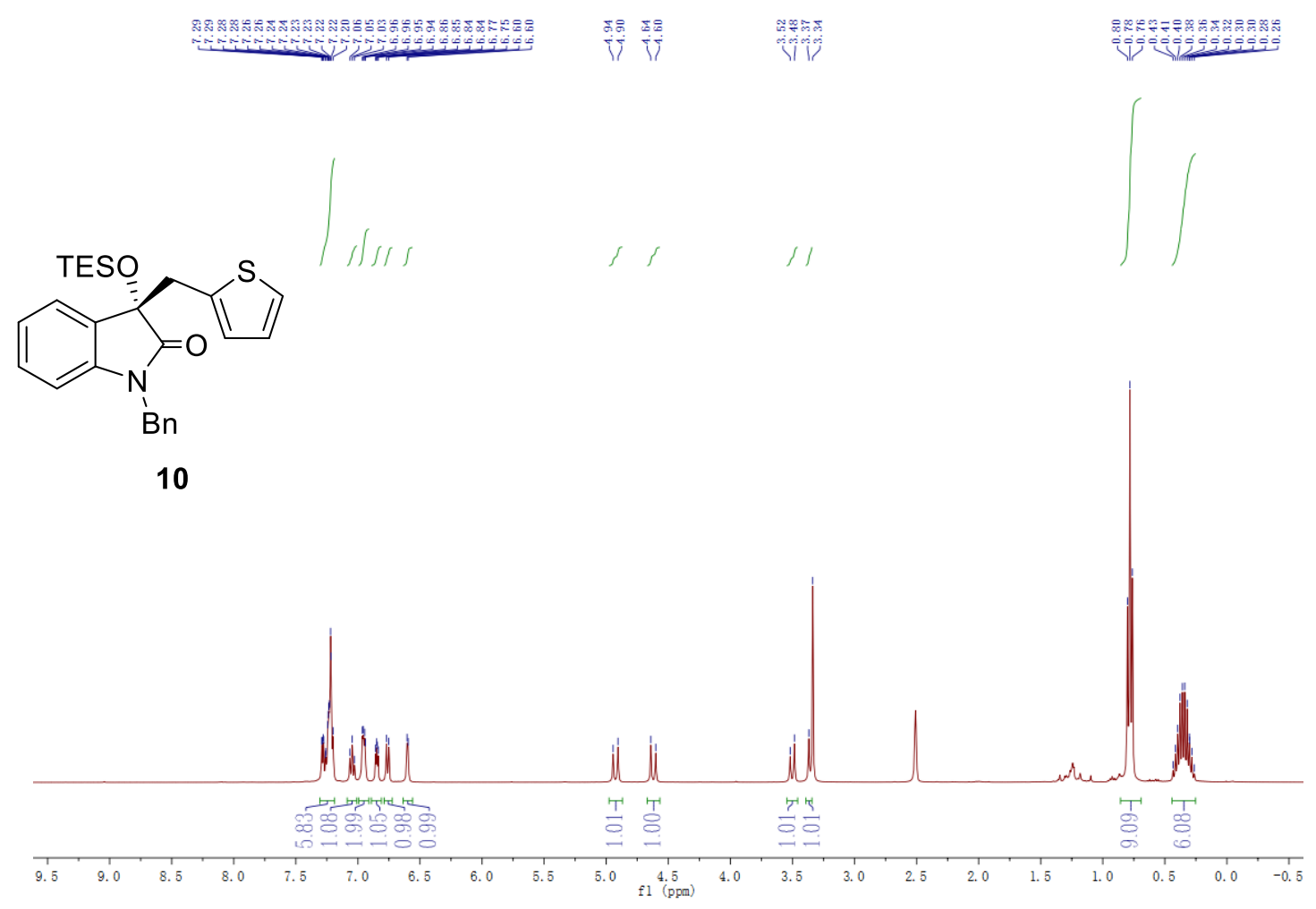

${ }^{13} \mathrm{C}$ NMR Spectrum of Compound 10 (101 MHz, DMSO-d6)

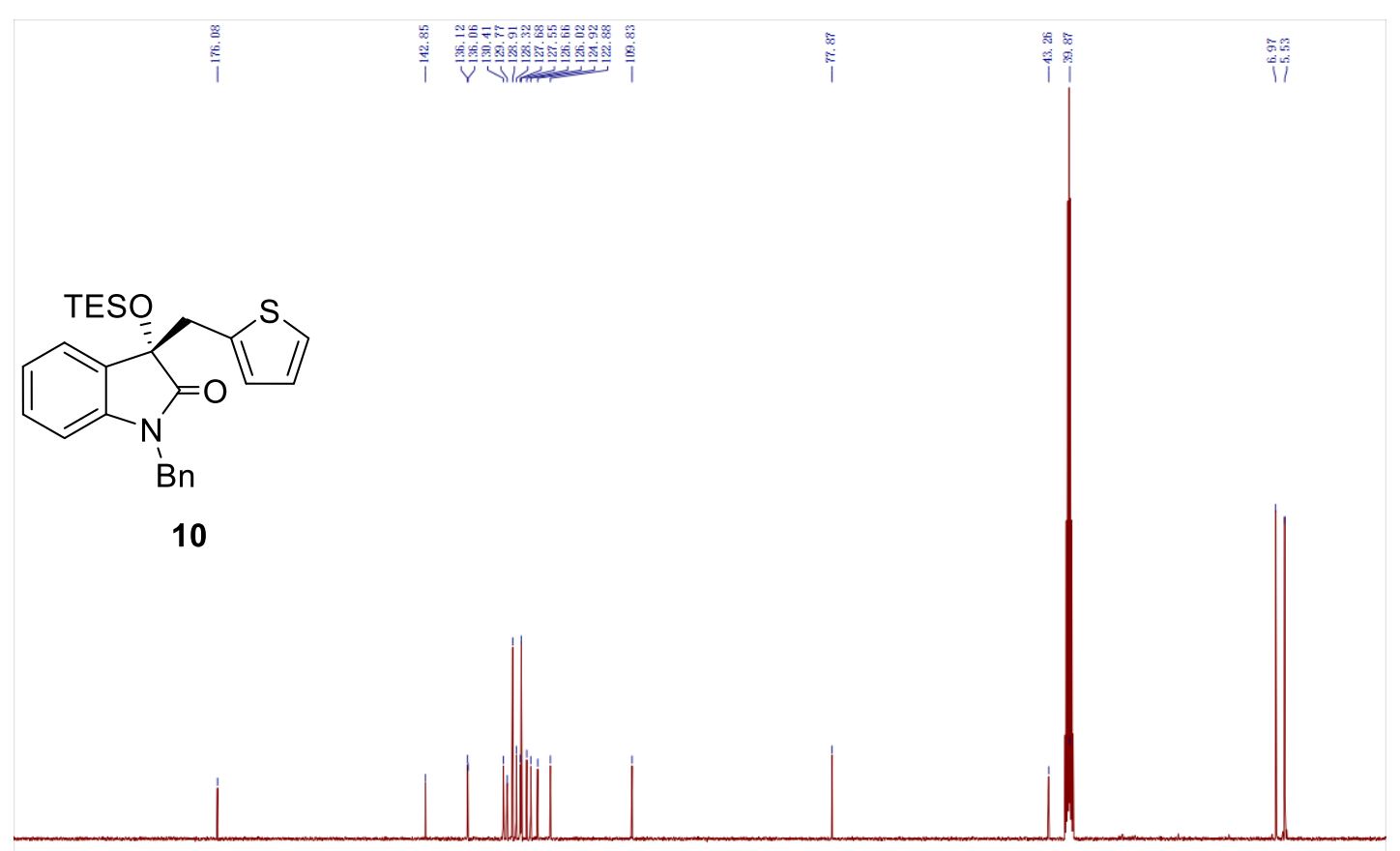

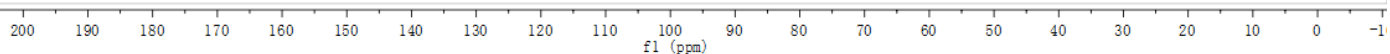


${ }^{1} \mathrm{H}$ NMR Spectrum of Compound 11 (400 MHz, DMSO-d6)

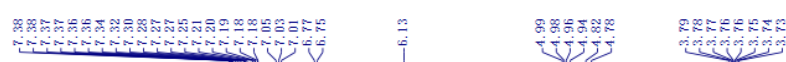
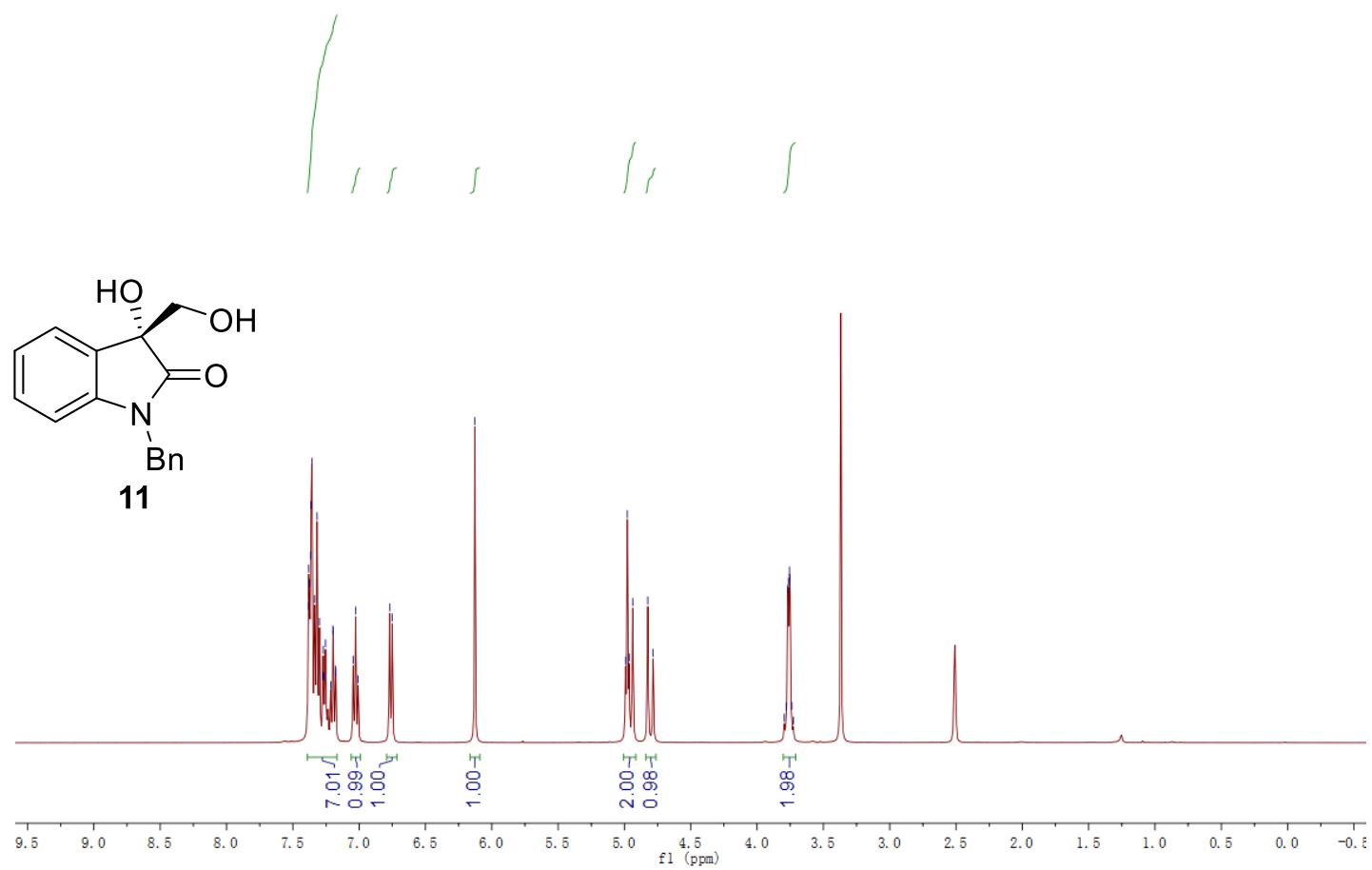

${ }^{13} \mathrm{C}$ NMR Spectrum of Compound 11 (101 MHz, DMSO-d6)

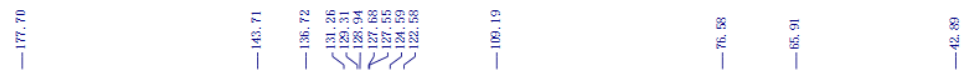<smiles>O=C1N(Cc2ccccc2)c2ccccc2[C@]1(O)CO</smiles>

11

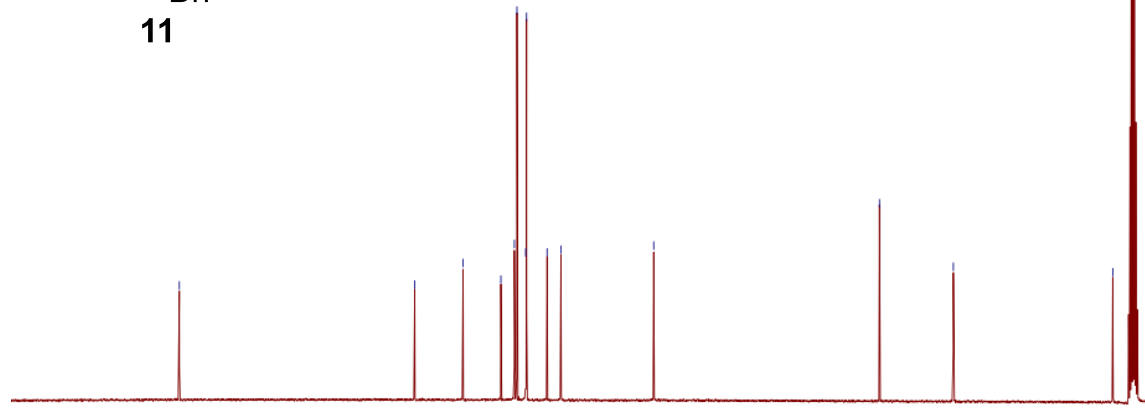

To0

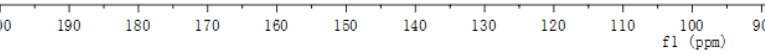


${ }^{1} \mathrm{H}$ NMR Spectrum of Compound 12 (400 MHz, DMSO-d6)

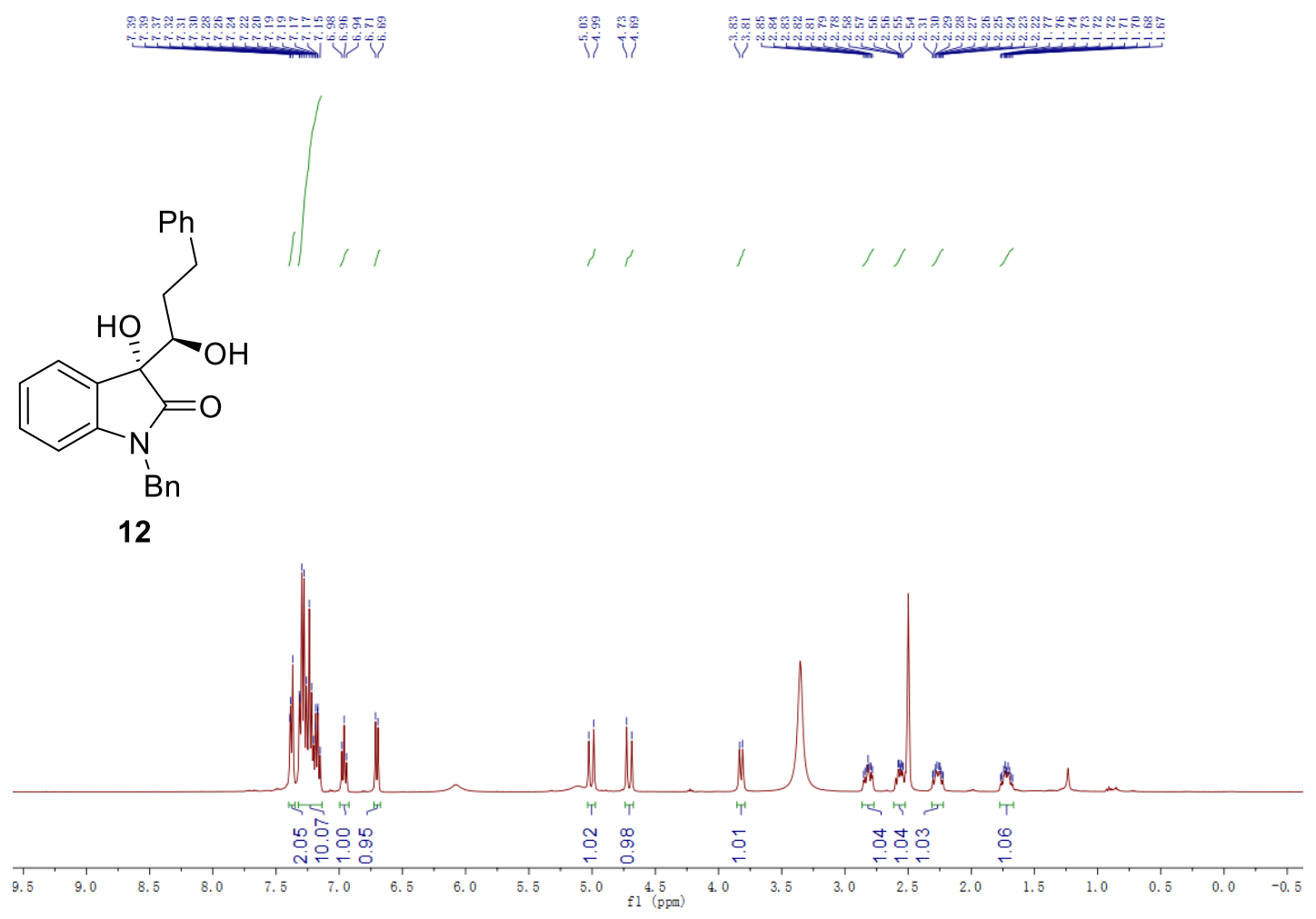

${ }^{13} \mathrm{C}$ NMR Spectrum of Compound 12 (101 MHz, DMSO-d6)

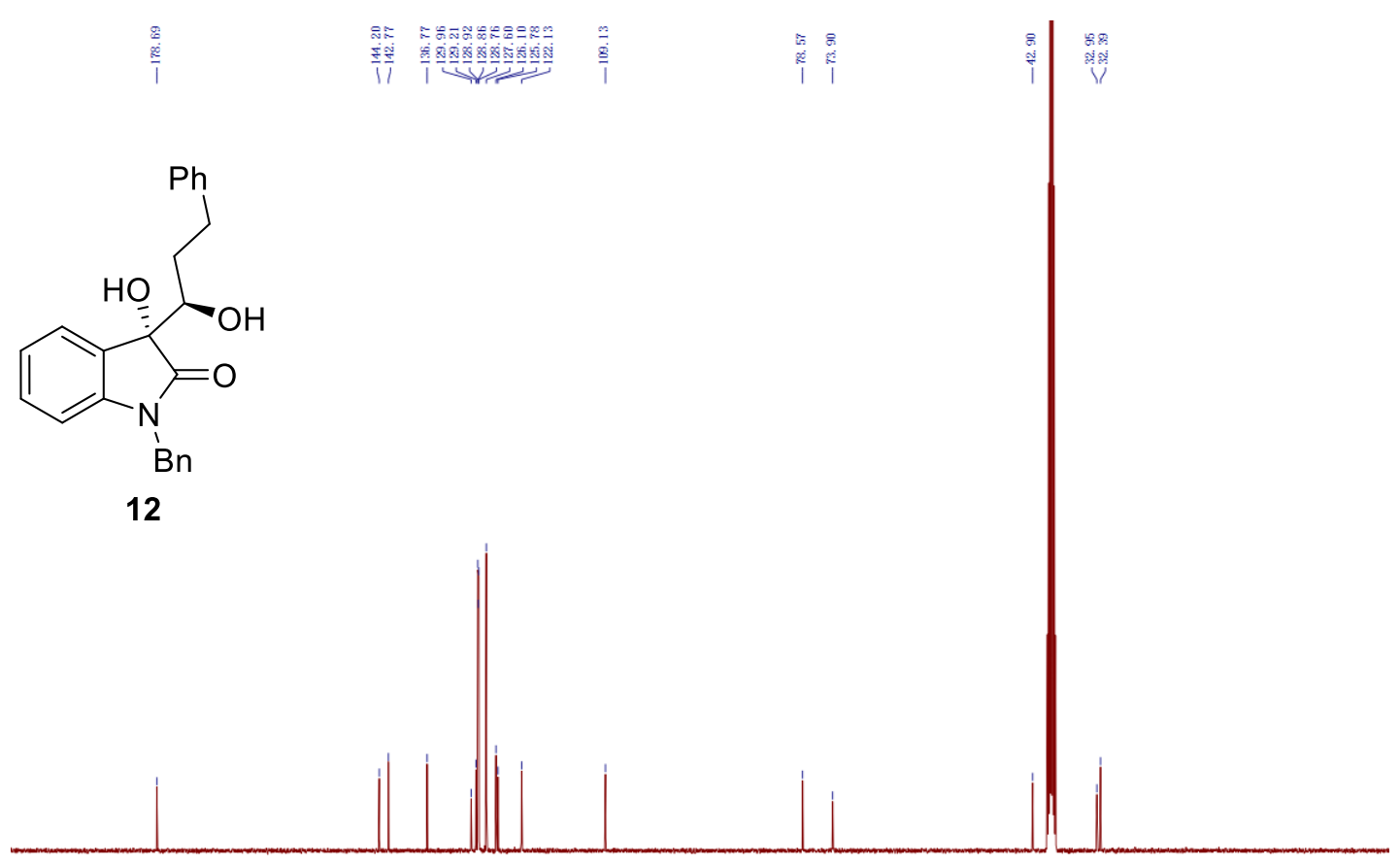

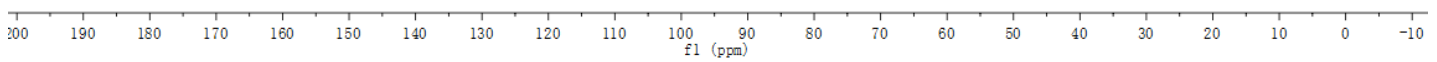




\section{HPLC Spectra}

DAD1B, Sig=254, 4 Ref=off

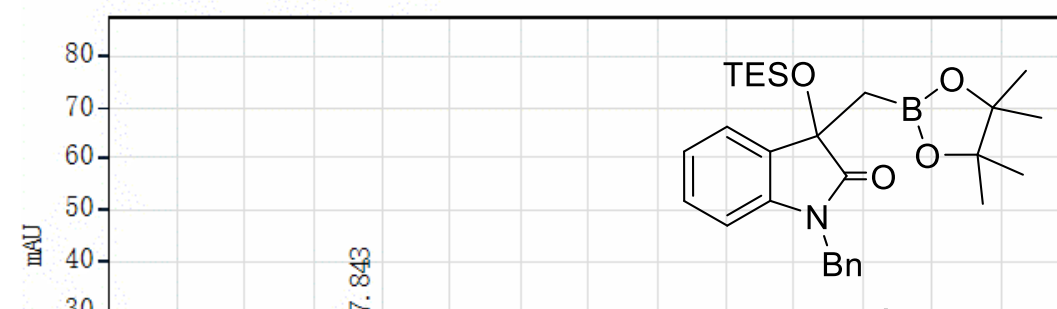

rac-4a

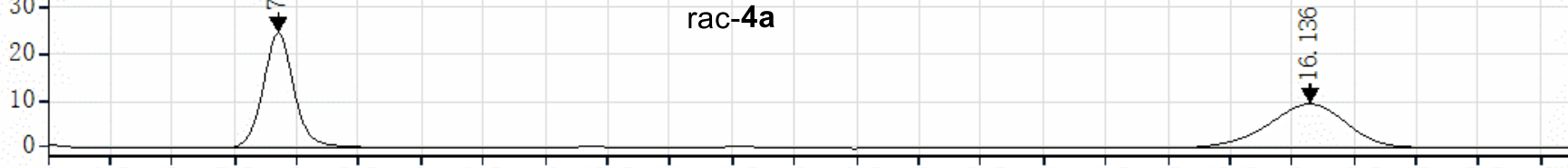

\begin{tabular}{ccccc}
\hline RetTime [min] & Type & Wide [min] & Area[mAu*s & Height[mAu] \\
\hline 7.843 & MM m & 0.27 & 432.05 & 24.81 \\
16.136 & MM m & 0.56 & 425.41 & 9.51
\end{tabular}

DAD1B, Sig=254, 4 Ref $=$ off

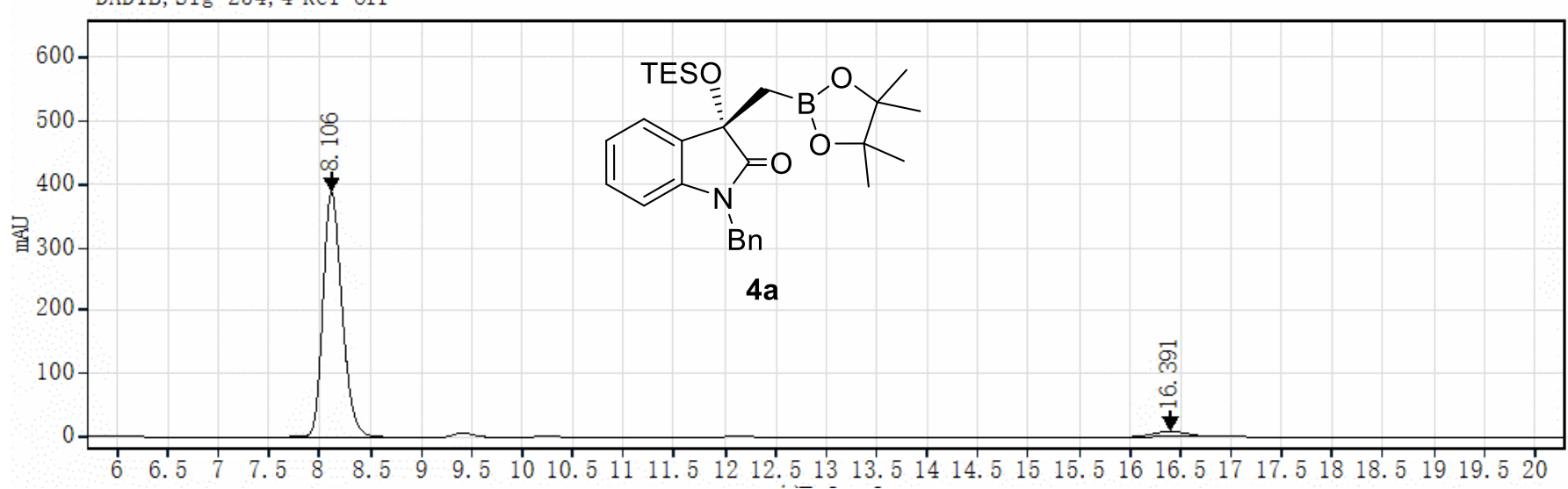

\begin{tabular}{ccccccccc}
\hline RetTime [min] & Type & Wide [min] & Area[mAu*s] & Height[mAu] & 5118.62 & 388.39 \\
16.391 & MM m & 0.20 & 185.57 & 7.98
\end{tabular}


DAD1B, Sig=254, 4 Ref=off

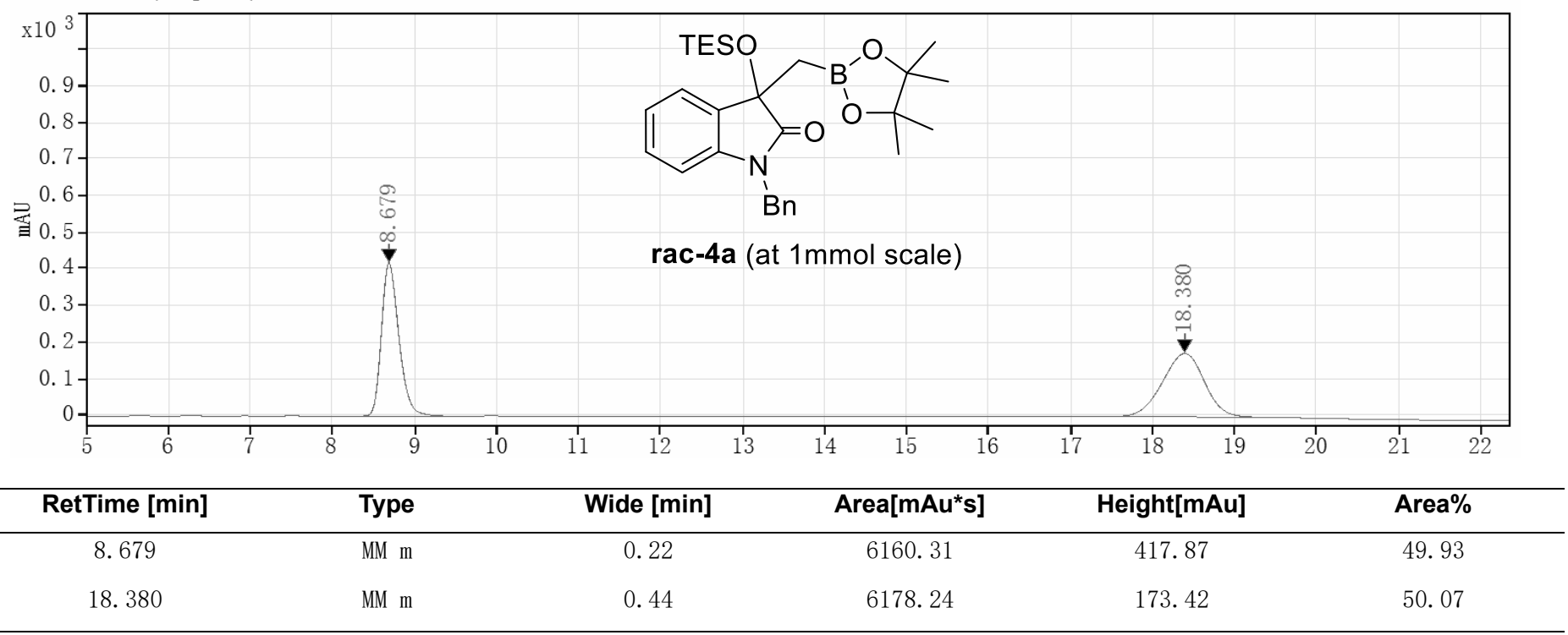

DAD1B, Sig=254, 4 Ref=off

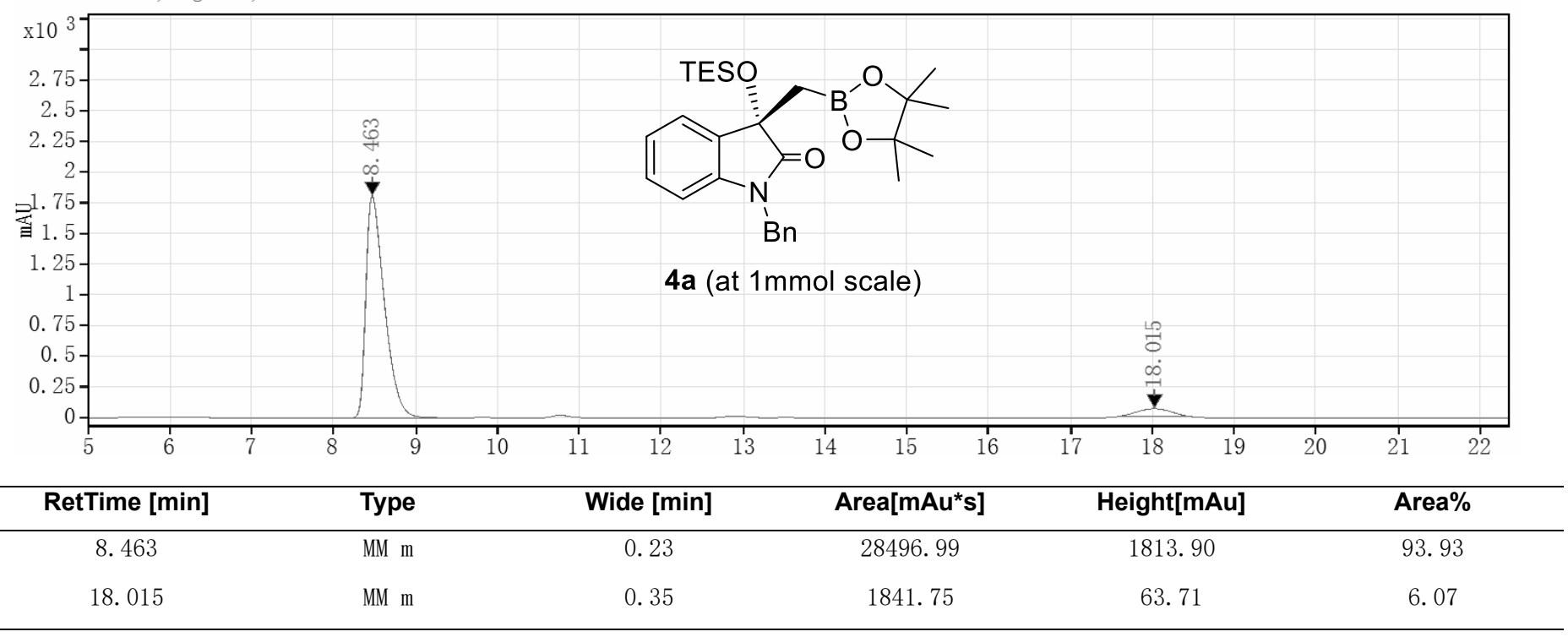


DAD1B, Sig=254, 4 Ref=off

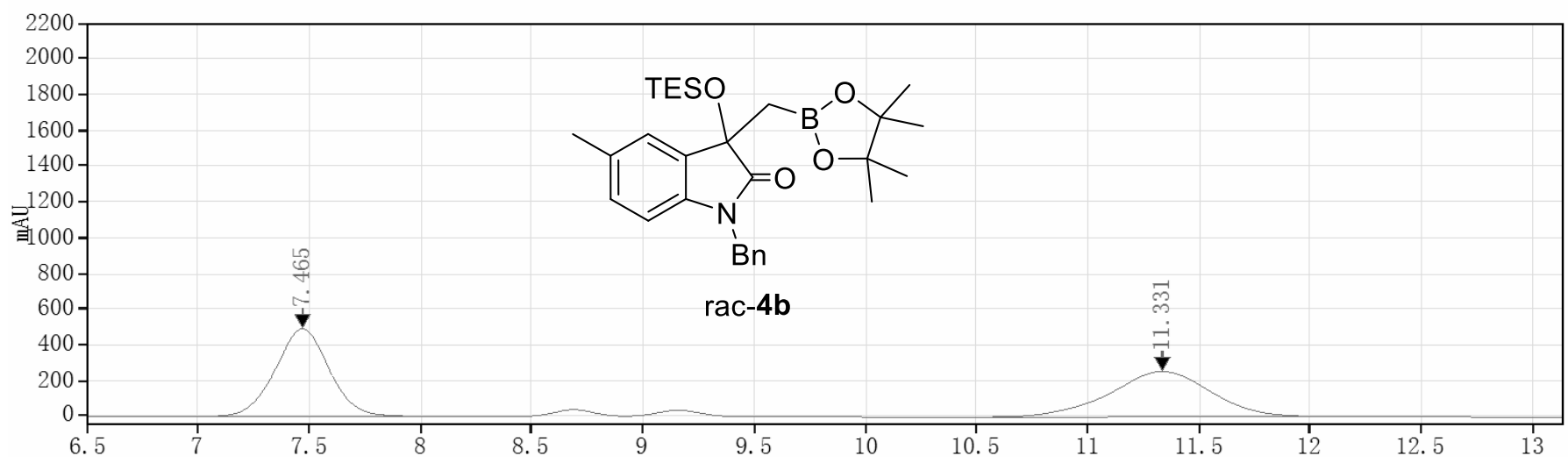

\begin{tabular}{ccccc}
\hline RetTime [min] & Type & Wide [min] & Area[mAu*s] & Height[mAu] \\
\hline 7.465 & MM m & 0.26 & 8379.85 & 492.61 \\
11.331 & MM m & 0.48 & 8107.68 & 254.50 \\
\hline
\end{tabular}

DAD1B, Sig=254, $4 \quad$ Ref=off

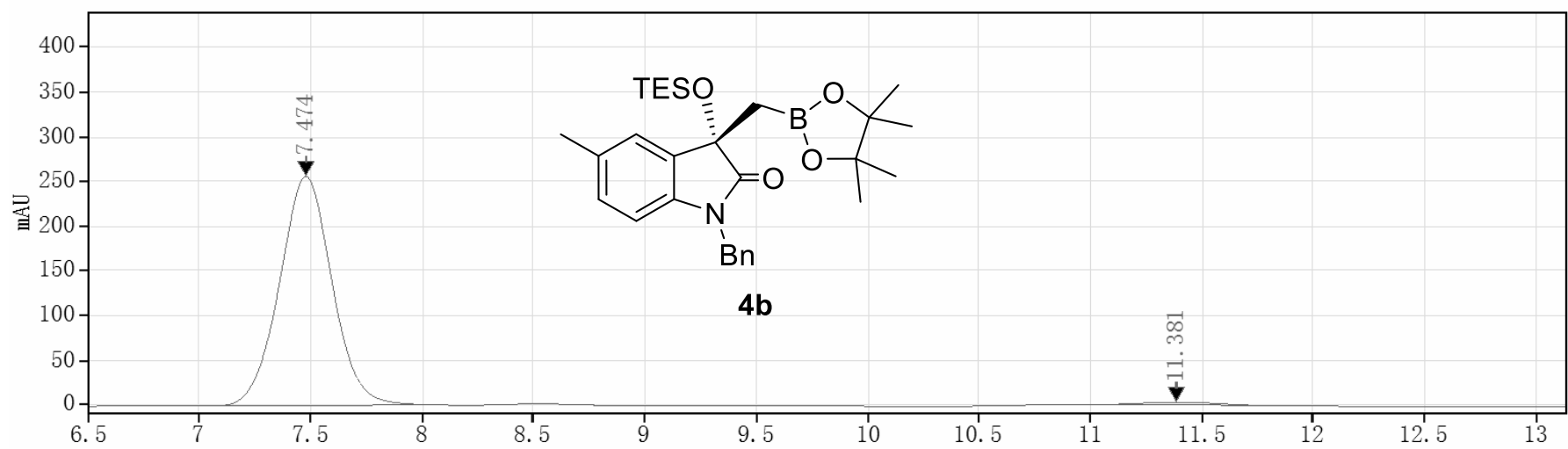

\begin{tabular}{ccccc}
\hline RetTime [min] & Type & Wide [min] & Area[mAu*s] & Height[mAu] \\
\hline 7.474 & MM m & 0.25 & 4196.49 & 256.09 \\
11.381 & MM m & 0.28 & 68.87 & 3.09 \\
\hline
\end{tabular}


DAD1B, Sig=254, 4 Ref=off

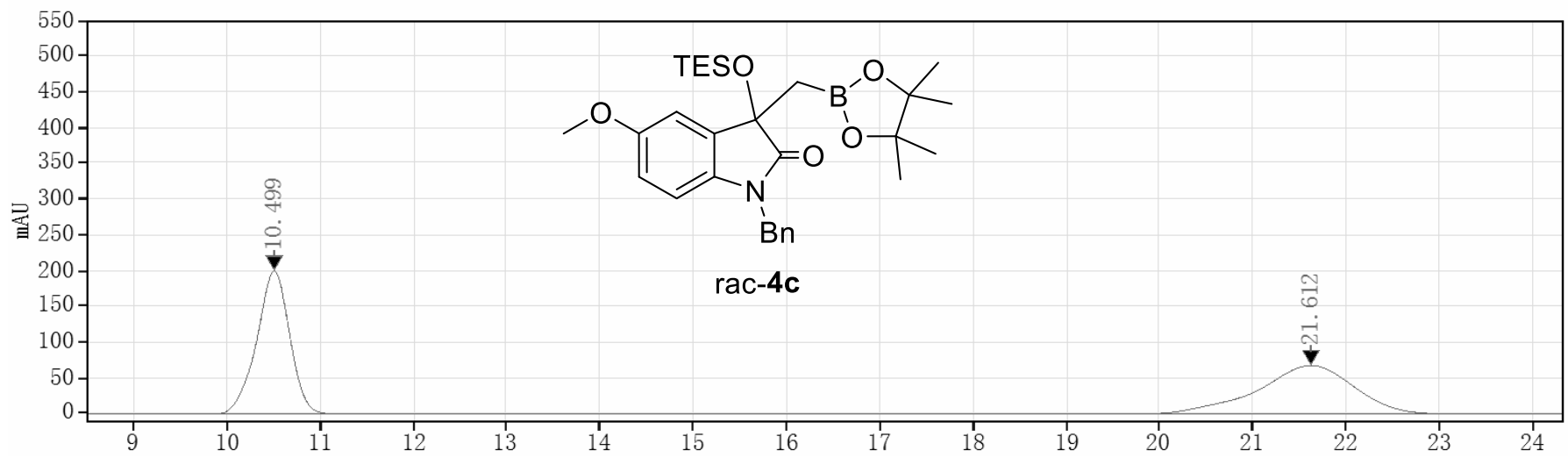

\begin{tabular}{ccccc}
\hline RetTime [min] & Type & Wide [min] & Area[mAu*s] & Height[mAu] \\
\hline 10.499 & MM m & 0.37 & 4938.41 & 200.36 \\
21.612 & MM m & 1.03 & 4946.96 & 68.18 \\
\hline
\end{tabular}

DAD1B, Sig=254, 4 Ref=off

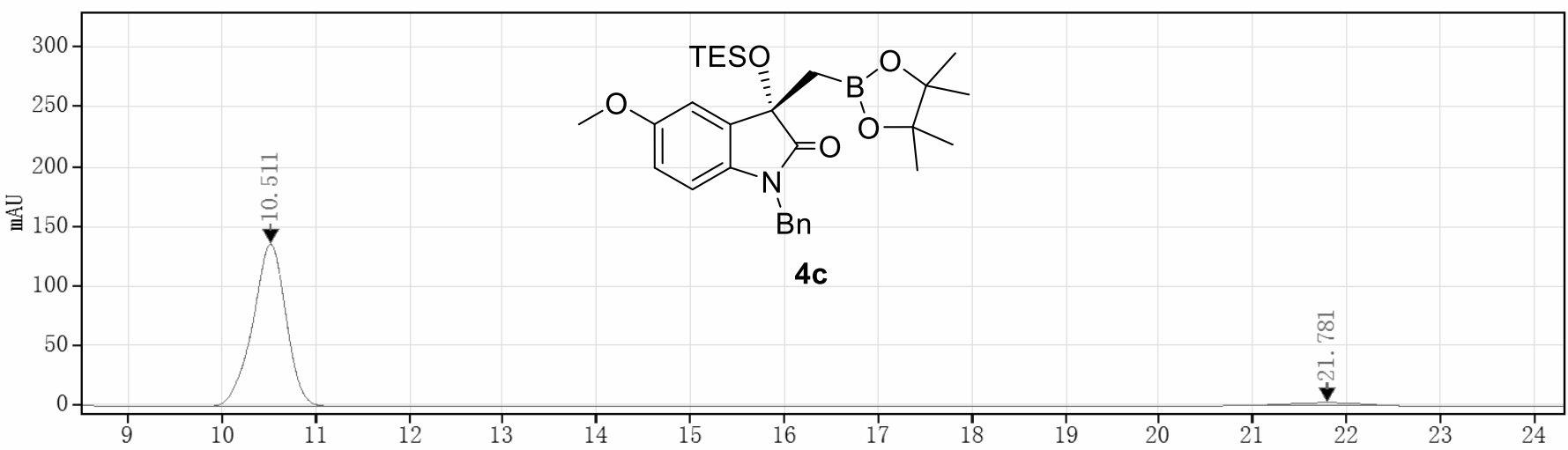

\begin{tabular}{ccccc}
\hline RetTime [min] & Type & Wide [min] & Area[mAu*s] & Height[mAu] \\
\hline 10.511 & MM m & 0.37 & 3361.60 & 135.94 \\
21.781 & MM m & 0.61 & 124.31 & 2.39 \\
\hline
\end{tabular}


DAD1B, Sig=254, $4 \quad$ Ref $=$ off

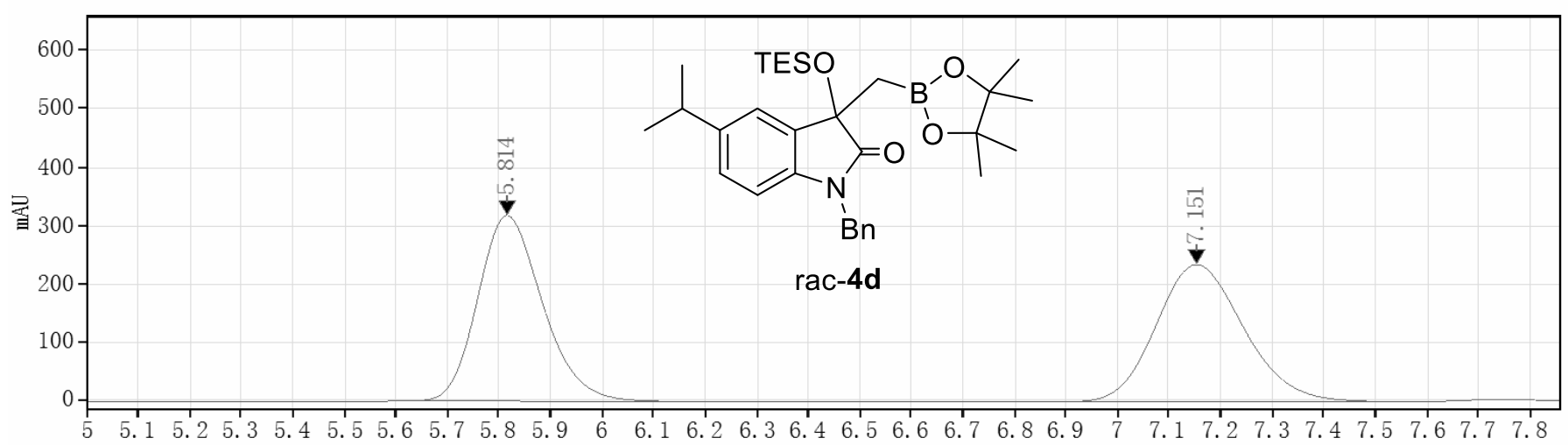

\begin{tabular}{ccccc}
\hline RetTime [min] & Type & Wide [min] & Area[mAu*s] & Height[mAu] \\
\hline 5.814 & MM m & 0.13 & 2764.31 & 317.46 \\
7.151 & MM m & 0.18 & 2765.60 & 234.47 \\
\hline
\end{tabular}

DAD1B, Sig=254, $4 \quad$ Ref=off

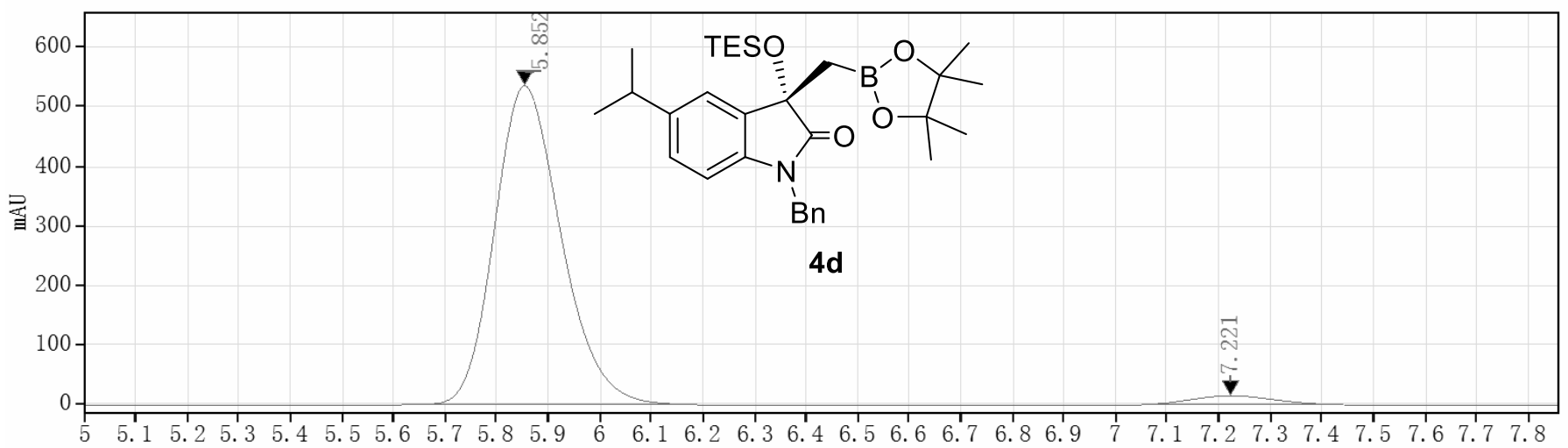

\begin{tabular}{|c|c|c|c|c|c|}
\hline RetTime [min] & Type & Wide [min] & Area[mAu*s] & Height[mAu] & Area $\%$ \\
\hline 5.852 & MM m & 0.14 & 4803.20 & 535.82 & 97.05 \\
\hline 7.221 & $\mathrm{MM} \mathrm{m}$ & 0.17 & 146.14 & 14.10 & 2.95 \\
\hline
\end{tabular}


DAD1B, Sig=254, $4 \quad$ Ref=off

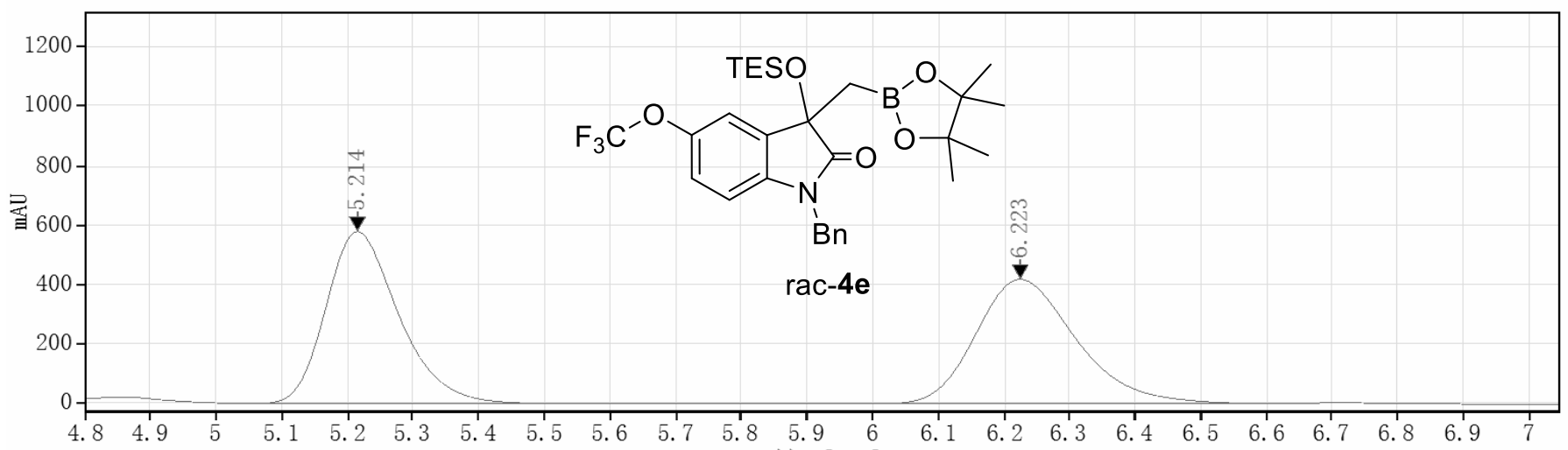

\begin{tabular}{ccccc}
\hline RetTime [min] & Type & Wide [min] & Area[mAu*s & Height[mAu] \\
\hline 5.214 & MM m & 0.12 & 4474.25 & 579.27 \\
6.223 & MM m & 0.16 & 4450.15 & 418.44 \\
\hline
\end{tabular}

DAD1B, Sig=254, $4 \quad$ Ref=off

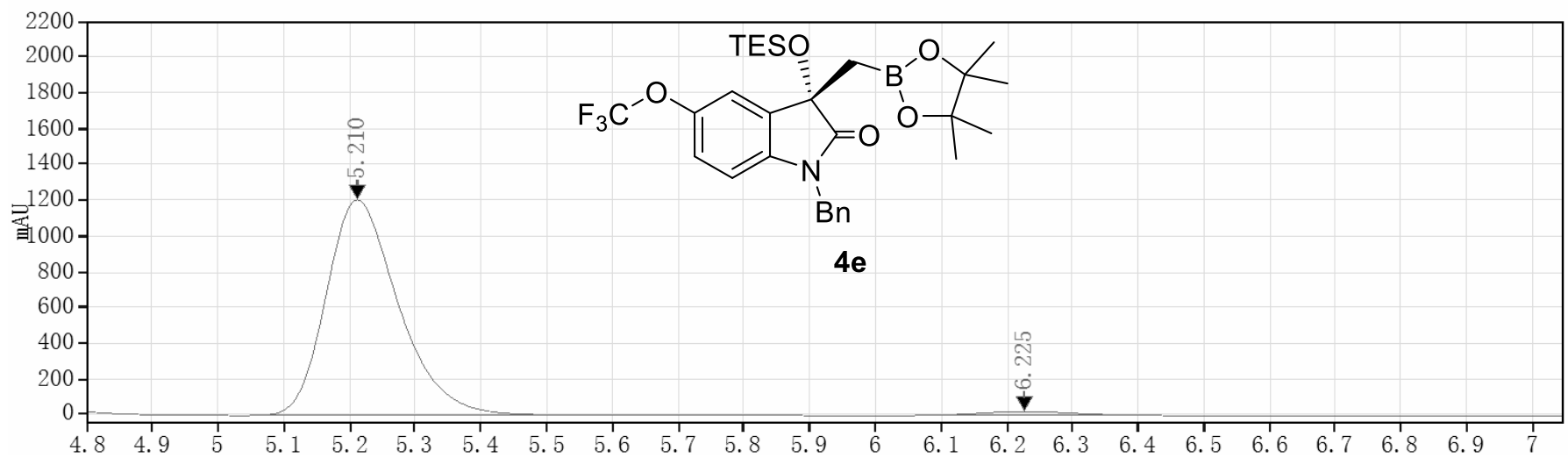

\begin{tabular}{ccccc}
\hline RetTime [min] & Type & Wide [min] & Area[mAu*s] & Height[mAu] \\
\hline 5.210 & MM m & 0.12 & 9185.28 & 1204.31 \\
6.225 & MM m & 0.15 & 197.49 & 21.39 \\
\hline
\end{tabular}


DAD1B, Sig=254, 4 Ref=off

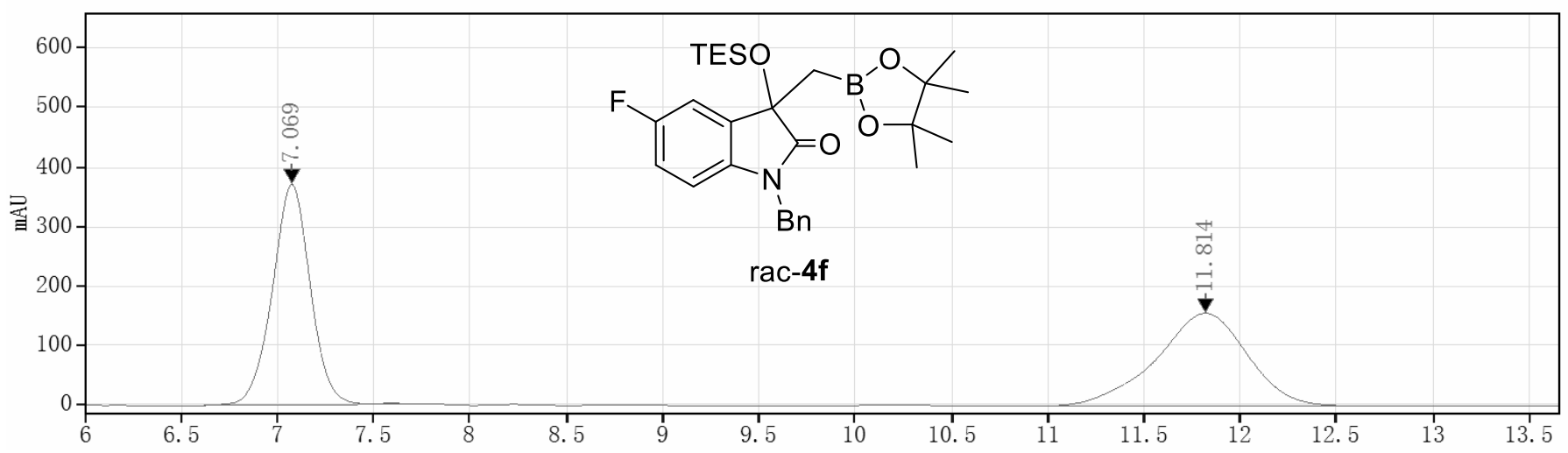

\begin{tabular}{ccccc}
\hline RetTime [min] & Type & Wide [min] & Area[mAu*s & Height[mAu] \\
\hline 7.069 & MM m & 0.21 & 5090.65 & 370.76 \\
11.814 & MM m & 0.50 & 5135.56 & 155.86 \\
\hline
\end{tabular}

DAD1B, Sig=254, 4 Ref=off

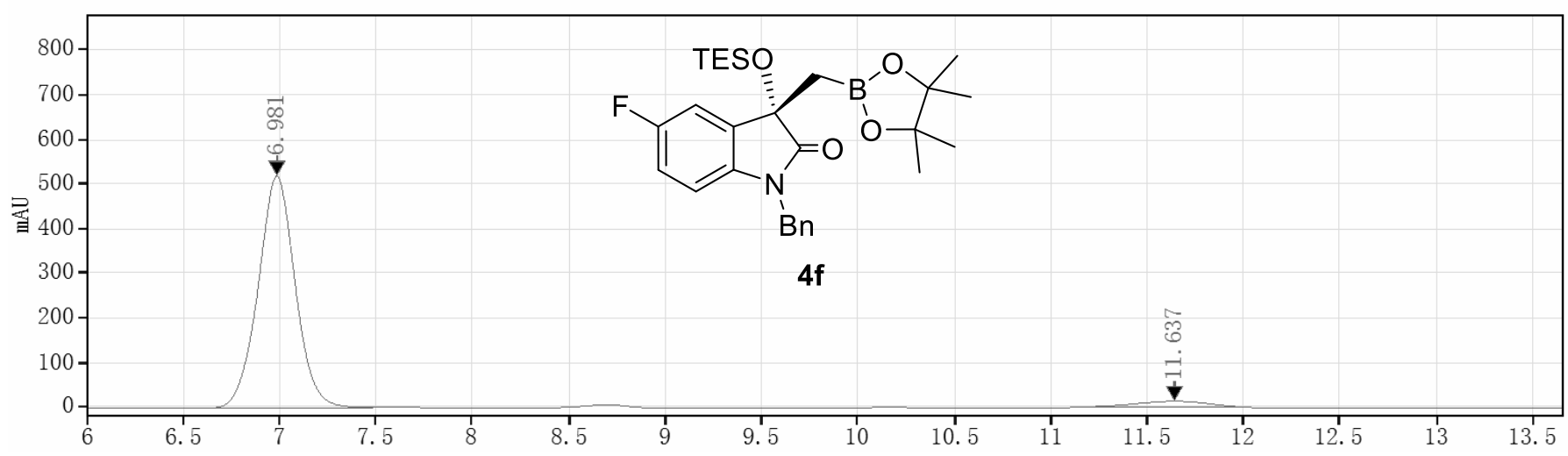

\begin{tabular}{ccccc}
\hline RetTime [min] & Type & Wide [min] & Area[mAu*s] & Height[mAu] \\
\hline 6.981 & MM m & 0.20 & 6910.92 & 518.72 \\
11.637 & MM m & 0.40 & 362.14 & 13.66 \\
\hline
\end{tabular}


DAD1B, Sig=254, 4 Ref=off

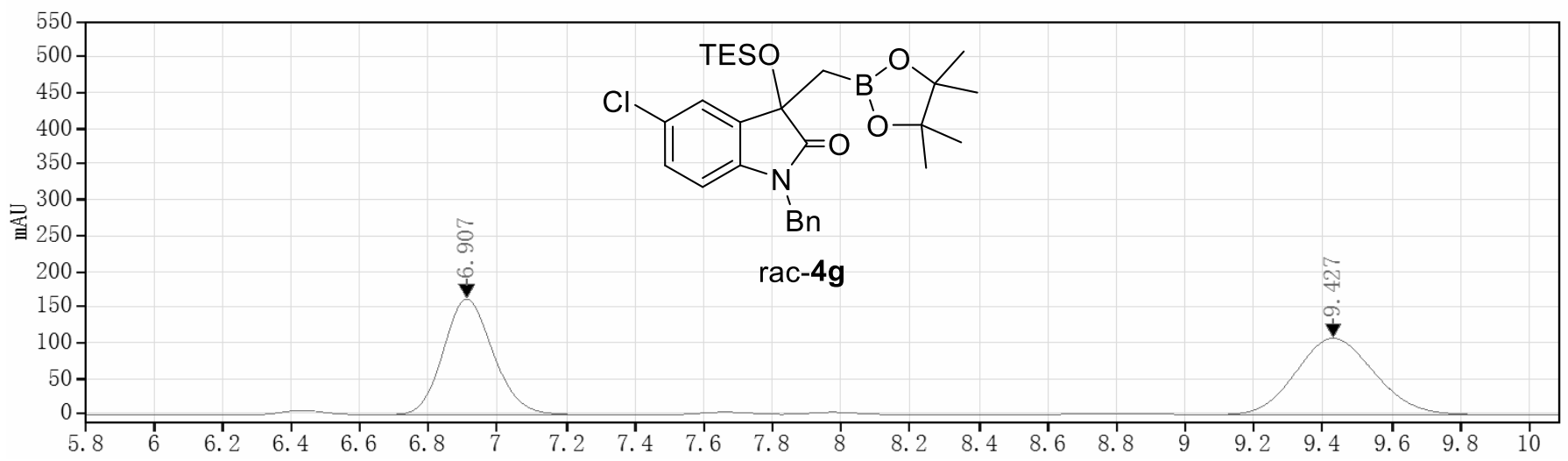

\begin{tabular}{ccccc}
\hline RetTime [min] & Type & Wide [min] & Area[mAu*s] & Height[mAu] \\
\hline 6.907 & MM m & 0.16 & 1637.11 & 161.90 \\
9.427 & MM m & 0.25 & 1696.73 & 106.84 \\
\hline
\end{tabular}

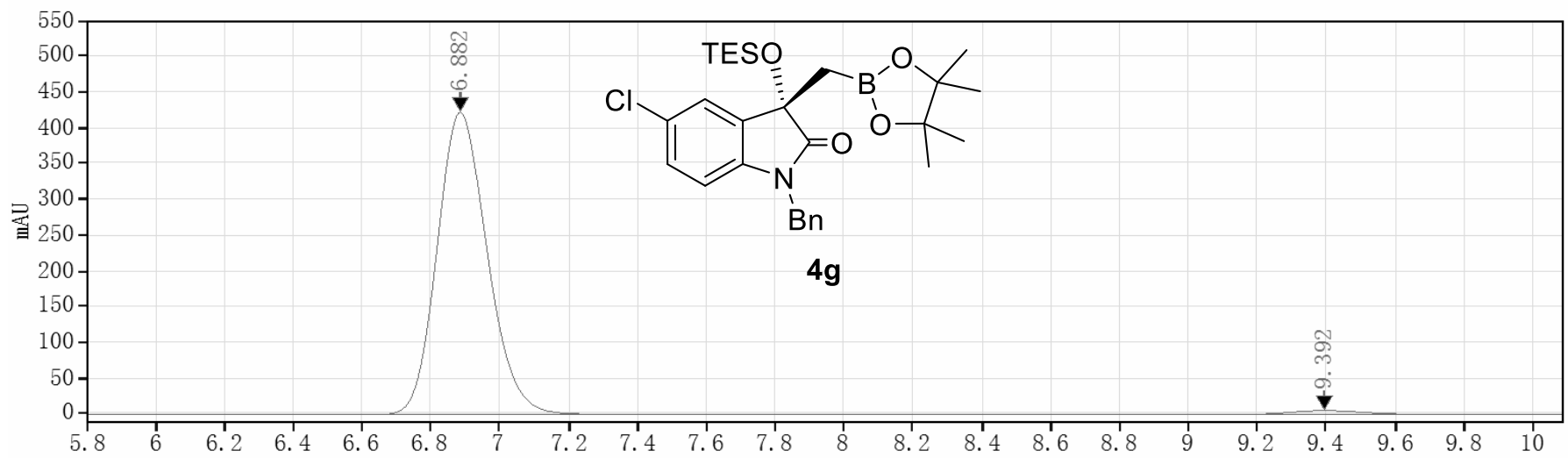

\begin{tabular}{ccccc}
\hline RetTime [min] & Type & Wide [min] & Area[mAu*s] & Height[mAu] \\
\hline 6.882 & MM m & 0.16 & 4267.00 & 422.66 \\
9.392 & MM m & 0.20 & 63.71 & 4.94 \\
\hline
\end{tabular}


DAD1B, Sig=254, 4 Ref=off

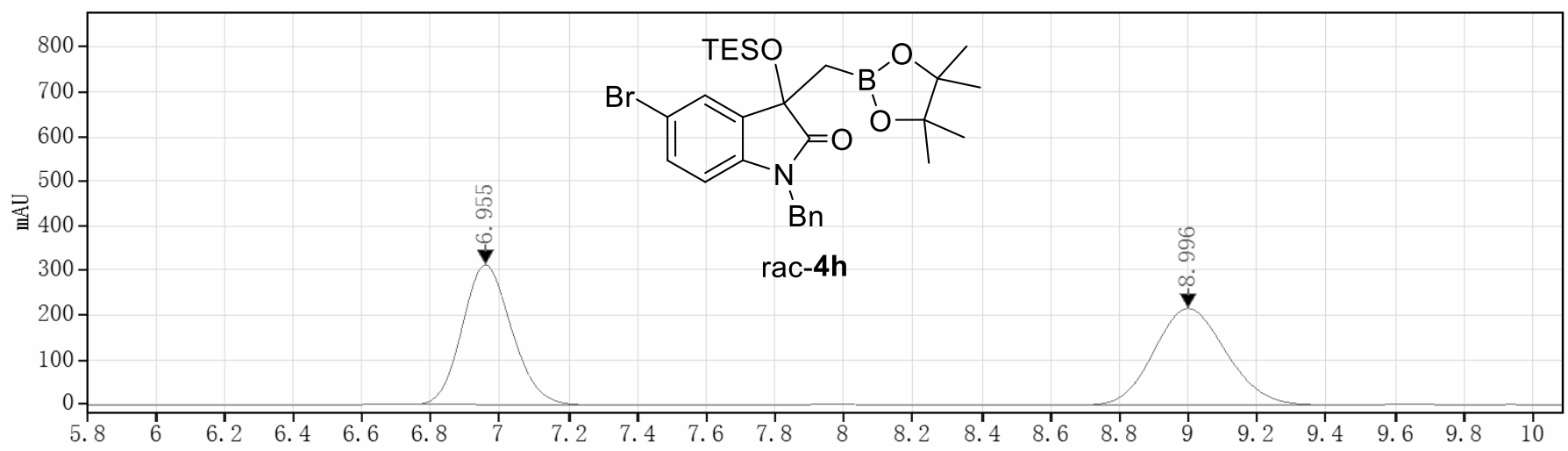

\begin{tabular}{ccccc}
\hline RetTime [min] & Type & Wide [min] & Area[mAu*s] & Height[mAu] \\
\hline 6.955 & MM m & 0.15 & 3122.28 & 312.80 \\
8.996 & MM m & 0.23 & 3150.18 & 216.15 \\
\hline
\end{tabular}

DAD1B, Sig=254, 4 Ref=off

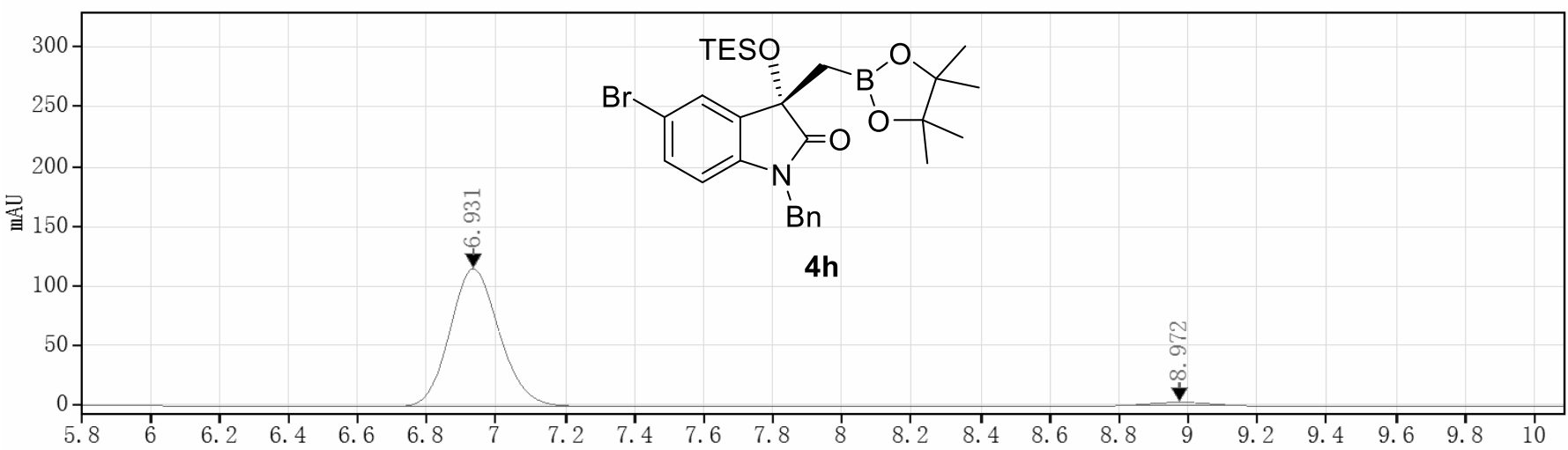

\begin{tabular}{ccccc}
\hline RetTime [min] & Type & Wide [min] & Area[mAu*s] & Height[mAu] \\
\hline 6.931 & MM m & 0.15 & 1143.03 & 115.14 \\
8.972 & MM m & 0.17 & 27.32 & 2.54 \\
\hline
\end{tabular}


DAD1B, Sig=254, 4 Ref=off

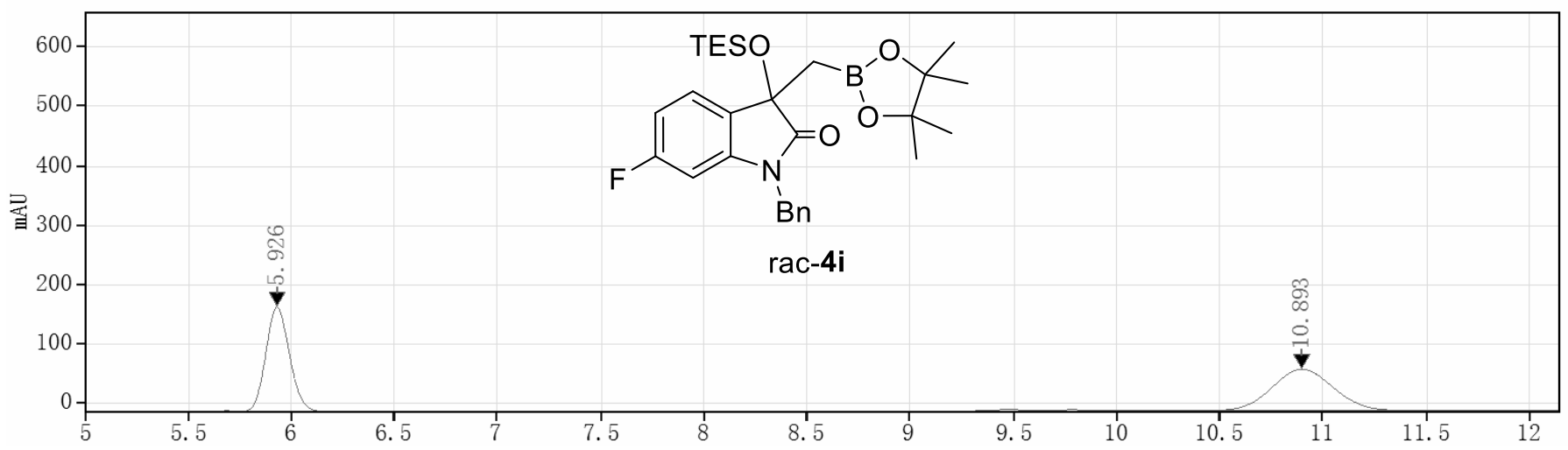

\begin{tabular}{|c|c|c|c|c|c|}
\hline RetTime [min] & Type & Wide [min] & Area[mAu*s] & Height[mAu] & Area $\%$ \\
\hline 5.926 & $\mathrm{MM} \mathrm{m}$ & 0.12 & 1397.58 & 175.81 & 49.29 \\
\hline 10.893 & $\mathrm{MM} \mathrm{m}$ & 0.32 & 1437.99 & 69.43 & 50.71 \\
\hline
\end{tabular}

DAD1B, Sig=254, $4 \quad$ Ref=off

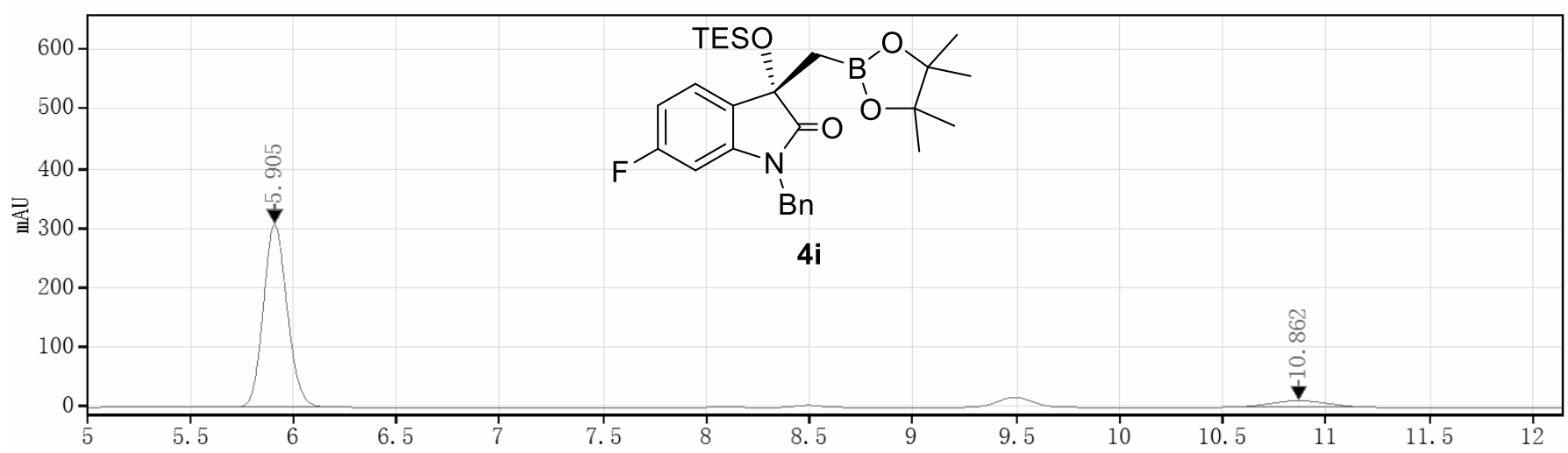

\begin{tabular}{|c|c|c|c|c|c|}
\hline RetTime [min] & Type & Wide [min] & Area[mAu*s] & Height[mAu] & Area $\%$ \\
\hline 5.905 & $\mathrm{MM} \mathrm{m}$ & 0.12 & 2463.15 & 307.04 & 92.93 \\
\hline 10.862 & $\mathrm{MM} \mathrm{m}$ & 0.29 & 187.31 & 10.24 & 7.07 \\
\hline
\end{tabular}


DAD1B, Sig=254, 4 Ref=off

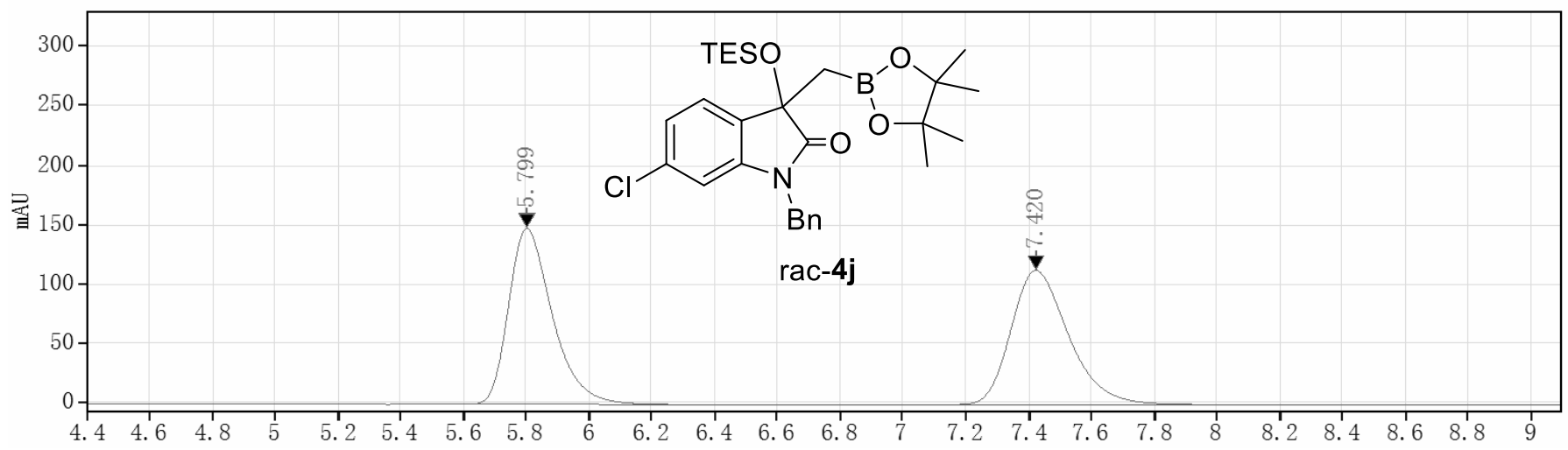

\begin{tabular}{ccccc}
\hline RetTime [min] & Type & Wide [min] & Area[mAu*s & Height[mAu] \\
\hline 5.799 & MM m & 0.15 & 1429.35 & 147.82 \\
7.420 & MM m & 0.20 & 1432.39 & 112.65 \\
\hline
\end{tabular}

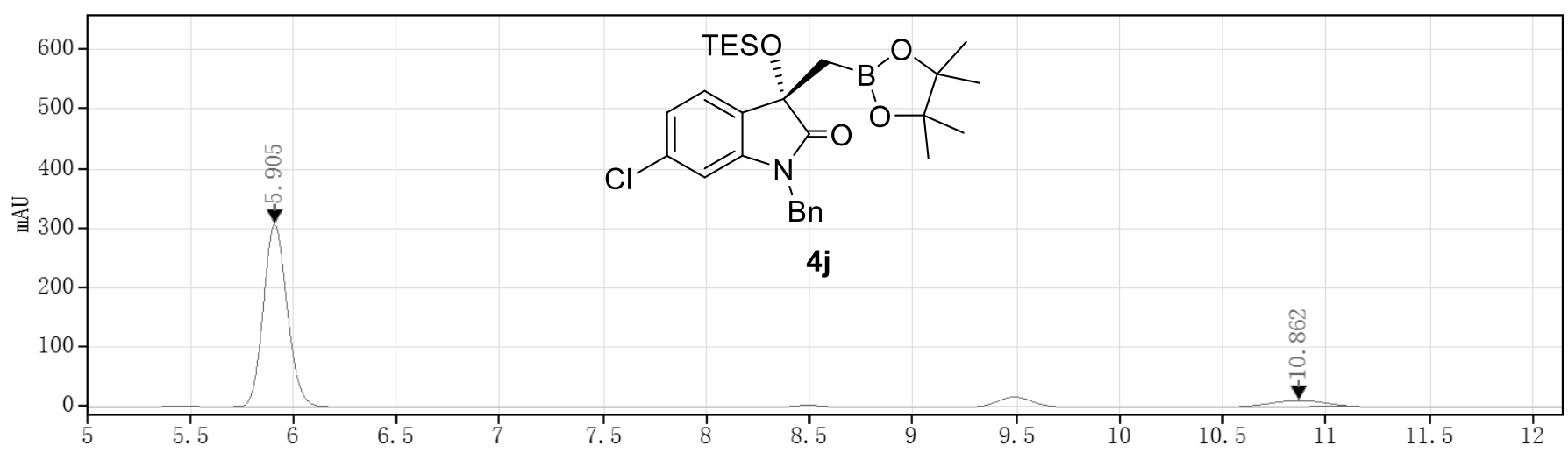

\begin{tabular}{ccccc}
\hline RetTime [min] & Type & Wide [min] & Area[mAu*s] & Height[mAu] \\
\hline 5.905 & MM m & 0.12 & 2463.15 & 307.04 \\
10.862 & MM m & 0.29 & 187.31 & 10.24 \\
\hline
\end{tabular}


DAD1B, Sig=254, 4 Ref=off

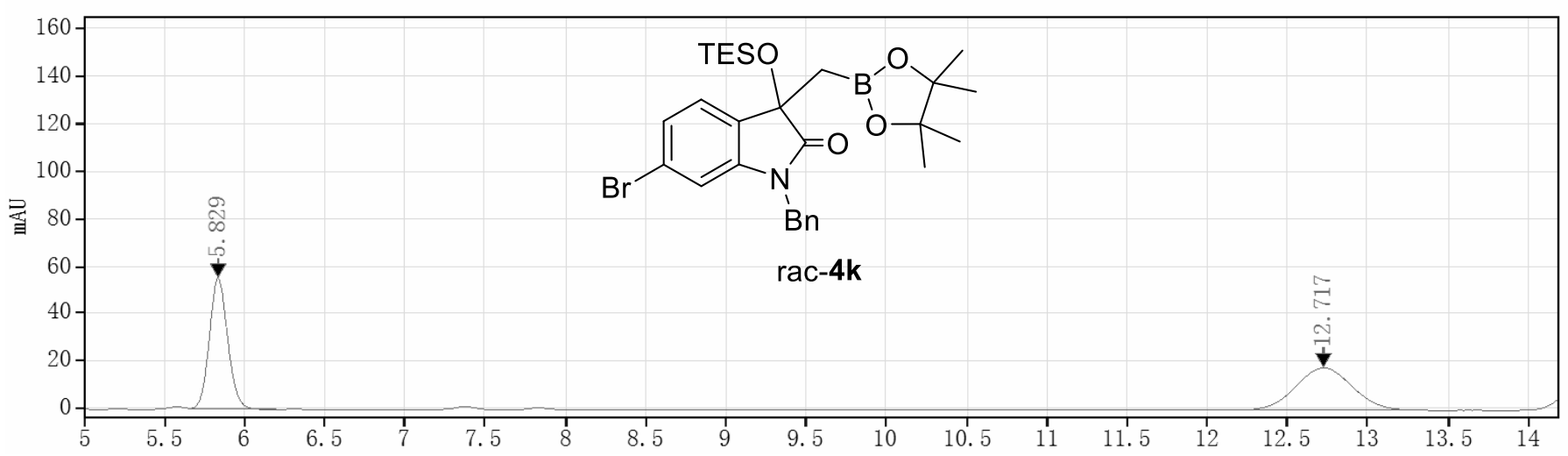

\begin{tabular}{cccccc}
\hline RetTime [min] & Type & Wide [min] & Area[mAu*s] & Height[mAu] & Area\% \\
\hline 5.829 & MM m & 0.12 & 432.09 & 54.76 & 49.94 \\
12.717 & MM m & 0.37 & 433.12 & 17.69 & 50.06 \\
\hline
\end{tabular}

DAD1B, Sig=254, $4 \quad$ Ref=off

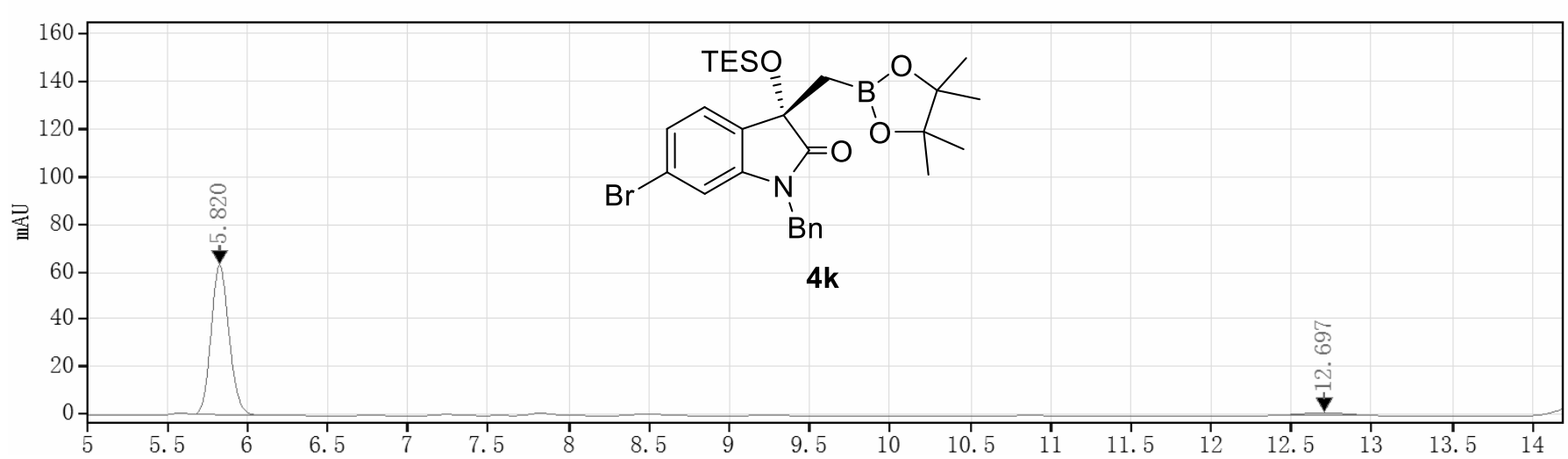

\begin{tabular}{ccccc}
\hline RetTime [min] & Type & Wide [min] & Area[mAu*s] & Height[mAu] \\
\hline 5.820 & MM m & 0.12 & 486.44 & 62.70 \\
12.697 & MM m & 0.24 & 22.87 & 1.14 \\
\hline
\end{tabular}


DAD1B, Sig=254, 4 Ref=off

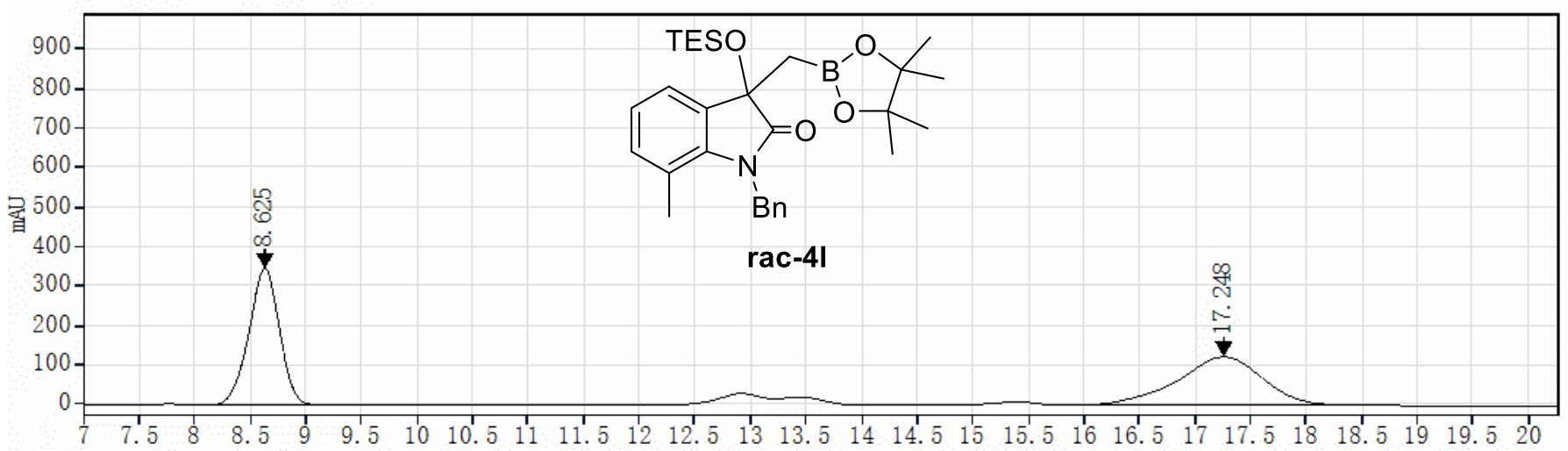

\begin{tabular}{ccccc}
\hline RetTime [min] & Type & Wide [min] & Area[mAu*s] & Height[mAu] \\
\hline 8.625 & MM m & 0.29 & 6647.49 & 345.59 \\
17.248 & MM m & 0.79 & 6551.84 & 122.50 \\
\hline
\end{tabular}

DAD1B, Sig=254, 4 Ref=off

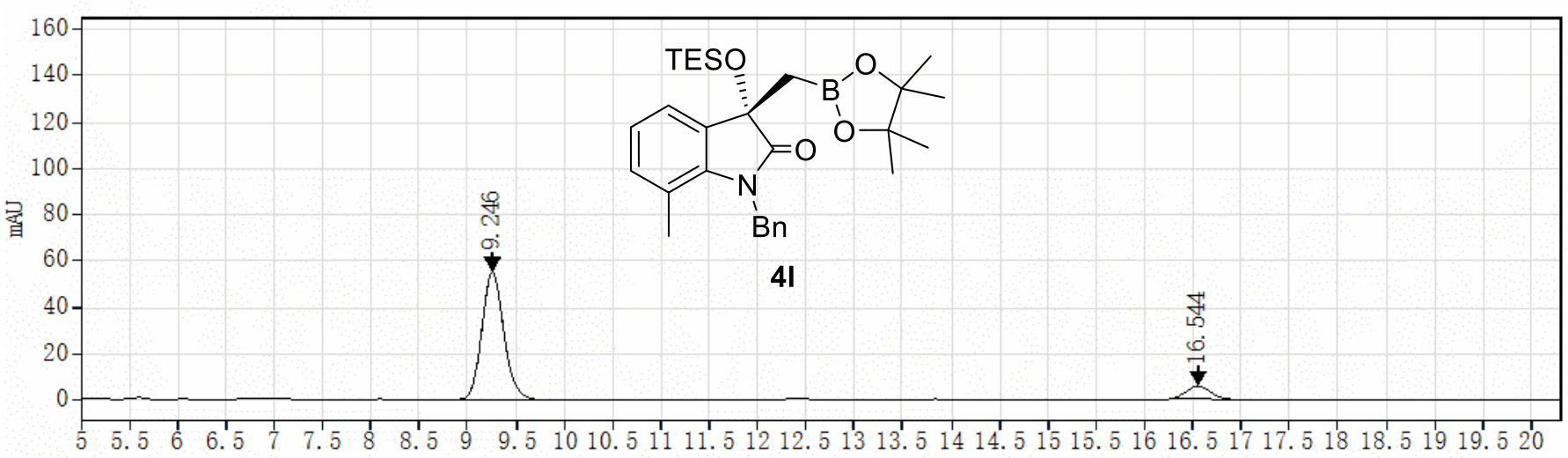

\begin{tabular}{ccccc}
\hline RetTime [min] & Type & Wide [min] & Area[mAu*s] & Height[mAu] \\
\hline 9.246 & MM m & 0.25 & 864.41 & 54.80 \\
16.544 & MM m & 0.26 & 96.00 & 5.54 \\
\hline
\end{tabular}


DAD1B, Sig=254, $4 \quad$ Ref=off

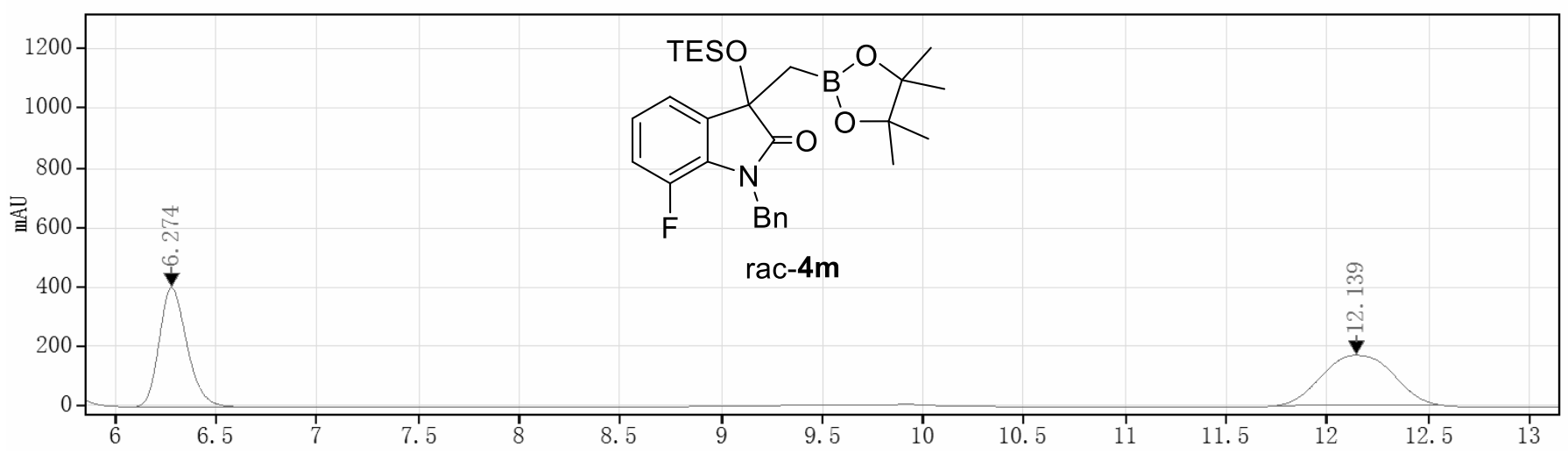

\begin{tabular}{ccccc}
\hline RetTime [min] & Type & Wide [min] & Area[mAu*s & Height[mAu] \\
\hline 6.274 & MM m & 0.15 & 3815.23 & 400.69 \\
12.139 & MM m & 0.40 & 4116.30 & 167.74 \\
\hline
\end{tabular}

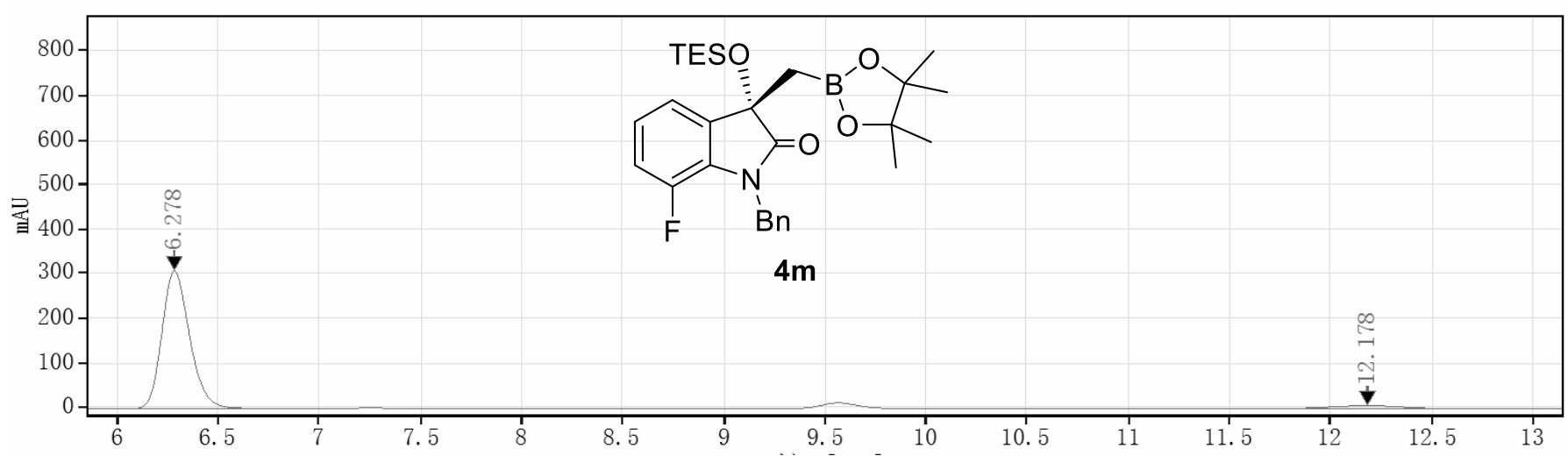

\begin{tabular}{ccccc}
\hline RetTime [min] & Type & Wide [min] & Area[mAu*s] & Height[mAu] \\
\hline 6.278 & MM m & 0.15 & 2932.21 & 308.42 \\
12.178 & MM m & 0.31 & 151.42 & 6.32 \\
\hline
\end{tabular}


DAD1B, Sig=254, 4 Ref=off

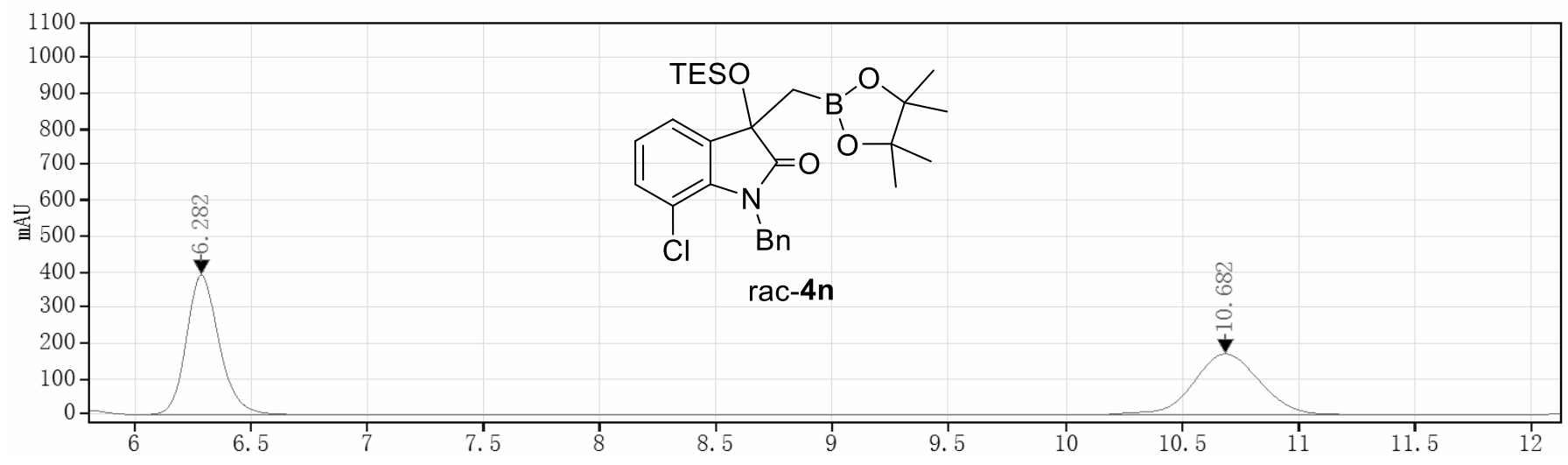

\begin{tabular}{ccccc}
\hline RetTime [min] & Type & Wide [min] & Area[mAu*s] & Height[mAu] \\
\hline 6.282 & MM m & 0.15 & 3644.38 & 391.85 \\
10.682 & MM m & 0.31 & 3636.45 & 171.23 \\
\hline
\end{tabular}

DAD1B, Sig=254, 4 Ref=off

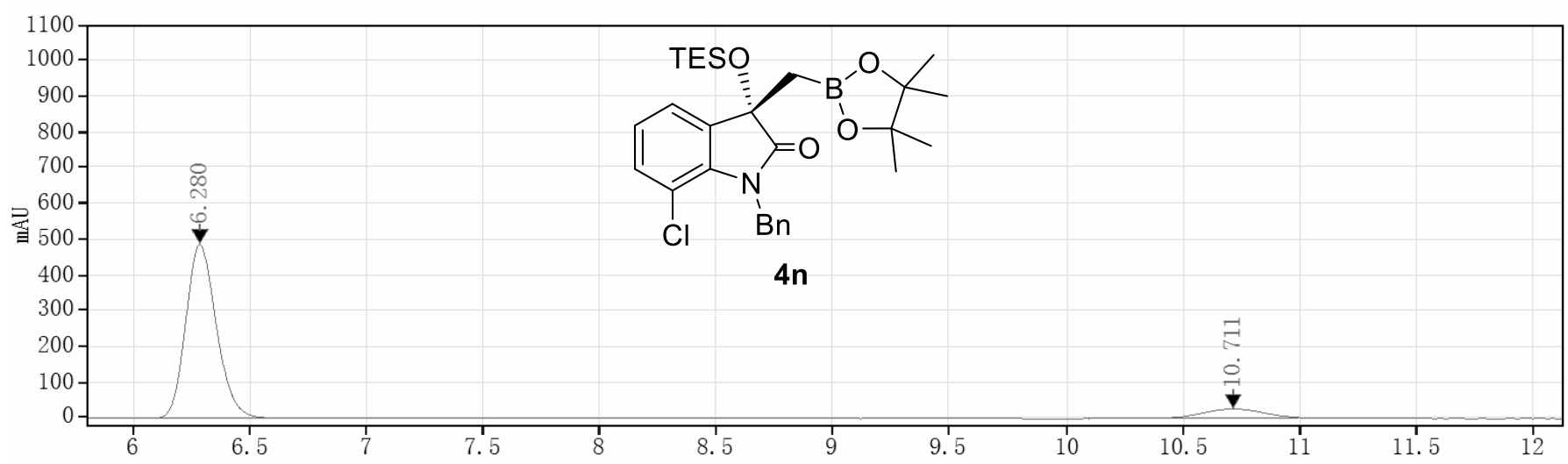

\begin{tabular}{ccccc}
\hline RetTime [min] & Type & Wide [min] & Area[mAu*s] & Height[mAu] \\
\hline 6.280 & MM m & 0.14 & 4446.72 & 487.00 \\
10.711 & MM m & 0.28 & 432.46 & 24.79 \\
\hline
\end{tabular}


DAD1B, Sig=254, 4 Ref=off

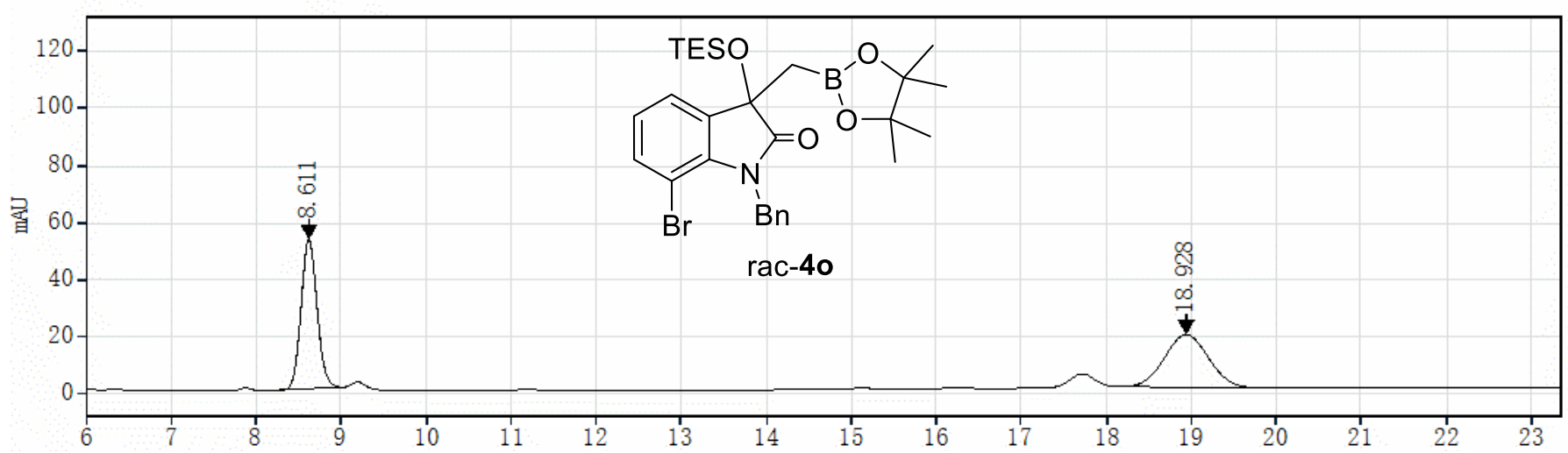

\begin{tabular}{ccccc}
\hline RetTime [min] & Type & Wide [min] & Area[mAu*s] & Height[mAu] \\
\hline 8.611 & MM m & 0.20 & 679.54 & 52.20 \\
18.928 & MM m & 0.53 & 655.80 & 18.32 \\
\hline
\end{tabular}

DAD1B, Sig=254, 4 Ref=off

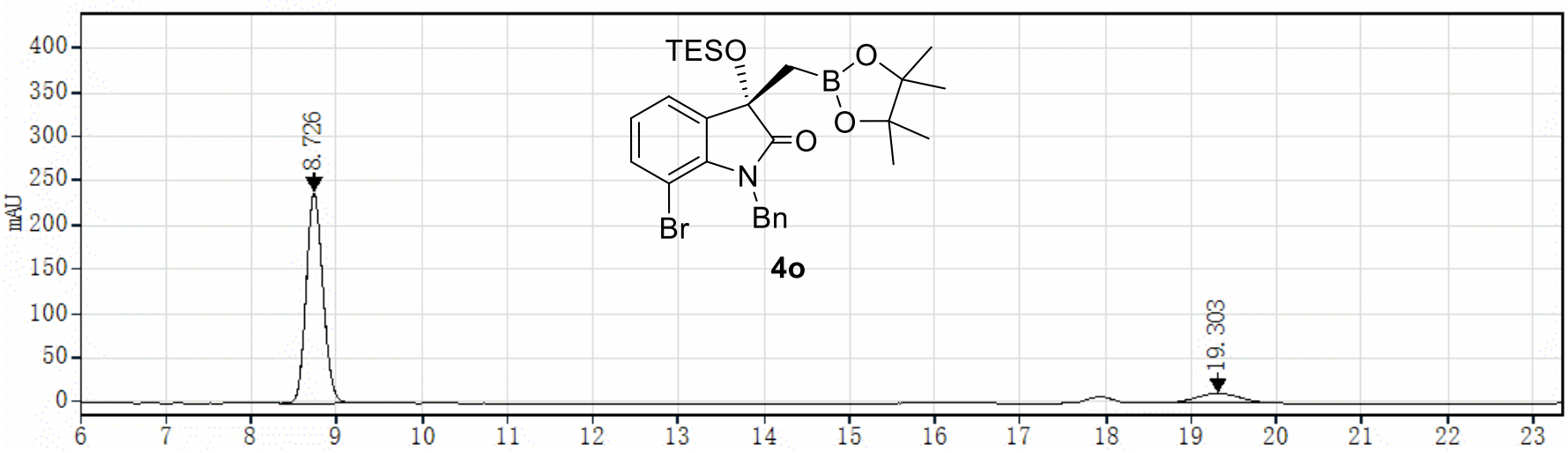

\begin{tabular}{|c|c|c|c|c|c|}
\hline RetTime [min] & Type & Wide [min] & Area[mAu*s] & Height[mAu] & Area $\%$ \\
\hline 8.726 & MM m & 0.21 & 3249.65 & 237.95 & 90.08 \\
\hline 19.303 & MM m & 0.45 & 357.70 & 10.35 & 9.92 \\
\hline
\end{tabular}


DAD1B, Sig=254, 4 Ref=off

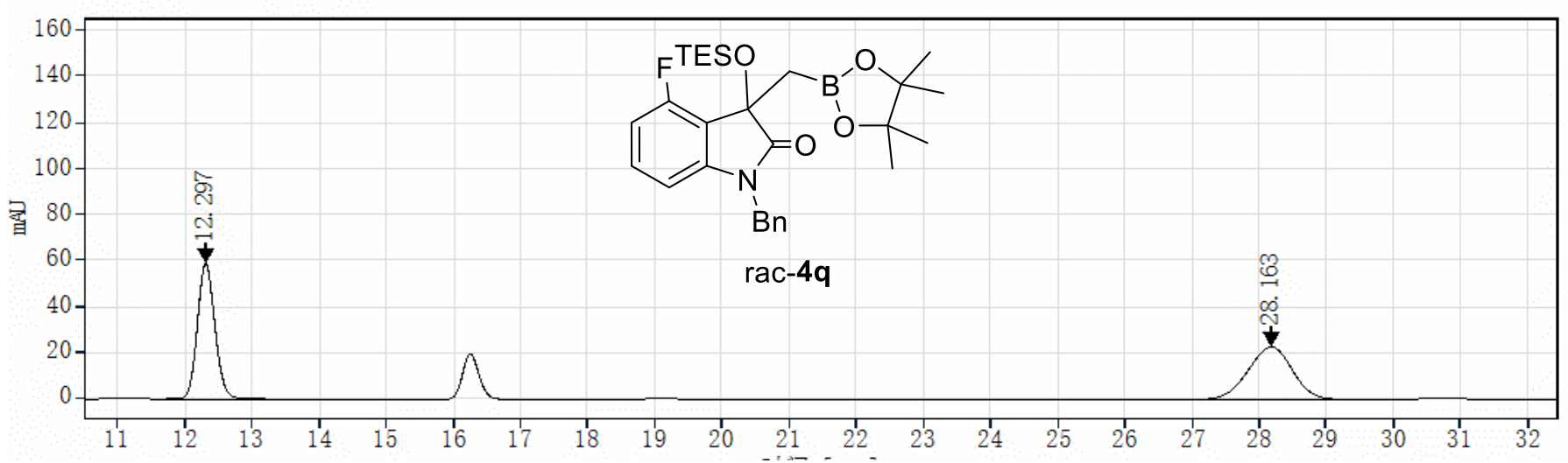

\begin{tabular}{ccccc}
\hline RetTime [min] & Type & Wide [min] & Area[mAu*s & Height[mAu] \\
\hline 12.297 & MM m & 0.28 & 1083.92 & 58.98 \\
28.163 & MM m & 0.64 & 1059.68 & 22.89 \\
\hline
\end{tabular}

DAD1B, Sig=254, 4 Ref=off

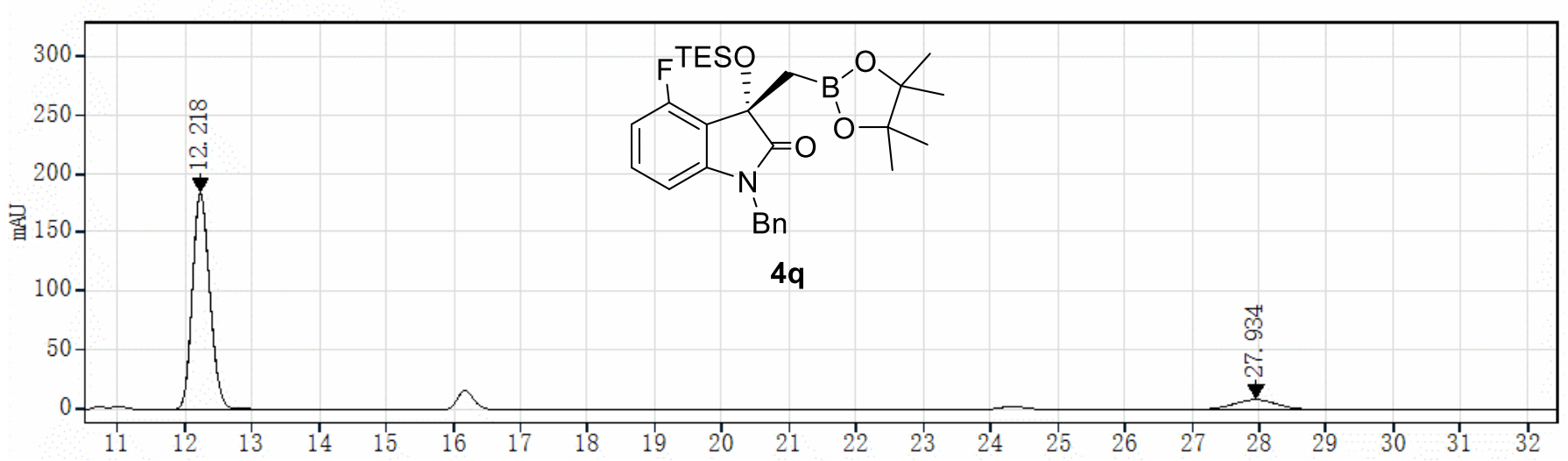

\begin{tabular}{cccccc}
\hline RetTime [min] & Type & Wide [min] & Area[mAu*s] & Height[mAu] & Area\% \\
\hline 12.218 & MM m & 0.28 & 3345.25 & 184.45 & 90.01 \\
27.934 & MM m & 0.53 & 371.26 & 8.28 & 9.99 \\
\hline
\end{tabular}


DAD1B, Sig=254, 4 Ref=off

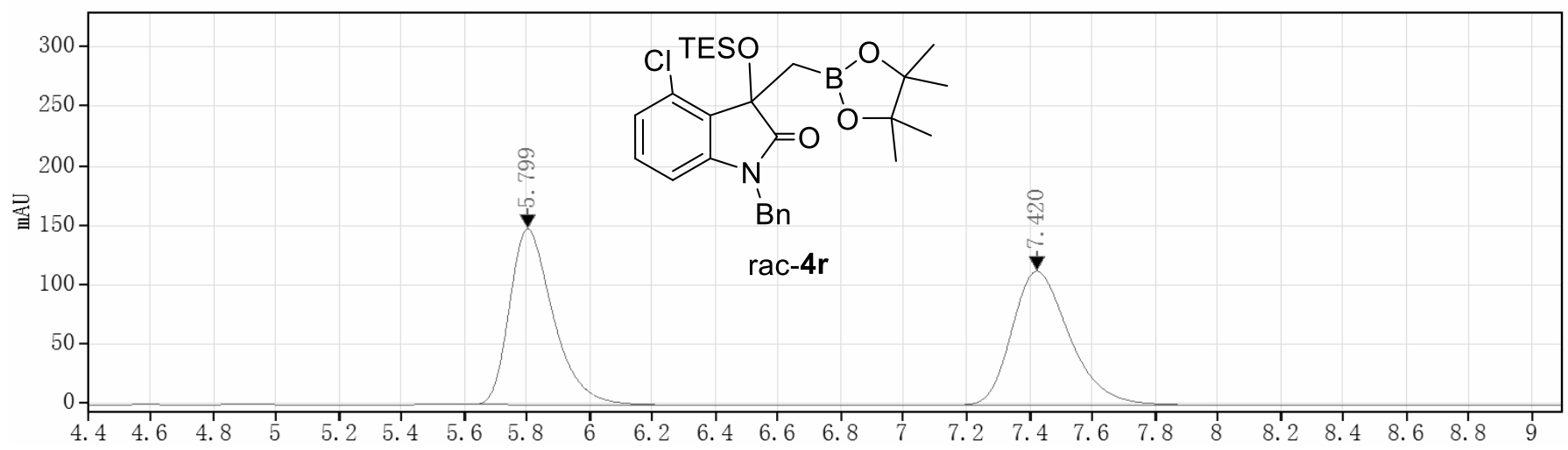

\begin{tabular}{ccccc}
\hline RetTime [min] & Type & Wide [min] & Area[mAu*s & Height[mAu] \\
\hline 5.799 & MM m & 0.15 & 1429.35 & 147.82 \\
7.420 & MM m & 0.20 & 1432.39 & 112.65 \\
\hline
\end{tabular}

DAD1B, Sig=254, 4 Ref=of $f$

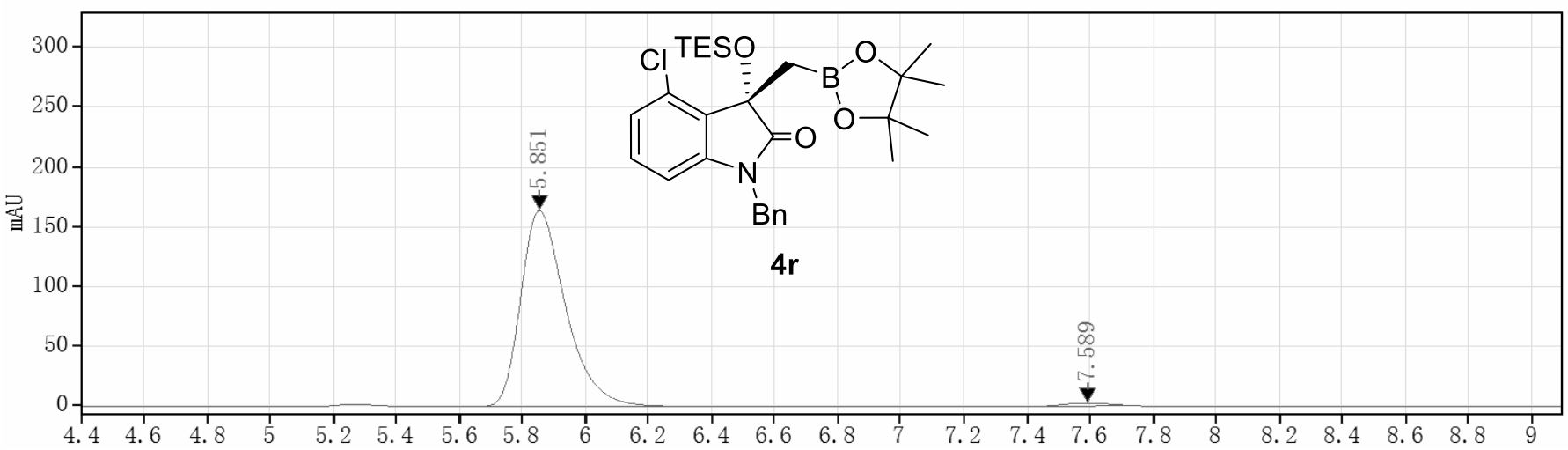

\begin{tabular}{ccccc}
\hline RetTime [min] & Type & Wide [min] & Area[mAu*s] & Height[mAu] \\
\hline 5.851 & MM m & 0.15 & 1631.18 & 164.18 \\
7.589 & MM m & 0.16 & 24.99 & 2.53 \\
\hline
\end{tabular}


DAD1B, Sig=254, $4 \quad$ Ref=off

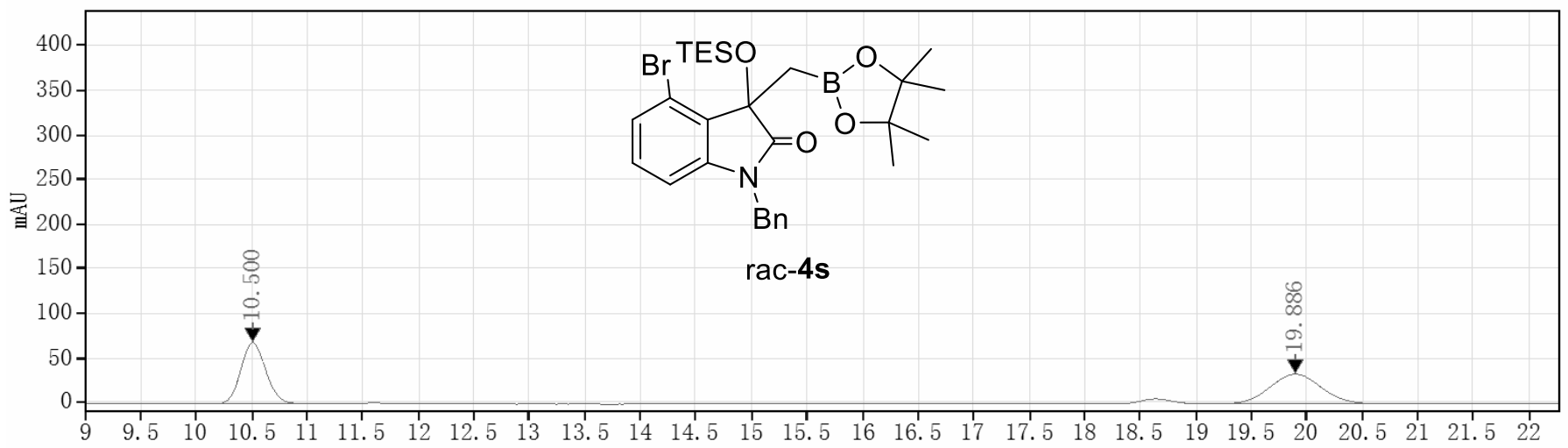

\begin{tabular}{cccccc}
\hline RetTime [min] & Type & Wide [min] & Area[mAu*s] & Height[mAu] & Area\% \\
\hline 10.500 & MM m & 0.24 & 1065.13 & 68.26 & 49.70 \\
19.886 & MM m & 0.49 & 1077.88 & 33.27 & 50.30 \\
\hline
\end{tabular}

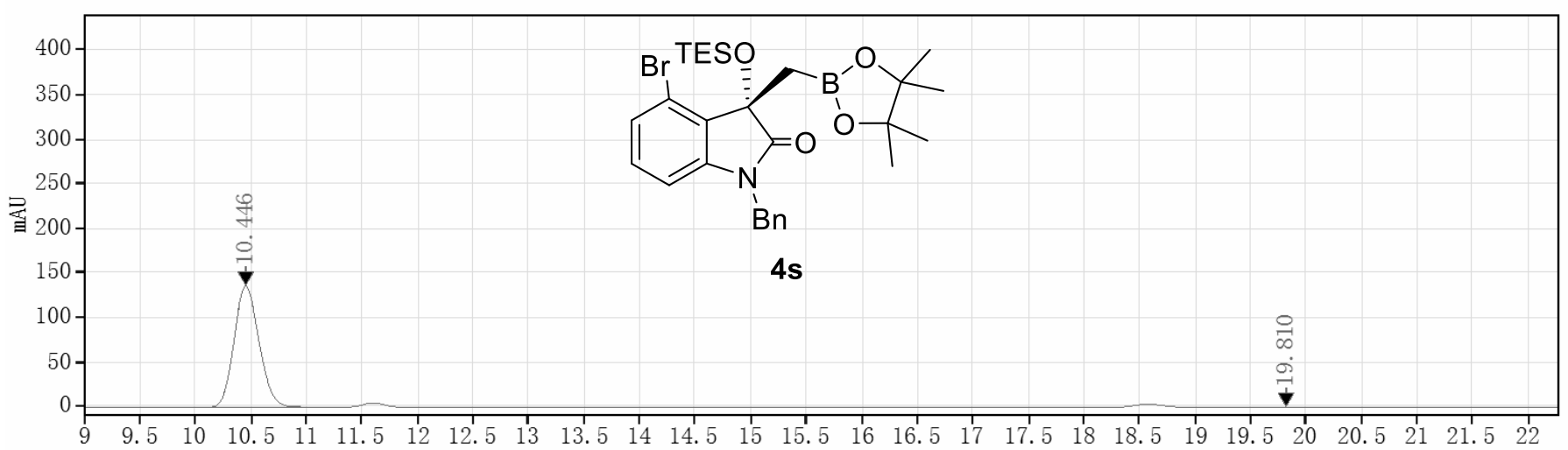

\begin{tabular}{ccccc}
\hline RetTime [min] & Type & Wide [min] & Area[mAu*s] & Height[mAu] \\
\hline 10.446 & MM m & 0.24 & 2130.51 & 135.71 \\
19.810 & MM m & 0.24 & 1.68 & 0.08 \\
\hline
\end{tabular}


DAD1B, Sig=254, 4 Ref=off

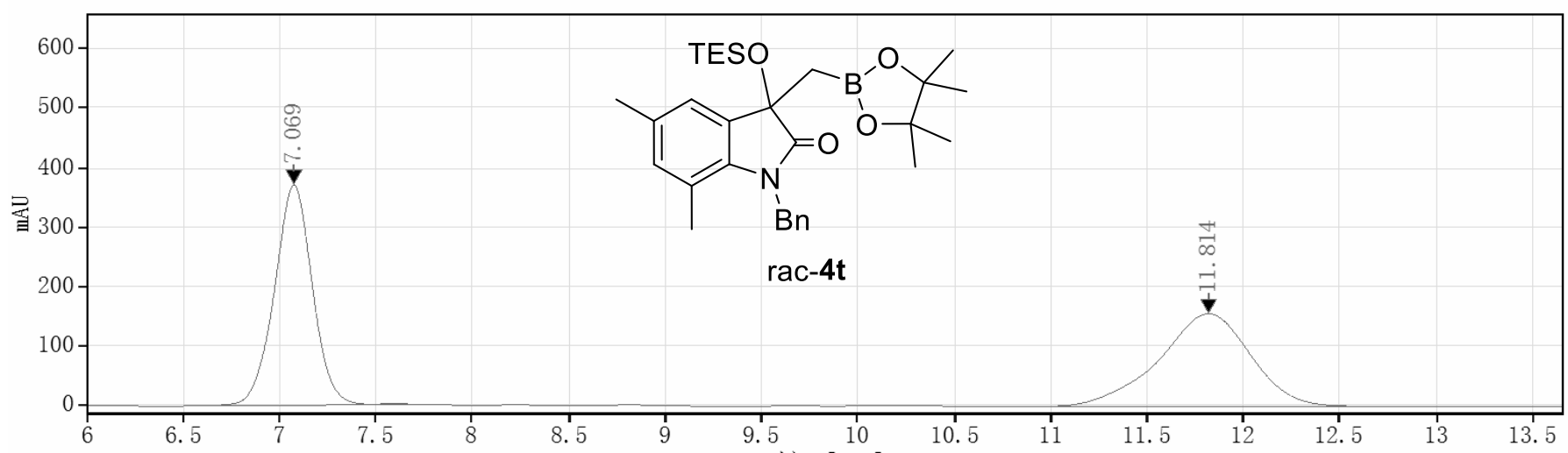

\begin{tabular}{ccccc}
\hline RetTime [min] & Type & Wide [min] & Area[mAu*s & Height[mAu] \\
\hline 7.069 & MM m & 0.21 & 5090.65 & 370.76 \\
11.814 & MM m & 0.50 & 5135.56 & 155.86 \\
\hline
\end{tabular}

DAD1B, Sig=254, $4 \quad$ Ref=off

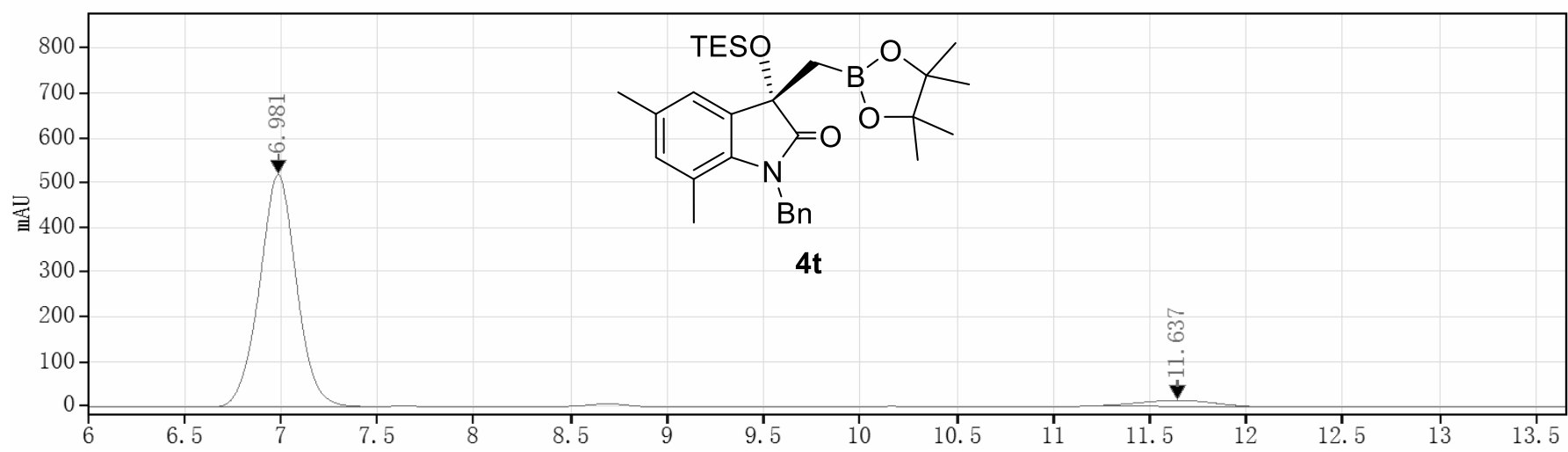

\begin{tabular}{ccccc}
\hline RetTime [min] & Type & Wide [min] & Area[mAu*s] & Height[mAu] \\
\hline 6.981 & MM m & 0.20 & 6910.92 & 518.72 \\
11.637 & MM m & 0.40 & 362.14 & 13.66 \\
\hline
\end{tabular}


DAD1B, Sig=254, 4 Ref=off

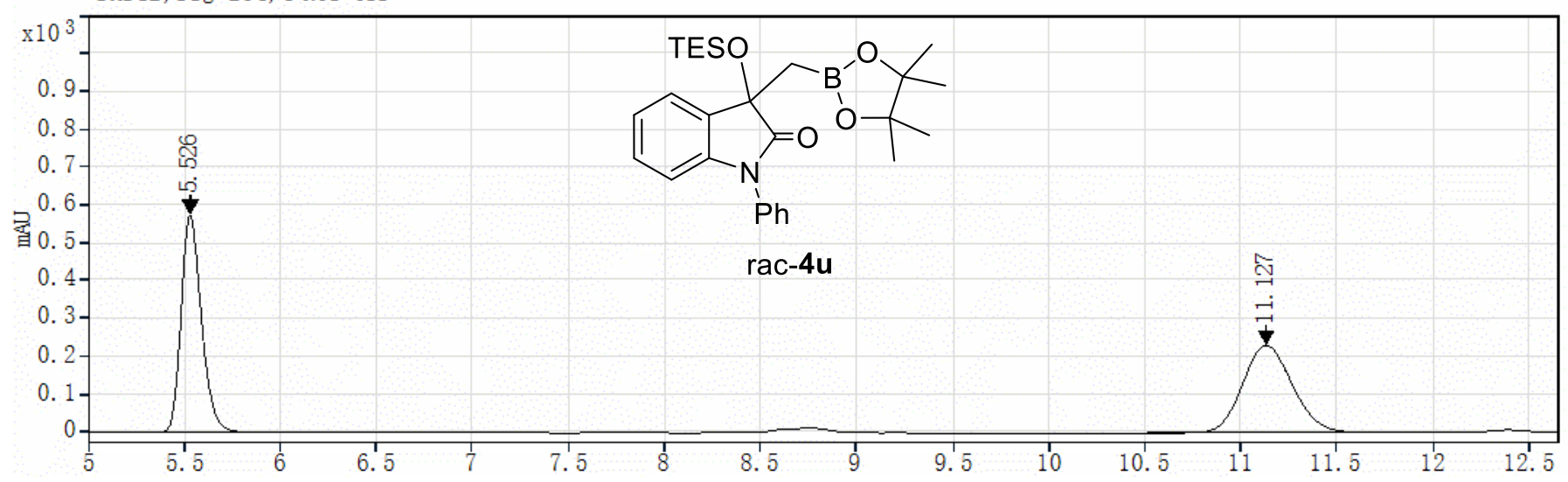

\begin{tabular}{|c|c|c|c|c|c|}
\hline RetTime [min] & Type & Wide [min] & Area[mAu*s] & Height[mAu] & Area $\%$ \\
\hline 5.526 & MM m & 0.11 & 4218.97 & 574.27 & 50.20 \\
\hline 11.127 & $\mathrm{MM} \mathrm{m}$ & 0.28 & 4185.67 & 231.31 & 49.80 \\
\hline
\end{tabular}

DAD1B, Sig=254, 4 Ref=off

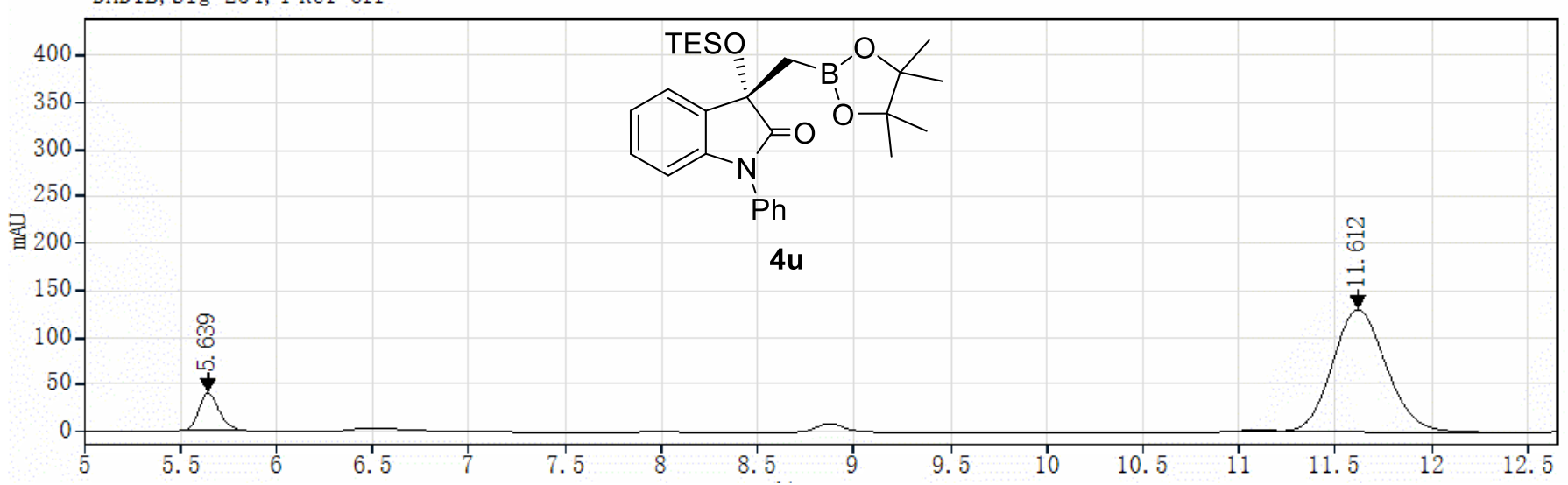

\begin{tabular}{|c|c|c|c|c|c|}
\hline RetTime [min] & Type & Wide [min] & Area[mAu*s] & Height[mAu] & Area\% \\
\hline 5.639 & $\mathrm{MM} \mathrm{m}$ & 0.11 & 285.43 & 39.91 & 9.98 \\
\hline 11.612 & $\mathrm{MM} \mathrm{m}$ & 0.30 & 2572.92 & 130.58 & 90.02 \\
\hline
\end{tabular}


DAD1B, Sig=254, 4 Ref=off

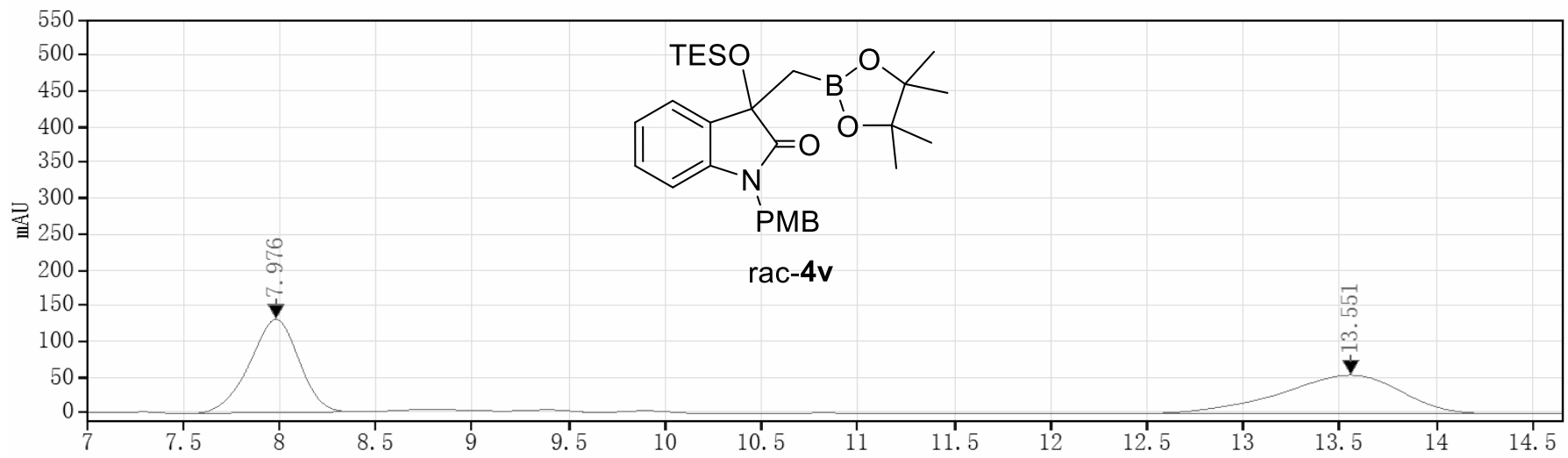

\begin{tabular}{ccccc}
\hline RetTime [min] & Type & Wide [min] & Area[mAu*s & Height[mAu] \\
\hline 7.976 & MM m & 0.27 & 2321.07 & 130.32 \\
13.551 & MM m & 0.64 & 2358.95 & 54.60 \\
\hline
\end{tabular}

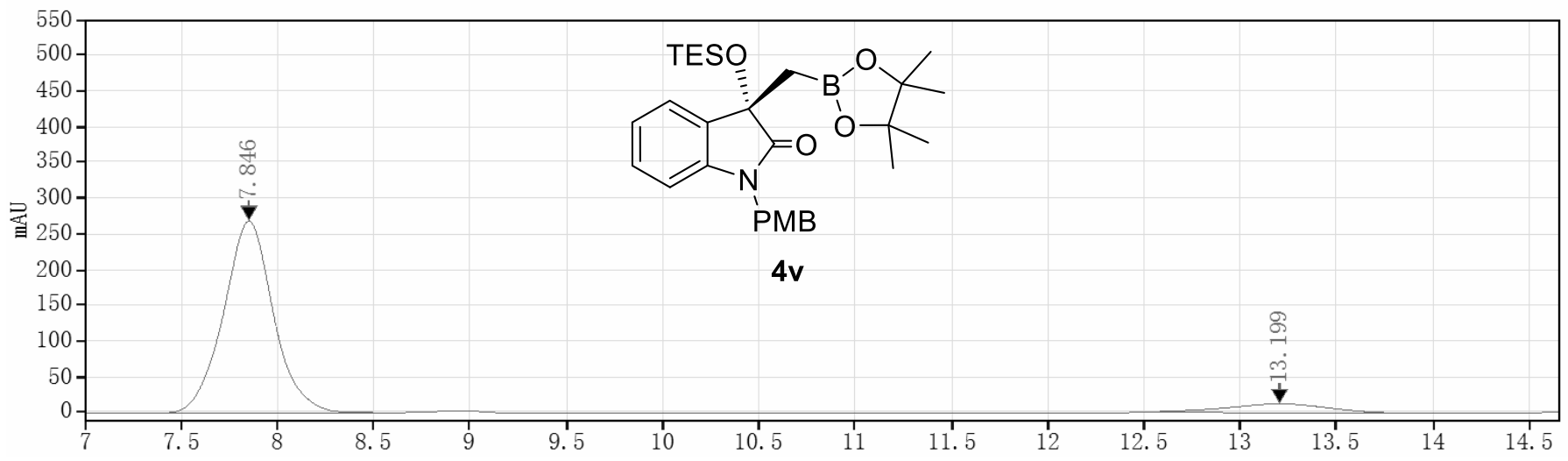

\begin{tabular}{ccccc}
\hline RetTime [min] & Type & Wide [min] & Area[mAu*s] & Height[mAu] \\
\hline 7.846 & MM m & 0.27 & 4821.58 & 268.45 \\
13.199 & MM m & 0.50 & 446.84 & 12.26 \\
\hline
\end{tabular}




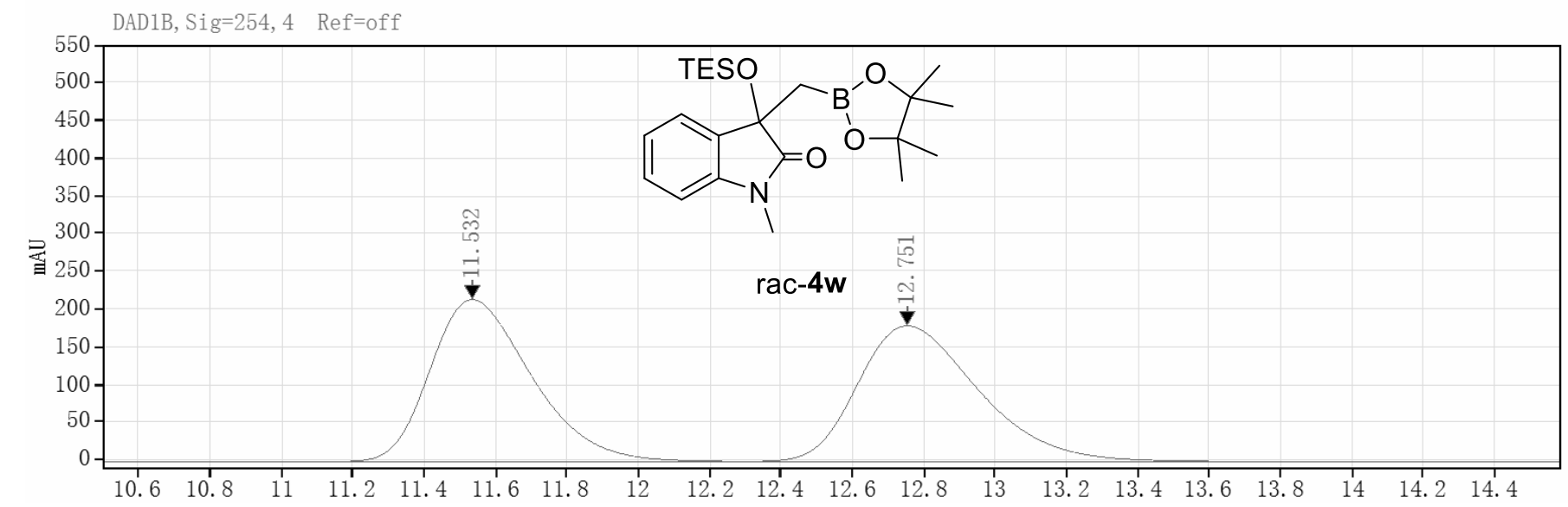

\begin{tabular}{cccccc}
\hline RetTime [min] & Type & Wide $[\mathrm{min}]$ & Area[mAu*s] & Height[mAu] & Area\% \\
\hline 11.532 & MM m & 0.30 & 4238.97 & 214.86 & 50.00 \\
12.751 & MM m & 0.36 & 4239.61 & 179.95 & 50.00 \\
\hline
\end{tabular}

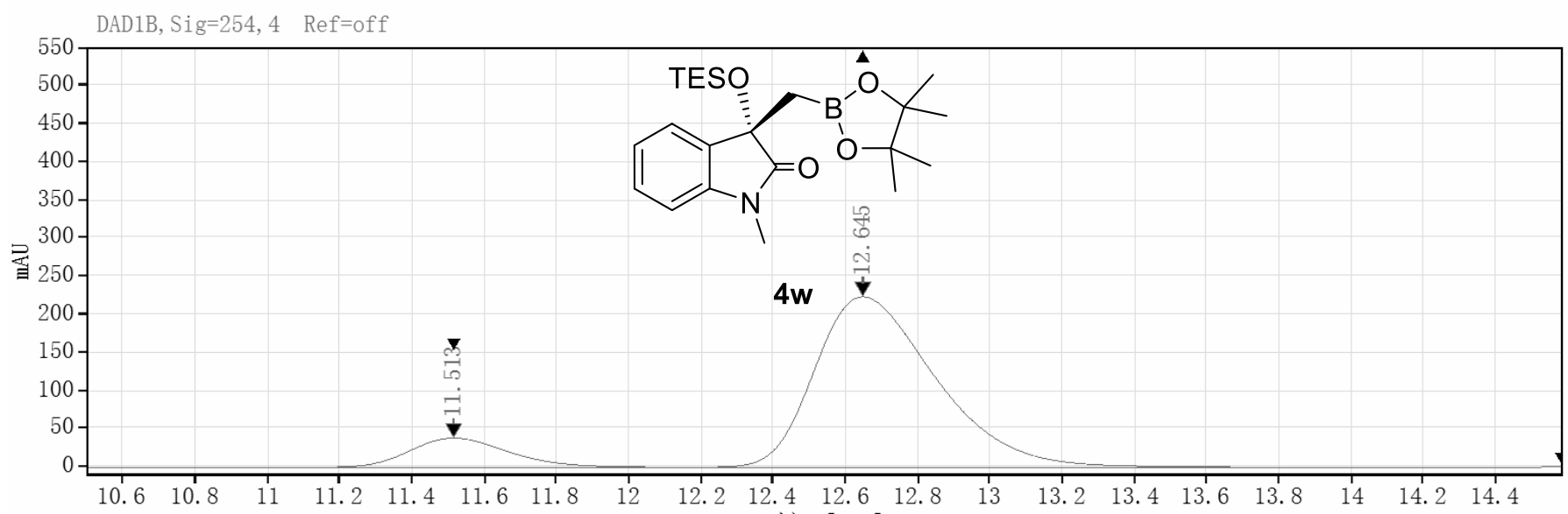

\begin{tabular}{cccccc}
\hline RetTime $[\mathrm{min}]$ & Type & Wide $[\mathrm{min}]$ & Area[mAu*s] & Height[mAu] & Area\% \\
\hline 11.513 & BB & 1.10 & 731.17 & 38.33 & 12.28 \\
12.645 & BB & 2.00 & 5222.41 & 223.80 & 87.72 \\
\hline
\end{tabular}


DAD1B, Sig=254, 4 Ref=off

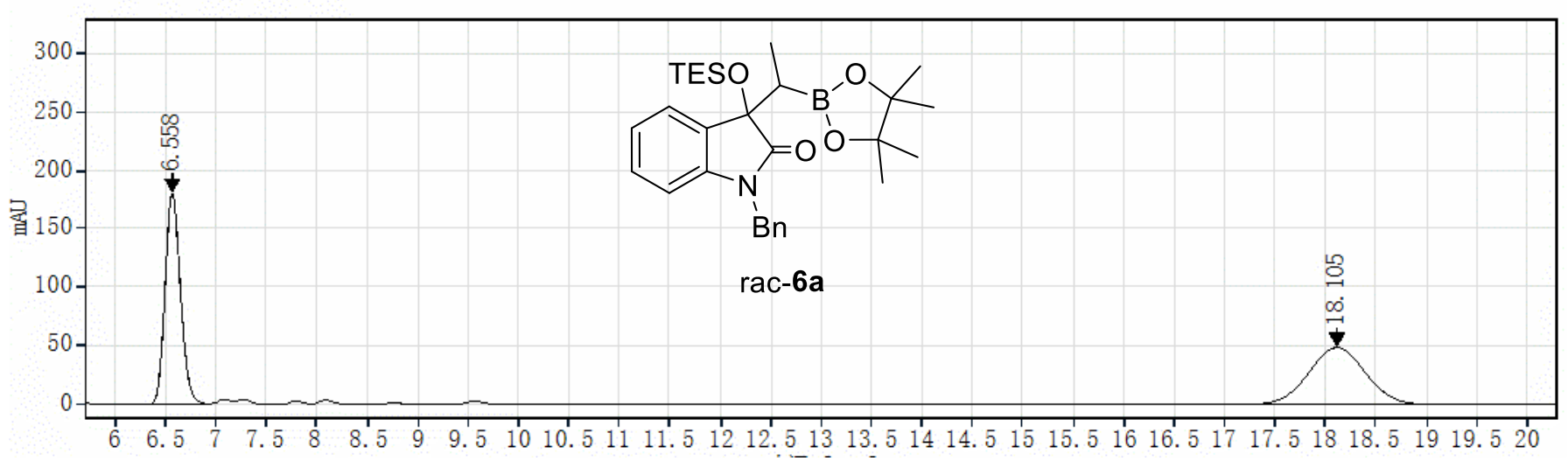

\begin{tabular}{ccccc}
\hline RetTime [min] & Type & Wide [min] & Area[mAu*s & Height[mAu] \\
\hline 6.558 & MM m & 0.16 & 1921.57 & 180.74 \\
18.105 & MM m & 0.61 & 1932.44 & 49.86 \\
\hline
\end{tabular}

DAD1B, Sig=254, 4 Ref=off

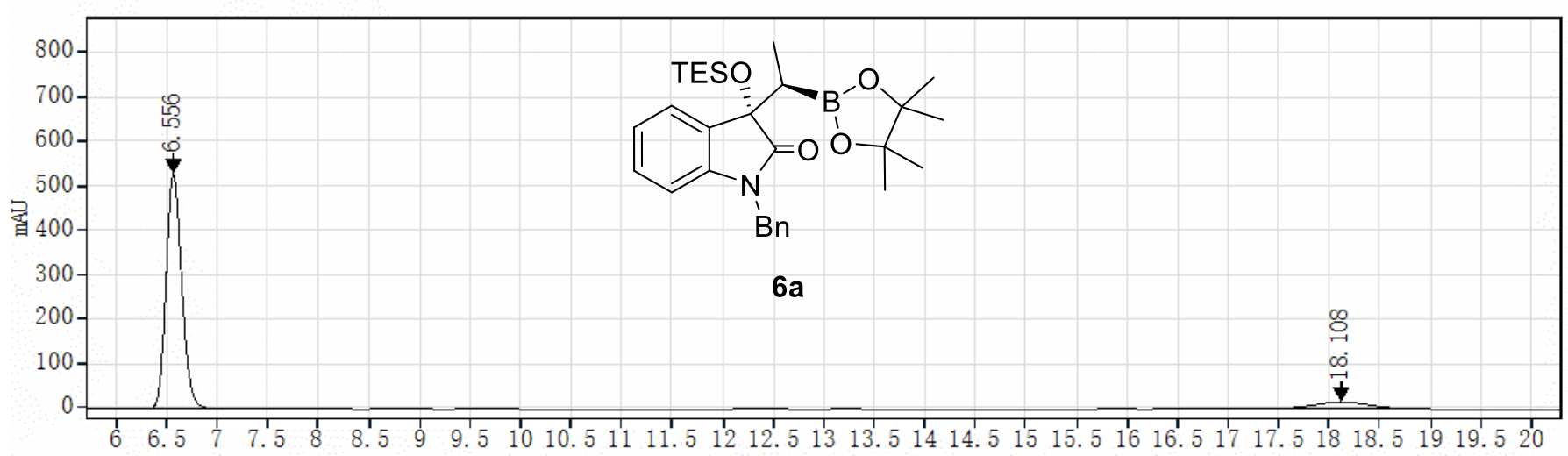

\begin{tabular}{ccccc}
\hline RetTime [min] & Type & Wide [min] & Area[mAu*s] & Height[mAu] \\
\hline 6.556 & MM m & 0.17 & 5711.80 & 528.76 \\
18.108 & MM m & 0.45 & 443.27 & 13.33 \\
\hline
\end{tabular}


DAD1B, Sig=254, 4 Ref=off

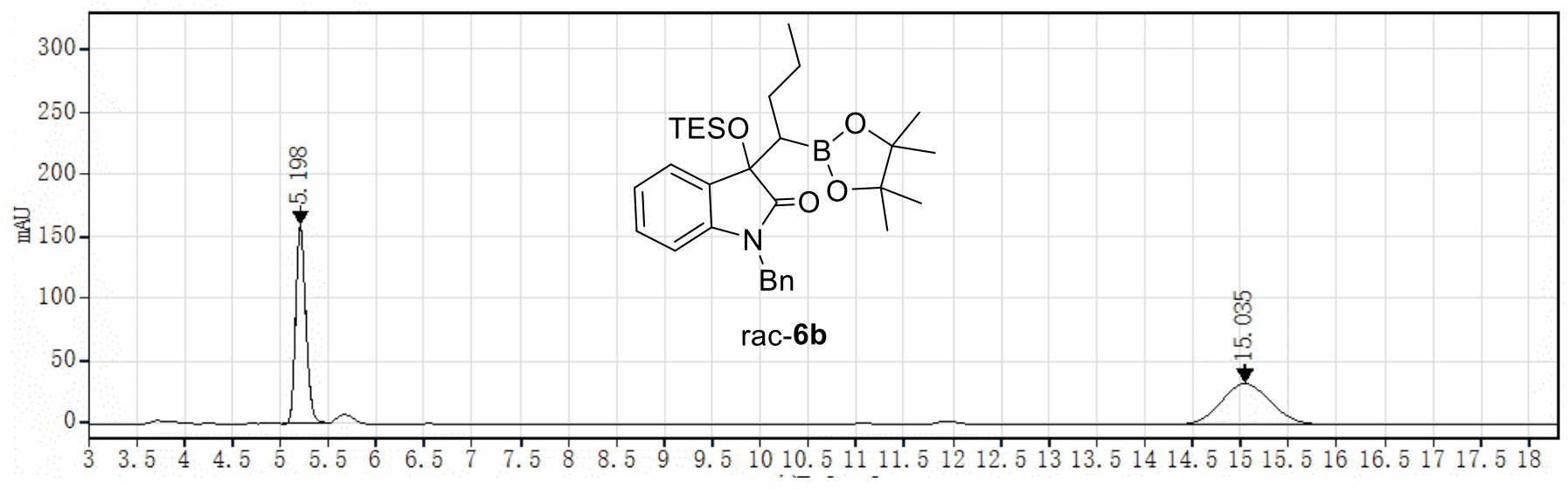

\begin{tabular}{ccccc}
\hline RetTime [min] & Type & Wide [min] & Area[mAu*s & Height[mAu] \\
\hline 5.198 & MM m & 0.11 & 1165.16 & 158.99 \\
15.035 & MM m & 0.54 & 1174.48 & 32.77 \\
\hline
\end{tabular}

DAD1B, Sig=254, 4 Ref=off

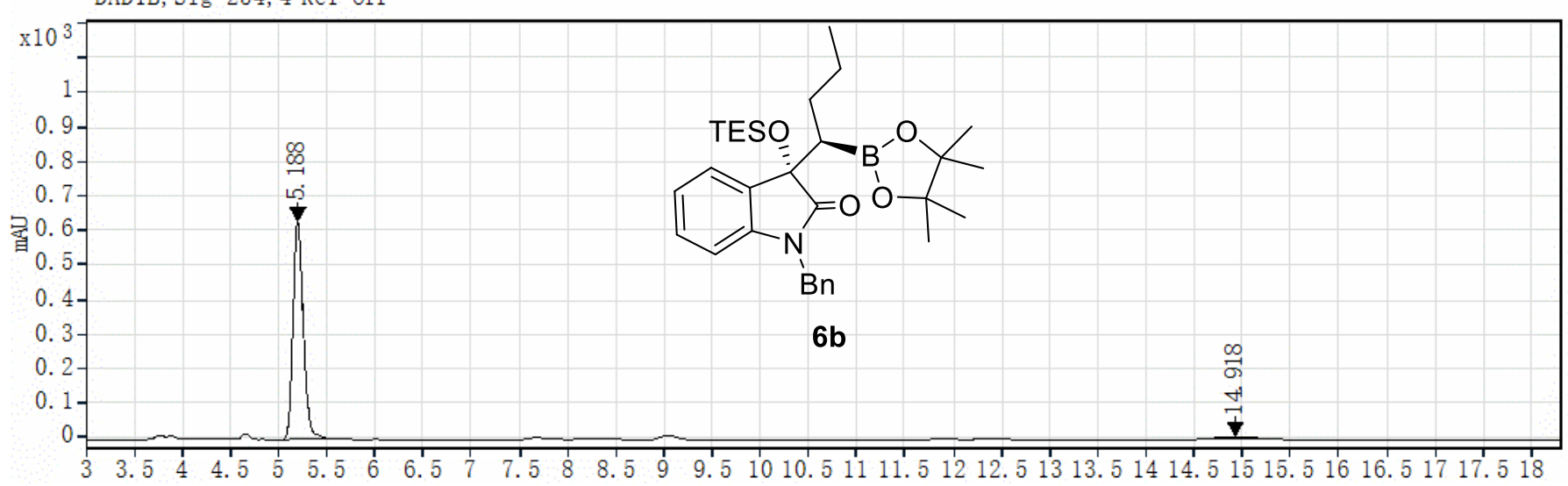

\begin{tabular}{cccccc}
\hline RetTime $[\mathrm{min}]$ & Type & Wide $[\mathrm{min}]$ & Area[mAu*s] & Height[mAu] & Area\% \\
\hline 5.188 & $\mathrm{MM} \mathrm{m}$ & 0.11 & 4675.89 & 629.15 & 96.43 \\
14.918 & $\mathrm{MM} \mathrm{m}$ & 0.32 & 173.06 & 6.41 & 3.57 \\
\hline
\end{tabular}


DAD1B, Sig=254, 4 Ref=off

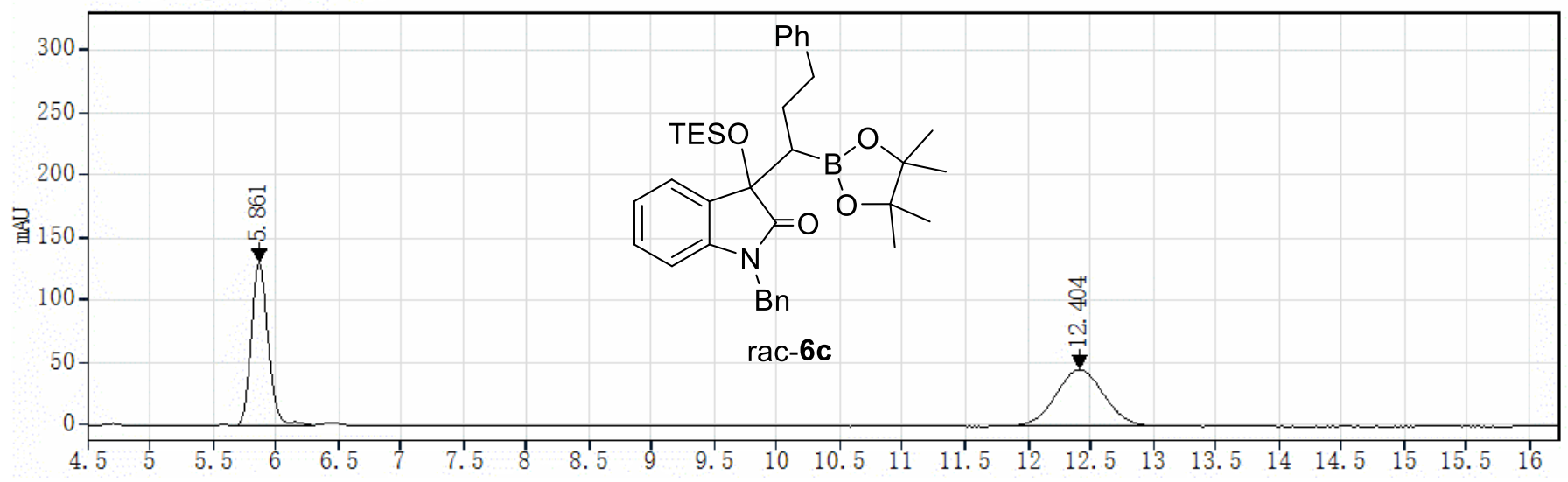

\begin{tabular}{|c|c|c|c|c|c|}
\hline RetTime [min] & Type & Wide [min] & Area[mAu*s] & Height[mAu] & Area $\%$ \\
\hline 5.861 & $\mathrm{MM} \mathrm{m}$ & 0.14 & 1211.15 & 129.81 & 49.99 \\
\hline 12.404 & $\mathrm{MM} \mathrm{m}$ & 0.41 & 1211.51 & 45.40 & 50.01 \\
\hline
\end{tabular}

DAD1B, Sig=254, 4 Ref=off

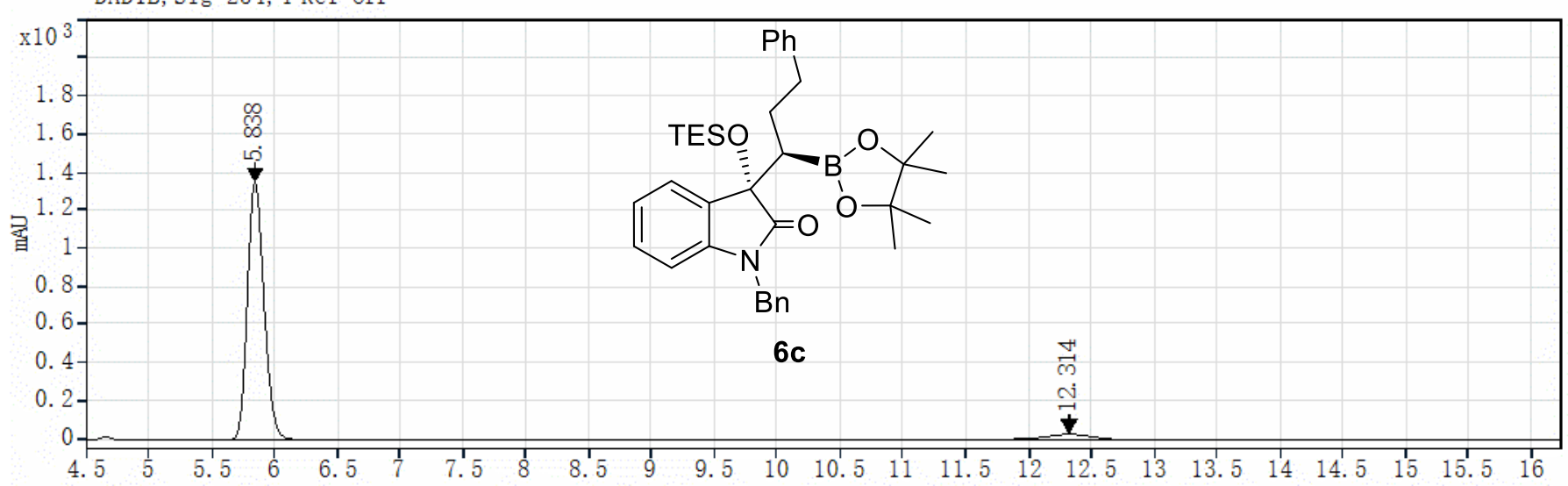

\begin{tabular}{|c|c|c|c|c|c|}
\hline RetTime [min] & Type & Wide [min] & Area[mAu*s] & Height[mAu] & Area $\%$ \\
\hline 5.838 & MM m & 0.14 & 12380.16 & 1347.32 & 95.14 \\
\hline 12.314 & MM m & 0.38 & 632.13 & 25.92 & 4.86 \\
\hline
\end{tabular}


DAD1B, Sig=254, 4 Ref=off

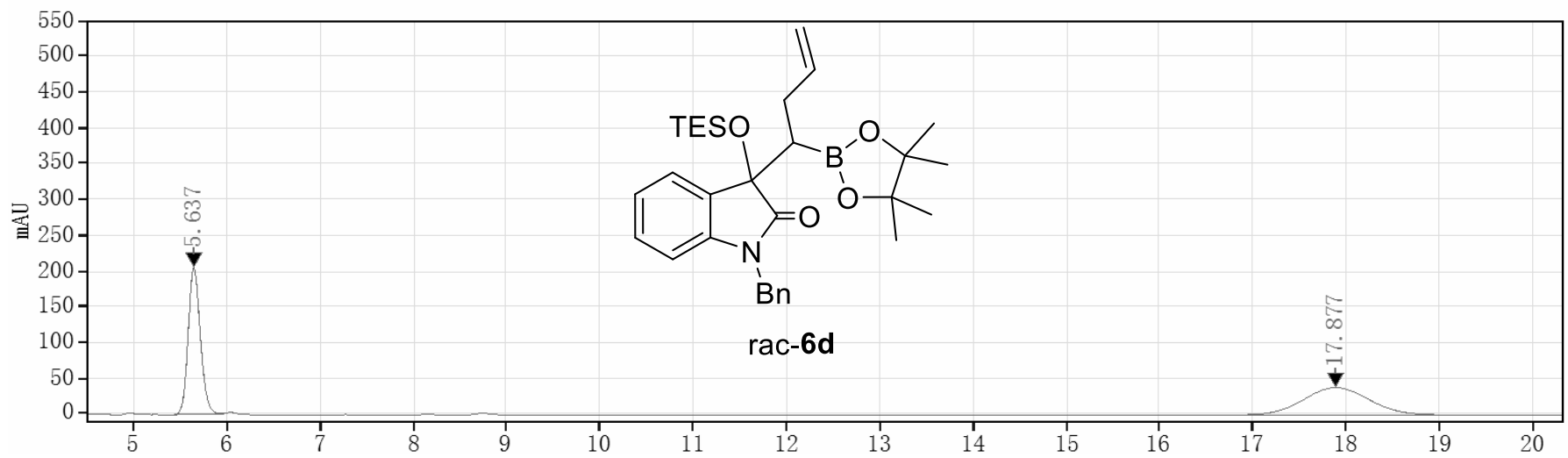

\begin{tabular}{ccccc}
\hline RetTime [min] & Type & Wide [min] & Area[mAu*s & Height[mAu] \\
\hline 5.637 & MM m & 0.14 & 1852.08 & 204.78 \\
17.877 & MM m & 0.71 & 1838.49 & 37.85 \\
\hline
\end{tabular}

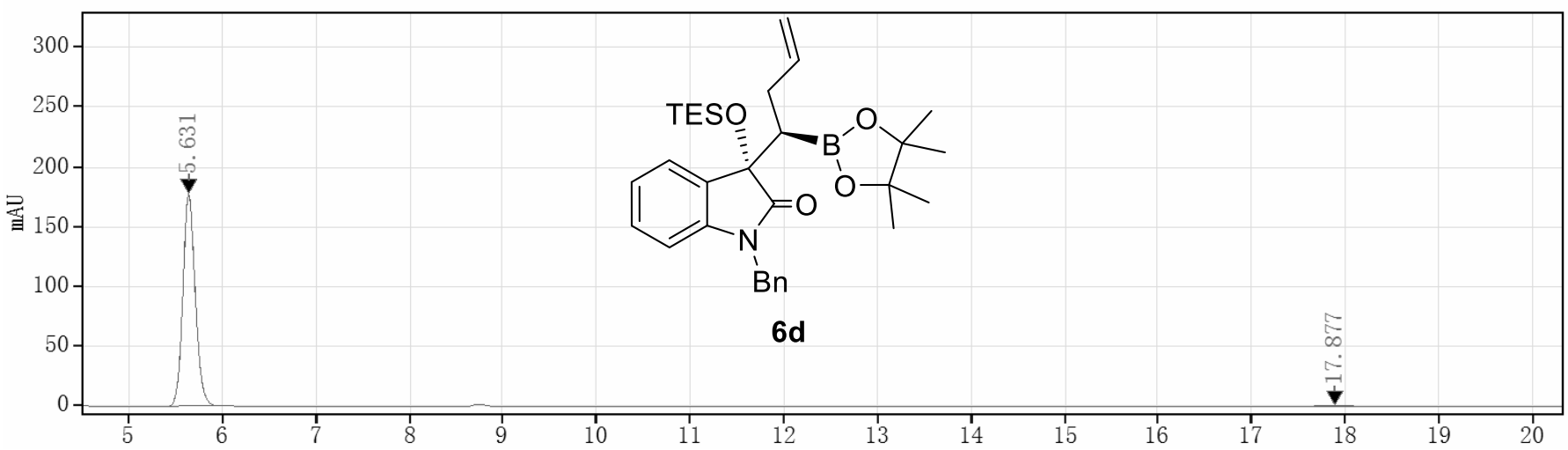

\begin{tabular}{ccccc}
\hline RetTime [min] & Type & Wide [min] & Area[mAu*s] & Height[mAu] \\
\hline 5.631 & MM m & 0.14 & 1623.69 & 177.06 \\
17.877 & MM m & 0.26 & 6.65 & 0.30 \\
\hline
\end{tabular}


DAD1B, Sig=254, 4 Ref=off

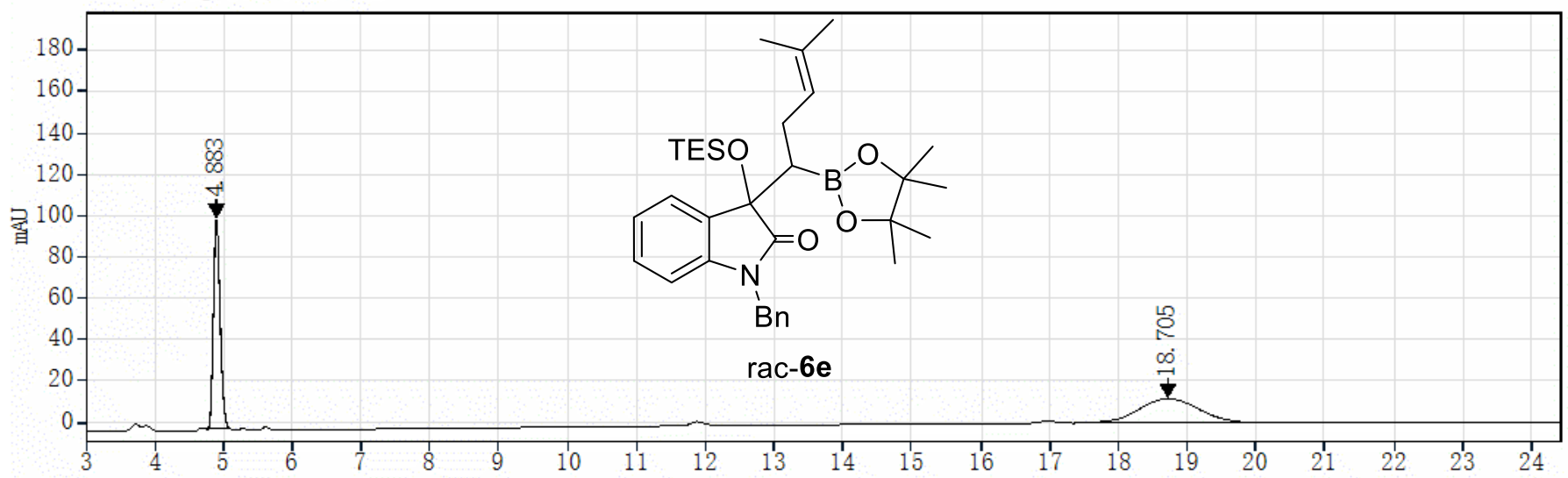

\begin{tabular}{ccccc}
\hline RetTime [min] & Type & Wide [min] & Area[mAu*s & Height[mAu] \\
\hline 4.883 & MM m & 0.11 & 690.95 & 101.48 \\
18.705 & MM m & 0.70 & 677.99 & 11.41 \\
\hline
\end{tabular}

DAD1B, Sig=254, 4 Ref=off

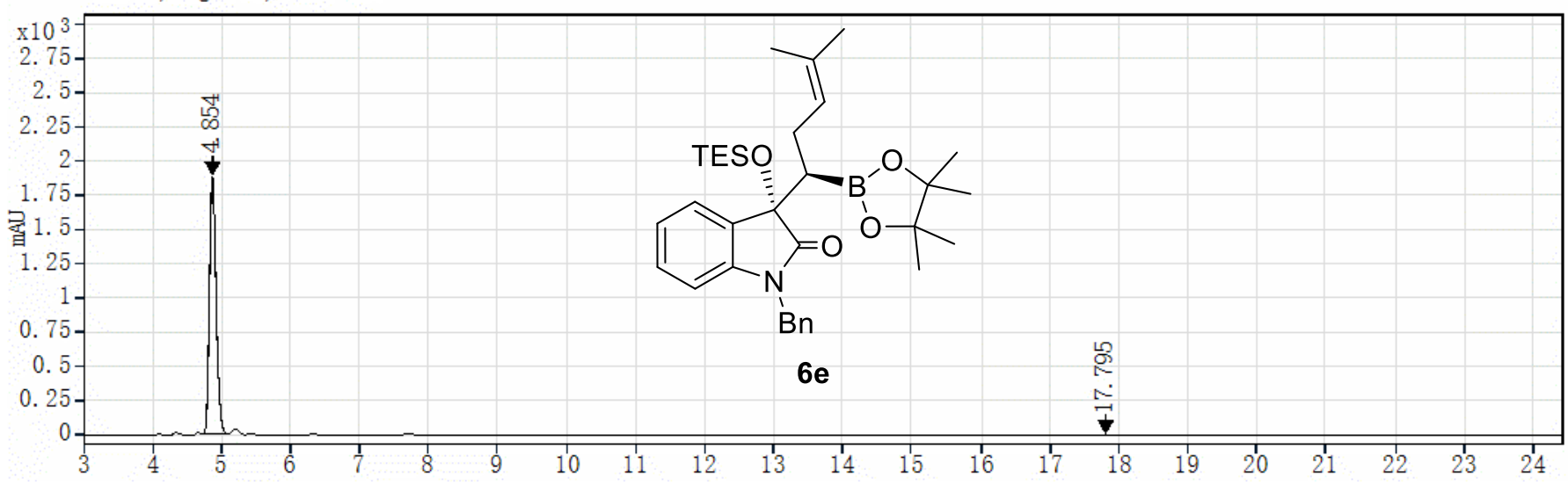

\begin{tabular}{ccccc}
\hline RetTime [min] & Type & Wide [min] & Area[mAu*s] & Height[mAu] \\
\hline 4.854 & MM m & 0.10 & 12624.69 & 1888.47 \\
17.795 & MM m & 0.29 & 60.74 & 2.52 \\
\hline
\end{tabular}


DAD1B, Sig=254, 4 Ref $=$ of $f$

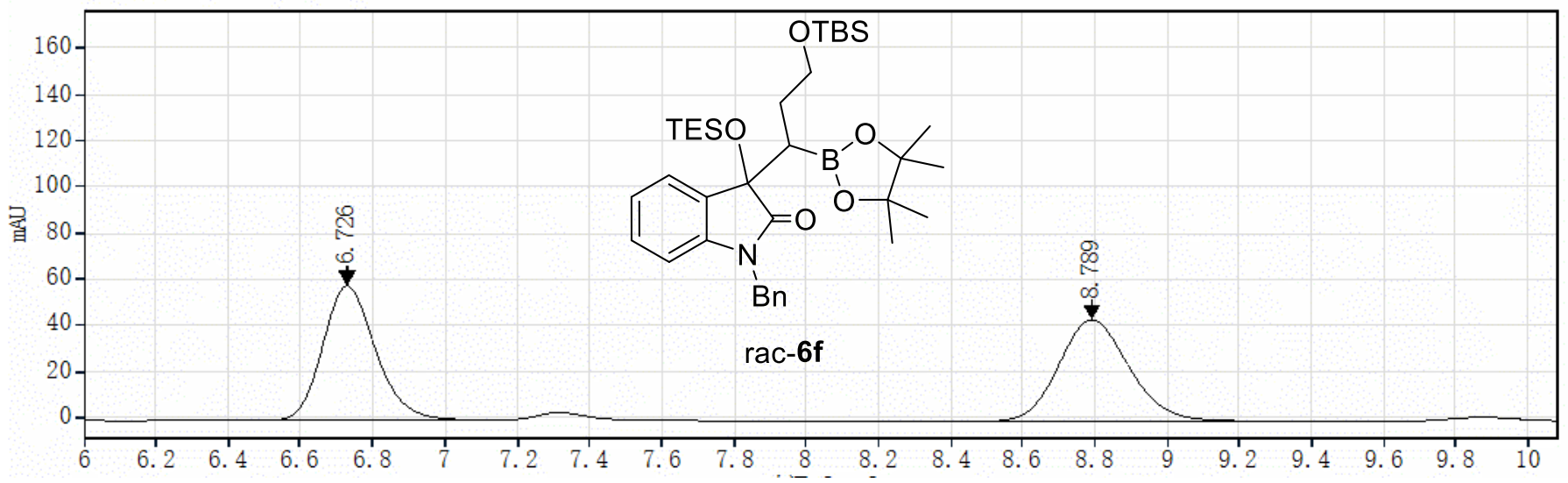

\begin{tabular}{ccccc}
\hline RetTime [min] & Type & Wide [min] & Area[mAu*s] & Height[mAu] \\
\hline 6.726 & MM m & 0.16 & 592.27 & 58.16 \\
8.789 & MM m & 0.21 & 591.45 & 43.90 \\
\hline
\end{tabular}

DAD1B, Sig=254, 4 Ref=off

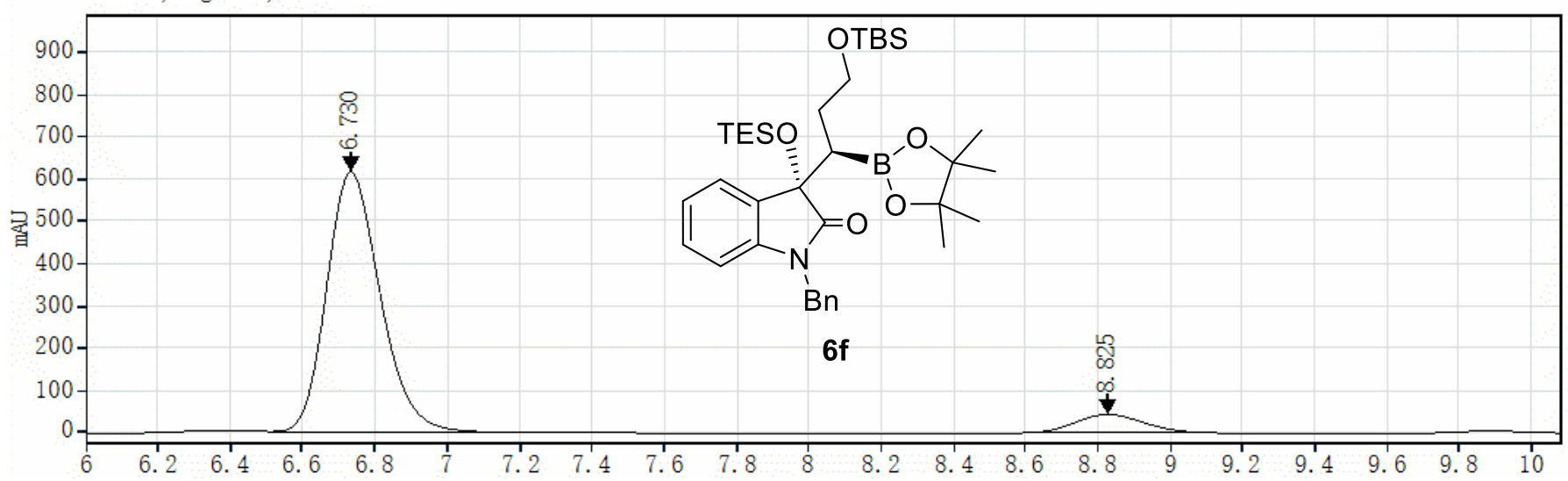

\begin{tabular}{ccccc}
\hline RetTime [min] & Type & Wide [min] & Area[mAu*s] & Height[mAu] \\
\hline 6.730 & MM m & 0.16 & 6324.85 & 616.51 \\
8.825 & MM m & 0.20 & 507.65 & 41.37 \\
\hline
\end{tabular}


DAD1B, Sig=254, 4 Ref=off

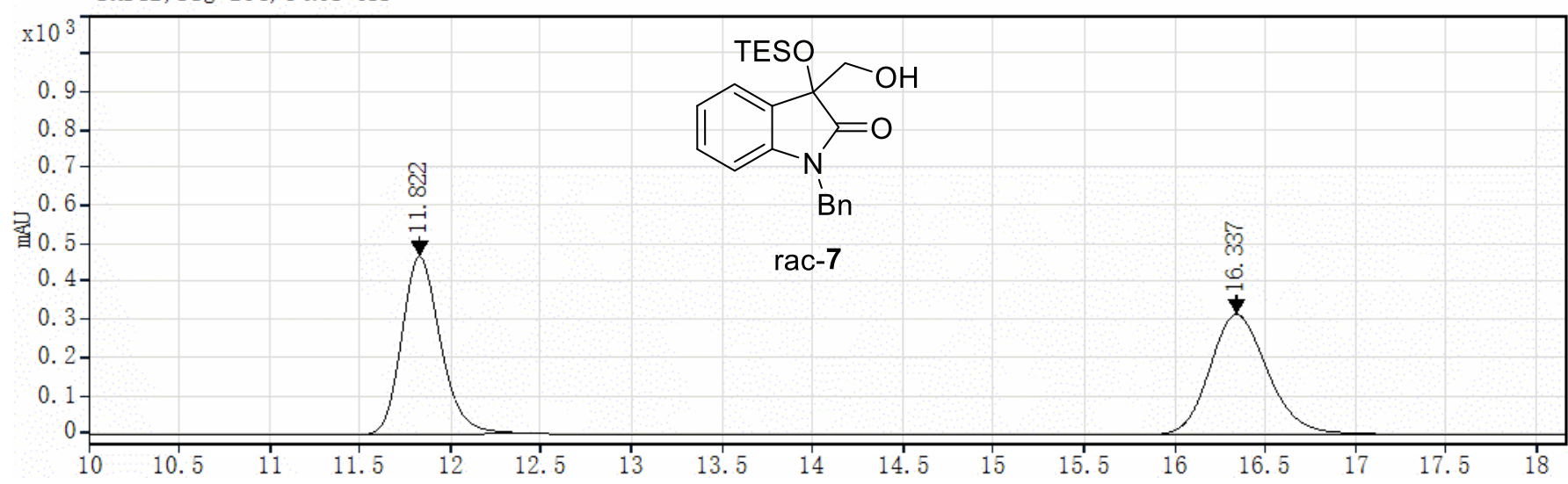

\begin{tabular}{ccccc}
\hline RetTime [min] & Type & Wide [min] & Area[mAu*s] & Height[mAu] \\
\hline 11.822 & MM m & 0.23 & 6978.39 & 468.10 \\
16.337 & MM m & 0.34 & 6975.48 & 315.38 \\
\hline
\end{tabular}

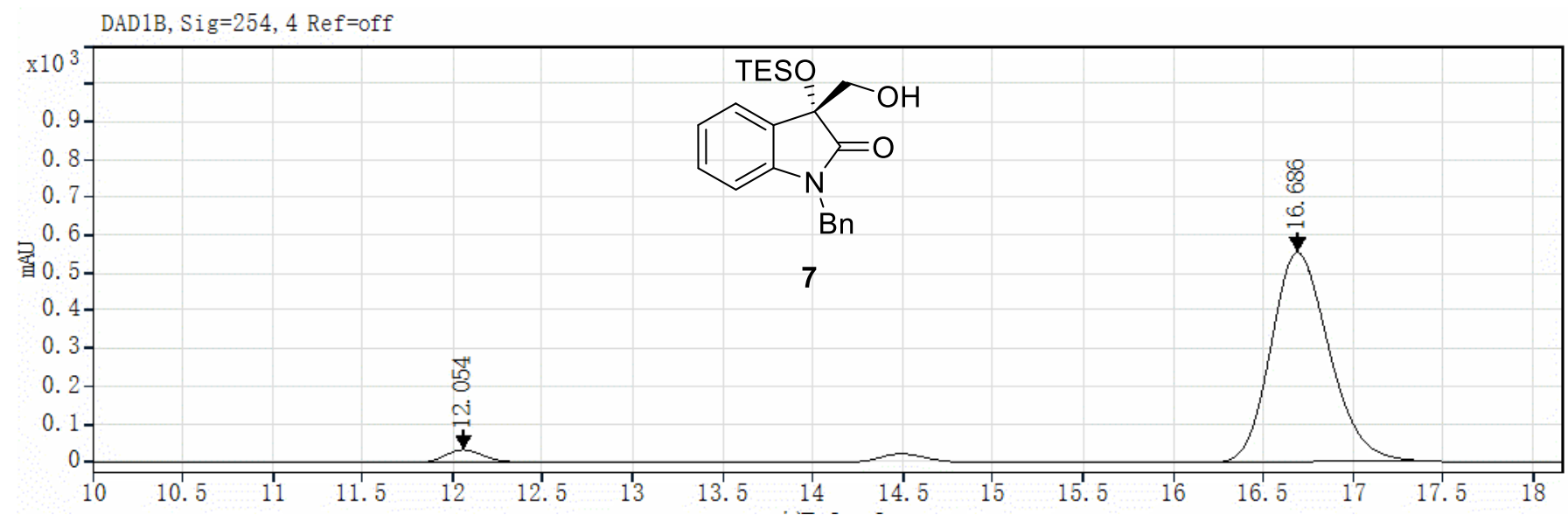

\begin{tabular}{|c|c|c|c|c|c|}
\hline RetTime [min] & Type & Wide [min] & Area[mAu*s] & Height[mAu] & Area $\%$ \\
\hline 12.054 & MM m & 0.22 & 459.98 & 32.70 & 3.58 \\
\hline 16.686 & $\mathrm{MM} \mathrm{m}$ & 0.35 & 12404.89 & 554.24 & 96.42 \\
\hline
\end{tabular}


DAD1B, Sig=254, 4 Ref=off

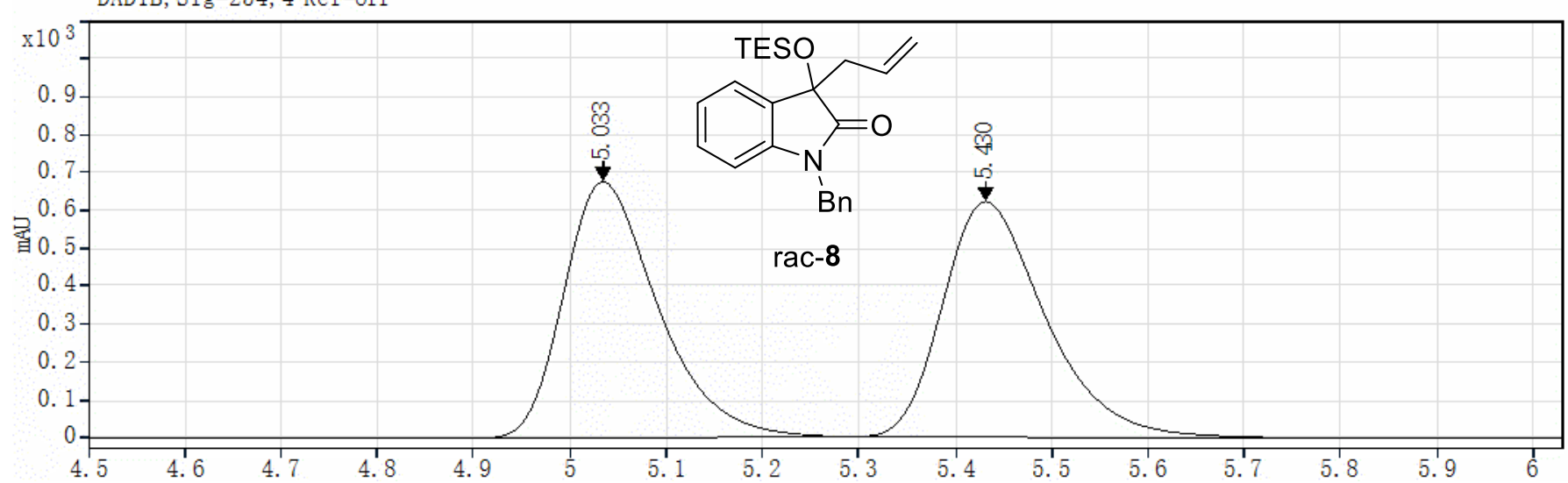

\begin{tabular}{ccccc}
\hline RetTime [min] & Type & Wide [min] & Area[mAu*s] & Height[mAu] \\
\hline 5.033 & MM m & 0.10 & 4606.26 & 676.16 \\
5.430 & MM m & 0.11 & 4621.41 & 621.67 \\
\hline
\end{tabular}

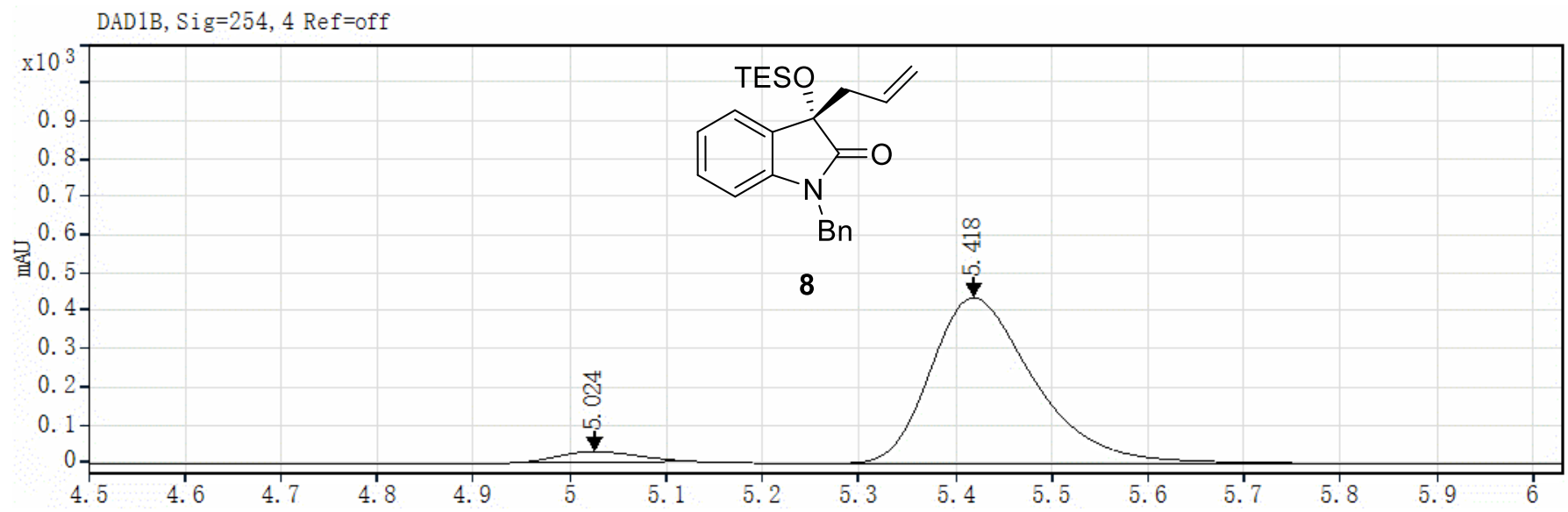

\begin{tabular}{cccccc}
\hline RetTime $[\mathrm{min}]$ & Type & Wide $[\mathrm{min}]$ & Area[mAu*s] & Height[mAu] & Area\% \\
\hline 5.024 & MM m & 0.09 & 153.47 & 27.20 & 4.50 \\
5.418 & MM m & 0.11 & 3254.85 & 436.28 & 95.50 \\
\hline
\end{tabular}




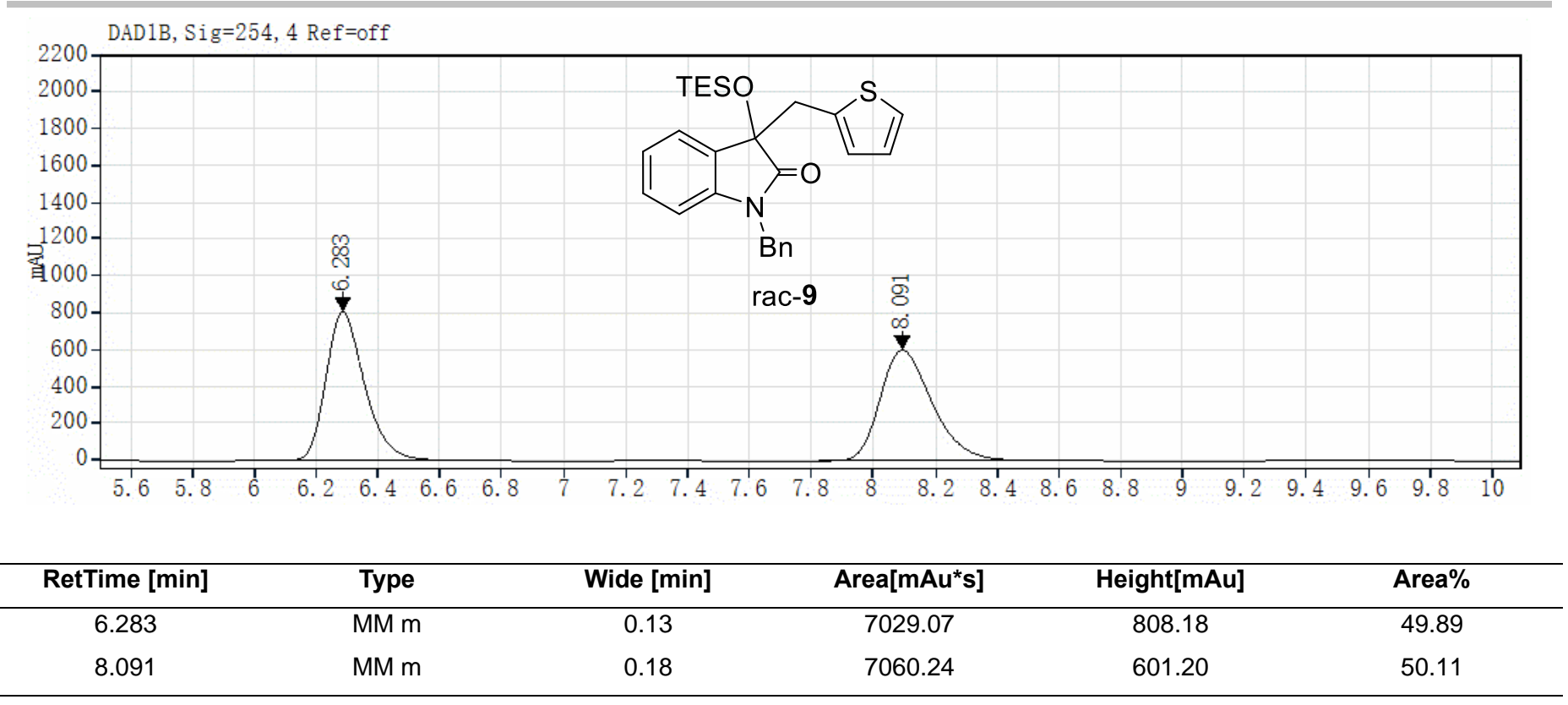

DAD1B, Sig=254, 4 Ref $=$ of $f$

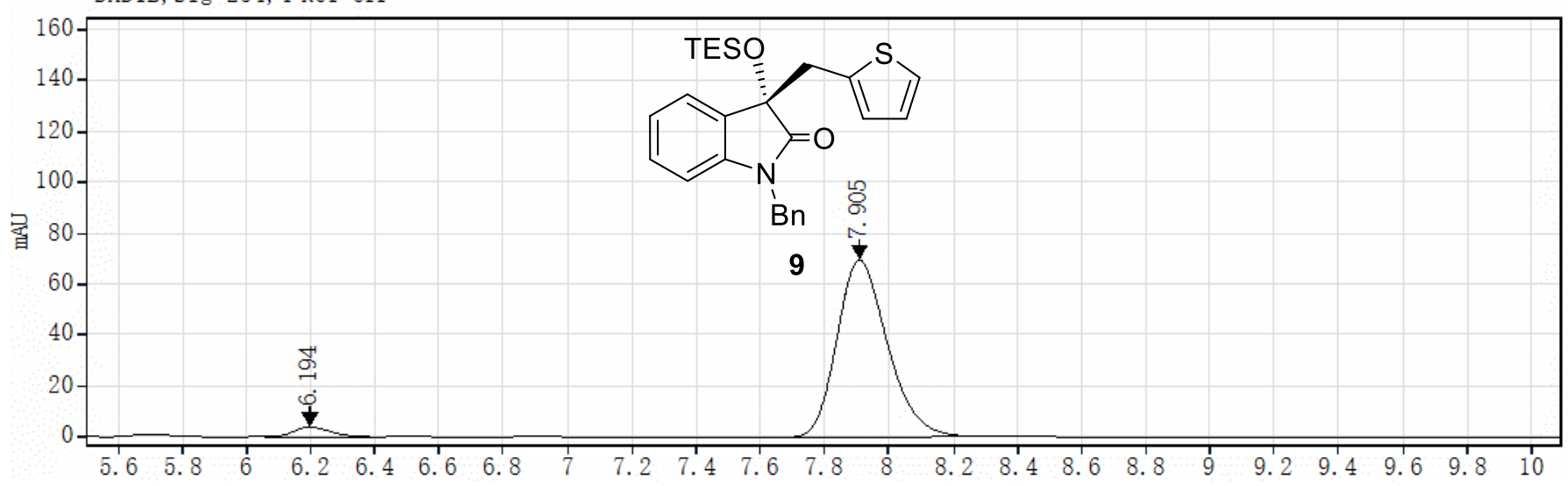

\begin{tabular}{ccccc}
\hline RetTime [min] & Type & Wide [min] & Area[mAu*s] & Height[mAu] \\
\hline 6.194 & MM m & 0.12 & 29.92 & 3.86 \\
7.905 & MM m & 0.17 & 762.12 & 69.73 \\
\hline
\end{tabular}


DAD1B, Sig=254, 4 Ref=off

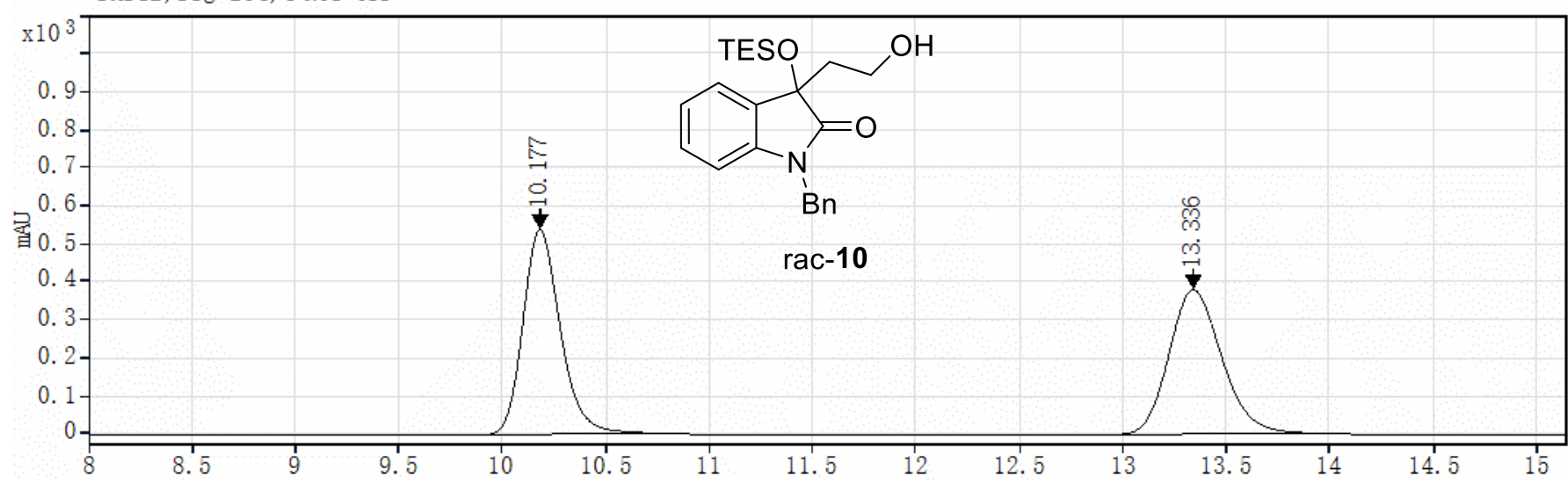

\begin{tabular}{ccccc}
\hline RetTime [min] & Type & Wide [min] & Area[mAu*s] & Height[mAu] \\
\hline 10.177 & MM m & 0.19 & 6727.85 & 538.48 \\
13.336 & MM m & 0.27 & 6730.33 & 380.20 \\
\hline
\end{tabular}

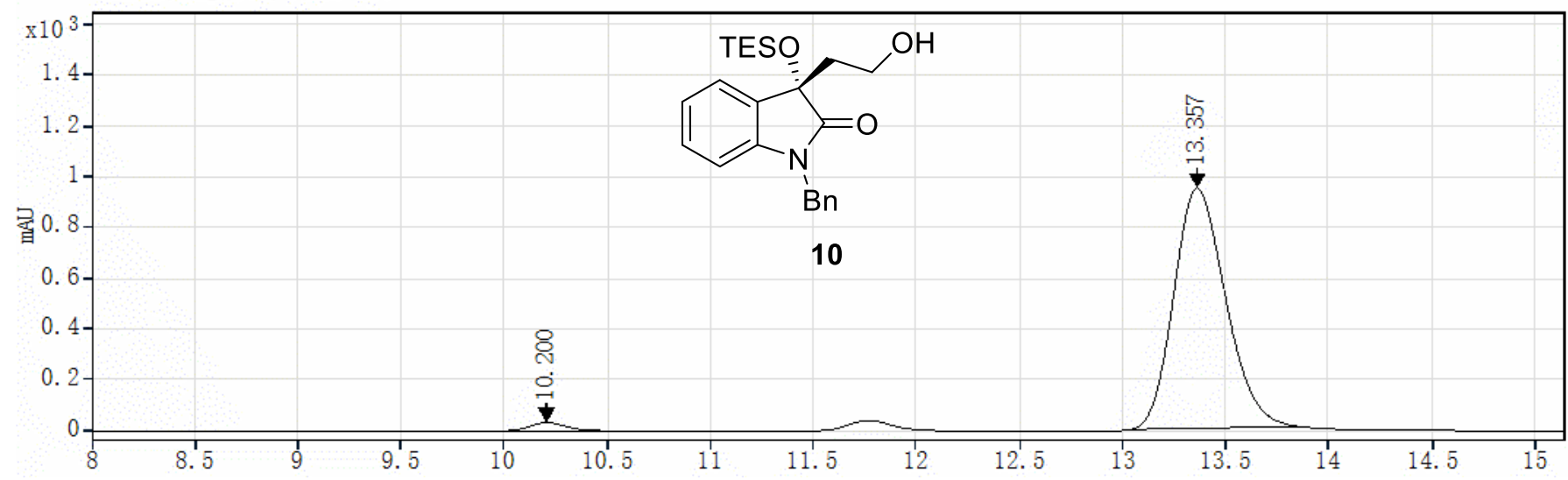

\begin{tabular}{ccccc}
\hline RetTime [min] & Type & Wide [min] & Area[mAu*s] & Height[mAu] \\
\hline 10.200 & MM m & 0.19 & 507.57 & 32.16 \\
13.357 & MM m & 0.27 & 16467.01 & 948.37 \\
\hline
\end{tabular}



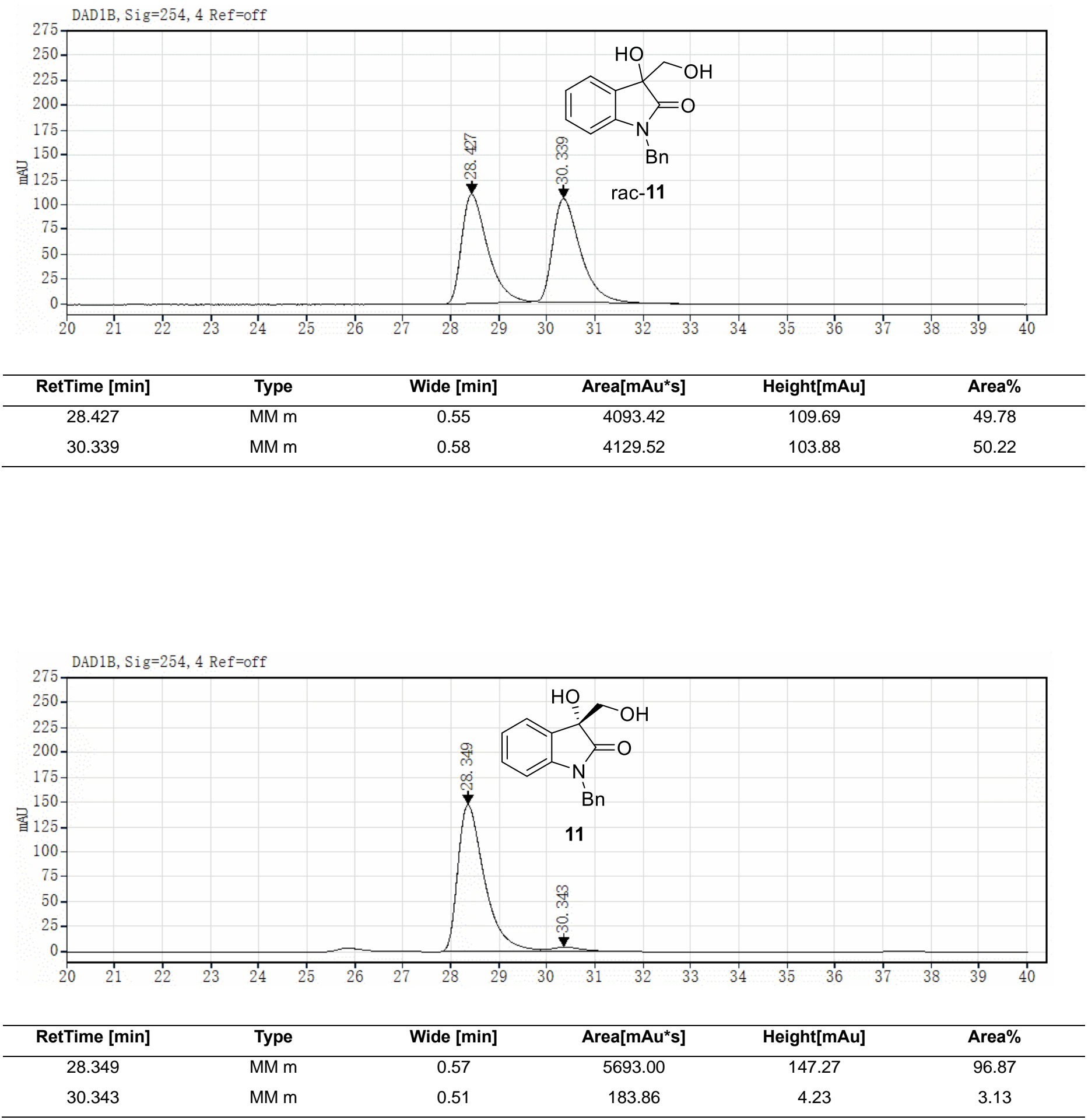
DAD1B, Sig=254, 4 Ref=off

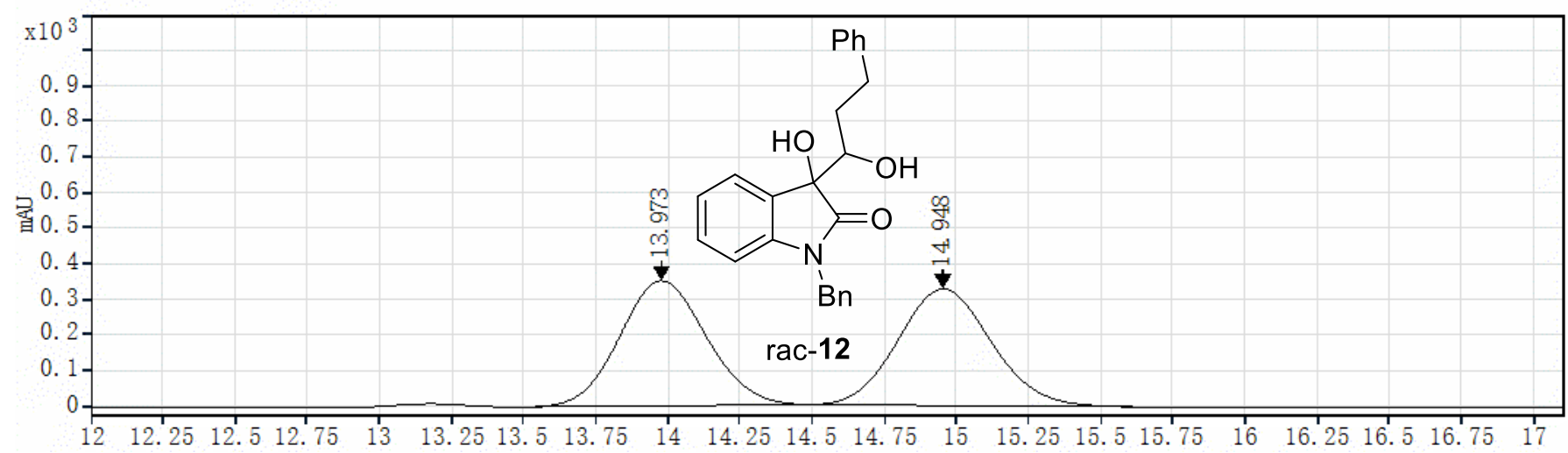

\begin{tabular}{ccccc}
\hline RetTime [min] & Type & Wide [min] & Area[mAu*s] & Height[mAu] \\
\hline 13.973 & MM m & 0.33 & 7344.72 & 351.78 \\
14.948 & MM m & 0.35 & 7386.26 & 327.80 \\
\hline
\end{tabular}

DAD1B, Sig=254, 4 Ref=off

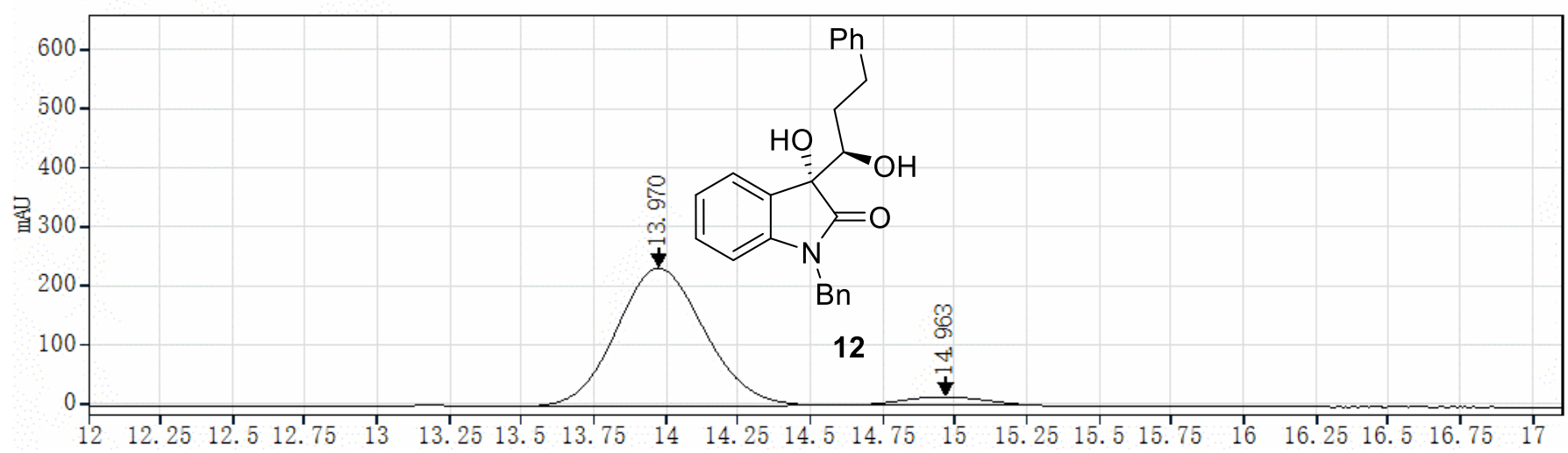

\begin{tabular}{|c|c|c|c|c|c|}
\hline RetTime [min] & Type & Wide [min] & Area[mAu*s] & Height[mAu] & Area\% \\
\hline 13.970 & $\mathrm{MM} \mathrm{m}$ & 0.33 & 4995.37 & 233.94 & 95.99 \\
\hline 14.963 & MM m & 0.23 & 208.14 & 13.07 & 4.01 \\
\hline
\end{tabular}




\section{Crystallographic Data}

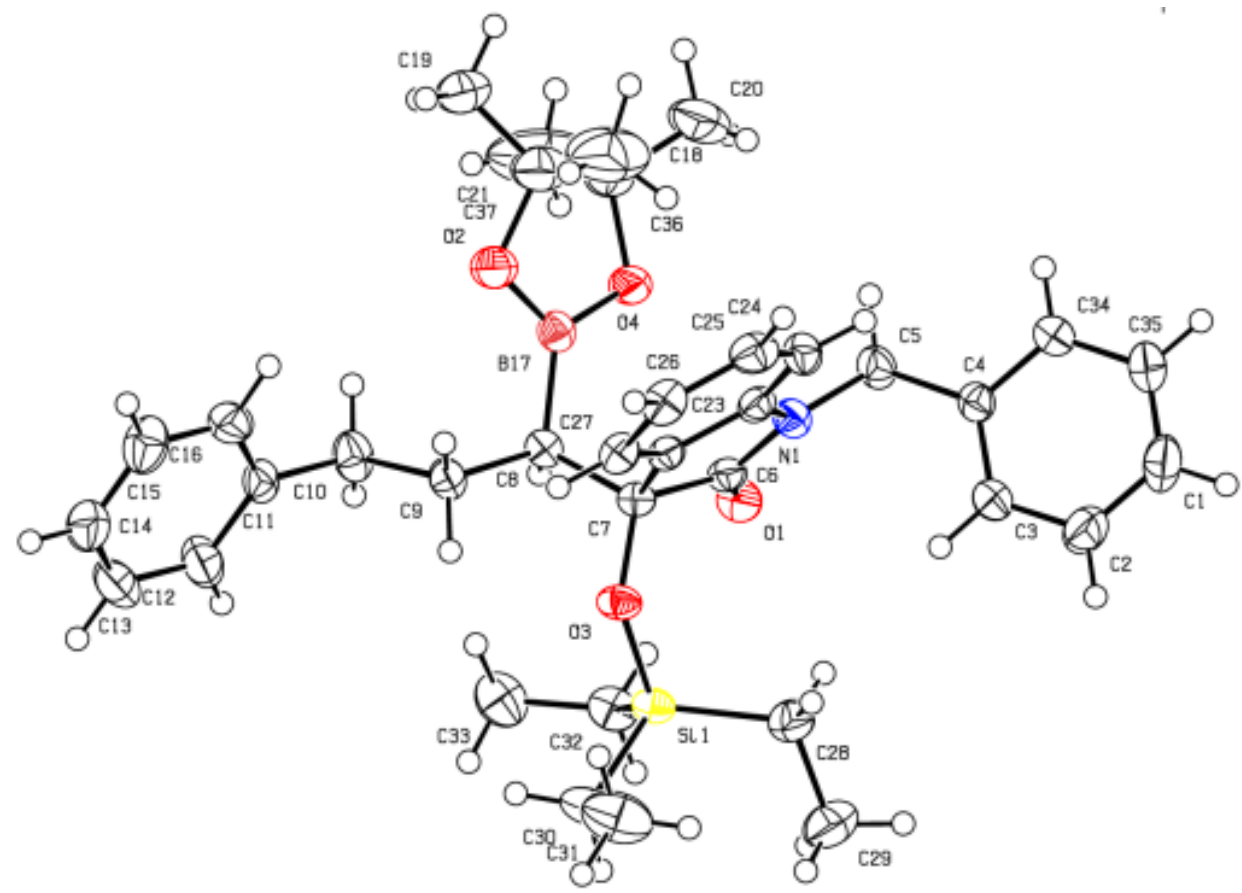

The X-ray Single-Crystal Diffraction Analysis of 6c (CCDC 2091724)

The configuration of the crystal $\mathbf{6 c}$ was first recrystallized and confirmed by chiral HPLC analysis before X-ray crystallography analysis, and the configuration of the crystal is the same compared with the major enantiomer of $6 \mathrm{c}(99 \% \mathrm{ee})$.

The crystals of $6 \mathrm{c}$ were obtained from a solution of dichloromethane and petroleum ether upon slow volatilization. The structure of a derivative of product $6 \mathbf{c}\left(\mathrm{C}_{36} \mathrm{H}_{48} \mathrm{BNO}_{4} \mathrm{Si}\right)$ was determined by X-ray diffraction. The X-ray intensity data were measured at $168.0 \mathrm{~K}$, on a Bruker APEX-II CCD diffractometer with helios $m x$ multilayer monochrmator Cu Ka radiation $(\lambda=1.54178 \AA$ ). The $X$-ray data have been deposited at the Cambridge Crystallographic Data Center (CCDC 2091724). The level set for thermal ellipsoids of all atoms is 50\%.

Table S1. Crystal data and structure refinement for 6c.

Empirical formula

Formula weight

Temperature/K

Crystal system

Space group

$a / \AA$

$\mathrm{b} / \AA$

$\mathrm{c} / \AA$

$\alpha /^{\circ}$

$\beta /^{\circ}$

$\mathrm{\gamma} /{ }^{\circ}$

Volume/Å3
$\mathrm{C}_{36} \mathrm{H}_{48} \mathrm{BNO}_{4} \mathrm{Si}$

597.65

168.0

orthorhombic

$\mathrm{P} 2{ }_{1} 2_{1} 2_{1}$

11.2197(3)

13.0698(3)

$23.3548(6)$

90

90

90

3424.73(15) 
pcalcg $/ \mathrm{cm} 3$

$\mu / \mathrm{mm}^{-1}$

$F(000)$

Crystal size/mm3

Radiation

$2 \Theta$ range for data collection $/{ }^{\circ}$

Index ranges

Reflections collected

Independent reflections

Data/restraints/parameters

Goodness-of-fit on F2

Final $R$ indexes $[\mid>=2 \sigma(I)]$

Final $\mathrm{R}$ indexes [all data]

Largest diff. peak/hole / e Å-3

Flack parameter
1.159

0.896

1288.0

$0.31 \times 0.23 \times 0.15$

$\operatorname{CuKa}(\lambda=1.54178)$

7.57 to 133.374

$-13 \leq h \leq 13,-15 \leq k \leq 15,-27 \leq \mathrm{I} \leq 26$

44881

$6068\left[R_{\text {int }}=0.0538, R_{\text {sigma }}=0.0332\right]$

$6068 / 7 / 395$

1.081

$R 1=0.0528, w R 2=0.1492$

$R 1=0.0564, w R 2=0.1521$

$0.72 /-0.34$

$0.034(10)$

These data can be obtained free of charge from The Cambridge Crystallographic Data Centre via www.ccdc.cam.ac.uk/data request/cif. 